\title{
Perspektiven von Arbeit in der Chemischen Industrie
}

Prozessorganisation und Arbeitsgestaltung

am Beispiel eines Reorganisationsprojekts

\author{
Dissertation \\ zur Erlangung des sozialwissenschaftlichen Doktorgrades der \\ Sozialwissenschaftlichen Fakultät \\ der Georg-August-Universität Göttingen \\ vorgelegt von \\ Kendra Briken \\ aus Alfeld/ Leine \\ Göttingen 2004
}


1. Gutachter: Prof. Dr. Michael Schumann

2. Gutachter: Prof. Dr. Martin Baethge

Tag der mündlichen Prüfung: 16.09.2004 
1 MERKMALE UND ARBEITSFOLGEN DER PROZESSORGANISATION 6

$\begin{array}{llr}1.1 & \text { Veränderungen von Betriebs- und Arbeitsorganisation } & 6\end{array}$

$\begin{array}{lll}1.2 & \text { Transformationstrends industrieller Arbeit im Rahmen der Prozessorganisation } & 8\end{array}$

\section{INNOVATIONS- UND PRODUKTIONSMODELL DER CHEMISCHEN} INDUSTRIE

$\begin{array}{lll}2.1 & \text { Eckdaten zur ökonomischen Entwicklung } & 19\end{array}$

$2.2 \quad$ Technisierungsverläufe der Chemieproduktion 25

2.2.1 Produktion im Großküchenstil 25

2.2.2 Mit der Verfahrenstechnik zur Massenproduktion 26

$\begin{array}{lll}\text { 2.2.3 Automatisierung und Digitalisierung } & 27\end{array}$

2.3 Die Organisation von Innovation 28

2.3.1 Chemie als neue Wissenschaft - Chemische Industrie als neue Industrie 29

2.3.2 Forschungsergebnisse und industrielle Verwertbarkeit 31

2.3.3 Wissenstransfer zwischen Universität und Industrie 32

2.3.4 Zunehmende Autonomie der Industrieforschung 33

2.3.5 Wissenschaftler in der Produktion 36

$\begin{array}{lll}\text { 2.3.6 Zwischenresümee } & 37\end{array}$

2.4 Ausdifferenzierung der Berufe in der Chemischen Industrie 38

2.4.1 Organisatorische und personelle Ausdifferenzierung im akademischen Bereich 38

2.4.2 Diversifizierung und Spezialisierung in der Instandhaltung 41

2.5 Produktionsarbeit in der Chemischen Industrie 43

$\begin{array}{lll}\text { 2.5.1 Anforderungsprofile und technischer Wandel } & 43\end{array}$

2.5.2 Ausbildung und Kompetenzprofile 48

2.6 Hierarchische Strukturen und Sozialgefüge $\quad 57$

2.6.1 Interne Organisationsstruktur und Hierarchieebenen $\quad 57$

$\begin{array}{lll}2.6 .2 & \text { Kooperations- und Statusmodell }\end{array}$

2.7 Zwischenresümee

3 ENTWICKLUNG DES INNOVATIONS- UND PRODUKTIONSMODELLS $\begin{array}{ll}\text { IN DEN 1990ER JAHREN } & 73\end{array}$

$\begin{array}{lll}3.1 & \text { Von der verbund- zur portfolioorientierten Unternehmensorganisation }\end{array}$

3.2 Das Innovationsmodell der Chemischen Industrie vor neuen technologischen Herausforderungen 


\section{INNOVATIVE ARBEITSGESTALTUNG IN DER BETRIEBLICHEN} PRAXIS

4.1 Das Projekt „Prozessorientierte Fertigung im Team“: Genese und Zielsetzungen

4.3 Arbeitsbedingungen und Arbeitswahrnehmung im Kontext innovativer Arbeitspolitik

4.3.2 Gestaltungsziel Aufgaben- und Funktionsintegration

4.3.3 Gestaltungsziel Gruppengetragene Selbstorganisation

4.3.4 Gestaltungsziel Meister als Unterstützer der Gruppe

4.3.5 Gestaltungsziel Einbindung der Beschäftigten in die betriebliche Organisation

4.3.6 Projektgestaltung im Urteil der Beschäftigten

4.4 Markt-, Produkt- und Innovationssituation in ihrer Bedeutung für innovative Arbeitsgestaltung

4.4.1 Neue Formen der Arbeitsorganisation bei gering ausgeprägter Innovationsaffinität 128

4.4.2 Neue Formen der Arbeitsorganisation bei starker Innovationsaffinität 135

4.5 Neustrukturierung von Hierarchie und Kompetenzen 142

4.5.1 Betriebsmeister: Vom Personalvorgesetzten zum Fachkoordinator 142

4.5.2 Vom Schichtführer zum Schichtmeister und Coach 152

$\begin{array}{llr}\text { 4.6 } & \text { Fazit: Nicht nur die Chemie muss stimmen... } & 160\end{array}$

$5 \quad$ SCHLUSSBEMERKUNGEN 167

6 LITERATURVERZEICHNIS 


\section{Einleitung}

Diese Arbeit analysiert den Umsetzungsprozess eines Reorganisationsprojektes in einem Großunternehmen der Chemischen Industrie, das von Unternehmen und Betriebsrat einvernehmlich als innovative Form der Gruppenarbeit konzeptualisiert und beschlossen wurde. Kompetenz, Erfahrung und Kreativität der Beschäftigten sollten durch diese institutionalisierte Formen der Selbstorganisation gezielt gefördert, zeitliche und inhaltliche Freiräume für die Beteiligung an Innovationsaktivitäten gestärkt und insgesamt kommunikative und vernetzte Arbeitsformen gestützt werden. Explizit geht es darum, das Wissen und die produktiven Potentiale der Beschäftigten als Ressource und eigenständigen Beitrag in den Innovationsprozess einzubeziehen. Für die Fabrikwelt der Chemiearbeit ist damit ein sehr weitgehendes Reorganisationsprojekt lebendiger Arbeit umrissen, das zugleich in einen umfassenden technologischen, betriebsorganisatorischen und ökonomischen Umbau der Chemischen Industrie eingebettet ist.

Zentrales Movens dieses Umbaus ist, bringt man die sozialwissenschaftlichen Debatten der letzten Dekade auf den Punkt, die oft zitierte „Innovationsschwäche“ der deutschen Chemischen Industrie. Zwar verfügen die Unternehmen in der Regel über eine im internationalen Vergleich herausragende Ausstattung an qualifizierten Beschäftigten auf allen hierarchischen Ebenen, dennoch schneiden sie im globalen Innovationswettbewerb zunehmend schlechter ab (ZEW 2003). Vor diesem Hintergrund gerät die bürokratischzentrale Steuerung und Kontrolle der unterschiedlichen Organisationseinheiten, jahrzehntelang als „one best way“ der Unternehmenskoordination vertreten und praktiziert, unter erheblichen Veränderungsdruck. Der Output von Produkt- und Prozessinnovation ist unter den Prämissen der traditionellen, zentralistischen Entwicklungsorganisationen der Unternehmen zu gering. Die etablierten arbeitsteiligen Organisationsstrukturen gelten als kontraproduktiv bei der Hervorbringung wissenschaftlich-technischer Innovationen.

Seit Mitte der 1990er Jahre steht also nicht nur die Reorganisation der Arbeit, sondern auch die des Innovationsprozesses auf der Agenda der chemischen Großunternehmen, womit ein für den weiteren Argumentationsgang dieser Untersuchung entscheidender Sachverhalt angesprochen ist. Denn in dieser Konstellation verändern sich nicht nur die Formen der Kooperation von Unternehmen untereinander sowie mit externen Forschungseinrichtungen und der interorganisatorische Kontext der Erzeugung von Innovationen wird modifiziert. Darüber hinaus wird auch der binnenorganisatorische Zusammenhang von Innovation und Arbeit transformiert (Scharping 2000; Kurz 2002). Einerseits geht es dabei um die Reorganisation der Forschungs- und Entwicklungsabteilungen, in deren Folge insbesondere der Produktentstehungsprozess optimiert und das kreative und selbsttätige Potential der Beschäftigten stärker zum Tragen kommen soll. Gleichzeitig steht andererseits auch die ehemals strikte Trennung von Produktions- und Innovationsfunktionen zur Disposition. Ziel ist es, Produktion und Produkt- sowie Prozessentwicklung enger miteinander zu verkoppeln (D’Alessio et al. 2000). Die Reorganisation der gesamten Wertschöpfungskette von der Forschung über die Entwicklung bis in die Produktion hinein steht im Fokus der Restrukturierungsansätze der Unternehmen. Zum neuen „geschäftspolitischen Credo“ (Baethge et al. 1998) wird die gleichzeitige Optimierung von Markt- und Kundenbezug, Produktqualität, schnellerer Innovation und Kostensenkung. Aus soziologischer Perspektive ist damit ein organisationaler Umbruch signalisiert, 
der idealtypisch als Wandel von einer funktions-berufsorientierten zu einer prozessorientierten Betriebs- und Arbeitsorganisation beschrieben werden kann.

In dieser Untersuchung soll auf Basis empirischer Einzelfallanalysen eine differenzierte Vorstellung darüber entwickelt werden, worin die neue Qualität der Chemiearbeit, hier verstanden als Produktionsarbeit, unter den Prämissen einer auf innovativer Arbeitspolitik basierenden Prozessorganisation besteht. Es wird analysiert, in welchen betrieblichen Konstellationen der neue Zugriff auf Arbeit wirklich greift, wo er zum Missgriff wird, welche neuen Widersprüche und sozialen Konfliktstoffe er schafft.

Der erste Teil der Leitthese lautet, dass die Veränderungen im Bereich der Betriebs- und Innovationsorganisation die arbeitsorganisatorische Entwicklungsdynamik und mit ihr innovative Arbeitsformen fördern bzw. hemmen können. Anders formuliert: Die Durchsetzungsbedingungen und Durchsetzungsmöglichkeiten innovativer Arbeitspolitik hängen entscheidend davon ab, ob und wie weit sie mit dem Umbau von Betriebs- und Innovationsorganisation konvergieren.

Eine wesentliche Voraussetzung für die Nutzung der kreativen Potentiale menschlichen Arbeitshandelns im Sinn von Innovationsarbeit ist, so der zweite Teil der These, die Bedeutung, die dem Shop floor für die Dynamik und den Erfolg von Innovationsprozessen vom betrieblichen Management zugewiesen wird. Wird hier ein hohes Potential vermutet, so wird Innovationshandeln, verstanden als Planung, Steuerung und Optimierung, zum inhärenten Bestandteil der Anforderungsprofile auf dem Shop floor. Wird diese eher gering eingeschätzt, bleibt es bei einer spezialistenzentrierten Definition der Innovationsarbeit, und den Beschäftigten wird in der Regel eine Rationalisierungsbeteiligung in Form von Selbstökonomisierung zugewiesen. Wir werden anhand des empirischen Materials zeigen, dass die unternehmerische Absicht zur Einführung selbstorganisierter Gruppenarbeit die Umsetzung in der betrieblichen Praxis zwar beeinflusst, diese aber nicht determiniert. Vielmehr bewegen sich die Betriebe im Rahmen bestimmter Entscheidungs- und Handlungskorridore, die von Produkt- und Marktstrukturen, Organisationskultur, der Verteilung von Ressourcen und zeitlich vorgelagerten Entscheidungen geprägt ist (Ortmann 1995). Um Arbeitsveränderungen vor diesem Hintergrund angemessen erfassen zu können, wird eine Erweiterung der arbeitssoziologischen Analyseperspektive um Dimensionen der Betriebs- und Innovationsentwicklung notwendig.

In Kapitel 1 wird die Prozessorganisation als analytischer Fluchtpunkt bestimmt und die für diese Arbeit wichtigen Begriffe definiert. Dabei werden zunächst grundlegende industriesoziologische Referenzpunkte beleuchtet sowie Transformationstrends industrieller Arbeit aufgezeigt.

In Kapitel 2 wird es dann darum gehen, die Spezifität der Branche im Hinblick auf das Innovations- und Produktionsmodell zu analysieren und die daraus resultierenden Konsequenzen für das Kompetenz-, Kooperations- und Statusmodell zu dechiffrieren. Die Einordnung oder besser Verortung der Chemischen Industrie in ein tayloristisch-fordistisches Konzept der Unternehmens-, Produktions- und Arbeitsorganisation ist im Gegensatz etwa zur Automobil- oder Elektroindustrie selbst für die Blütezeit des Fordismus (bzw. des fordistischen Regimes) nur sehr eingeschränkt möglich und wenig fruchtbar. 
Wenn sich die Chemische Industrie einer Bestimmung entlang des bis in die 1980er Jahre hinein gängigen oder besser gesagt stilbildenden industriellen Produktionsmodells entzieht, dann ergeben sich aus diesem Sachverhalt weitreichende Folgewirkungen für die Analyse der Auflösung und Neuformierung des Innovations- und Produktionsmodells, die seit Anfang der 1990er Jahre auch in der Chemischen Industrie beobachtbar und die in Kapitel 3 zu skizzieren sind.

In Kapitel 4 schließlich wird die These empirisch überprüft. Das im Zentrum der Untersuchung stehende Unternehmen hat einen umfassenden Wandel von einer funktions- und berufsorientierten zu einer prozessorientierten Betriebs- und Arbeitsorganisation vollzogen. Anhand des empirischen Materials wird nachgewiesen, dass die arbeitsorganisatorische Entwicklungsdynamik in der Chemischen Industrie in besonderem Maß von einer kommunikativ-kooperativ geprägten Einbindung der Beschäftigten in die Betriebs- und Innovationsorganisation abhängig ist, und dieser Umbau tiefgreifende Veränderungen für das tradierte Status-, Kompetenz- und Kooperationsmodell impliziert. Darüber hinaus wird über die analytische Ausweitung der arbeitssoziologischen Perspektive um Dimensionen der Betriebs- und Innovationsentwicklung am Beispiel der unterschiedlichen Umsetzungsvarianten gezeigt, dass die Innovationsaffinität der Betriebe eine bislang unterbelichtete Variable für die Tiefe und Reichweite von Arbeitsveränderungen darstellt. 


\section{Merkmale und Arbeitsfolgen der Prozessorganisation}

Obgleich die Formulierung einer schlüssigen Theorie zum Zusammenhang sozioökonomischer Entwicklungen und Unternehmensstrategien aussteht (Schumann 2003), ist davon auszugehen, dass die „säkulare Strukturkrise“ Anfang der 1990er Jahre den bis heute anhaltenden Trend eines tiefgreifenden industriellen Wandels maßgeblich bestimmt. Etablierte Rationalisierungsmodelle erweisen sich als zunehmend dysfunktional bei der Sicherung von Wettbewerbsfähigkeit. Das strategische Zentrum des zu beobachtenden unternehmerischen Rationalisierungszugriffs erweitert sich erheblich. Die Verbindung technologischer und organisatorischer Umgestaltungen mit neuen Strategien der Kapitalverwertung schlägt sich in einer Rationalisierungsdynamik nieder, die vor allem auf veränderten Mustern unternehmerischer Steuerung und Kontrolle basiert. Eine prozessorientierte Betriebs- und Arbeitsorganisation scheint sich als neues Paradigma der Unternehmensgestaltung durchzusetzen (Baethge, Baethge-Kinsky 1998; Schumann 2002).

\subsection{Veränderungen von Betriebs- und Arbeitsorganisation}

Die Betriebsorganisation wandelt sich dabei einmal im Hinblick auf das betriebliche Leistungsprofil. Die stabilen, integrierten Funktionen werden von dynamischen abgelöst, die sich aus einem flexiblen In- und Outsourcing in Richtung auf marktevaluierte Kompetenzkerne ergeben. In den Unternehmen dominiert zunehmend das „Primat der Effizienzsteigerung“ (Springer 1998) und eine stärker dem Shareholder - Value verpflichtete Kapitalverwertungsstrategie (Schumann 1998). Dezentralisierung, Vermarktlichung und diskursive Koordinierung werden zu Schlüsselelementen neuer Rationalisierungsmuster (Sauer, Döhl 1997; Tullius 2003). Der Abbau von Hierarchie und Bürokratie sowie eine verstärkte Nutzung von Selbstorganisation als Steuerungsmechanismus tragen zu einer Auflösung zentralistischer Organisationseinheiten bei. Dezentralisierungsbestrebungen bilden somit eine organisatorische Seite dieser Rationalisierungsstrategie ab und gehören zumindest für die 1990er Jahre zum neuen unternehmerischen Leitbild (Hirsch-Kreinsen 1995). Zentral gebündelte, gleichartige Aufgaben und Zuständigkeiten werden segmentiert und auf neudefinierte organisatorische Subeinheiten verlagert. Ihren Ausdruck finden solche Dezentralisierungsprozesse in einer Divisionalisierung in Unternehmensbereiche, deren Kernkompetenzen entlang von Produkten, Produktgruppen oder Kundengruppen redefiniert werden. Die Muster der betrieblichen Funktionsdifferenzierung geraten in Bewegung, und das bis dahin die Betriebsorganisation prägende Fachabteilungsprinzip weicht multi-funktionalen Unternehmenseinheiten mit weitgehender Eigenverantwortlichkeit. Die interne Organisation der Divisionen folgt dabei unterschiedlichen Steuerungsmodi, in einer eher klassisch-funktionalen Gliederung bleibt es bei einer engen Bindung an die strategische Spitze, zunehmend werden aber auch Unternehmensbereiche in rechtlich selbständige Einheiten umgewandelt.

Diese Veränderungstendenzen der Organisation betrieblicher und überbetrieblicher Innovationsprozesse vermitteln sich auch in einer Dezentralisierung der betrieblichen Innovationskompetenz. Die prozessorientiertere Ausrichtung der Arbeitsorganisation fokussiert auf eine systematischere, schnellere und effektivere Nutzung, Mobilisierung und Integration von Qualifikationen und Kompetenzen aller Beschäftigten. Technische, funktionale 
und betriebliche Grenzen werden verschoben, und Ressourcen und Potentiale der betrieblichen Akteure neu verknüpft und gesteuert. Im Mittelpunkt steht dabei die Integration von Innovations- und Produktivitätspotentialen des gesamten Wertschöpfungsprozesses, d.h. der Einbezug aller an ihm beteiligten Beschäftigtengruppen und ihrer jeweils unterschiedlichen Kompetenz- und Wissensbestände. Auf arbeitsgestalterischer Ebene manifestiert sich diese zunehmende Prozessorientierung insbesondere in der Einführung von Projektteams, die querfunktional besetzt sind und eine neue Qualität der Vermittlung zwischen Hierarchie und Team darstellen, die sich in veränderten Kontroll- und Steuerungsmodi manifestiert.

Die betriebswirtschaftlichen Managementstrategien setzen für diesen anspruchsvollen Ansatz auf Konzepte, die eine Definition von Kernprozessen anhand der Kernkompetenzen der Unternehmung vorsieht. Im Rahmen eines solchen Business Reengineering (Hammer, Champy 1994) etwa werden die betrieblichen Strukturen anhand von Geschäftsprozessen, verstanden als typische betriebliche Abläufe, reorganisiert. Berücksichtigt wird dabei die möglichst schnittstellenfreie Verbindung von Teilprozessen, d.h. einzelner Aktivitäten. Aus soziologischer Perspektive ist damit ein organisationaler Umbruch signalisiert, der idealtypisch als Wandel von einer funktions-berufsorientierten zu einer prozessorientierten Betriebs- und Arbeitsorganisation verstanden werden kann (Baethge, Baethge-Kinsky 1998).

Die Prozessorganisation hat weitreichende Folgen für die Arbeitsstrukturen: Erstens wird das fach- und aufgabenzentrierte Kompetenzmodell der funktionsorientierten Organisation redefiniert und die Spezialisierung entlang berufstypischer Qualifikationen weicht zunehmend einer prozessorientierten Auflockerung berufstypischer Einsatzkonzepte und Aufgabenprofile. Dies bedeutet zweitens, dass das zumeist dem Prinzip des Dienstwegs verpflichtete Kooperationsmodell abgelöst wird von einer direkteren Kommunikation und Kooperation und zwar sowohl in der horizontalen wie auch vertikalen, also hierarchieübergreifenden Richtung. Und schließlich gilt drittens für das Statusmodell, dass Hierarchien abgebaut werden und formale Kompetenzen eine geringere Rolle für die innerbetriebliche Karriere spielen. In dieser „innovationszentrierten Produktion“ wird der Zusammenhang exklusiver Zuordnung von Innovationskompetenz, hierarchisch geprägter Koordination und hochgradiger Statusdifferenzierung aufgeweicht (Baethge, BaethgeKinsky 1998:126f.). Innovationen anzuregen impliziert damit selbst bereits eine wichtige organisatorische Innovation, die u.a. dazu dienen soll, betriebliche Prozesse in kleineren Sequenzen zu optimieren und damit den Innovationsprozess insgesamt effizienter zu gestalten (Kocyba 2000). 


\subsection{Transformationstrends industrieller Arbeit im Rahmen der Prozessorganisati- on}

Arbeitsstrukturen und Beschäftigungsformen stehen unter dem Einfluss eines Wirkgefüges, dessen Koordinaten sich aus den neuen Gestaltungsansätzen für die Organisation von Arbeit, Betrieb und Innovation sowie traditionellen Kostensenkungsmaßnahmen und Personalabbau zusammensetzen. Für die Entwicklung von Industriearbeit auf dem Shop floor schlägt sich dies in einer Umsetzung arbeitsgestalterischer Konzepte und der Erprobung neuer Formen der Arbeitsorganisation nieder, die sich mehr oder weniger eindeutig von den tayloristischen Prämissen unterscheiden. Unter dem vereinheitlichenden Label Gruppenarbeit haben diese ganz unterschiedliche Implikationen für die Nutzung von Arbeitskraft. $^{1}$ Dies wird in der sozialwissenschaftlichen Literatur ${ }^{2}$ einmal auf der Konzeptebene von Gestaltungsansätzen nachgewiesen, die maßgeblich die Tiefe und Reichweite von Arbeitsveränderungen bestimmen: Zu differenzieren ist zwischen einer „Modernisierung“ der tayloristisch geprägten Arbeitsorganisation einerseits und einer eine Abkehr und Aufhebung von tradierten arbeitsorganisatorischen und arbeitsgestalterischen Prämissen andererseits. Anhand empirischen Materials aus der Evaluation unterschiedlicher Projekte zur Einführung von Gruppenarbeit in der Automobil- und Elektroindustrie entwickelte das SOFI in den 1990er Jahren den „modifizierten Taylorismus“ und „selbstorganisierte funktionsintegrierte Gruppenarbeit“ als konzeptuelle Idealtypen, denen sich die in der Empirie nachweisbaren Mischformen zuordnen lassen. ${ }^{3}$

In Konzepten, die in ihrer Umsetzung auf modifiziertem Taylorismus basieren, ändert sich wenig an den tayloristischen, oft von Restriktionen und Repetitivarbeit geprägten Arbeitsbedingungen. Die Zusammenfassung der Beschäftigten in Gruppen macht zwar den Arbeitsplatzwechsel möglich und erhöht den Anteil an unmittelbaren Produktionsaufgaben. Eine qualifiziertere Arbeit durch substantiell erweiterte Aufgabenzuschnitte entsteht auf diesem Weg freilich nicht, da weder indirekte Funktionen noch Aufgaben integriert werden. Arbeitsteilung und Spezialisierung bleiben die prägenden Merkmale der Arbeitsorganisation. Die Selbstorganisation der Teams ist eher gering und beschränkt sich auf gegenseitiges Anlernen, Arbeitseinteilung und in manchen Fällen auch arbeitsvorbereitende Tätigkeiten. Dispositive und planerische sowie Optimierungsaufgaben werden, wenn sie überhaupt in die Gruppen gegeben werden, ausschließlich vom Gruppensprecher übernommen. Dieser ist in der Regel vom Management eingesetzt, trägt die Linienver-

\footnotetext{
${ }^{1}$ Wir werden im folgenden die Begriffe Gruppenarbeit und Teamarbeit synonym verwenden.

${ }^{2}$ Da kaum einschlägige Studien für die Prozessindustrien vorliegen, fassen wir zunächst einige wesentliche Ergebnisse aus den Fertigungsindustrien, insbesondere der Studien aus dem SOFI zusammen, vgl. Gerst et al. 1995; Kuhlmann 1996; Schumann, Gerst 1997; Gerst 1998; Gerst 1999; Schumann, Kuhlmann 2000.

${ }^{3}$ Diese Einordnung stellt nur eine neben vielen anderen dar. Die wissenschaftliche Erforschung der arbeitsorganisatorischen Konzepte führt meist zu dichotomen Darstellungen, Unterscheidungsmerkmale bietet der nationale Vergleich (Japan versus Schweden bzw. Deutschland), das Organisationskonzept (Toyotismus versus Humanisierung; Lean production versus soziotechnischer Ansatz; strukturkonservativ versus strukturinnovativ; modifizierter Taylorismus versus selbstorganisierte Gruppenarbeit), die Orientierung (Produktivität vs. Beteiligung), dazwischen finden sich Kombinationen aus den Merkmalen (Benders 1996). Zur Debatte um den Gestaltungsansatz der standardisierten Gruppenarbeit vgl. Adler, Cole 1993; Adler 1995; Springer 1999, zur Kritik Gerst 2000.
} 
antwortung und ist damit in einer Vorarbeiter-Rolle. Die strikte Hierarchie in der Gruppe schränkt die Spielräume zur Selbstorganisation erheblich ein. Diese Fälle bleiben von daher gekennzeichnet durch eine ausgeprägte betriebliche Statusdifferenzierung sowie durch hierarchische Koordination und Kontrolle.

Das Gestaltungskonzept der selbstorganisierten Gruppenarbeit hingegen sieht einen Bruch mit tayloristischen Mustern der Arbeitsorganisation auf vier Ebenen vor. Erstens ist eine breite Aufgaben- und Funktionsintegration vorgesehen, die darauf zielt, für den einzelnen Arbeitenden einen anspruchsvolleren und interessanteren Arbeitsplatz auf der Basis höherer Qualifikation zu schaffen. Darüber hinaus wird das Spektrum der gemeinsamen Kernaufgaben erweitert und eine Grundlage für kooperatives Arbeiten geschaffen. Im Ergebnis entstehen so Spielräume für organisatorische Alternativen, für gemeinsame Arbeitsausführung und für gegenseitige Unterstützung. Zweitens beruht das Konzept auf gruppengetragener Selbstorganisation, d.h. Entscheidungs- und Gestaltungsbefugnisse werden den Arbeitsgruppen übertragen. Planung und Kontrolle von (Teil) Aufgaben gehen damit in die Regie der Gruppe über, so etwa die Besetzung der Arbeitsplätze oder auch die Programmplanung. An die Stelle einer detaillierten und von den Vorgesetzten vorgegebenen Planung tritt eine auf betriebliche Ziele ausgerichtete Rahmenplanung, in deren Grenzen sich die Freiräume der Gruppenmitglieder bewegen. Unterstützt wird die Selbstorganisation durch eigenverantwortlich gestaltete Gruppengespräche und frei gewählte Gruppensprecher, die als primus inter pares koordinierende und moderierende Funktionen übernehmen. Drittes zentrales Gestaltungsmerkmal ist der Rollenwechsel der Vorgesetzten zu Unterstützern oder „Coaches“ der Arbeitsgruppen und die Etablierung eines partizipativen Führungsstils. Besonders gefordert sind die Meister als unmittelbare Vorgesetzte der Arbeitsgruppen. Sie müssen zwischen betrieblichen Zielen und Vorstellungen der Gruppenmitglieder vermitteln und die Gruppen insbesondere bei Optimierungsaufgaben unterstützen. Dazu gehört etwa die schicht- und bereichsübergreifende Koordination einzelner Projekte, Informationsübermittlung zwischen den beteiligten Akteuren, sowie die Unterstützung der gruppeninternen Qualifizierung und die Vertretung der Interessen der Gruppe nach außen. Schließlich gilt als viertes Element die Veränderung etablierter Kommunikations- und Kooperationsstrukturen, die zunehmend hierarchie- wie fachübergreifend erfolgt, um eine engere Kooperation zwischen den Akteuren in der direkten Produktion und dem betrieblichen Umfeld $^{4}$ zu erreichen. Ziel dieses Gestaltungsansatzes ist es, umfassend die Fähigkeiten und Wissensbestände der Beschäftigten in den Innovationsprozess einzubinden und zu fördern. Eine wesentliche Voraussetzung dafür ist die Nutzung der kreativen Aspekte menschlichen Arbeitshandelns im Sinn von Innovationsarbeit, die in diesem arbeitsgestalterischen Ansatz zum veränderten Anforderungsprofil der Beschäftigten in der Produktion wird. Die Verknüpfung der vier Gestaltungsziele stärkt die Möglichkeiten zum Innovationshandeln der Beschäftigten in besonderer Weise. Aufgabenintegration heißt dann, dass nicht nur die Prozessverantwortung, der Beschäftigten steigt. Zugleich erhöht sich auch das Problemlösungspotential der Gruppen durch die gruppengetragene Selbstorganisation: Sie bildet die Basis für Kommunikation und Kooperation sowohl innerhalb der Gruppe wie auch mit Externen und ermöglicht die Zusammenführung unterschiedlicher Wissensbestände, die für ein effizientes Optimierungshandeln unabdingbar sind. Die veränderte Vorgesetztenfunktion sichert

\footnotetext{
${ }^{4}$ Gemeint ist damit sowohl die Zusammenarbeit auf horizontaler Ebene mit Fachabteilungen wie Instandhaltung, Qualitätssicherung, Logistik, Labor etc. als auch die auf vertikaler Ebene mit betrieblichen Vorgesetzten.
} 
und unterstreicht den neuen Status, der den Beschäftigten zugestanden wird (bzw. der von ihnen eingefordert wird).

\begin{tabular}{|c|c|c|}
\hline & modifizierter Taylorismus & selbstorganisierte Gruppenarbeit \\
\hline $\begin{array}{l}\text { unmittelbare } \\
\text { Produktionsaufgaben }\end{array}$ & $\begin{array}{l}\text { Rotation, hohe Einsatzflexibilität; } \\
\text { Ziel: begrenzter Gesamtumfang }\end{array}$ & $\begin{array}{l}\text { Rotation, hohe Einsatzflexibilität; } \\
\text { Ziel: erweiterter Gesamtumfang }\end{array}$ \\
\hline indirekte Aufgaben & $\begin{array}{l}\text { geringer Umfang, stattdessen Einsatz von } \\
\text { Spezialisten }\end{array}$ & $\begin{array}{l}\text { erweiterter Umfang, weitgehende } \\
\text { Rücknahme von Spezialisierung }\end{array}$ \\
\hline Selbstorganisation & $\begin{array}{l}\text { eingeschränkt: Entscheidungen treffen } \\
\text { Meister und Gruppensprecher }\end{array}$ & $\begin{array}{l}\text { erweitert: Planung und Kontrolle als } \\
\text { Aufgabe der Gruppe }\end{array}$ \\
\hline Gruppensprecher & $\begin{array}{l}\text { Sonderstatus durch Umfeldaufgaben } \\
\text { und Freistellung, oft eingesetzt und } \\
\text { weisungsbefugt }\end{array}$ & $\begin{array}{l}\text { gleichberechtigtes Gruppenmitglied, } \\
\text { gewählter Koordinator und Ansprechpartner }\end{array}$ \\
\hline Meister & $\begin{array}{l}\text { weiterhin starke hierarchische Position, } \\
\text { Führung durch Anweisung und Kontrolle }\end{array}$ & $\begin{array}{l}\text { Unterstützer der Gruppe, vermittelt } \\
\text { zwischen betrieblichen Zielen und } \\
\text { Gruppeninteressen }\end{array}$ \\
\hline Gruppengespräche & $\begin{array}{l}\text { thematische Engführung auf betriebliche } \\
\text { Information und Produktivität }\end{array}$ & freie Themenwahl, eigene Gestaltung \\
\hline Leistungspolitik & $\begin{array}{l}\text { Leistung durch Standardisierung, } \\
\text { Anweisung und Kontrolle }\end{array}$ & Leistung durch Eigeninitiative und Kreativität \\
\hline $\begin{array}{l}\text { Innovations- und } \\
\text { Optimierungsaufgabe }\end{array}$ & $\begin{array}{l}\text { Geringer Stellenwert; KVP getragen } \\
\text { von Spezialisten; Ziel: Optimierung und } \\
\text { Standardisierung der Arbeitsausführung }\end{array}$ & $\begin{array}{l}\text { Hoher Stellenwert; getragen von der } \\
\text { gesamten Gruppe; Ziel: Optimierung des } \\
\text { gesamten sozio-technischen Systems }\end{array}$ \\
\hline
\end{tabular}

Quelle: Gerst 1998; eigene Ergänzungen;

Abbildung 1: Konzepte von Gruppenarbeit

In der Konzeptionskonkurrenz der 1990er Jahre setzt sich zunächst die selbstorganisierte Gruppenarbeit als neuer Leittypus durch, und Gewerkschaften wie Beschäftigte goutieren diesen neuartigen Ansatz, sichert er doch sowohl den Unternehmen Effizienz als auch den Beschäftigten qualifiziertere und interessantere Arbeit. Neue Elemente wie dispositive, soziale und kommunikative Kompetenzen forcieren die Metamorphose der Beschäftigten vom Objekt zum Mitgestalter vor Ort (Schumann, Gerst 1997) und damit zum „manifesten Leistungsträger“ (Voß, Pongratz 1998:141). Bezogen auf die Leistungssituation heißt dies aber auch: Erweiterte Spielräume zur Leistungsregulation, interessantere Arbeitsinhalte und die neuen sozialen, kommunikativen und selbstorganisatorischen Herausforderungen, die der Betrieb im Rahmen der prozessorientierten Organisation an die Beschäftigten stellt, sind in diesen Projekten nicht ohne höhere Arbeitsbelastungen und Anforderungen zu haben. In ihren Wirkungen bleibt die gruppengetragene Selbstorganisation von daher ambivalent. ${ }^{5}$ Denn keineswegs geklärt ist die Frage, ob eine Verlagerung von planenden, steuernden und regulierenden Funktionen auf die Ebene des Shop floor, also in die Gruppen hinein, gleichzusetzen ist mit einem Mehr an Autonomie für die Beschäftigten bzw. wie und ob es den Beschäftigten gelingen kann, ihre formalen Autonomiegewinne auch zur Durchsetzung eigener Interessen zu nutzen. Konkret geht es in der Debatte um die Frage, inwiefern sich die Beschäftigten dagegen wehren können, dass ihr Wissen

\footnotetext{
${ }^{5}$ Vgl. Döhl et al. 2000, Gerst 1999; 2002; Kuhlmann, Schumann 2001; Moldaschl 1994; 1996; 1999; Wolf 1999.
} 
und Können, ihre Kreativität, Bildung, Intelligenz und Erfahrung, ihr persönliches Engagement bis hin zu ihrer Zeiteinteilung im Rahmen innovativer Arbeitspolitik für die betrieblichen Zwecke vereinnahmt wird. Handelt es sich bei den neuen Arbeitsformen nicht um eine historisch gesehen beinah als kontinuierlich zu bezeichnende Ausweitung der Vernutzung von Arbeitskraft?

Möglichkeiten zur direkten Partizipation können sehr wohl den Weg zu einer quasitotalitären betrieblichen Organisation ebnen. ${ }^{6}$ Flankiert von einer dezentralisierten und flexibilisierten Produktion forcieren die Unternehmen einen tiefgreifenderen Eingriff in die Subjektivität der Beschäftigten, indem sie neue Spielräume für autonomeres Arbeitshandeln gewährleisten. Autonomie wird dann zu einem neuartigen Kontrollinstrument, das auf der Beherrschung der Gesamtheit der Orientierungen und Bedürfnisse der Beschäftigten im Sinn betrieblicher Ziele basiert. Statt direkter, technisch vermittelter und bürokratischer Kontrollformen, die von außen auf den einzelnen Beschäftigten wirken, wird nun auf Basis betrieblicher Zielvorgaben eine selbstorganisierte Selbstkontrolle der Beschäftigten eingeführt. Die Kontrolle des Managements vermittelt sich dann einerseits durch die von den Arbeitenden übernommenen Normen und Wertvorstellungen, andererseits erleichtern (informations-) technologische Möglichkeiten die indirekte Kontrolle der Arbeitsleistung. Gerst (2000) hat für Vertreter dieser theoretischen Perspektive eine „Repressionsthese“ formuliert, die ihre Ursprünge bereits 1984 bei Türk findet. In Anlehnung an die Marx'schen Begriffe der formellen und reellen Subsumtion ${ }^{7}$ werden die durch die neuen Konzepte implizierten Veränderungen des konkreten Arbeitsprozesses als ideelle Subsumtion interpretiert, in deren Verlauf sich die Subjekte fremde Interessen zu eigen machen. Ähnlich argumentiert Deutschmann (1989), der auf die kulturimperialistische Vorgehensweise des Managements gegenüber den Beschäftigten verweist. Dass die Beschäftigten nun bereit sind, die vom Betrieb positiv konnotierten erweiterten Gestaltungsund Handlungsspielräume auszuschöpfen und dabei Widerstandspotentiale gegen überzogene Leistungsansprüche allzu schnell aufgegeben würden, betonen vor allem gewerkschaftliche Beiträge (Peters 1997; Glissmann 1999). Und schließlich wird gruppengetragene Selbstorganisation auch systemimmanent bzw. bezogen auf die strukturellen Aspekte von Industriearbeit kritisiert. In dem Maß, in dem Beschäftigte ihre Arbeit in einer fremdbestimmten Arbeitsumgebung verrichteten, diene jedes Mehr an Autonomie auf dem Shop floor letztlich einer Ausweitung von betrieblicher Herrschaft. Denn jedes Handeln sei in diesem Rahmen an den betrieblichen Interessen und Leistungszielen orientiert, Autonomie und Selbstorganisation blieben ideologisch und euphemistisch eingesetzte Begriffe ohne reale Entsprechung. ${ }^{8}$

\footnotetext{
${ }^{6}$ Die folgende dichotome Darstellung geht zurück auf Gerst (2000, 10ff).

${ }^{7}$ Formelle Subsumtion bedeutet, dass zunächst nicht unter dem Kapitalverhältnis stattfindende Arbeitstätigkeiten diesem untergeordnet werden, ohne aber die Tätigkeit selbst substantiell zu ändern. Aufbauend auf der formellen setzt die reelle Subsumtion ein und verändert die Arbeitstätigkeit und die Produktionsmittel so, dass sie den Verwertungsprinzipien des Kapitals entsprechend am effizientesten genutzt werden. Die reelle Subsumtion hat einen Disziplinierungsprozess der Arbeit zur Folge, mit dem Effekt, dass „die Regelmäßigkeit, Gleichförmigkeit, Ordnung, Kontinuität und Energie der Arbeit wundervoll erhöht“ werde (Marx, Das Kapital I, in: MEW Bd. 23, 1986, S. 433).

${ }^{8}$ In diese Richtung argumentieren etwa Moldaschl (1994; 1996), Wolf (1999), Gorz (1989; 2000), Pongratz, Voß (1997).
} 
Mit der sogenannte Emanzipationsthese (Gerst 2000) wird hingegen betont, dass die Reorganisation der Arbeit im Sinn innovativer Arbeitspolitik eine Entfremdung in der Arbeit zwar nicht aufheben, ihre negativen Folgewirkungen aber abschwächen könnte. Die Formalisierung der informellen Aushandlungsprozesse durch gruppengetragene Selbstorganisation trage zwar zu einer Verschärfung der Belastungen bei, die sich nicht allein aus den allgemein mit Gruppenarbeit assoziierten erhöhten Leistungsanforderungen durch eine auch objektiv vorhandene Arbeitsverdichtung erklärt. Die Leistung des Einzelnen würden jetzt verstärkt von der Gruppe kontrolliert und konnten Fehler bzw. auch der Umgang mit ihnen früher auf „die da oben“ geschoben werden, müsse nun in vielen Fällen das Team dafür gerade stehen und sich Lösungsmöglichkeiten überlegen. So sei es wenig erstaunlich, dass sich etwa in empirischen Studien die Befragten stark belastet fühlen durch die Gefahr, Fehler zu machen und den Zwang zur Konzentration - und dieser Anstieg auf die ansonsten durchaus positiv bewertete Teamarbeit zurückgeführt wird. Die Ergebnisse entsprechen den Überlegungen von Gerst (2002), der diesen Prozess als eine „Instrumentalisierung der Solidargemeinschaft“ bezeichnet: Der Betrieb verlässt sich auf die in den Schichtmannschaften bereits angelegten Formen des solidarischen Handelns, die den Einzelnen nach außen schützen aber intern bestimmte Anforderungen im Hinblick auf die Erfüllung betrieblicher Leistungsnormen beinhaltet. Doch müssen diese gruppeninternen Kontrollmechanismen nicht zwingend negativ bewertet werden. Im Gegenteil: Erhöhte Leistungsanforderungen werden von den Beschäftigten dann akzeptiert, wenn Teamarbeit insgesamt zu einer Verbesserung ihrer Arbeitssituation beiträgt und die Leistungsverdichtung zu einer zu vernachlässigenden Nebenfolge wird (Gerst et al. 1994; Schumann, Gerst 1997; Kuhlmann, Schumann 2000; Gerst 2002). Hervorzuheben sei daher der emanzipatorische und demokratische Aspekt der neuen Arbeitsformen und die damit verbundenen Chancen zu einer Reprofessionalisierung sowie zu solidarischem Handeln (Kuhlmann, Schumann 2000). Das Umstellen von hierarchischer Steuerung auf Steuerung mittels Zielvorgaben ${ }^{9}$ biete die Möglichkeiten zu einer auf Kommunikation basierenden diskursiven Koordinierung (Braczyk, Schienstock 1996; Braczyk 1997). Neu ist nicht die Tatsache, dass kommuniziert wird, sondern die sowohl qualitativ als auch quantitativ bestimmbare Veränderung der Relevanz von Kommunikation. Diskursivität deutet einmal an, dass ein Austausch von Argumenten, also eine Form von Verhandeln stattfinden kann und an die Stelle einer top down verlaufenden „Ein-WegKommunikation“ (Minssen 2001) tritt. Möglich sind nun alle Formen von Kommunikation und zwar unabhängig von der hierarchischen Position des Sprechenden. Neben die top down Variante wird damit auch die bottom up-Kommunikation möglich, die vertikale um die horizontale Ebene ergänzt. Auf dem Shop floor findet damit eine einschneidende Veränderung statt. Galt Kommunikation dort bislang als kontraproduktiv und wurde offiziell als notwendiges Übel geduldet, werden die kommunikativen Kompetenzen der Beschäftigten nun zum Teil des betrieblichen Anforderungsprofils. Gruppensprecher und Gruppengespräche verweisen auf diesen veränderten Stellenwert und unterstreichen den Aspekt der Selbstorganisation im Sinn von Abstimmung und Diskussion auf Gruppenebene.

\footnotetext{
${ }^{9}$ Ganz bewusst wird an dieser Stelle begrifflich auf Zielvorgaben und nicht Zielvereinbarungen rekurriert, da sich in der betrieblichen Praxis noch jede Vereinbarung unter der Hand oder auch sehr offen seitens des Managements in eine Zielvorgabe umwandeln lässt.
} 
Die Emanzipationsthese fasst Kommunikation im Sinn der „Theorie des kommunikativen Handelns“ (Habermas) ${ }^{10}$ auf. Diese bilde die Grundlage für einen betrieblichen Demokratisierungsprozess, der in der optimistischen Auslegung von Dubiel (1997) seitens des Managements nicht einfach zurückzunehmen sei. Zwar gilt, dass sich die Leistungsanforderungen verschärfen und Interessenkonflikte weiterhin bestehen. Die neuen kommunikativen Regeln und die Ausweitung der Ressourcen zur Selbstorganisation stärken aber die innerbetriebliche Position der Beschäftigten, so dass sie aktiv Bewältigungsstrategien für die Belastungssituation entwickeln können, und auch alternative Vorschläge (etwa in Fragen der Ergonomie) offensiv gegenüber dem Management vertreten - und durchsetzen, dies belegten auch Befragungsergebnisse (Schumann 2002). ${ }^{11}$

Auf Basis der vorliegenden Befunde ist empirisch evident, dass die Arbeitssituation in selbstorganisierter Gruppenarbeit von den Beschäftigten als deutlich verbessert gegenüber der traditionellen Arbeitsgestaltung beurteilt wird. Insgesamt ist in den untersuchten Fällen ein höheres Maß an Einverständnis mit den betrieblichen Leistungszielen auszumachen (Gerst 1999; Schumann, Kuhlmann 2000). Hinreichend geklärt sind darüber hinaus die arbeitsorganisatorischen Bedingungen, unter denen die vorhandene Produktivitätsund Innovationspotentiale der Beschäftigten sich entfalten können. Fraglich bleibt aber, wieso sich das arbeitsgestalterische Konzept der selbstorganisierten Gruppenarbeit nicht durchsetzen konnte, denn weder kann aus Unternehmenssicht eine ,negative Leistungsbilanz“ bezogen auf diese Arbeitsform gezogen werden, noch ist ein Widerstand der Beschäftigten empirisch nachzuweisen.

Ein möglicher Erklärungsansatz ist, dass die seit Mitte der 1990er Jahre zunehmende Orientierung der Unternehmen am Shareholder Value die auf arbeitsorganisatorische Innovationen setzende Variante verdrängt ${ }^{12}$. Als neues Leitbild effektiver Produktion werden vor allem konservative arbeitsorganisatorische Lösungen propagiert, da Produktivitätssteigerungen, Optimierungen und Kostensenkung durch diese arbeitsgestalterischen Ansätze in kürzeren Fristen zu realisieren sind (Jürgens 1997; Schumann 1998; Gerst 1999; Springer 1999). Selbstorganisation, Funktionsintegration und Qualifizierung behindern in dieser Perspektive diese Zielsetzung, da sie zunächst hohe Kosten verursachen und die Leistungs- und Effizienzvorteile im Rahmen vorhandener betrieblicher Controlling- und Messverfahren nicht abbildbar sind. Das Konzept selbstorganisierter Gruppenarbeit kann

\footnotetext{
${ }^{10} \mathrm{Zu}$ der Frage, inwieweit die Gruppenprozesse mit der Habermasschen Theorie des kommunikativen Handelns vereinbar sind vgl. Parge 2003.

${ }^{11}$ Unter den Prämissen der Repressionsthese bleibt Kommunikation nach wie vor ein Mittel zur Koordination und damit orientiert an zweckrationalem und normorientierten Handeln - also dem betrieblichen Interesse unterworfen. Der Betrieb gilt weiterhin als Sphäre der Heteronomie, und Hinweise auf ein von den Beschäftigten positiv bewertetes Mehr an selbstorganisiertem Handeln auf dem Shop floor wird als „Verblendung“ oder „falsches Bewusstsein“ interpretiert.

12 Die Bezeichnung „innovativ“ ausschließlich für die zweite Variante ist insofern irreführend, als der Begriff immer die Einführung von etwas Neuem beinhaltet. Auch eine Modernisierung des Taylorismus ist insofern als Neuerung zu verstehen. In der Innovationsforschung würde man wohl für den ersten Fall die „inkrementelle“ Innovation gebrauchen, während die zweite Variante aufgrund ihres umfassenderen Charakters eher als „,radikale“ Innovation zu fassen wäre. In der industrie- und arbeitssoziologischen Literatur hat sich die Verwendung des Worts „,innovativ“ im Bezug auf die Arbeitsorganisation für die Fälle durchsetzen können, in denen Unternehmen und Beschäftigte gleichermaßen von den Reorganisationsmaßnahmen profitieren. Wir werden uns im folgenden an diese allgemein übliche Sprachregelung halten.
} 
genau mit diesen schnellen Einspareffekten nicht aufwarten, im Gegenteil, es bindet gerade in der Einführungsphase zusätzliche Ressourcen.

Insbesondere in den Handarbeitsbereichen der Montagen bedeutet Gruppenarbeit für die Beschäftigten dann eine weitere Verschärfung der ohnehin von Restriktionen geprägten Arbeitssituation. Montagezyklen werden bis an die Grenze der Belastbarkeit verkürzt, Standardisierung und Hierarchie gewinnen an Bedeutung. Dass diese psychischen wie physischen Mehrbelastungen die Arbeitskräfte auf Dauer schnell und nachhaltiger vernutzen, scheint Unternehmen in Zeiten hoher Arbeitslosenquoten nicht weiter zu interessieren. Allerdings stellt eine Aufwertung von Produktionsarbeit in diesen Bereichen nicht nur aus den genannten ökonomischen Gründen keine wirkliche Alternative dar. Für die Montage standardisierter Massenprodukte ist innovatorisches Handeln der Beschäftigten in dem Maß, wie es Konzepte selbstorganisierter Gruppenarbeit vorsehen, kein zentraler Stellhebel im Rationalisierungsprozess. Vielmehr gilt hier die Maxime, die geforderten Stückzahlen möglichst schnell bei hoher Qualität zu erzielen. ${ }^{13}$ Die Produktionsintelligenz und das Erfahrungswissen der Beschäftigten werden dabei nur im minimalen Ausschnitt des einzelnen, qualifikatorisch wenig anspruchvollen Arbeitsplatzes benötigt. Optimierungshandeln bezieht sich in diesem Konzept nicht auf größere Projekte zur Produktoder Prozessinnovation, sondern ist fokussiert auf die Details des einzelnen Arbeitssystems. In komplexe Rückkopplungsprozesse und querfunktionale Verknüpfungen, die über die arbeitsorganisatorischen Anforderungen der direkten Produktion hinaus weisen, sind die Beschäftigten in diesem unternehmerischen Ansatz nicht mit einbezogen.

Dass dieses keineswegs die Rückkehr zum Taylorismus (Springer 1999) bedeutet und sich Arbeitspolitik nicht nur im Rückwärtsgang bewegt (Gerst 1999), belegt ein Blick auf Konstellationen in den automatisierten, hochtechnisierten Bereichen der Produktion. Hier profitieren die Beschäftigten häufiger von einer auch zu ihren Gunsten veränderten Arbeitsorganisation, deren Basis selbstorganisierte Gruppenarbeit bildet. Im Rahmen der Einführung von selbstorganisierter Gruppenarbeit wird in diesen Bereichen eine systematische, organisatorische Verzahnung der Steuerungs-, Herstellungs-, Überwachungs- oder Regulierungsaufgaben mit einer Innovations- und Optimierungsfunktion, d.h. erweiterten Anforderungen beim Aufdecken, Aufzeigen und dauerhaften Beseitigen von Störungsquellen und Problemen in technischen wie organisatorischen Abläufen, vorgenommen. Das Besondere ist, dass die genaue Definition des Problems selbst ein wesentlicher Bestandteil der Arbeitsaufgabe wird, mit erheblichen und den Entwicklungen im Angestelltenbereich durchaus vergleichbaren Folgen für das Anforderungsprofil. Denn das routinierte und berufsfachlich geprägte Handlungsrepertoire ist für die Bewältigung dieser Aufgaben nicht mehr ausreichend, es müssen zusätzlich fachübergreifende Kenntnisse und Expertisen einbezogen werden. Die höhere Flexibilität in der Produktion konfrontiert die Beschäftigten mit mehr Produkten, Prozessen, Verfahren, so dass auch die Optimierungsprozesse selbst systemisches Denken erfordern. Eigene Wissensbestände werden dann permanent mit denen anderer Kollegen aber auch anderer Fachbereiche wie Hierarchieebenen abgeglichen, verknüpft und ergänzt. Die kommunikativ-kooperativen Fähigkeiten der Beschäftigten spielen hier eine besondere Rolle, da sie nun innerhalb des Arbeitssystem wie auch hierarchieübergreifend als Grundlage für Lernprozesse gelten. Alles

\footnotetext{
${ }^{13}$ Die Studie von Kurz (1999) widmet sich dieser Verknüpfung tradierter und neuer Bezugspunkte betrieblicher Rationalisierung und deren Wirkeffekten für Repetitivarbeit und den modernen tayloristischen Massenarbeiter.
} 
in allem ist ein erweitertes Verständnis für technische, organisatorische und ökonomische Belange gefordert, auch wenn die eigenen Kooperationsbeiträge weitgehend auf die ausbildungsbegründete Spezialistenrolle begrenzt bleibt (Schumann 2002).

Die diametral entgegengesetzten Entwicklungen in den manuellen und technisierten Bereichen werden vielfach damit erklärt, dass für avancierte technisierte Produktionssysteme eine Desynchronisation von maschineller und menschlicher Arbeitsleistung charakteristisch ist. Sie lässt mehr Spielräume bei der Arbeitsgestaltung, als es etwa der zeitlich unflexiblere Rhythmus des Fliessbands erlaubt. Gegen eine solche rein technikbasierte Interpretation sprechen hingegen Befunde zur durchaus problematischen, aber letztlich sowohl unter wirtschaftlichen wie sozialen Aspekten positiv bilanzierten Umsetzung selbstorganisierter Gruppenarbeit auch in manuellen Fertigungsbereichen (Gerst 1998). So weist etwa Kurz (1999) in ihrer Studie nach, dass ein innovativer Gestaltungsansatz nicht zu einer Neufassung des repetitiven Arbeitstyps führen kann. Ein substantieller Wandel ist auch mit einer Entdramatisierung der klassischen Gefährdungslagen und dem Mehr an Selbststeuerung nicht zu haben, denn einer konsequent prozessorientierten Nutzung von Wissen und Kenntnissen der Arbeitskräfte stünden die kurzgetakteten, standardisierten Arbeitsabläufe entgegen. Dennoch sei selbstorganisierte Gruppenarbeit ein Stellhebel, der das Arbeitsleid der Beschäftigten deutlich verringere (ebd.). Und dass die in technisierten Bereichen gegebenen Voraussetzungen organisatorisch nicht zwingend mit innovativen Konzepten einher gehen, belegten bereits die Ergebnisse des Trendreport Rationalisierung im Zusammenhang mit den Neuen Produktionskonzepten (Schumann et al. 1994).

Technisierung legt also, so die Befunde, bestimmte Organisationsformen nahe, erzwingt sie aber nicht. Den Betrieben stehen vielmehr mehrere Wege offen, gemeinsamer Nenner der empirisch vorfindbaren Lösungen ist „in jedem Fall die Suche nach einer Lösung, die einen möglichst störungsfreien und optimierten Prozessablauf garantiert“ (Schumann et al. 1994:646). Von besonderer Bedeutung für die Ausrichtung arbeitsgestalterischer Maßnahmen auf dem Shop floor ist demnach, ob das Unternehmen von einem stofflichtechnisch stabilen lokalen Produktions- und Arbeitsprozess ausgeht, oder ob dieser als instabil und durch Unsicherheit in Bezug auf die genannten Anforderungen charakterisiert ist. In systemtheoretischer Hinsicht ${ }^{14}$ bedeutet dies, dass die Reflexion des Systems „Unternehmen“ über die Bestimmung der Systemidentität (= Innovations- und Produktionsmodell) mit darüber entscheidet, welches die umweltrelevanten Aspekte (= Reagibilitäts, Flexibilitäts- und Innovationsanforderungen) sind. Diese unternehmensinterne Definition der Umweltbedingungen wiederum beeinflusst maßgeblich die Regeln (verstanden als arbeitsgestalterische Ansätze), die sich das System „Unternehmen“ bei der Bearbeitung des durch die Umwelt entstehenden Problems der Komplexitätsreduktion auferlegt. ${ }^{15}$

\footnotetext{
${ }^{14}$ Ein Verweis liegt insofern nahe, als die organisierte und strukturelle Komplexität (sozialer) Systeme die zentrale Problemstellung der Systemtheorie ist und moderne Gesellschaften vor dem Problem stehen, diese durch Prozesse von Selbstorganisation, Kontrolle und Steuerung zu bearbeiten (Luhmann 1998; Willke 1998).

${ }^{15}$ Damit dürfte deutlich werden, dass nicht die Umwelt komplexer geworden ist, sondern lediglich die Definition der Komplexität aus Sicht der Unternehmen sich verändert hat. Durch die paradoxe Situation, dass mit dem Begriff Komplexität die Einheit einer Vielheit ausgedrückt werden soll, ist es nicht möglich, Komplexität in quantitativen Relationen gegeneinander zu wenden. Luhmann selbst stellt als Konkretisierung dieses Paradoxons die Frage: „Ist ein Gehirn komplexer als eine Gesellschaft, weil es in einem Gehirn mehr Nervenzellen als Menschen in einer Gesellschaft gibt?“ (Luhmann 1998:137)
} 
Festzuhalten ist: In diesem paradigmatischen Verständnis der Unternehmensorganisation wird das spezifische menschliche Arbeitsvermögen, das in der Auseinandersetzung mit einem stofflich-technisch stabilen und „lokalen“ Produktions- und Arbeitsprozess, d.h. der unmittelbaren Arbeitsumgebung entsteht (Produktionsintelligenz) und dessen Produktivkraft sich im Rahmen erweiterter Handlungsspielräume der Arbeitskräfte entfaltet, zum zentralen Betzugspunkt. Allerdings ist die Umsetzung arbeitsgestalterischer Ansätze, die auf selbstorganisierter Gruppenarbeit basieren, in Unternehmen insbesondere dann zu erwarten, wenn der Einsatz der Produktivkraft menschlichen Arbeitsvermögens notwendig erscheint, um die Reagibilitäts, Flexibilitäts- und Innovationsanforderungen des dynamischen Markts (Baethge, BaethgeKinsky 1998:42) wie auch der technischwissenschaftlichen Entwicklungen zu bewältigen. 


\section{Innovations- und Produktionsmodell der Chemischen Industrie}

Den Hauptgrund für die „Sperrigkeit“ der Chemischen Industrie gegenüber einem tayloristischen Rationalisierungszugriff und - damit verbunden - gängigen Erklärungs- und Interpretationsfolien der Rationalisierungsanalyse sehen Studien, ${ }^{16}$ die sich mit der Entwicklung der Chemischen Industrie bislang auseinandergesetzt haben, in erster Linie in der stofflich definierten Prozesshaftigkeit der Produktion, die eine Zergliederung der Arbeitsfunktionen nach den Grundsätzen der wissenschaftlichen Betriebsführung nicht oder allenfalls partiell zugelassen hat. Wesentliches Bestimmungsmerkmal für die Spezifität der Chemischen Industrie ist die der Branche zugrunde liegende problematische Definition ihres Produkts, denn die Chemische Industrie deckt ein weites und überaus heterogenes Produktspektrum ab. Basieren Verkaufsprodukte der Chemie noch auf einer durchaus überschaubaren Anzahl von Vorstufen - Erdöl, Erdgas, Kohle und Erze -,werden diese schon zu etwa 300 chemischen Grund- und Zwischenprodukten ${ }^{17}$ veredelt, die wiederum Grundlage für bis zu 30.000 für den Markt produzierte Endprodukte sind. „Das“ Endprodukt, wie es sich etwa in der Automobilindustrie bei aller Diversifizierung noch unzweifelhaft erkennen lässt, existiert in der Chemischen Industrie nur in einer sehr abstrakten Form. Für die Marktstrukturen bedeutet dies, dass die Zergliederung in eine Vielzahl von Produktsparten und Einzelerzeugnisse die Entstehung von Einzelmärkten forciert, die untereinander nur geringen oder keinen Bezug zueinander haben. Zwar ist beispielsweise auch der Automarkt differenzierbar in Nutzfahrzeuge, Kleinwagen, Mittelklasse und Luxuslimousinen, doch bringt der Automarkt ansatzweise vergleichbare Bedürfnisse auf der Nachfrageseite mit einem überschaubaren Angebot überein. „Der“ Markt für Chemieprodukte ist hingegen so heterogen, dass eine Betrachtung des Ganzen immer in der Gefahr einer (unzulässigen) Stilisierung steht. ${ }^{18}$ Und auch für die einzelnen Produktionsprozesse ist eine einheitliche Aussage kaum zu treffen, da die Möglichkeiten zur inner- wie überbetrieblichen Verknüpfung von Produktlinien unterschiedliche kontinuierliche und diskontinuierliche Verfahren der chemischen Stoffumwandlung wie auch der physikalischmechanischen Stoffverformung erfordern. Monoproduktanlagen, in denen Stoffe in einem kontinuierlichen Herstellungsprozess produziert werden, sind ebenso verbreitet wie Mehrzweckbetriebe, in denen die einzelnen Verfahrensschritte nacheinander, d.h. diskontinuierlich laufen. Im Gegensatz zu Monoanlagen mit einer festen Verknüpfung von Rezeptschritten und Ausrüstungen, werden in einer Mehrzweckproduktion dieselben Ausrüstungen variabel von verschiedenen Rezeptschritten belegt. Die stetig fortschreitende und umfassende Segmentierung der Produktion in Vor-, Zwischen und Endprodukte durch kontinuierliche Produkt- und Verfahrensinnovation prägt dabei das Bild der Chemischen Industrie in besonderem Maß. Die Produktionsstrukturen weisen im Kontrast dazu eine hohe Interdependenz auf, die maßgeblich vom Konzept der Verbundproduktion geprägt ist. Statt einzelner technologischer Kerne der Organisation und daraus folgender vertikaler Verkettung, findet sich in der Chemischen Industrie eine laterale Kombination

\footnotetext{
${ }^{16}$ Blauner 1964; Kern, Schumann 1970; Mickler u.a. 1976; Kern, Schumann 1984; Pries u.a. 1989; Schumann u.a. 1994;

${ }^{17}$ Diese Stoffe werden als „Unsterbliche“ bezeichnet, da sie über Jahrzehnte ihre Bedeutung behalten und sich lediglich sowohl die Verfahren als auch die für die Herstellung notwendigen Rohstoffe verändern.

${ }^{18}$ Ein Problem, dass im Rahmen dieser Arbeit nicht zu lösen ist. Im folgenden werden wir also versuchen, ein Niveau mittlerer Allgemeinheit zu erreichen und die für unsere Fragestellung relevanten Aspekte mit der nötigen Differenziertheit ausleuchten.
} 
verschiedener technologischer Kerne im Rahmen einer Organisation (Tacke 1997). Für die Unternehmensorganisation impliziert dies eine erhebliche Abhängigkeit der einzelnen Produktionsstätten (Betriebe): Losgrößen-, Ablauf-, Termin-, Ressourceneinsatz- und Kapazitätsplanung müssen simultan erfolgen, und die kurzfristige Produktionsmengen und Energiebedarfe ebenso abgestimmt werden wie die langfristige Unternehmensplanung. Eine Linearisierung des Ablaufs ist sowohl in zeitlicher wie auch räumlicher Perspektive vom technologischen Potential her wie aus Gründen der Marktvarianz nicht möglich. Für die einzelbetriebliche Ebene folgen daraus durchaus widersprüchliche Anforderungen. Können Chemiebetriebe einerseits als „Monaden“ bezeichnet werden, da sie über ein hohes Maß an Autonomie im Bezug auf Technik- und Personaleinsatz verfügen, sind sie andererseits immer auch den Zwängen ausgesetzt, die sich aus der Verbundproduktion ergeben. So kann es in ein und demselben Betrieb eines Unternehmens zu einem unterschiedlichen Kosten- und damit Rationalisierungsdruck kommen und zwar in Abhängigkeit von der Marktkonkurrenz und den Möglichkeiten, Verluste durch andere im gleichen Betrieb produzierte Stoffe auszugleichen.

Bei aller Relevanz dieser „stofflich“ argumentierenden Erklärungen ist in diesem Zusammenhang bislang allerdings unterbelichtet geblieben, welche Rolle die Wissensbasierung für die Branchenentwicklung insgesamt gespielt hat und heute noch spielt. Denn die Chemische Industrie als Untersuchungsgegenstand ins Zentrum zu rücken, heißt auch, sie in ihrer Spezifik als science-based industry ${ }^{19}$ zu analysieren. Die Wissenschaftsbasierung prägt in besonderer Weise das Verhältnis zwischen Produktionsmodell, hier verstanden als Produktions- und Arbeitseinsatzkonzepte einerseits und Innovationsmodell, also den Formen der Organisation von Innovationsprozessen andererseits und ist in ihrer technisch-organisatorischen Umsetzung folgenreich für das Kooperations-, Status- und Kompetenzmodell der Branche. ${ }^{20}$ Um Perspektiven von Arbeit in der Chemischen Industrie auszuloten, ist von daher ein historischer Rückgriff notwendig, der die enge Verknüpfung von Wissenschaft und Industrie ${ }^{21}$ (2.3) in Zusammenhang setzt mit spezifischen Rationalisierungsverläufen und dem daraus resultierenden Kompetenzmodell. Es wird zu zeigen sein, dass trotz der chemiespezifischen Verzahnung von Innovations- und Produktionsbereich bis in die Produktion hinein Modi der Übersetzung zwischen den beiden Sphären fehlen und eine iterativ-rekursive Bezugnahme der betrieblichen Akteure im Rahmen von Innovationshandeln ${ }^{22}$ systematisch verhindert wird (2.4). Voranstellen werden wir einige Eckdaten, die das Produktionskonzept der Chemischen Industrie und dessen Wandel aus ökonomischer Perspektive (2.1), sowie die stofflich-technische Entwicklung der Chemieproduktion (2.2) beleuchten.

\footnotetext{
${ }^{19}$ Der Begriff geht zurück auf Noble, der die science-based-industry definiert als "industrial enterprise in which ongoing scientific investigation and the systematic application of scientific knowledge in the process of commodity production have become routine parts of the operation.[...]” (Noble 1977:5).

${ }^{20}$ In Anlehnung an Baethge und BaethgeKinsky (1998) sind die „betrieblich institutionalisierten Regeln und eingeschliffenen Gewohnheiten der Alltagskommunikation“ definiert als das Kooperationsmodell, das ,,abgestufte System von Privilegien und rewards, welche Wertschätzung welche Tätigkeiten und Tätigkeitsgruppen erfahren und welche Macht welche Akteursgruppen im Alltag haben“ als Statusmodell. Wir werden in unserer Analyse diese beiden Aspekte nicht getrennt betrachten, da sich der Akteursstatus in erheblichem Maß auf die Kooperationsformen auswirkt, und diese Wechselbeziehung analytisch für unsere Fragestellung von Relevanz ist.

${ }^{21}$ Für die Chemische Industrie siehe auch Hack, Hack 1985.

${ }^{22}$ Unter Innovationshandeln verstehen wir im folgenden die Ausführung von Planungs-, Steuerungs- und Optimierungsaufgaben.
} 


\subsection{Eckdaten zur ökonomischen Entwicklung}

Der Take-off der Chemischen Industrie in Deutschland ist in der Mitte des 19. Jahrhunderts verorten. Das Gros der deutschen Chemieunternehmen wird zwischen 1859 und 1885 gegründet $^{23}$ und in einer relativ kurzen Zeitspanne von drei Jahrzehnten entwickeln sich die kleinen spezialisierten Betriebe zu großflächigen Produktionsstätten. Die Produktpalette besteht zunächst aus synthetischen Farbstoffen, ${ }^{24}$ die Vor- und Zwischenprodukte bei der Herstellung pharmazeutischer Wirksubstanzen sind. War bis in die 1880er Jahre Großbritannien die stärkste Wirtschaftsmacht in Europa, löst Deutschland durch die „Erfolgsgeschichte“ der Chemie England ab und avanciert bis zum Ersten Weltkrieg zur „Farbenfabrik und Apotheke der Welt““ ${ }^{25}$ (Wetzel 1991:65). ${ }^{26}$

Einen erneuten Expansionsschub erfährt die Industrie durch die Nutzung von Erdöl als neuer Energie- und Rohstoffquelle. Basiert bis zum Jahr 1920 die Weltenergieversorgung auf Kohle, löst bedingt durch die Ausweitung der Erdölproduktion Erdöl sukzessiv die Kohle ab. Zudem kann durch Innovationen im Bereich der organischen Chemie die Produktpalette erheblich erweitert werden. Kunststoffe und synthetische Fasern werden auf der Grundlage von Kohlefolgeprodukten entwickelt. Damit avanciert die Chemische Industrie wirtschaftlich zur Kernindustrie Deutschlands. Schon in den 1870er Jahren arbeiten etwa 50.000 Menschen in der Chemie. Diese Zahl verdoppelt sich innerhalb einer Dekade und erreicht. kurz vor dem Ende des Zweiten Weltkriegs mit beinahe 600.000 Beschäftigten ihren Höhepunkt. ${ }^{27}$

\footnotetext{
${ }^{23}$ So etwa 1859 Boehringer Mannheim; 1863 Bayer, Hoechst und Kalle; 1865 Badische Anilin- und Soda-Fabrik (BASF); 1867 Agfa; 1871 Schering und 1885 Boehringer Ingelheim.

${ }^{24}$ Bereits am Beispiel der synthetischen Farben, also innerhalb einer Produktlinie, lässt sich die exponentiell wachsende Ausdifferenzierung der Produktpalette verdeutlichen. So produzieren die Farbwerke Hoechst im Jahr 1880 (17 Jahre nach Gründung der Fabrik) 1.100 Farbstoffe, sieben Jahre später umfasst die Palette bereits über 10.000 Farben (Christ 1995: 13).

${ }^{25}$ Hier zeichnet sich bereits eine weitere Spezifität der deutschen Chemischen Industrie ab. Während beispielsweise in den USA die Entwicklung der pharmazeutischen Industrie aus den Apotheken und den damit zusammenhängenden Berufen heraus erfolgt, entstehen in Deutschland basierend auf der Färbeindustrie integrierte chemisch-pharmazeutische Unternehmen.

${ }^{26} 1906$ produziert Deutschland mit 4.320 Tonnen beispielsweise vier Fünftel des auf 5.000 Tonnen geschätzten Weltbedarfs an Indigo und exportiert drei Viertel seiner Produktion, auf dem Anilinfarbenmarkt steigt der Anteil Deutschlands am Weltmarkt bis 1913 auf 85 Prozent (Wetzel 1991: 65).

${ }^{27}$ Der Anstieg zwischen 1935 und 1945 ist auf den allgemeinen wirtschaftlichen Aufschwung nach der Weltwirtschaftskrise zurückzuführen. Ab 1936 wirkt sich dann der „Vierjahrplan“ aus, mit dem der „Krieg im Frieden“ vorbereitet wird. Um die Autarkie Deutschlands bei der Herstellung von Treibstoff und Gummi zu gewährleisten, wird die Produktion dieser Stoffe staatlich unterstützt. Und schließlich profitiert die Chemische Industrie im Zweiten Weltkrieg von den nationalsozialistischen „Arbeitsprogrammen“. Die in den Konzentrationslagern internierten Menschen stehen der Großindustrie als kostenlose Arbeitskräfte zur Verfügung. Eine Erhöhung der Beschäftigtenzahl bleibt von daher kostenneutral, ein immenser ökonomischer Vorteil für die deutschen Unternehmen.
} 
Die ökonomische Entwicklung nach dem Zweiten Weltkrieg ${ }^{28}$ ist geprägt durch die Auflösung der wirtschaftlichen und politischen Einheit von den Besatzungsmächten kontrollierte Zonen. Die gesamte Industrie ist von Demontagen und Produktionsbeschränkungen betroffen, darüber hinaus werden Patente und Warenzeichen beschlagnahmt sowie die Verfügungsgewalt über den gesamten ausländischen Patentbesitz von den Alliierten entzogen. Die auch nach 1945 weiter existierenden Strukturen der I.G. Farben stellen dabei für die weitere Entwicklung einen erheblichen Vorteil für die Entwicklung dar, da in diesem Firmenkonglomerat die technologischen wie organisatorischen Grundlagen für die hoch effektive Kuppelproduktion gelegt wurden. ${ }^{29}$ Den immensen Verluste an wissenschaftlichen Vorarbeiten und Know-how verkraftet die deutsche Chemische Industrie von daher schnell und richtet ihre Investitionstätigkeiten vor allem auf eine intensive wissenschaftliche Forschungstätigkeit. Der Anschluss an die scientific community und damit an die internationalen Märkte wird so forciert, und in rascher Folge verbessern und erweitern die Unternehmen ihre Produktionskapazitäten, erfinden und wenden neue Produktionsverfahren an und stellen die Produktion auf kontinuierlich arbeitende Anlagen um. Die Be-

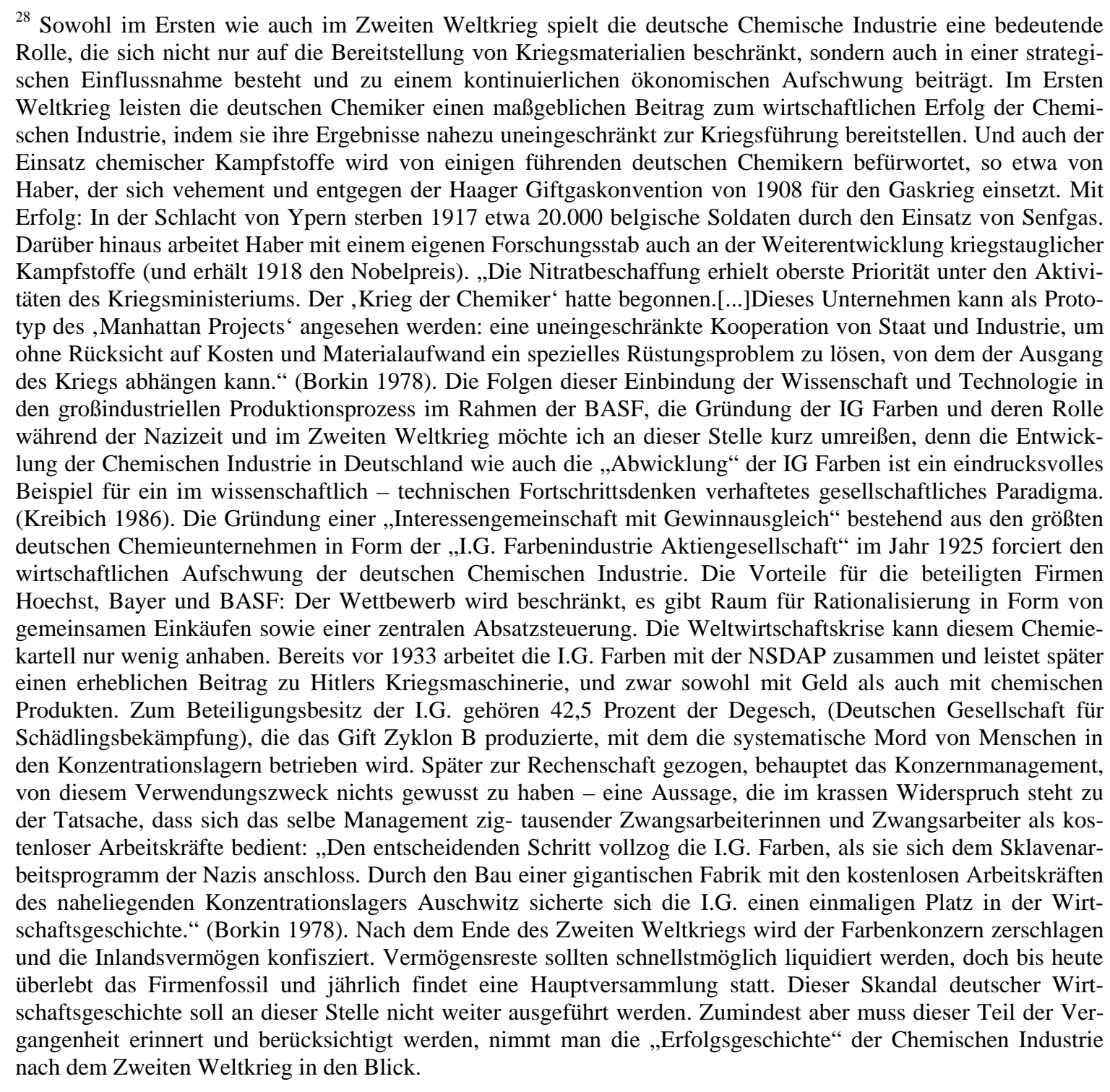

${ }^{29}$ So werden etwa 200.000 Auslandspatente, 24.000 international registrierte Warenzeichen und 200.000 im Ausland eingetragene Schutzmarken entzogen (Schmiede o.J.). 
schäftigtenzahlen verdoppeln sich innerhalb eines Jahrzehnts, der Umsatz verdreifacht sich. ${ }^{30}$

Der Wechsel von der Karbid- zur Petrochemie, den die deutsche Chemische Industrie nach dem Zweiten Weltkrieg vollzieht, forciert den Wachstumsprozess sowohl im Hinblick auf die Produktions- wie die Marktstrukturen. Die großen Chemieagglomerationen (in Leverkusen, Frankfurt a.M. sowie Ludwigshafen) werden über die Einbindung der Raffinerien in die Wertschöpfungskette zu autarken Verbundproduktionsstätten. ${ }^{31}$ Damit sind nicht nur die Bedingungen für einen tiefgreifenden Wandel der Produktpalette der Chemischen Industrie erfüllt, gleichzeitig wandelt sich auch die Stellung der Industrie im Verhältnis zu anderen Branchen. Gilt die Chemie bis in die 1960er Jahre vor allem als Zulieferindustrie, wird sie nun zu einem ihrer wichtigsten Abnehmer.

Einen wissenschaftlichen wie wirtschaftlichen Durchbruch stellt die seit den 1950er Jahren mögliche Substitution natürlicher Rohstoffe wie etwa Holz und Metall durch synthetische Massenware sowohl auf Produkt- wie auf Verfahrensebene dar. Kunststoffe werden neben Chemiefasern, Pflanzen- und Saatenschutzmitteln sowie humanpharmazeutischen Spezialitäten zum wichtigsten Wachstumsträger der deutschen Chemischen Industrie der Nachkriegszeit (Bathelt 1997). ${ }^{32}$ Um die rasch wachsende Nachfrage nach Chemieprodukten zu decken, ${ }^{33}$ setzen die deutschen Unternehmen mit zehn Prozent einen überdurchschnittlich hohen Teil ihres Umsatzes für Investitionen in Anlagen ein, in F\&E fließen ebenso konstant zwischen vier und sechs Prozent des Umsatzes.

Die Expansion der Chemischen Industrie findet parallel zu einem gesamtgesellschaftlichen Wandel statt, der sich in veränderten Gebrauchsgewohnheiten der Abnehmer und Produktionsbedingungen der Anbieter äußert. Bereits nach dem Ersten, vor allem aber seit dem Zweiten Weltkrieg setzt sich in den entwickelten Industriegesellschaften eine Phase kapitalistischer Produktion durch, die ihre Wachstumsdynamik aus der historisch neuen Verbindung von Produktion und Konsum entwickelt (Lutz 1984; Hirsch, Roth 1986; Aglietta 2000). Während zur Zeit der ersten industriellen Revolution vor allem Vorprodukte und Produktionsmittel freigesetzt werden und die gesellschaftliche Reproduktion vorwiegend in der nicht-industriellen Sphäre verbleibt, besetzt das industrielle Kapital mit der Fabrikation von Konsumgütern ein neues Absatzfeld. In diesem als fordistisch bezeichneten Modell findet die Produktion von Gebrauchsgütern im industriellen

\footnotetext{
${ }^{30}$ Im Vergleich zu anderen großen Industriestaaten ist der Produktionszuwachs im Vergleichzeitraum eher gering ausgefallen. Länder wie Großbritannien, Italien, Norwegen und Kanada haben ihren Produktionsausstoß seit 1938 verdreifacht, die USA vervierfacht, während er sich in der BRD „nur“ verdoppelt hat (VCI 1958). Stellt man freilich die Kriegsfolgen in Rechnung, so handelt es sich um einen erstaunlichen, in Anbetracht der Rolle der deutschen Chemischen Industrie im sogenannte Dritten Reich aber erklärbaren Anstieg.

${ }^{31}$ Bereits zwischen 1954 und 1963 steigt der Heizölverbrauch der Chemischen Industrie von knapp 1.7 Mio. Tonnen auf 20.7 Mio. Tonnen. (VCI 1964). Zwischen 1960 und 1970 sinkt der Kohleverbrauch um 50 Prozent, im Vergleichszeitraum erhöht sich der Heizölkonsum um 381 Prozent.

${ }^{32}$ Eine wichtige Rolle in diesem Substitutionsprozess spielt die Preisentwicklung, die in der Chemischen Industrie im Vergleich zur Gesamtindustrie zwischen 1960 und 1973 eher stabil ist bzw. für eine Reihe chemischer Massenprodukte sogar rückläufige Tendenzen aufweist (Hofmann 1975: 98).

${ }^{33}$ In der Bundesrepublik steigt der Verbrauch an Kunststoffen in Kilogramm pro Kopf der Bevölkerung von 15,0 im Jahr 1960 auf 54,3 im Jahr 1970, der Chemieumsatz in derselben Zeitspanne von 21,8 Mrd. DM auf 43,9 Mrd. DM und die Produktivitätsziffer um 87 Prozent gegenüber dem Ausgangsjahr 1960 (Hofmann 1975).
} 
Rahmen als Massenproduktion statt. Parallel dazu verändern sich Konsumformen und gesellschaftliche Lebensweise und setzen eine „soziale Eigendynamik“ (Wittke 1996) in Gang, die sich im Massenkonsum niederschlägt. ${ }^{34}$ Der Branchenboom der Chemischen Industrie ist auch als Folgeerscheinung einer gesamtgesellschaftlichen Prosperitätsphase, die in Deutschland als „Wirtschaftswunder“ gefeiert wird, zu verstehen. In dieser etwa zwei Jahrzehnte andauernden Phase ist die Chemische Industrie geprägt vom Aufbau großvolumiger und möglichst auf kontinuierlicher Produktion basierender Anlagen zur Herstellung von Industriechemikalien unter Ausnutzung der „economies of scale“ (Chandler 1990). Die starke Nachfrage sowie das Wachstum anderer Branchen forciert diese Entwicklung, gleichzeitig findet durch eine Weiterverarbeitung der Grundchemikalien innerhalb der Konzerne zu veredelteren, konsumnäheren Produkten eine vertikale Vorwärtsintegration statt. Dominant bleibt indes die Produktion petrochemischer Grundstoffe $^{35}$ (ca. 50 Prozent des Gesamtumsatzes), der Anteil des Umsatzes im Bereich der Pharmazeutika spielt mit etwa 10 Prozent eine eher untergeordnete Rolle (Becker 2001). Insgesamt profitiert die Chemische Industrie ebenso wie andere Industriezweige von der fordistischen Nachkriegsprosperität, wobei das Wachstum insbesondere durch die Massenproduktion und den Massenkonsum von Produkten, die aus billiger Erdölenergie herzustellen sind, angetrieben wird. Die Strategie der Diversifizierung der Geschäftsbereiche sichert die Unternehmen vor den negativen Folgen konjunktureller Schwankungen einzelner Produkte ab, da diese durch den Cash flow anderer Bereiche refinanziert werden können.

Anfang der 1970er Jahre steht das Erfolgsmodell des branchentypischen Verwertungskonzepts mit seinen auf der Logik des fordistischen Akkumulationsregimes beruhenden Produkt- und Produktionsstrategien ${ }^{36}$ erstmals zur Disposition. Die sogenannte Ölschocks

\footnotetext{
${ }^{34}$ Dies unterstellt nicht etwa, dass der Gesellschaft der Massenkonsum durch eine dem schlichten ReizReaktions-Schema folgende Logik von Angebot (,billige Massenprodukte werden in der Werbung feilgeboten“) und Nachfrage (,und die Konsumenten kaufen“) oktroyiert wurde. Der Zusammenhang ist wesentlich komplexer, wie Wittke (1996) exemplarisch für die Elektroindustrie zeigt. „Wenn man die Dynamik entschlüsseln will, mit der sich das „Was“ der Produktion verändert hat - und damit der Ausbreitungsdynamik industrieller Massenproduktion auf die Spur kommen möchte - muß man dieser sozialen Eigenlogik neuer Konsumformen nachgehen. Soziale Eigenlogik meint nicht Unabhängigkeit - weder von ökonomischen noch von sozialen, nicht zuletzt gerade durch die Verallgemeinerung von Konsumformen wirksamen Zwängen.“ (vgl. ebd.:70).

${ }^{35}$ Nach dem Zweiten Weltkrieg kann daher in deutschen Großunternehmen auch eine Rückwärtsintegration beobachtet werden, d.h. Mineralölindustrie und Chemische Industrie verschmelzen in Teilen miteinander. Strategisch ging es den chemischen Unternehmen darum, eine kostengünstige und sichere Rohstoffversorgung zu gewährleisten (ausführlich dazu: Becker 2001).

${ }^{36}$ Wie bereits gezeigt, hat sich die deutsche Chemische Industrie innerhalb kürzester Zeit zu einem Kernsektor der Wirtschaft entwickelt, der neben klein- und mittelständischen Unternehmen vor allem von großen Agglomerationen wie in Leverkusen und Ludwigshafen geprägt ist. Hintergrund hierfür ist das für das Wachstum der deutschen Chemie charakteristische Zusammenspiel zwischen Kuppel- und Verbundproduktion (Bathelt 1997). Kuppelproduktion ist ganz allgemein in der Chemischen Industrie ein Prozess, bei dem aus naturgesetzlichtechnischen Gründen zwangsläufig mehr als das Zielprodukt (erwünschtes Kuppelprodukt) hervorgeht. Die Neben- und Abfallprodukte werden als unerwünschte Kuppelprodukte bezeichnet (Riebel 1955). Die Kuppelproduktion wird dann zu einer Verbundproduktion, wenn sich ein Unternehmen entscheidet, möglichst alle Kuppelprodukte, also erwünschte ebenso wie unerwünschte, weiter zu verarbeiten. Äußerlich ist ein Verbundstandort daran zu erkennen, dass die Produktionsbetriebe über ein Rohrleitungssystem miteinander verbunden sind. Über dieses Netz wird der Transfer der Rohstoffe und Kuppelprodukte verbundintern koordiniert. Kuppel- und Verbundproduktion sind die Voraussetzung für die in der deutschen Chemischen Industrie seit dem 19. Jahrhundert zu beobachtende Tendenz zur vertikalen und horizontalen Integration. Dies hat zwangsläufig Auswirkungen auf die räumliche Integration: Ballungsprozesse lassen dominante Industrieagglomerationen entstehen. Das ursprünglich
} 
von 1973 und 1978/79 machen die Abhängigkeit der inzwischen fast vollständig auf Erdöl basierenden Produktion von den Erdöl produzierenden Ländern überdeutlich und führen die bundesdeutsche Industrie in die schwerste Rezession der Nachkriegszeit. ${ }^{37}$ Zwar ist die gesamte Industrie von diesen Entwicklungen betroffen, die chemische Industrie steht jedoch vor einer besonderen Herausforderung, und zwar nicht nur wegen der kurzfristigen Versorgungsengpässe, die es zu bewältigen gilt. Die öffentliche Wahrnehmung der Chemischen Industrie sowohl im Hinblick auf ihre Produkte als auch auf ihre Produktionsbedingungen gerät in die Kritik.

- Unter gesellschaftspolitischen Aspekten setzt die Branche die 1972 veröffentlichte Studie des Club of Rome über die „Grenzen des Wachstums“ unter Druck, in der die baldige Erschöpfung vieler Rohstoffvorräte prognostiziert wird. Die Einsicht, dass fossile Rohstoffvorkommen endlich sind und der Mensch langfristig von einer intakten Natur abhängig sein wird, löst den nahezu uneingeschränkten Fortschrittsglauben der 1950er und 1960er Jahre ab. ${ }^{38}$

- Und schließlich wächst generell die Skepsis der Konsumenten gegenüber chemischen Produkten. Die Risikopotentiale der „Chemisierung des Alltags“ (Beck 1986) rücken zunehmend in den Mittelpunkt der öffentlichen Wahrnehmung und die Chemische Industrie wird zur Zielscheibe der Ökologiedebatte. Neben den spektakulären Katastrophenfällen in Seveso 1976 und Bhopal 1984 sind es vor allem auch die Meldungen über versteckte Umweltgifte im privaten Umfeld wie etwa Asbest, und Dioxin in Baumaterialien, Weichmacher in Kinderspielzeug, Giftstoffe in Außenfarben sowie Berichte über permanente Einleitung von Schadstoffen in Gewässer, die zu einer Sensibilisierung der Öffentlichkeit beitragen.

Diese Entwicklungen geraten zum manifesten und längerfristigen Problem der Branche, und haben erhebliche Konsequenzen für die bis dahin mit „weißer Weste“ agierenden Chemieunternehmen. In groß angelegten Imagekampagnen versuchen die Unternehmen, das Vertrauen der Konsumenten zurück zu gewinnen, doch von erhöhter und kritischer Aufmerksamkeit der Öffentlichkeit kann sich die Chemische Industrie nicht mehr lösen.

„Die kritische Umweltschutzdiskussion hat den vorher weitgehend unangetasteten gesellschaftlichen Grundkonsens darüber aufgebrochen, dass die industrielle Produktion im wesentlichen als Garant von sozialem Fortschritt und gesellschaftlichem Fortschritt anzusehen ist. Dies gilt ganz besonders für die Chemische Industrie.“ (Heine, Mautz 1989: 150ff)

spezialisierte Produktionsprogramm wird durch die daraus resultierenden technologischen, ökonomischen und forschungsbezogenen Synergien immer mehr ausgedehnt (Schumann et al. 1994).

${ }^{37}$ Als erster „Ölschock“ gilt die künstliche Verknappung des Erdöls, mit der die OPEC-Staaten die Ölpreise in die Höhe treiben, um ihren politischen Forderungen an Israel Nachdruck zu verleihen. Die arabischen Staaten des Nahen Ostens drosseln im Oktober 1973 ihre Erdölproduktion, verhängen Lieferboykotte und erhöhen die Preise. Sieben weitere Länder beteiligen sich knapp zehn Tage später an dem Öl-Embargo gegen Länder, die als Verbündete Israels gelten, d.h. vor allem gegen die USA, aber auch gegen die Bundesrepublik Deutschland. Als Auslöser des zweiten „Ölschocks“ gilt der Machtwechsel im Iran 1978/79, in dessen Folge sich die Rohölpreise binnen Jahresfrist verdoppeln.

${ }^{38}$ Hat beispielsweise noch 1979 der weltweite Pro-Kopf-Verbrauch an Energie einen historischen Höchststand, sinkt er innerhalb von vier Jahren, also bis 1983, auf das Niveau von 1975. Autos, Heizungen und Kraftwerke liefen verstärkt mit energiesparenden Techniken, die Wärmedämmung von Gebäuden wird verbessert. Außerdem werden in vielen bundesdeutschen Großstädten alternative Listen und Bürgerbewegungen gegründet, die eine Umweltpolitik jenseits von Ressourcenverschwendung, Raubbau an der Natur und der weiteren Nutzung von Atomkraft anstreben. Die Gründung der Partei „Die Grünen“ im Jahr 1981 trägt zu einer weiteren Mobilisierung und systematischen Ausweitung der Bewegung bei. 
Die Chemische Industrie wird gesellschaftlich in zweifacher Hinsicht in die Pflicht genommen: Sie gilt als Hauptverursacherin eines Großteils ökologischer Probleme und ist gleichermaßen in vielen Bereichen prädestiniert zur Lösung eben dieser Probleme, da häufig die Beseitigung von Schadstoffen auf chemische Vorgänge zurückzuführen ist. Um die Produkte nach wie vor auf den angestammten Märkten absetzen zu können, stellt die Chemie ihre Verfahren und Rezepturen auf neuen Bedürfnisse und Gesetzesanforderungen um, ${ }^{39}$ eine doppelte Kostenbelastung, denn Forschungs- und Entwicklungsaufwand fallen einmal für die Entwicklung der Verfahren und Technologien und für die Umstellung auf diese Verfahren an. ${ }^{40}$

Neben diesen Sicherheits- und Umweltaspekten, die in die Rationalisierungsstrategien mit aufzunehmen sind, sieht sich die Branche auch einem neuartigen und stetig wachsenden Konkurrenzdruck vor allem auf dem Gebiet der Produktion von Grundchemikalien ausgesetzt. Die sich wandelnden Konkurrenzverhältnisse auf dem Weltmarkt stehen im engen Zusammenhang mit der aufholenden Industrialisierung Asiens, Osteuropas und Südamerikas, die als neue Wettbewerber Zutritt zum Weltmarkt finden. Schlüsseltechnologien sowie Rohstoffe zur Produktion von Grundchemikalien sind zunehmend weltweit verfügbar, so dass die Markteintrittsbarrieren deutlich gesenkt werden. Und gerade die Produktion dieser standardisierten Chemieerzeugnisse erfordert kein allzu umfassendes Knowhow, so dass der Nachbau der Anlagen sich relativ unproblematisch gestaltet. Außerdem verfügen die neuen Anbieter außerhalb der OECD Länder über billige Arbeitskräfte und stellen keine hohen Anforderungen an eine umwelt- und ressourcenschonende Herstellung. Innerhalb kürzester Zeit können die chemischen Stoffe damit auf dem Weltmarkt zu niedrigen Preisen bei adäquater Qualität angeboten werden.

Die Unternehmen reagieren auf die durch diese Entwicklungen induzierten Probleme ihrer Kapitalverwertungsstrategie mit organisatorischen und technologischen Modernisierung und Flexibilisierung der Produktion sowie einer ersten umfassenderen Reduzierung von Arbeitskräften. Das Rationalisierungskalkül der deutschen Unternehmen wandelt sich im Zuge dieser Entwicklungen nachhaltig von der Massenproduktion zur diversifizierten Qualitätsproduktion: Die Qualifikation und Know-how betreffende Produktion der Basischemie (Düngemittel, Grundchemikalien, petrochemische Produkte) wird in Länder verlagert, in denen die gesetzlichen Vorschriften niedrig, die Arbeitskräfte billig und die Zielmärkte günstig gelegen sind, verlagert. Am Standort Deutschland wird die Ausweitung der Produktion hochwertiger Güter vorangetrieben. Energie- und Rohstoffverbrauch, Umweltbelastungen und Prozesssicherheit werden - zumindest in den hochindustrialisierten Gesellschaften und so auch in Deutschland - zu zentralen Eckdaten für die Standortentscheidung. Ölkrise und Ökologiedebatte lassen die quantitative Expansion hinter die

\footnotetext{
${ }^{39}$ Die Erfahrungen des Chemieunfalls in Seveso sind ein Grund dafür, dass die zuständigen Institutionen der EG Anfang der 80er Jahre verschärfte rechtliche Anforderungen an die Sicherheit von Industrieanlagen stellen. Mit der Störfall-Verordnung (12. Verordnung zum Bundes-Immissionsschutzgesetz) und der Seveso-Richtlinie der EG werden neue Pflichten für die Betreiber von hochgefährlichen Industrieanlagen erlassen. Wesentliche Elemente sind die Anfertigung von Sicherheitsanalysen, d.h. einer umfassenden Dokumentation der entsprechenden Anlagen und einer systemanalytischen Untersuchung des Anlagenverhaltens bei Störungen, sowie die Verknüpfung der Umsetzung der Sicherheitspflichten mit dem immissionsschutzrechtlichen Genehmigungsverfahren. Maßnahmen werden zunächst staatlicherseits ergriffen: eine umfassendes Umweltschutzgesetz, das deutlich höhere Dokumentations- und sicherheitsanalytische Anforderungen an die Produktion stellt, wird verabschiedet.

${ }^{40}$ So steigt etwa der Anteil der Aufwendungen für den Umweltschutz zwischen 1974 und 1977 von 1.926 auf 2.560 Mrd. DM (bei im Vergleichszeitraum sinkender Investitionsquote).
} 
qualitativen Verbesserungen der Produktionsprozesse zurücktreten. Diese relevanten Neuorientierungen erweisen sich als zukunftsträchtig: Die Umsätze der großen Konzerne bleiben, bei leichten Einbrüchen in den Jahren 1973 und 1978/79, deutlich im positiven Bereich. Die forschungs- und entwicklungsintensiven, veredelten Produkte können mit hoher Exportquote global abgesetzt werden, was zu einer Stabilisierung bzw. sogar leichten Ausweitung der inländischen Beschäftigtenzahlen führt.

„Qualitätsprodukte mit möglichst großem Raffinement zu erzeugen, das scheint das Erfolgsrezept der westdeutschen Chemieindustrie zu sein.“ (Kern, Schumann 1990)

Dafür sprechen auch verkürzte Produktinnovationszyklen (Wöcherl 1989) und gesteigerte Aufwendungen für Forschung und Entwicklung, die sich von 1980 bis 1991 mehr als verdoppeln und deren Quote gemessen am Gesamtumsatz von 4,2 Prozent auf 5,6 Prozent ansteigt. Festzuhalten ist freilich, dass bei aller Spezialisierung auf höherveredelte Produkte die Chemische Industrie in ihrem Kern nach wie vor auf den traditionellen diversifizierten Unternehmensstrukturen mit horizontal integrierten Geschäftsbereichen basiert (Becker 2001).

\subsection{Technisierungsverläufe der Chemieproduktion}

Die Intensivierung und Ausweitung chemischer Produktion von den kleinen Mengen der Laborexperimente zu Chargengrößen von mehreren Tonnen ist historisch ohne die Entwicklung und Einführung spezieller Verfahrenstechniken nicht denkbar. Charakteristisch für die Produktion chemischer Stoffe ist, dass verschiedene Aggregatzustände durchlaufen werden, in denen eine Ursprungssubstanz modifiziert wird. Synthesen, Auf- und Abspaltung sowie Umgruppierung molekularer Strukturen bilden die Grundlagen der Produktherstellung und sind eigenständige Prozesse, die auf der gegebenen Interaktivität chemischer Elemente beruhen. Die im Labor angewandten Verfahren geben allerdings nur ein erstes Synthesemodell vor, aus dem prinzipiell das Endprodukt hergestellt werden kann. Die Weiterentwicklung zu einem industriellen, wirtschaftlichen Verfahren im benötigten Produktionsmaßstab, das so genannte Scaling up, stellt die Forschungs- und Entwicklungsabteilungen in der chemischen Industrie vor allem vor die Herausforderung, teure durch billige Vorprodukte zu substituieren, den Energiebedarf sowie die Anzahl der Reaktionsstufen möglichst gering zu halten, Abfälle zu vermeiden und die Ausbeute zu optimieren. Im historischen Verlauf kennzeichnen drei Etappen diesen technischen Rationalisierungsprozess.

\subsubsection{Produktion im Großküchenstil}

Die industrielle „Massen“ - Produktion der Chemischen Industrie ist vor 1900 eng an Laborexperimenten ähnelnde Verfahren gebunden. Die Produktionsmittel sind äußerst einfache Abbildungen der Laborgeräte, und leicht zu bedienende Kessel, Kübel und Pfannen gehören zur Grundausstattung der ersten Fabriken. Die chemischen Reaktionen laufen der Reihe nach in einem diskontinuierlichen und wenig störanfälligen Prozess ab. Ist eine sogenannte Charge produziert, werden die Behältnisse gereinigt und der nächste Ansatz 
aufgesetzt, ein mühsames und bezogen auf die Produktausbeute der ersten „Fabriken““41 wenig effektives Geschäft. Zwar sind die leichte Handhabung der Geräte, geringe Schadens- und Störempfindlichkeit wie auch die Vielfalt im Hinblick auf die Einsatzmöglichkeiten der Gerätschaften zunächst vor Vorteil, ihnen stehen aber erhebliche Nachteile gegenüber:

- Die Bedienung sowie der Transport der Vor- und Endprodukte bindet viele Arbeitskräfte;

- Die belastenden Arbeitsbedingungen führen zu Rekrutierungsproblemen;

- Die Produkte unterliegen hohen Qualitätsschwankungen.(Kern, Schumann 1970:158)

Insbesondere die geringen Produktmengen stellen für die Unternehmen ein Problem mit hohem Stellenwert dar. Ist ein innovatives Produkt erst einmal auf den Markt gebracht, so sichert die Monopolstellung dem first mover zwar seine Gewinnmarge, mittelfristig hingegen ist damit zu rechnen, dass ein Konkurrenzprodukt auf den Markt kommt und die Preise sinken. Im allgemeinen Gründerboom der Chemischen Industrie ist dieser Druck erheblich, und so sichert nur die schnelle und massenhafte Produktion die Gewinne.

\subsubsection{Mit der Verfahrenstechnik zur Massenproduktion}

Der verfahrenstechnisch ausgereifte Durchbruch zur industriellen Massenproduktion gelingt den Unternehmen um 1900 und wird von der industriellen Anwendung des sogenannte Haber-Bosch-Verfahrens initiiert. Bosch war als Industriechemiker die Übersetzung der von Haber entwickelten Ammoniak-Synthese vom Labormaßstab in die Großtechnik gelungen. Dieses innovative Verfahren ermöglichte erstmals eine schnelle, effiziente Produktion großer Mengen chemischer Stoffe. Neben dieser Erfindung mit Initialeffekt ist die technische Rationalisierung in der Branche von zwei Grundprinzipien getragen (Schumann et al.1994):

Vergrößerung der Anlagen. Der Rationalisierungseffekt besteht darin, dass die Mehrkosten für eine technisch gleiche, aber größere Anlage auf die Materialkosten für die Oberfläche beschränkt bleiben, während die Durchsatzmenge proportional zum Volumen wächst. Darüber hinaus sinken die Energieverluste, denn auch sie sind oberflächen - und nicht volumenabhängig. Die Kosten pro Produktionseinheit werden so reduziert.

Kontinuisierung von Prozessen. Wo es das Verfahren erlaubt, löst die kontinuierliche die diskontinuierliche Produktion ab. Der Produktionsprozess wird dabei nicht mehr durch Vorbereitung, Entleerung, Beschickung und Transport unterbrochen, sondern findet in einem mechanisch gesteuerten Aggregatsystem statt, welches alle Reaktionen miteinander verbindet. Der Rationalisierungseffekt ergibt sich aus der fortlaufenden Nutzung der Apparate, Produktionsstillstände werden so vermieden und die Prozesse können stabiler ablaufen . Diese Kontinuisierung kann auf einzelne Aggregate beschränkt bleiben, oder je nach Komplexität der Reaktion auf mehrere Stufen der Stoffumwandlung ausgedehnt werden (Kern, Schumann 1985: 158f).

\footnotetext{
${ }^{41}$ Zum Vergleich: 1866 ist die mit Fabrikgebäuden überbaute Fläche der BASF 55 Ar groß, 1888 bereits 1473 und 19144370 Ar (Christ 1995).
} 


\subsubsection{Automatisierung und Digitalisierung}

Der Übergang von mechanischen zu automatisierten Systemen beginnt in Deutschland im ersten Drittel des 20. Jahrhunderts. Mit der Vergrößerung der Anlagen und der Durchsatzmengen sowie dem wachsenden Anteil an kontinuierlichen Prozessen zeichnen sich die Grenzen der Beherrschbarkeit mechanischer Kopplung ab. Die Kontinuisierung der chemisch-verfahrenstechnischen Prozessabläufe führt zu unkalkulierbaren Rückkopplungen zwischen nicht zu trennenden Teilprozessen. Unter den gegebenen Umständen sind diese Reaktionen von den Arbeitern kaum noch steuer- und kontrollierbar. Die technische Lösung dieses neuartigen Beherrschungsproblems gelingt mit dem Einsatz kybernetischer Regelinstrumente, wie etwa Thermostaten (Hirschhorn 1984; Tacke 1997). Diese Regelinstrumente kompensieren die Abweichungen der temporär instabilen Prozessabläufe selbsttätig, d.h. unabhängig von menschlichen Eingriffen.

„Der Wechsel im Technisierungskonzept, der vom mechanischen Ablauf zum automatischen Regelkreisprinzip führt, beruht auf der Ausdifferenzierung einer eigenständigen technischen Steuerungskomponente, durch die ein mechanisches System, auf das sie reflexiv angewandt wird, einen bisher nicht erreichten Grad an Autonomie gegenüber notwendiger Steuerungsarbeit erhält.“ (Tacke 1997:147)

Beschränkt sich Automatisierung in der Frühphase auf lokale Regler, setzen sich Anfang der 1950er Jahre zentrale technisch integrierte Steuerungseinheiten durch, und seit Mitte der 1960er Jahre sind die Prozesse in der Großchemie gekennzeichnet durch eine „Technik der teilautomatisierten Großanlage“.

Diese Technologie stellt die technische Einlösung eines auf spezifischen Branchenbedingungen (säkulare Wachstumsindustrie mit großen ökonomischen Ressourcen, die in mechanisierungs- und automatisierungsfreundlichen Prozessen durch chemische Umwandlung bzw. physikalische Bearbeitung Massenprodukte herstellt) aufbauenden Verwertungskonzepts dar. (Kern, Schumann 1990:235)

Verschiedene Prozessfunktionen werden in großvolumigen Anlagen hintereinander geschaltet und in miteinander verschränkten Stufen zu einem komplexen technischen System integriert. Alle relevanten Prozessgrößen werden automatisch gemessen und über Regler geführt. Die flüssigen, gasförmigen oder kristallinen Stoffe sind dabei in einem kontinuierlichen Fluss, und mittels automatischer Steuerung können Endprodukte in großen Mengen hergestellt werden.

Die fortschreitende Automatisierung stellt die Industrie vor ein neues Problem: Die Anlagen werden immer schneller, komplexer und damit risikoanfälliger, ein neues „Niveau der Unberechenbarkeit“ (Tacke 1997:145) wird erreicht. Überraschende Abweichungen vom Normalbetrieb sind nicht nur wahrscheinlicher, sondern zudem in Anbetracht der immer größeren Stoffmengen immer gefährlicher. Gleichzeitig entzieht die Verkettung der Prozesse die einzelnen Reaktionen mehr und mehr der Kontrolle durch die Arbeiter.

"Traditional machinery transforms by constraining movement until only perfect movement is realized. The resulting sequence is invariant. In contrast, the feedback loop transforms by importing error and developing a sequence of continually compensation movements so that a fixed outcome is achieved. The resulting sequence is flexible, changing as input conditions change.” (Hirschhorn 1984:28) 
Den naheliegenden Schritt zur Vollautomatisierung durch rechnergestützte Regelsysteme geht die Chemische Industrie allerdings vorerst nicht, bis in die 1970er Jahre hinein überwiegt die pneumatische Regelungstechnik. Sie hat sich international als Standard durchgesetzt, gilt als technisch ausgereifte und relativ billige Technik, die von den Beschäftigten ohne Probleme bedient werden kann (Kern, Schumann 1990:236). Der hohe Umsetzungsaufwand, eine noch mangelhafte Messwerterfassung, die Abhängigkeit vom Zentralrechner sowie Schwierigkeiten mit der Mathematisierung der Prozesse machen die neue Technologie demgegenüber zu einer teuren und riskanten Alternative .

Auf der technischen Ebene setzen grundlegendere Veränderungen in Form des Einsatzes digitaler Prozessleittechnik (PLT) erst Ende der 1980er Jahre ein. Die Leistungsfähigkeit und Bedienungsfreundlichkeit der Systeme verbessert sich kontinuierlich. Außerdem zeigt sich, dass die konventionelle Mess- und Regeltechnik den sich wandelnden Marktund Produktionsbedingungen zunehmend weniger gerecht wird, und eine diversifizierte Qualitätsproduktion eher mit den digitalen Techniken betrieben werden kann. Investitionen in vollautomatisierte Prozesstechnologie zahlen sich aber auch deshalb aus, weil sie als weiteren Effekt die Koordination der Produktionsaktivitäten auf Feld-, Prozess-, Betriebs- und Unternehmensleitebene erleichtern (Bathelt 1997). Zusammenfassen sprechen vier Argumente für den verstärkten Einsatz der PLT (Schumann et al. 1994): Die Reduzierung des Aufwands bei An- und Abfahrvorgängen, der erweiterte und differenzierte Informationszugriff sowie verbesserte Dokumentationsmöglichkeiten, die gerade im Zusammenhang mit der Umweltdebatte an Gewicht gewinnen, die insgesamt besseren Möglichkeiten der Feinsteuerung und Optimierung der Prozesse und schließlich die besseren Chancen zur Vermeidung von Qualitätsschwankungen. Zu einer flächendeckenden Ablösung der konventionellen Mess- und Regeltechnik durch die digitale PLT kommt es aus produktionstechnischen Überlegungen indes nicht. Der Austausch der alten gegen die neue Technologie rechnet sich nicht in den Bereichen, in denen Produktionsmodernisierung nicht verbunden ist mit dem Aufbau neuer Anlagen oder einer erheblichen Kapazitätserweiterung (Produktpalette oder Produktmenge). Und einige Produktionsanlagen entziehen sich aufgrund ihrer Produktpalette einer solchen technischen Rationalisierung, da sie Produkte herstellen, die im Labormaßstab produziert werden und bei denen schon ein übermäßiger Einsatz von Mess- und Regelinstrumenten kaum Sinn macht. (Schumann et al. 1994) Bei allen Einschränkungen steht jedoch fest, dass die Phase des Ausprobierens und Abwägens zwischen digitaler Prozessleittechnik und konventioneller MSR Technik in der Chemischen Industrie vorbei ist und „der Zug- wenn auch im gemäßigten Tempo - in Richtung digitaler Prozessleitsysteme fährt.“ (Schumann et al. 1994: 555).

\subsection{Die Organisation von Innovation}

Sowohl im Bereich der Produkt- wie der Verfahrensinnovationen ist die Chemische Industrie Deutschlands seit ihrem Take-off äußerst erfolgreich und erlebt eine nahezu uneingeschränkte Phase der Prosperität. Entscheidend für diese konjunkturrobuste Entwicklung ist ein erfolgreiches Innovationsmodell, das durch eine effektive Nutzung wissenschaftlicher Erkenntnisse für innovatorische Prozesse gekennzeichnet ist. Dieses wissenschaftliche Wissen wird nicht nur aus den Bereichen der akademischen Wissensproduktion in die Industrie transferiert, sondern umgekehrt gibt es auch Transferströme von 
Know-how etwa in Form personeller Fluktuation aus der Industrie in die Wissenschaft. ${ }^{42}$ Grundlagenforschung, angewandte Forschung und experimentelle Entwicklung ${ }^{43}$ werden in diesem Modell eng verknüpft, und es kommt zu Synergieeffekten sowohl im Hinblick auf die Generierung von Forschungs- wie auch von Ausbildungswissen (König 1995).

\subsubsection{Chemie als neue Wissenschaft - Chemische Industrie als neue Industrie}

Entscheidend für diese Entwicklung ist zunächst ein spezifisches und sich seit der Renaissance herausbildendes Wissenschaftsverständnis, denn die Hervorbringung neuen Wissens und die Generierung von Innovationen entspricht zunächst nicht dem Selbstverständnis des „klassischen“ Gelehrten. ${ }^{44}$ In den seit dem 12. Jahrhundert in ganz Europa gegründeten Universitäten beschränkte man sich auf die Lehre, d.h. auf die Vermittlung von Wissen. Die Positionen und Interpretationen der großen Philosophen der Vergangenheit galten als unumstößlich und unersetzlich, d.h. ihre Erkenntnisse und Theorien bildeten den Dogmen-Kanon, den es zu beherrschen galt. Eine weitaus größere Rolle für die Generierung neuer Produktideen als die Wissenschaft spielte handwerkliches Können, Tradition und Überlieferung, und es existiert bis ins 19. Jahrhundert hinein eine Pluralität vorwiegend nicht-kodifizierten Wissens im Bereich der mittelalterlichen Handwerksinstitutionen. In dieser Sphäre wurde durch Zufallsentdeckungen oder die Umkehrung von Fehlschlägen ein Großteil neuer Produkte und Verfahren entwickelt. Ein Transfer zwischen den beiden „Wissenspolen“ wurde weder betrieben, noch interessierte sich der akademische Zirkel sonderlich für das Handwerk (Burke 2001).

\footnotetext{
${ }^{42}$ Die folgende Darstellung ist insbesondere inspiriert durch die Veröffentlichungen von Hack, Hack (1985) und König (1995), die sich u.a. mit der ,Verwissenschaftlichung' der Chemischen Industrie befassen, sowie von Hack (1998) und Weingart (2001), die sich mit Wissensformen und Organisationsstrukturen allgemein sowie u.a. der Elektroindustrie befassen.

${ }^{43}$ Im allgemeinen Sprachgebrauch wird unter Grundlagenforschung zumeist die Entwicklung naturwissenschaftlicher Theorien allgemeiner Art verstanden, bei der spezielle Anwendungs- bzw. Nutzungsformen kommerzieller Art keine Rolle spielen („Nicht-anwendungsbezogen“ ist auch diese Forschung in den seltensten Fällen, so verweist etwa Drews (1998) im Hinblick auf die pharmazeutische Forschung, deren Ziel, die Heilung oder Linderung von Krankheiten, ist sehr wohl ein außerwissenschaftliches, aber eben kein primär ökonomisches). Diese Form der Forschung gilt als „zweckfrei“. Industrielle Grundlagenforschung hingegen ist auf Nahziele ausgerichtet und geplantes Forschen zur wirtschaftlichen Nutzbarmachung der gewonnenen wissenschaftlichen Erkenntnisse. Angewandte Forschung ist ausschließlich auf die Gewinnung neuer wissenschaftlicher oder technischer Erkenntnisse gerichtet und dabei auf eine fest umrissenen praktische Anwendung fixiert, um neue oder verbesserte Verfahren zu ermitteln. Experimentelle Entwicklung ist die systematische Anwendung und Entwicklung von Forschungsergebnissen mit dem Ziel, zu neuen oder wesentlich verbesserten Werkstoffen, Geräten, Produktionsverfahren oder Dienstleistungen zu gelangen. Indes gilt: Nützliches Wissen wird auch im universitären Kontext generiert, Grundlagenwissen auch in Industrielaboratorien. Von einem linearen Modell des Wissenstransfers muss daher Abstand genommen werden und im Anschluss an Etzkowitz, Webster und Healey (1998) wird auch dieser Arbeit ein iteratives bzw. rekursives Modell des Wissenstransfers zugrunde gelegt (vgl. Weingart 2001). Als wesentliches Merkmal zur Unterscheidung zwischen akademischer und industrieller Forschung gilt uns einmal die Kontrolle über die Forschungsziele. Während diese im akademischen Bereich durch die Wissenschaft selbst kontrolliert werden, wird industrielle wissenschaftliche Forschung von der Wirtschaft kontrolliert. Darüber hinaus unterscheidet sich die Interpretation von Ergebnissen erheblich: Wissenschaftliche Forschung kann per definitionem ein Ziel nicht verfehlen, denn auch das Nicht-Erreichen eines Ziels gilt als positiv konnotierte Wissensgenerierung. Negative Ergebnisse in der industriellen Forschung hingegen werden vor allem als Verluste von Forschungs- und Entwicklungskosten interpretiert. Unter gesellschaftlichen Aspekten ist dies insofern problematisch, als industrielles Wissen aus patentrechtlichen Gründen nicht veröffentlicht wird. Wissensbestände, die nicht die gewünschten aber eben doch „Ergebnisse“ im wissenschaftlichen Sinn bedeuten, ruhen damit „auf Halde“, und können nicht im Wissenschaftssystem genutzt werden.

${ }^{44}$ Wir konzentrieren uns im folgenden auf die Entwicklung in Europa.
} 
Erst im Zug der Renaissance entwickelte sich ausgehend von den mehrheitlich in Italien gegründeten Akademien ein „neues Denken“, dass sich bewusst kritisch mit dem universitären Kanon auseinander setzte. Die Naturphilosophie bzw. mechanische Philosophie brachte die Abkehr von klassischen und mittelalterlichen Traditionen, eine Bewegung, die als „wissenschaftliche Revolution“ bezeichnet wird und den ersten Brückenschlag zwischen Erfahrungswissen und wissenschaftlichem Wissen versucht. ${ }^{45}$

„Ebenso wie die Humanisten, jedoch in größerem Maßstab, versuchten die Anhänger dieser Bewegung, alternatives Wissen in die Gelehrsamkeit einfließen zu lassen; die Chemie etwa entwickelte sich zu einem beträchtlichen Teil aus der handwerklichen Tradition des Hüttenwesens,...“ (Burke 2001:53)

Nachvollziehbarkeit und Prüfbarkeit werden zu fundamentalen Kriterien von Wissenschaft, und das Primat der Rationalität löst das auf Gottesvorstellungen basierende Weltbild ab. Konkrete physikalisch - chemische Reaktionen stehen im Mittelpunkt des Forschungsinteresses, ersetzen ganzheitliche Betrachtungsweisen und bilden den Rahmen für den Aufschwung, den die beiden Erfahrungswissenschaften Chemie und Physik erleben. Im ausgehenden 18. Jahrhundert zeichnet sich schließlich das Ende der Ära der Universalgelehrten ab, und die Spezialisierung und Ausdifferenzierung der Wissenschaften an den Universitäten beginnt. In Deutschland verbindet sich diese Entwicklung mit einem am Humboldtschen Ideal orientierten Selbstverständnis der Professoren, das darauf zielt, nicht nur für die Wissenschaft zu arbeiten, sondern auch zusätzliches Wissen zu generieren (Burchardt 1992).

Die Entwicklung der ersten Chemieunternehmen verläuft parallel zur chemischen Wissenschaft, und der Einfluss der „neuen“ qualitativen und quantitativen Forschungsmethoden auf existierende Techniken und Verfahren bleibt gering. „Trial and error“ dominieren innerhalb der Fabriken, und die Entwicklung unter der Anleitung eines erfahrenen Praktikers gilt als vielversprechender und kostengünstigster Weg zu neuen Produkten, Verfahren und Techniken (Burchardt 1979). In der ersten Expansionsphase der Chemischen Industrie dominiert eindeutig handwerklich vorgeprägtes Erfahrungswissen und es wäre falsch davon auszugehen, dass die chemische Industrie zur Zeit ihres Wachstums auf einer chemischen Wissenschaft basierte. Fortschritt und Innovationen fußten auf technischem Know-How, nicht auf wissenschaftlichen Methoden (Wetzel 1991). Professoren wie Studierende stammen zu Beginn des 19. Jahrhunderts mehrheitlich aus dem Bildungsbürgertum und halten sich bis in die 1850er Jahre der gewerblichen Wirtschaft fern - der sie zu dem Zeitpunkt allerdings auch kaum eigene Theorien und Modelle anzubieten haben. $^{46}$

\footnotetext{
${ }^{45}$ Zur Kritik an diesem Begriff vgl. Shapin (1996).

${ }^{46}$ Zwei Ausnahmen mit weitreichenden Folgen bestätigen diesen Zusammenhang zwischen Wissenschaft und Wirtschaft: 1775 setzt die Pariser Akademie einen Preis für ein Verfahren zur Produktion von Soda aus Kochsalz aus. Leblanc gelingt dies, seit 1791 steht das „Leblanc-Soda“ in praktisch unbegrenzter, reinerer und billigerer Form zur Verfügung als die Natursoda. Erstmals wurde gezielt zur Sicherung der Nachfrage auf einem expandierenden Markt für Textilien im weitesten Sinn wissenschaftliches Know-how eingefordert. Die Produktion künstlicher Farbstoffe ist ab 1834 mit der Entdeckung des Farbstoffs Anilin durch Runge möglich. Dennoch dauert es etwa zwei Jahrzehnte, bis ein ganz anderer Farbstoff eine Rolle in der industriellen Produktion spielt. Nach wie vor dominieren Naturfarbenlieferanten den Markt, und erst um 1856 geht diese Ära zuende: In England wird auf
} 


\subsubsection{Forschungsergebnisse und industrielle Verwertbarkeit}

Erst die Weiterentwicklung, Professionalisierung und Autonomisierung der Chemie innerhalb des wissenschaftlichen Fächerkanons wie auch die Formulierung der Strukturtheorie für organische Verbindungen durch Kekulé im Jahr 1865 forcieren schließlich die Zusammenarbeit von Industrie und Wissenschaft. Denn mit der Rekonstruktion des Benzolmoleküls entdeckte Kekulé mit der organischen Chemie nicht nur ein völlig neues Forschungsgebiet, sondern legte auch den Grundstein für die Verzahnung von Wissenschaft und Technik, die die Epoche des „modernen Wirtschaftswachstums“ eröffnet. ${ }^{47}$ Ergebnisse empirischer Experimente können theoretisch erklärt und hergeleitet werden, was nicht nur zu einem sprunghaften Anstieg des Wissens um chemische Prozesse führt, sondern eine Vielzahl von Anwendungsmöglichkeiten insbesondere im Bereich der Farbstoffe erschließt. Theoretisch-chemisches Wissens wird somit attraktiv für Wirtschaftsunternehmen, vor allem die seit den 1860er Jahren expandierende Farbenchemie ${ }^{48}$ ist an den neuen Forschungsergebnissen interessiert, um ihre Produktpalette zu erweitern und ein neues Farbenspektrum abdecken zu können. Wissenschaftliches Know-How wird neben Geld und technischer Perfektion zur wichtigen Ressource.

Zunächst agieren die Unternehmen passiv und eher risikoavers. Sie investieren in klar abgegrenzte und wissenschaftlich sorgfältig recherchierte Produktionszweige. Die Kenntnis eines beliebigen Synthesewegs in der Laboratoriumsapparatur reicht nicht aus, sowohl der industrielle Bedarf als auch die Potentiale zur Umsetzung in ein wirtschaftliches, funktionierendes technisches Verfahren müssen vor einer Investition gesichert sein. Ist dies gewährleistet, zeigen sich die Unternehmen allerdings äußerst „großzügig“: Sie stellen den wissenschaftlichen Labors Arbeits- und finanzielle Hilfen in großem Umfang zur Verfügung. Als Gegenleistung fordern sie von den beteiligten Wissenschaftlern nicht nur die Forschungsergebnisse, sondern ein hohes Maß an Loyalität. Die Professoren verpflichten sich zur Zurückhaltung von Forschungsergebnissen und gehen zumeist exklusive Beziehungen mit einem Industrieunternehmen ein. Die Studierenden bearbeiten neben rein wissenschaftlich orientierten Arbeiten zunehmend praxisnahe und von den Unternehmen vermittelt durch die Professoren gezielt gesteuerte Forschungsthemen (Wetzel 1991). Insgesamt gilt für diese Phase der Internalisierung der Wissenschaft, dass an

„die Stelle spontaner Neuerungen, die indirekt von den gesellschaftlichen Produktionsprozessen heraufbeschworen worden waren, [...] der geplante Fortschritt von Technologie und Produktgestaltung“ trat. Wissenschaft selbst wird zur Ware, die wie die anderen Instrumente und Arbeitskräfte der Produktion gekauft und verkauft werden kann.“ (Bravermann 1977:131)

Insbesondere in Deutschland entwickelt sich eine ebenso spezifische wie effektive Kombination aus Begabungspool, unternehmerischer Initiative und forschungsorientierter

der Suche nach einem Verfahren zur Herstellung von Chinin von Perkin der erste Teerfarbstoff Mauvein entdeckt und eine Anlage zur Fabrikation des blauen Farbstoffs aufgebaut.

${ }^{47}$ S. Kuznets zitiert nach Landes (1999).

${ }^{48}$ Initialzündung für den Aufschwung zur Herstellung von auf synthetischen anstelle natürlichen Farbstoffen basierenden Farben ist die Weltausstellung in London 1862, bei der erstmals Möglichkeiten zur Anwendung synthetischer Farben präsentiert und euphorisch aufgenommen werden (Verg 1988). 
Wissenschaftskultur (Landes 1999), und eine erste, noch äußerst rudimentär ausgeprägte Verwissenschaftlichung der Produktion beginnt. Anzahl und Stellenwert der industrieeigenen Forschungslaboratorien steigen seit 1880 stetig an, und insgesamt kommt es zu einer für beide Seiten idealen Form der Arbeitsteilung innerhalb der Innovationskette: In punkto Basisinnovationen liegt die Kernkompetenz der Akademiker innerhalb der Hochschulen in der Entdeckung und Formulierung allgemeiner Theorien und der Entwicklung abstrakter Verfahrensprinzipien, die zu einem Großteil von den Unternehmen finanziert werden. Die Industrie hat privilegierte Zugriffsrechte auf dieses Wissen, in den Forschungslaboratorien kann die Arbeit auf das Gebiet inkrementeller Innovationen, also der Weiterentwicklung und Optimierung von etablierten Stoffen sowie effektiverer, großtechnischer Verfahren fokussiert werden.

Allerdings arbeitet nach wie vor nur eine Minderheit der von den Hochschulen kommenden Chemiker direkt und ohne Rückbindung an die Hochschule in der chemischen Industrie. Das Anforderungsprofil der Industriechemiker orientiert sich bis in die 1870er Jahre hauptsächlich an der Produktion, ein für die Forscher recht unattraktiver, nichtakademischer Aufgabenbereich. ${ }^{49}$ Denn: Noch zielen die Unternehmen bei der Einstellung von Hochschulabsolventen nicht auf das spezialisierte Wissen, das diese bei ihren Diplom- oder Doktorarbeiten erworben haben. Vielmehr schätzen sie zum einen das umfangreiche Repertoire an handwerklich-methodischem Umgang mit Stoffen und Verfahren, dass an den Universitäten gelehrt wird. Die Chemiker sind durch ihre Ausbildung in methodisch systematischen Vorgehensweisen geschult und besitzen eine abstrakte Problemlösungskompetenz. Zum anderes wird aber auch ihre soziale Kompetenz zugerechnet in den personell rasch expandierenden Betrieben zunehmend von Bedeutung (König 1995).

\subsubsection{Wissenstransfer zwischen Universität und Industrie}

Erst mit dem deutschen Patentgesetz von $1877,{ }^{50}$ welches Produktionsverfahren, nicht aber neue Produkte schützt, verstärkt sich die Nachfrage nach Akademikern auch innerhalb der Industriebetriebe. Die in ihrer Frühphase als „Nachahmungsindustrie“ zu charakterisierende Branche gerät nun unter Druck, eigene Forschung zu betreiben, um der Konkurrenz mögliche alternative Verfahren zur Produktion eines neuen Stoffs durch Sperrpatente zu verbauen. Gleichzeitig versucht die Konkurrenz mit der Patentlegung durch ein anderes Unternehmen, ihrerseits den neuen erfolgreichen Stoff auf einem anderen mögli-

\footnotetext{
${ }^{49}$ Erfahrungsberichte und Briefe von Chemikern aus dieser Zeit belegen, dass die Arbeitsbedingungen in den ersten Chemiefabriken nicht nur unter wissenschaftlichen Aspekten wenig attraktiv waren, sondern die Laboratorien auch im Hinblick auf Ausstattung und Sauberkeit zunächst weit hinter den aus den Universitäten gewohnten Standards zurückblieben. „Das Laboratorium befand sich am Ende der Fabrik im Parterre eines alten Wohnhauses. Ein gelinder Schreck überfiel mich, als ich dieses betrat. Ich war die schönen Laboratorien der Universitäten Leipzig und Zürich gewohnt und hatte mich der Hoffnung hingegeben, auch hier ein nettes Laboratorium vorzufinden. Stattdessen betrat ich geradezu schmutzige Räume. (...)“(Arnold Fischer, Chemiker bei Bayer, Eindrücke seiner ersten Betriebsbesichtigung um 1880, zitiert nach Peetz (1981:51).

${ }^{50}$ Erfinder sollen als Belohnung ein vorübergehendes Monopol für die gewerbliche Nutzung ihrer Erfindung erhalten, wenn sie es als erste beim Patentamt zum Schutz angemeldet haben. Es soll vor allem verhindert werden, dass Erfinder durch Geheimhalten ihrer Erfindung den Gewerbefleiß und die technische Entwicklung bremsen. Der Wettbewerber soll angeregt werden, durch das Suchen nach Umgehungslösungen und Weiterentwicklungen den technischen Fortschritt zu fördern.
} 
cherweise wirtschaftlicheren Weg herzustellen. ${ }^{51}$ Wirtschaftlichkeit definiert sich nicht mehr ausschließlich über die Entdeckung und Vermarktung neuer Stoffe, sondern auch über den Einsatz innovativer Verfahren (König 1995). Die Unternehmen verstärken ihre Forschungstätigkeiten auf drei Ebenen: Innerhalb der Betriebe werden Betriebslaboratorien eingerichtet, die sich vor allem auf Verfahrensverbesserungen spezialisieren und die das praxis- und erfahrungsbasierte Vor - Ort - Wissen der Produktion nutzen. In dezentralen anwendungstechnischen Laboratorien werden wissenschaftliche Forschungsergebnisse verfahrenstechnisch umgesetzt und alternative Herstellungsmöglichkeiten gesucht. In den zentralen Forschungslaboratorien schließlich wird Grundlagenforschung für die unterschiedlichen Produktzweige des Unternehmens betrieben. ${ }^{52}$ Parallele oder sich überschneidende Forschungsaktivitäten werden durch die Einrichtung dieser „Zentral“- oder „Hauptlabors“ vermieden, da in ihnen ebenfalls ein Stab von Wissenschaftlern zusammenarbeitet und die „Forschungsfäden“ zusammenhält.

Für die wissenschaftliche Forschung an den Universitäten entsteht so ein attraktives Betätigungsfeld, und die wachsende Anzahl von Absolventen findet in den meist besser als die universitären Einrichtungen ausgestatteten Laboratorien ${ }^{53}$ der Industrie ein neues Beschäftigungsfeld. Und auch die Professoren, die an den Hochschulen bleiben, profitieren, indem sie lukrative Nebentätigkeiten als Gutachter oder in Aufsichtsräten übernehmen. Darüber hinaus sichern die Unternehmen in einigen Fällen die Finanzierung der Grundausstattung von Laboratorien oder vergeben gut dotierte Forschungsaufträge an Hochschullehrer. ${ }^{54}$ Die Chemiefirmen bemühen sich auf diesem Weg, Professoren an sich zu binden, um den exklusiven Zugang zu Forschungsergebnissen zu sichern sowie die Begutachtungsprozesse neuer Produkten zu beeinflussen. (König 1995).

\subsubsection{Zunehmende Autonomie der Industrieforschung}

Der Beginn des 20. Jahrhunderts steht für die immer stärker werdende Autonomie der Industrieforschung im Verhältnis zu den Hochschuleinrichtungen. Dies ist insbesondere als Reaktion auf das immer komplexere chemischen Wissen ${ }^{55}$ und die Ausweitung der Produktpalette zu verstehen. Die (Er-)Forschung und Entwicklung von Pharmazeutika,

\footnotetext{
${ }^{51}$ Interessant ist in diesem Zusammenhang, dass sich die first mover der Chemischen Industrie mit ihrem sicheren Zugang zu akademischem Wissen für die weitere Expansion wesentliche Claims gesichert haben, und es in der Folgezeit für neue Start-up bedeutend schwerer sein wird, einen Marktzugang zu finden. Stehr (2001) sieht in einem solchen Vorgang eine Analogie zu dem von Robert K. Merton für das Wissenschaftssystem konzipierte Matthäus-Prinzip (Merton 1985). Wer hat, dem wird gegeben, bedeutet für den vorliegenden Fall: Die Vorteile zusätzlichen akademischen Wissens gehen mit großer Wahrscheinlichkeit an diejenigen Unternehmen, die schon auf Grund vorangegangener Erfindungen und Entdeckungen Vorteile genießen.

${ }^{52}$ Die Produktpalette der ersten integrierten Unternehmen besteht zunächst aus Farben und Pharmazeutika, um 1890 beginnt der Einstieg in die Produktion anorganischer Stoffe (v.a. Schwefel-, Salpeter-, Salzsäure), später dann Kautschuk, Kunststoffe, Düngemittel, Lacke etc.

${ }^{53}$ So haben die Unternehmen neben den besseren technischen Geräten darüber hinaus den Vorteil, dass sie Stoffe in hoher Reinheit nahezu unbegrenzt für die Laborarbeit zur Verfügung stellen können.

${ }^{54}$ Im Rahmen eines solchen Vertrags entwickelt etwa Fritz Haber an der TH Karlsruhe seine Laborlösung der Ammoniaksynthese für die BASF.

${ }^{55}$ Die chemische Wissenschaft kann im Bereich der organischen Chemie zwischen 1800 und 1910 eine überproportionale Zunahme von Wissen und Entdeckungen verzeichnen. Die Anzahl der bekannten organischen Verbindungen wächst von ca. 500 (1800) auf etwa 3.000 im Jahr 1860, 15.000 im Jahr 1880 und schließlich auf 150.000 im ersten Jahrzehnt des 20. Jahrhunderts (Burchardt 1992).
} 
Düngemitteln und vielfältigen Zwischen- und Endprodukten erfordert einen immer höheren finanziellen und personellen Aufwand, zugleich verstärkt sich der Markt- und Konkurrenzdruck. Die aus dieser komplizierten Gemengelage entstehenden qualitativen wie quantitativen Anforderungen erfordern die effektive Kooperation von Spezialisten unterschiedlichster Kompetenzen und sind darüber hinaus immer anwendungsorientierter. Die Forscher in den Industrielaboren verfügen in diesem Bereich über einen doppelten Vorteil: Sie haben infolge des wirtschaftlichen Wachstums genügend Kapital und produzieren die zur Forschung benötigten Stoffe selbst. Zudem profitieren sie von der räumlichen Nähe der Laboratorien untereinander wie auch dem direkten Zugriff auf die Produktion. Synergetische Effekte ${ }^{56}$ zwischen den unterschiedlichen Forschungs- wie Produktionsbetrieben werden immer stärker ökonomisch genutzt. Die stetige Professionalisierung der industriellen Eigenforschung sowie die damit verbundenen erheblich höheren finanziellen Aufwendungen verschärfen die Kriterien der Mittelvergabe der Unternehmen an die Hochschulen, immer stärker rückt der konkrete Anwendungsbezug in den Vordergrund. Zunehmend werden speziell die Projekte unterstützt, die anschlussfähig an das firmeninterne Know-how sind und deren Ergebnisse gute Aussichten auf eine ökonomische Nutzung haben. Die Bereitschaft zur Förderung akademischer Grundlagenforschung hingegen nimmt stetig ab, da die Unternehmen die Input-Output-Relationen als zu ungünstig einschätzen. Industrielle Forschung wird auf diesem Weg zunehmend zu einer „Risikoreduzierenden Investition“ (Hack 1998): Einerseits sind die Unternehmen durch Eigenforschung unabhängiger vom externen technologischen Wandel, andererseits erhöht sich der Druck auf die Konkurrenz, ebenfalls in Forschung zu investieren (Hack 1998). Insgesamt ist dies die Phase, in der sich die wichtigsten Strukturmerkmale der „Industriewissenschaft“ (Krohn 1981) herausbilden, zu denen insbesondere der Bezug der Entdeckung auf eine konkrete Herstellungspraxis sowie die Überwindung disziplinärer Grenzen ${ }^{57}$ zählen.

„Die klassischen Theorien der Naturwissenschaften sind allesamt extreme Idealisierungen der Realität. (...) z.B. ist es unmöglich, aus irgendeiner Theorie einen Glühfaden herzuleiten, geschweige denn einen für die kommerzielle Fertigung ,idealen’. Dieses ,Ideal', das das Ziel einer wissenschaftlichen Lösung eines Herstellungsproblems ist, ist dem ,idealen Objekt' der klassischen Naturwissenschaft geradezu entgegengesetzt: Es wird nicht aufgespürt durch eine Strategie der systematischen Vereinfachung, sondern eine Strategie der optimalen Aggregierung.“ (Krohn 1981:13f. zitiert nach Hack 1985)

„Verlierer“ dieser Entwicklung sind die Universitäten. Zwar bleiben sie ein relevanter Wissenslieferant, doch haben sie im Vergleich zum Ende des 19. Jahrhunderts in den 1920er Jahren deutlich an Reputation in den Unternehmen verloren. Im Unterschied zu den chemischen Unternehmen, die unter wirtschaftlichen Aspekten kaum eine andere Wahl haben, als sich an die veränderten Rahmenbedingungen anzupassen und intern zu

\footnotetext{
${ }^{56}$ Exemplarisch für diese Effekte ist der enge Zusammenhang zwischen der Entfaltung der Farbstoffchemie und der Herstellung von Pharmazeutika: Beide basieren auf Steinkohlenteer als Rohstoff, darüber hinaus jedoch werden Farbstoffe bei der Arzneimittelforschung zum Einfärben von Gewebe genutzt. Und schließlich beruht auch eine medizinische Therapieform, die Chemotherapie, auf der Nutzbarmachung des Zusammenspiels von Farbstoffen und unterschiedlichen Krankheitserregern (Drews 1998).

${ }^{57}$ Diese Interdisziplinarität ist allerdings bezogen auf den gesamten Innovationsprozess zu verstehen. Zwischen den Bearbeitungskontexten der einzelnen Funktionsbereiche bildeten sich in der Industrieforschung schon früh strenge Übergabeprozeduren heraus, die nicht nur der Kontrolle des Bearbeitungsstands dienten, sondern auch die Verantwortungsbereiche voneinander abgrenzte (Hack 2001).
} 
kooperieren, behaupten sich in den Universitäten die parallel geordneten Disziplinen. Den Professoren bleibt angesichts sprunghaft steigender Studierendenzahlen, aufwendigerer Lehrverpflichtungen sowie eines immer eingeschränkteren Staatsetats kaum noch Zeit, um sich mit den Kollegen der anderen Fachdisziplinen auszutauschen.

Der Aufbau der industriellen Forschungskapazitäten ist für die Unternehmen ein teures Unterfangen, da neben den Ausgaben für Material und Ausstattung höhere Personalkosten sowie auch Investitionen in die Infrastruktur zu Buche schlagen. Insgesamt muss die Produktion zusätzlichen Wissens also in Verbindung gesetzt werden mit Investitionen in Human- und Sachkapital. Dass sich die Unternehmen dennoch in umfassender Weise für den langfristigen Aufbau eigener Forschungs- und Entwicklungskapazitäten entscheiden und ihr Kapital den Universitäten nur noch eingeschränkt zur Verfügung stellen, erklärt sich aus der Konkurrenzsituation auf dem Markt für Chemieprodukte. Mit der Ausdifferenzierung des Markts wird nicht der Wissensbestand eines Unternehmens, sondern sein Zugang zu zusätzlichem Wissen als Grundlage für innovative Produkte zum entscheidenden ökonomischen Faktor. Diese Form des Wissens ist aber nur dann für Unternehmen von Vorteil, wenn sie exklusiv und ausschließlich darüber verfügen können. Insofern ist die akademische Wissensproduktion zwar nach wie vor interessant für die Industrie, die Kontrolle über die expandierende akademische Forschung und die aus ihr hervorgehenden Ergebnisse wird indes immer komplizierter. Da die Reputation der Professoren zunehmend auf Veröffentlichungen basiert, verliert das in der Forschung geronnene Wissen seinen unter ökonomischen Aspekten relevanten exklusiven Charakter und wird als gesellschaftliches Wissen zum öffentlichen Gut (Stehr 2001). In der Chemischen Industrie war man sich schon früh darüber im klaren, dass auch naturwissenschaftliches Wissen nicht gänzlich explizierbar ist. Gerade für die Umwandlung von Innovationen in Endprodukte wird „tacit knowledge“ (Turner 1995) oder implizites Wissen (Polanyi 1985), also Wissen, das nur durch praktisches Tun und eigene Erfahrung erworben werden kann, benötigt. ${ }^{58}$ Dieses Wissen ist an Personen gebunden und kann nicht durch Bücher oder Theorie vermittelt werden. Im Gegensatz zum exakt beschreibbaren „knowing that“ liegt der Fokus der tacit knowledge auf dem „knowing how“ (Göranzon, Josefson 1988). In Anbetracht der Wissensexpansion sind Unternehmen mit der Herausforderung konfrontiert, externes Wissen erstens zu beurteilen und zweitens zu internalisieren. Die Beurteilungskompetenz ist aber ohne internes Know-how nicht zu gewährleisten: Nur wer selbst forscht, wer Spezialisten und zu integrativem Denken fähige Wissenschaftler hat, ist imstande, Nutzen und vor allem auch Risiko neuer Technologien und Substanzen, die „außen“ entwickelt werden, zu beurteilen. ${ }^{59}$ Und auch die Internalisierung von Wissen bedarf

\footnotetext{
${ }^{58}$ Da uns im Rahmen unserer Fragestellung vor allem die Perspektive der Akteure auf die Problemstellung wichtig ist, verzichten wir an dieser Stelle auf die Diskussion und Kritik der unterschiedlichen Ansätze zum Thema „Erfahrungswissen“ und ihrer epistemischen Grundlagen (vgl. weiterführend zum Umgang mit Erfahrungswissen in Unternehmen etwa Böhle 2002).

${ }^{59}$ Eine nicht uninteressante Analogie zu dieser Phase stellt die derzeitige Entwicklung im Bereich der Biotechnologie dar. Entgegen der Erwartung, dass sich die Wertschöpfungskette der Medikamententwicklung dahingehend ausdifferenziert, dass kleine, leistungsstarke Start-ups in der Hauptsache Forschungsaktivitäten betreiben und die etablierten Pharmaunternehmen die Entwicklung bis zur Marktreife voran treiben, können wir eine komplementäre Entwicklung ausmachen. Für erfolgreich am Markt agierende Big Pharma-Player gilt, dass sie in jedem Fall ihre Forschungsaktivitäten beibehalten bzw. um biotechnologisches Know-how erweitern. Denn im Rahmen von Kooperationen ist sowohl die Auswahl von Biotech-Unternehmen als auch eine effektive Zusammenarbeit sowie schließlich die Internalisierung von Wissen nur unter der Prämisse einer qualitativ hochwertigen Eigenforschung zu leisten (Drews 1998; Briken, Kurz 2002; Dolata 2002).
} 
immer eines Mindestmaßes an Eigenforschung, um die sozialen und kognitiven Rahmenbedingungen zur Realisierung und Anwendung von Wissen zu gewährleisten. Anders ausgedrückt: Die Unternehmen können erst durch den Aufbau eigener Forschungseinrichtungen Bestände expliziten wie impliziten Wissens aufbauen, die internes und externes Wissen verbinden und für die interne Forschung anschlussfähig zu machen.

\subsubsection{Wissenschaftler in der Produktion}

Der zunehmende Einsatz von Wissenschaftlern in den Chemieunternehmen ist insofern auch zu verstehen als Aufbau interner Innovationskompetenz. Bis ca. 1900 jedoch arbeiten Akademiker hauptsächlich innerhalb der Forschung und Entwicklung in den Laboratorien, die entweder hierarchisch gesehen oberhalb oder aber parallel zu den Produktionsbetrieben liegen. Zwar werden in Folge der Diversifizierung der Produktionsstätten von Chemikern geleitete Labors in den Betrieben zum neuen Standard. Die Kooperation zwischen Chemikern und der Produktion in Form eines Austauschs zwischen akademischem und Erfahrungswissen bleibt davon allerdings zunächst unberührt. Im Gegenteil, in den Unternehmen werden die Chemiker aus der Produktion fern gehalten, zu sehr misstraut man ihnen und befürchtet, dass sie Produktionsgeheimnisse veruntreuen. Dass diese Distanzierung auch von den Betriebsmeistern forciert wird, scheint sehr wahrscheinlich, betrachtet man das Statuskonzept der Betriebe. Bis auf wenige Ausnahmen wird die Leitung der einzelnen Produktionsstätten weiterhin vom „Meistersystem“ dominiert. Diese (Betriebs-) Meister haben in der Regel die „alten“ Berufe in den Färbereien gelernt (Färber, Dämpfer, Heizer, Küfner) und sind von den Fabrikgründern in die Produktion eingeführt. Mit der Expansion der Unternehmen wurde ihnen die Führung einzelner Produktlinien übertragen, ihre Kompetenzen gründen sich vor allem auf einem langjährigen Erfahrungswissen. So sind sie bereits in der Aufbauphase des Betriebs in die Planung eingebunden und übernehmen nach der Anlaufphase die Betriebsleitung in Verbindung und mit direkter Kontrolle über Produktion, Anlagen und Arbeitskräfte. Darüber hinaus wird ihnen eine weitreichende Autonomie bei der Gestaltung der Produktion eingeräumt. Ihr Mitspracherecht erstreckt sich von technischen Entscheidungen wie etwa der Einführung neuer Verfahren, bis zu Personalentscheidungen wie Anwerbungen, Kündigungen und Beförderungen innerhalb des Betriebes. ${ }^{60}$ In der Betriebsführung haben die Meister eindeutig das Sagen. ${ }^{61}$ Ihr betrieblicher, nicht kodifizierbarer Wissensbestand gilt den Unternehmen als wichtiges Humankapital, so dass ihre Bezahlung weit über dem der akademischen Funktionen liegt. Einem Wechsel der Meister in ein Konkurrenzunternehmen soll damit vorgebeugt werden. ${ }^{62}$

\footnotetext{
${ }^{60}$ Dies hieß bis ca. 1900, dass die Betriebsmeister sich ihre Arbeiter tatsächlich selbst, also auf der Straße, besorgen mussten, und in Zeiten knapper Arbeitskräfte mit anderen Meistern um die wenigen Arbeiter konkurrierten. „So begaben sich die Meister in den Pausen auf die Straße, in der Nähe des Pförtnerhäuschen und hielten Umschau, ob sie einen Arbeitslosen erspähten. Es kam hier manchmal unter den Meistern zu wilden Raufereien, wenn der eine oder andere einen dingfest machte. Bei der großen Nachfrage wurde nicht auf Qualität geachtet. Auswahl gab es nicht.“ (Erfahrungsbericht W. Peper, Elberfeld, um 1890, zitiert nach Peetz (1981: 153).

${ }^{61}$ So schreibt der Chemiker Arnold Fischer um 1880 über seine Arbeit in der Alizarinfabrik bei Bayer in Elberfeld: „Meine Tätigkeit hing vollständig ab von der Laune des Meisters der Alizarinfabrik, da dieser der Leiter derselben (...) Gefiel es dem Meister, mich länger bei der Arbeit zu halten, so schickte er mir abends gegen 7 Uhr noch Fabrikanalysen mit der Bemerkung, die Kessel sollen heute Abend noch beschickt werden, ich habe die Analysen nötig’.“ (Peetz 1981:51f.).

${ }^{62}$ Ein Zitat aus dem Jahr 1880 belegt dies und zeigt, dass die Monopolisierung des Wissens bei den Meistern auch als Maßnahme gegen die Konkurrenz genutzt wurde: „Um die Konkurrenz sich vom Halse zu halten, arbei-
} 
Erst seit Beginn des 20. Jahrhunderts setzen die Unternehmen systematisch Chemiker als Betriebsleiter ein. Um Statuskonflikte zu vermeiden, behalten die Meister in den alten Betrieben zumindest formal ihre Position, die Kontrolle der betrieblichen Prozesse hat aber der Chemiker des Betriebslabors. ${ }^{63}$ In den neu errichteten Produktionsanlagen, die nach neuesten Verfahren innovative Produkte herstellten, wird schließlich das Meisterprinzip aufgegeben. Dort setzen die Unternehmen akademisch ausgebildete Wissenschaftler bzw. Chemiker als Betriebsleiter für Managementaufgaben wie auch zur Leitung der Eigenforschung im Betriebslabor ein. Für den Produktionsbereich bedeutet dies eine eindeutige Zäsur, der den Erfahrungsträgern aus der Produktion einen deutliche geringeren Stellenwert innerhalb des Innovationsprozesses zuweist als zuvor.

\subsubsection{Zwischenresümee}

Innerhalb von vierzig Jahren wandelt sich die Chemische Industrie von einer auf Erfahrungswissen(schaft) basierenden Branche durch die Internalisierung chemischakademischen Wissens zu einer science based industry. Seitdem basiert die Chemische Industrie auf dem Modell der integrierten Industrieforschung, deren Hauptanteil zwar anwendungs- und problembezogene Forschung ist, in dem aber auch die Grundlagenforschung eine wichtige Rolle spielt. Das unternehmerische Kalkül ist es, nicht nur den Forschungsbedarf der einzelnen Fachbereiche abzudecken und inkrementelle Verbesserungen voranzutreiben, sondern auch Basisinnovationen zählen zum Kompetenzbereich der unternehmensinternen Forschung. Obwohl sich die Unternehmen davon nicht immer einen direkten Nutzen versprechen können, gelten Investitionen in diesem Bereich als strategische Optionen für Zukunftsmärkte und Produkte. Der Vorteil der Internalisierung liegt in der Möglichkeit zur internen Kontrolle der Ergebnisse: Während extern generiertes Wissen auch durch die Konkurrenz nutzbar wäre, schließt eine internalisierte Forschung dies aus und verhindert so einen kräftezehrenden Preiskampf um die Nutzungsrechte. Darüber hinaus erhöhen umfassend ausgestattete Forschungsabteilungen die Potentiale zur Verwertung externer Resultate. Deutliches Zeichen dafür ist die Akademisierung der Produktion: Chemiker bzw. das mit ihrer Ausbildung verbundene Wissen um chemische Prozesse und ihre Methodenkompetenz diffundieren in alle Unternehmensbereiche. Im Unterschied etwa zu Unternehmen in den Fertigungsindustrien finden sich Forschungs- und Entwicklungsaktivitäten auch in den einzelnen Produktionsstätten bzw. in den Betriebslaboratorien. Das betriebliche Management betreibt hier bis heute einen Teil Eigenforschung. In diesem Prozess begründet sich die Rolle Deutschlands als first mover in der Kapitalisierung von Wissen. Die systematische Organisation angewandter Forschung in den Industrieunternehmen forciert die industriellen Innovationen insbesondere in der Chemie (Chandler 1990; Weingart 2001). Im folgenden wird zu zeigen sein, wie wissen-

teten wir mit falschen Thermometern, längeren Thermometern, ungefähr einen Meter lang. Jedes Thermometer zeigte hundert Grad zuviel, denn wir wurden hart von der Konkurrenz bedrängt: Die besten Arbeiter wurden uns stets von ihr entzogen. Sie zahlten für die Woche 30 Thaler, und dadurch eben, dass die Leute nichts anderes wussten, sagten sie die falsche Temperatur.“ (Peetz 1981: 24).

${ }^{63} \mathrm{Zu}$ vermeiden sind Konflikte letztlich nicht. So setzen sich in einigen Fällen Meister gegen die „Bedrohung“ ihrer Hausmacht zur Wehr, in dem sie den Chemikern den Zutritt zur Produktion verwehren und sie dazu anhalten, sich auf die Analytik im Labor zu beschränken. 
schaftliche und technische Kompetenzen zum Gegenstand formaler Ausbildungsprozesse werden, und immer ausdifferenziertere arbeitsteilige Strukturen entstehen. ${ }^{64}$

\subsection{Ausdifferenzierung der Berufe in der Chemischen Industrie}

Seit den 1880er Jahren verstärkt sich, nicht zuletzt bedingt durch das rasante Wachstum der Branche, auch in der Chemischen Industrie die horizontale und vertikale Arbeitsteilung. Die Verfahrenstechnik und die Instandhaltung der Anlagen werden zu wichtigen Variablen im unternehmerischen Rationalisierungskalkül, da sich mit der Vergrößerung der Anlagen das Anlagendesign nicht mehr in direkter Analogie zu den Laborinstrumenten gestalten lässt Wo zunächst noch Chemiker unterstützt durch Handwerker in einem empirisch geprägten trial and error -Verfahren Produktionssysteme erproben, reicht dies beim Überschreiten kritischer Größenordnungen nicht aus. Technische Probleme der Produktionsanlagen sind nicht mehr durch eine partielle Verbesserung bekannter Verfahren zu lösen. Ein neuartiges physikalisch-technisches Wissen wird benötigt, neue Anforderungsprofile entstehen und neue Kompetenzen werden zur Organisation und Umsetzung von Innovationen eingesetzt. Neben Chemikern, Unternehmern und un- und angelernten Arbeitskräften differenziert sich die Personalstruktur in akademisch ausgebildete Berufsgruppen wie Physiker, Architekten und Verfahrensingenieure ${ }^{65}$ sowie verschiedene Handwerkergruppen.

\subsubsection{Organisatorische und personelle Ausdifferenzierung im akademischen Bereich}

Bis etwa in die 1880er Jahre hinein sind fast ausschließlich Chemiker im Bereich der Forschung und Entwicklung für die Umsetzung von Innovationen, d.h. für die Planung und Konstruktion der Produktionsverfahren zuständig. Sowohl die Dynamik des Marktes, Zeit- und Kostendruck wie auch die immer aufwendigeren Verfahren verlangen jedoch eine organisatorische und personelle Neustrukturierung der Organisation zur technischen Umsetzung der Innovationen. Die Zusammenarbeit von Experten verschiedener Fachrichtungen löst die individuellen Umsetzungsstrategien bei der Entwicklung der für die Massenproduktion notwendigen Betriebsmittel wie Gebäude, Apparate, Produktionstechnikund Anlagen ab. Vor allem Ingenieure treten bei der Konstruktion der Produktionsanlagen auf den Plan und verfolgen die verfahrenstechnische Umsetzung. Sie sind aufgrund ihrer Spezialisierung in der Lage, die neuen Produktionsstätten wesentlich kurzfristiger, dauerhafter, kostengünstiger und leistungsfähiger zu planen und zu bauen, als die „technischen Chemiker“ (Christ 1995:16).

Eine wesentliche Grundlage bildet die Etablierung der Technischen Hochschulen in Deutschland, die zwischen 1860 und 1880 eine entwickelte Infrastruktur an technischen Ausbildungsstätten darstellen, deren Ausbildungsinhalte eng an den Bedürfnissen der

\footnotetext{
${ }^{64}$ Eine ähnliche Entwicklung wie die Chemische Industrie nimmt lediglich die elektrotechnische Industrie, vgl. dazu König (1995), Weingart (2001).

${ }^{65}$ Diese Tendenz unterstreicht der Wandel in der Zusammensetzung des 1850 gegründeten VDI. „The position of engineers in the workplace changed considerably after 1871 . Whereas many members of the VDI were engineerentrepreneurs in that year, by 1900 most German engineers were employees in increasingy large enterprises [...]" (McClelland 1980:91).
} 
Industrie orientiert sind. Konnte die Chemische Industrie bereits in hohem Maß von den Absolventen der staatlichen Hochschulen profitieren, stehen ihr nun auch Absolventen zur Verfügung, deren Ausbildung auf den Bedarf der noch jungen Industrie direkt zugeschnitten ist. Verstärkt werden die Synergieeffekte in der Folgezeit durch den Wandel des Selbstverständnisses der Technischen Hochschulen. Die Ingenieure streben sowohl im Hinblick auf den akademischen Titel als auch auf die soziale Anerkennung mit den Absolventen der Universitäten eine Gleichstellung an. Dies führte zu einer Übernahme der universitären Standards in Lehre und, relevanter noch, in der Forschung. Organisatorisches Instrument sind Laboratorien, die nun auch an den THs zur Verbindung von Theorie und Praxis beitragen sollen. Die technischen Erfahrungen aus den Industrieunternehmen können fortan mit den theoretischen Kenntnissen über naturwissenschaftliche Zusammenhänge zu einer spezifischen Methodologie der Ingenieurwissenschaften verkoppelt werden. Diese Akademisierung der Ingenieurausbildung wird vom Management der Industrieunternehmen zunächst durchaus skeptisch beurteilt, man befürchtet eine zu große Distanz der Ingenieure von der industriellen Praxis. Doch ganz im Gegenteil konnte die Industrie nun die Integration wissenschaftlichen Wissens und akademischer Kompetenz in noch breiterem Umfang nutzen.

„Waren die Professoren an den THs bis zur Jahrhundertwende vor allem Lehrer, deren Beziehungen zur Industrie in Beraterfunktionen oder auch in der Leitung von Forschung innerhalb der Firmen bestand, so intensivierten sich diese Verbindungen aufgrund der hochschuleigenen Forschungstätigkeiten zu Kooperationsbeziehungen.“ (Weingart 2001:180)

Ohne diese Verwissenschaftlichung der Ingenieurwissenschaften und den Wissenstransfer zwischen akademischer und industrieller Forschung wäre die verfahrenstechnische Umsetzung einer chemischen Reaktion vom Reagenzglas ins Laboratorium in die Metallapparatur des Betriebes oft genug nicht möglich gewesen (Wetzel 1991).

Der Boom der Ingenieurwissenschaften an den THs findet seine spiegelbildliche Entsprechung in den Industrieunternehmen. Verstärkt differenziert sich die Arbeitsteilung jenseits der Linie Chemiker / Unternehmer auf der einen und Arbeiter auf der anderen Seite aus. Das Gros der Chemiker konzentriert sich in der Forschung. Bindeglied zwischen Zentralforschung und Produktion sind die Chemiker in der Betriebsleitung. In den prosperierenden Unternehmen steigt parallel dazu der Anteil an Ingenieuren, die verfahrenstechnische Forschung und Entwicklung zur Umsetzung und Durchführung der Produktionsprozesse betreiben. Markante Beispiele hierfür sind Hochdruckverfahren wie die Ammoniaksynthese, die Methanolsynthese und die Kohlehydrierung, die von Anfang an Vorstellungen und Denkweisen des Chemikers und des Physikers mit denen des Ingenieurs verbinden. Dies entspricht dem Selbstbild der Ingenieure, die ihre wissenschaftliche Ausbildung als „Angehöriger desjenigen Berufsstandes, der aufgrund seiner Ausbildung nicht nur imstande, sondern geradezu berufen war, Wissenschaft in praktisch nutzbare Technik umzusetzen“" verstehen (Burchardt 1992:12). ${ }^{66}$

\footnotetext{
${ }^{66}$ Ein Beispiel für das Selbstverständnis und bewusstsein zeigt sich im „Ingenieurslied“ von Heinrich Seidel (1903): „Die Ingenieure sollen leben! In ihnen kreist der wahre Geist der allerneusten Zeit! Dem Fortschritt ist ihr Herz ergeben, dem Frieden ist hienieden ihre Kraft und Zeit geweiht! Der Arbeit Segen fort und fort, Ihn breitet aus von Ort zu Ort, von Land zu Land, von Meer zu Meer - Der Ingenieur!“ (zitiert nach Christ 1995).
} 
Die Verfahrenstechnik ${ }^{67}$ insgesamt trägt zur Lösung von Problemen bei, die aus der skalenökonomischen Rationalisierung sowie der chemisch-technischen Entwicklung resultieren. ${ }^{68}$ Diese Ausdifferenzierung der ingenieurwissenschaftlichen Disziplinen innerhalb der Chemischen Industrie wird auf zwei Ebenen wirksam. Erstens stehen die Chemieunternehmen in einer unumkehrbaren Abhängigkeit von der Verfahrenstechnik, die sich als zweites wissenschaftliches Standbein etabliert. Zweitens entstehen organisatorisch unterschiedliche Disziplinen nebeneinander, von deren Kooperation und Koordination der wesentliche Erfolg der Unternehmen abhängt und die den reibungslosen Ablauf der Produktionsprozesse maßgeblich beeinflussen, die Chemiker verlieren ihre wissenschaftliche Hegemonie. ${ }^{69}$ Im Rahmen des chemisch-verfahrenstechnischen Geschehens wird die Zusammenarbeit von Chemikern und Verfahrensingenieuren nicht nur in der Planung und der Entwicklung, sondern auch in der alltäglichen Betreuung der Anlagen wichtig. Organisatorisch tragen die Unternehmen diesem neuen Sachzwang Rechnung, indem die Verfahrenstechniker eine dem Betriebsleiter zugeordnete Funktion einnehmen. Der Ingenieur ist dann sowohl für die anlagentechnischen Probleme zuständig, als auch stellvertretender Betriebsleiter. Die Verfahrenstechniker bleiben hierarchisch zentralen technischen Abteilungen (in der Regel das sogenannte Ingenieurwesen) unterstellt, während die Betriebsleiter ebenso wie die anderen im Produktionsbetrieb Beschäftigten den einzelnen am nach Endprodukten definierten Geschäftsbereichen zugeordnet sind (Tacke 1997).

\footnotetext{
${ }^{67}$ Die Einsatzfelder der Verfahrensingenieure sind vielfältig und zeigen, wie stark sich die chemische Produktion ausdifferenziert hat. In Forschung und Entwicklung arbeiten Verfahrensingenieure an experimentellen und theoretischen Untersuchungen zur mathematisch-physikalisch-chemischen Beschreibung der Grundverfahren und der technisch-wirtschaftlichen Gestaltung der Maschinen und Apparate zur Durchführung der Grundverfahren sowie an der Entwicklung von Verfahren auf der Basis des Systemzusammenwirkens und der systematischen Entwicklung von Anlagen von Produktionsprozessen. In der Planung, Konstruktion sowie dem Apparate- und Anlagenbau planen und errichten Verfahrensingenieure technische Anlagen, bestehend aus Apparaten, Maschinen, Rohrleitungen, Mess- und Regelgeräten etc., die umweltfreundlich und von hoher Betriebssicherheit sein müssen. Verfahrensingenieure in Betrieb und Produktion sorgen für einen reibungslosen Ablauf der Prozesse in den Produktionsanlagen sowie die Instandhaltung und Pflege des Produktions-Know-hows. Sie organisieren, leiten oder überwachen die Inspektion, Wartung und Montage der Anlagen. Darüber hinaus geben sie Anstöße zur Verfahrensverbesserung, die im Rahmen der Betriebstechnik umgesetzt werden. Verfahrensingenieure in Anwendungstechnik, Ein- und Verkauf analysieren und prognostizieren Markt- und Absatzchancen der Produkte, verhandeln mit Zulieferern und Kunden oder beraten Käufer verfahrenstechnischer Anlagen. In den Bereichen Verwaltung, Technischer Überwachung und dem Patentwesen arbeiten die Ingenieure schließlich als Experten und Berater mit. Vgl. „Verfahrenstechnik / Chemieingenieurwesen“ GVC - VDI-Gesellschaft 1997.

${ }^{68}$ Auch auf Verbandsebene nimmt man sich um 1920 dieser Problematik an: „Wie oft scheiterte schon manches schöne und geistvoll erdachte, im Labor sicher arbeitende Verfahren im Betriebe an der Apparaturenfrage." Dieser Gedanke, veröffentlicht in einer Denkschrift im Jahr 1920, hatte den Wissenschaftler und Unternehmer Max Buchner zwei Jahre zuvor dazu bewegt, aus dem Verein Deutscher Chemiker eine Fachgruppe für chemisches Apparatewesen herauszulösen. Hintergrund war ein tief greifender Wandel in der damaligen Chemieindustrie: Die moderneren Verfahren benötigten immer kompliziertere Apparaturen. Dem Chemiker aber fehlte zu deren Konzeption häufig die technische Kompetenz, und er versicherte sich nicht "zur rechten Zeit des Rates und der Mitarbeit tüchtiger Ingenieure", wie Buchner feststellte. Er forderte deshalb eine engere Zusammenarbeit von Chemikern und Technikern und rief zu diesem Zweck die separate Fachgruppe ins Leben. Aus dieser entstand im Jahr 1926 die Dechema, Deutsche Gesellschaft für Chemisches Apparatewesen e.V. (Lindner 2001).

${ }^{69}$ Dieser Prozess ist nicht friktionsfrei abgelaufen, denn die an den Universitäten ausgebildeten Chemiker und Physiker sahen zunächst in den Absolventen der Technischen Hochschulen „Emporkömmlinge“, deren wissenschaftliche Reputation sie anzweifelten. Die Ingenieure mussten für eine Anerkennung ihrer Polytechniken und Bildungsabschlüsse als gleichwertig im Vergleich zu den traditionell akademischen Berufen kämpfen und betonten, dass sie während der Ausbildung eine qualitativ vergleichbare wissenschaftliche Grundlage erhalten, die sich lediglich durch ihren engeren Bezug zur industriellen Praxis von der naturwissenschaftlichen Hochschulausbildung unterscheidet (Weingart 2001).
} 


\subsubsection{Diversifizierung und Spezialisierung in der Instandhaltung}

Die Instandhaltung basiert von Beginn an auf handwerklichen Kompetenzen. Schmiede, Schlosser sowie Zimmermänner zählen zu den handwerklichen Berufsgruppen, die als erste „Instandhalter“ für die Behebung von Störungen im Ablauf der einfachen Produktionsvorgänge zuständig sind und meist in kleinen Werkstätten in die ersten chemischen Betriebe integriert waren. Ihre Arbeit beschränkt sich auf Reparaturen von wenig komplexen Anlagen und Hilfsmitteln, d.h. die qualifikatorischen Anforderungen entsprechen dem in der Ausbildung erlernten. Erst mit der fortschreitenden Technisierung der Verfahren erfährt die Instandhaltung der Anlagen entscheidende Veränderungen. Die Komplexität der Anlagen macht diese störungsanfälliger und ein Ausfall der Anlage bedeutet immer auch kostspielige Produktionsverzögerungen oder Produktionsstillstände für die Unternehmen. Fehlendes oder unqualifiziertes Personal aus den Reparaturbetrieben verzögert immer häufiger die Reparaturen mit der Folge, dass die Instandhaltung zu einer wichtigen strategischen Größe bei den Effizienz- und Rationalisierungsüberlegungen der Unternehmer wird. Rasch entstehen spezialisierte Reparaturbetriebe und „mechanische Werkstätten“, die mit einem hohen Sach- und Personalaufwand ausgestattet werden. ${ }^{70}$ Doch nicht nur der zunehmende Bedarf an qualifizierten Handwerkern führte zu dieser engeren Anbindung der Instandhaltung an die einzelnen Betriebe. Die Sorge vor einem Einblick der Konkurrenz in die eigene Arbeitsweise ist gerade in der Gründerphase der Branche hoch, und so wird der Apparatebau nur ungern an externe Werkstätten vergeben. Jeder Abteilung wird daher ein leitender Ingenieur zugewiesen, der sowohl die Reparaturaufträge des Alltagsgeschäfts überwacht, als auch den Bau neuer Anlagen vorbereitet und begleitet (Christ 1995:22).

Daneben verändern sich mit der Technisierung die qualifikatorischen Anforderungsprofile der Handwerker. Während die Reparaturarbeiten zunächst noch ganz den Qualifikationsanforderungen des metallverarbeitenden Handwerks entsprechen, werden in immer stärkeren Maß prozessspezifisches chemisch-technisches Verständnis sowie analytische Denkfähigkeiten nötig, um die Anlagen fachgerecht instand zu halten. Die allgemeinen Fachkenntnisse aus den Bereichen Maschinenbau oder dem Schlosserhandwerk reichen für die heterogenen und aus dem spezifischen Aufbau eines Chemiebetriebs resultierenden Anforderungen nicht mehr aus. Je weiter die naturwissenschaftliche und betriebswirtschaftliche Optimierung voranschreitet, desto deutlicher wird die Diskrepanz zwischen den in Volksschule und Handwerk vermittelten Qualifikationen gegenüber den betrieblichen Anforderungsprofilen. Konsequenzen für die betriebliche Aus- und Weiterbildung hat dies anfangs nicht, denn obwohl die Anzahl der Beschäftigten in den Reparaturbetrieben zwischen 1865 und 1914 überproportional zum Wachstum der Gesamtarbeiterzahl ansteigt, ${ }^{71}$ bleibt die interne berufliche Erstausbildung von Lehrlingen auf Einzelfälle beschränkt. Erst in den 1910er Jahren bauen die Chemieunternehmen Lehrwerkstätten auf, in denen systematisch und quantitativ relevant Facharbeiter für die Instandhaltung ausgebildet werden. Diese Entwicklung ist allerdings keine chemiespezifische, sondern ver-

\footnotetext{
${ }^{70}$ Der hohe strategische Stellenwert lässt sich daran ablesen, dass die Werkstätten bereits sehr früh eingerichtet werden und eine im Verhältnis zur Gesamtbelegschaft hohen Beschäftigtenanteil aufweisen. In den Farbwerken Hoechst sind bereits 1871 von 250 Arbeitern 40 in den Mechanischen Werkstätten tätig ( Christ 1995:22).

${ }^{71}$ Da sich in den Firmenarchiven keine Aufschlüsselung der Gesamtarbeiterzahlen nach Sparten und Abteilungen findet, schließt Christ (1995) dies aus der flächenmäßigen Ausdehnung einzelner Abteilungen im Untersuchungszeitraum.
} 
weist auf den generellen Trend der Wirtschaft, die Ausbildung des „Nachwuchses“ selbst in die Hand zu nehmen, und so setzten sich „Ausbildungsberufe“ („Lehr- und Anlernberufe“) Anfang des 20. Jahrhunderts in der gesamten Industrie durch (Hesse 1968: 109).

Das skizzierte Muster von Arbeit gerät im Rahmen automatisierter Produktionsprozesse, wegen funktionaler Notwendigkeiten und im Hinblick auf die Defizite der vorherrschenden Arbeitseinsatzpolitik unter einen wachsenden Anpassungsdruck. Am Gewicht der empirisch-praktischen Fähigkeiten verändert sich prinzipiell nur wenig, aber Theorieaufwand und Schulungsbedarf steigen rapide an. In den neuen Produktionssystemen steht nicht mehr die einzelne Maschine im Zentrum der Aufmerksamkeit, sondern der reibungslose, effiziente Ablauf des gesamten Produktionsprozesses, und parallel dazu differenzieren sich die Tätigkeiten in der Instandhaltung in mechanische sowie elektrotechnische. Als Konsequenz aus dem wachsenden Anteil pneumatischer Regler wird 1960 der Mess- und Regelmechaniker zum anerkannten Lehrberuf. Die hochwertigen und komplexen Prozessleiteinrichtungen, die mit der fortschreitenden Modernisierung der Produktion seit den 1980er Jahren Einzug in die chemische Industrie halten, lassen aber auch diesen neuen Beruf schnell veralten und im Rahmen der Neuordnung der Elektroberufe ersetzt 1992 der Ausbildungsberuf des Prozessleitelektronikers den Mess- und Regelmechaniker.

Das traditionelle Arbeitskraftmuster der Instandhaltung basiert auf einer hohen Autonomie, die sich vor allem auf den von Anfang der Industrialisierung an bestehenden Facharbeiterstatus stützt. Auch die Verakkordierung von Reparaturen, wie sie sich eine Zeit lang im Rahmen der Expansion und Ausdifferenzierung der Chemieunternehmen in Teilen der Werkstätten durchsetzte, hat an dieser Situation nichts grundsätzliches ändern können. Die Instandhalter besetzen eine wichtige Rolle bei der Aufrechterhaltung und Gewährleistung des Produktionsprozesses. Diese sichert ihnen ein hohes Anerkennungspotential, das sich insbesondere beim Beheben von Störungen, also in der „Feuerwehrfunktion“ immer wieder neu formiert. Hinzu kommt, dass der einzelne Handwerker nach wie vor weitgehend selbständig im Meisterbetrieb oder in der Werkstatt arbeitet. Der Arbeitsalltag ist gekennzeichnet durch Formen prozessnaher Einarbeitung auf Basis von Fachwissen sowie der individuellen Spezialisierung von Arbeitern für bestimmte Betriebsteile. Diese Form der Spezialisierung führt aber nicht zu einer Dequalifizierung durch fehlende fachliche Herausforderungen. Im Gegenteil, da die Handwerker nach wie vor eine enge Anbindung, und zwar fachlich wie sozial an die Zentralwerkstatt haben, wird ihr allgemeinfachliches Kompetenzprofil noch um betriebsspezifische Spezialfertigkeiten erweitert.

Die Organisation der instandhalterischen Arbeiten ist für die Chemische Industrie bis heute von beträchtlicher wirtschaftlicher Brisanz. Die Instandhaltung prägt die Kosten- und Arbeitsstrukturen der Chemischen Industrie wie in keiner anderen Branche, und nach Schätzungen des „Trendreport Rationalisierung“ sind etwa 60 Prozent der operativen Tätigkeiten instandhalterischer Art, jeweils zu gleichen Teilen in den Fachabteilungen Metall und Elektro (Schumann et al. 1994). Der planbare Anteil (Jahresstillstände ganzer Anlagen, Routinewartungen oder Gebäudesanierungen) wird wegen oftmals beträchtlicher Lohndifferenzen, wo dies technisch möglich ist, an Fremdfirmen vergeben. ${ }^{72}$ Die

\footnotetext{
${ }^{72}$ Diese Differenz resultiert aus der Tatsache, dass die in den Chemieunternehmen arbeitenden Handwerker nach dem internen Entgelt bezahlt werden, d.h. sie profitieren auch von den übertariflichen Leistungen. Fremdfirmen sind meist wegen dieser Lohndifferenz günstiger.
} 
hohen Sicherheitsauflagen bedingen aber selbst bei diesen planbaren Tätigkeiten der Prozesswartung und Sicherung, dass Sicherheitseinweisungen für die externen Handwerker stattfinden und in den meisten Fällen eine Überwachung der Arbeiten durch die interne Instandhaltung. Für die permanent auftretenden Störungen in den Betrieben sind die unternehmenseigenen Fachkräfte zuständig. Harte Sicherheitsauflagen verhindern auch hier, dass diese Arbeiten vom Produktionspersonal übernommen werden können bzw. dürfen.

Bedingt durch die Automatisierungs- und Digitalisierungsbestrebungen, die sich seit den 1970er Jahren abzeichnen, steigt auf dem Shop floor der Bedarf an einer Gewerke übergreifenden Zusammenarbeit. Für die Arbeit in der Instandhaltung folgt daraus eine (durch sachlich nicht eindeutig zuzuordnende Qualifikationsanforderungen wachsende) horizontale Integration der verschiedenen Fakultäten (Chemie, Elektro, Metall). Insbesondere das Zusammenwirken von Anlagen- und Verfahrenstechnik einerseits und stofflich und organisatorischen Bedingungen der Produktion andererseits wird zum Gegenstand der Instandhaltung. Die Rückkopplung der Arbeit in der Instandhaltung an das Erfahrungswissen der Beschäftigten in der Produktion gewinnt an Bedeutung für eine kompetente Reparatur, beispielsweise wenn es zur Einschätzung und Fehleranalyse einer Störung unerlässlich ist, zu erfahren, wann und wie oft bestimmte Unregelmäßigkeiten auftreten. Alles in allem steht sowohl das Modell der Arbeitsteilung zwischen Produktion und Technik als auch das Modell der Arbeitsteilung innerhalb der Technik in Form einer durchgängigen Spezialisierung auf die Gewerke zunehmend im Widerspruch zum Prozessgedanken der Produktion. Damit konturiert sich Ende der 1980er Jahre eine Problematik für die Arbeitsvollzüge auf dem Shop floor, der die Chemische Industrie eher tentativ begegnet. Reorganisationsmaßnahmen bleiben auf die betriebsorganisatorische Ebene beschränkt.

\subsection{Produktionsarbeit in der Chemischen Industrie}

Wissenschaftsbasierung, ökonomischer Erfolg, Ausdifferenzierung der Produktpalette, wachsende Komplexität der Verfahren sowie zunehmende Spezialisierung im Bereich der Forschung und Entwicklung bilden die spezifischen Charakteristika der Chemischen Industrie. Waren Innovations- und Produktionsprozess in der Take-off Phase der Chemischen Industrie noch eng miteinander verbunden, wurde mit der Ablösung der Meisterwirtschaft auch die Ablösung der Innovationsfunktionen von nicht-akademischen Arbeitskräften eingeleitet. Die Organisation von Innovation ist zunehmend verwissenschaftlicht und durch die Etablierung von Betriebslaboratorien sowie die Einbindung der nun akademisch ausgebildeten Betriebsleitung bis in die Produktion ausgeweitet. Die unternehmensinterne Verkopplung von Forschung, Entwicklung und Produktion basiert vor allem auf akademisch-naturwissenschaftlichen Kompetenzen.

Im folgenden wird die Anforderungsseite der Arbeit in der direkten Produktion und deren Wandel im Rationalisierungsverlauf dargestellt, um anschließend die Entwicklung des Kompetenzprofils der Beschäftigten im Rahmen dieses Wandels der Chemischen Industrie zu analysieren. 


\section{Anforderungsprofile und technischer Wandel}

Die Arbeit in den ersten Chemiebetrieben ist, wir haben dies bereits gezeigt, eine Art „industrieller Kochkunst“ (Landes 1999) und dementsprechend sind die von den Beschäftigten zu leistenden Tätigkeiten vor allem körperlicher Art: Töpfe und Kessel füllen, erhitzen und die Temperatur beobachten. Beim Erreichen der vorgegebenen Temperaturen werden weitere Stoffe hinzugefügt, wieder muss per Hand gerührt werden und je nach Rezeptur wiederholt sich das Procedere. Am Ende eines Produktionsprozesses oder Prozessschritts werden die Behälter von den Arbeitern entleert und gereinigt. Erst wenn die Geräte abgekühlt sind, wird eine neue Charge angesetzt. ${ }^{73}$ Die Hauptaufgabe der Arbeiter ist es, die Produktionsbedingungen zur Durchführung einer Vielzahl chemischer Reaktionen zu gewährleisten. Chemiearbeit in ihren Anfängen, d.h. um 1860-1870, zeigt damit in der Qualifikations- wie der Regulationsdimension ein geringes Anforderungsniveau und kann als einfache Handarbeit bezeichnet werden, die kaum Schnelligkeit und Geschick verlangt oder ein spezifisches Wissen um die chemischen Prozesse voraussetzt. Die Kontrolle sowie die Angaben zu den Rezepturen liegen nicht im Aufgabenbereich der Arbeiter, sondern werden vom Betriebsleiter (Meister) erledigt. Da die Arbeit nicht besonders attraktiv ist, sind die angeworbenen Arbeitskräfte meist Wanderarbeiter, die für einige Zeit in den neuen Fabriken arbeiten und nach einigen Wochen weiterziehen. Die Fluktuation ist entsprechend hoch, Hauptgrund sind die hohen körperlichen Anforderungen sowie nicht zu unterschätzende gesundheitliche Gefährdungen: Giftige Dämpfe steigen ungehindert auf, Hitze und Gestank gehören zum Arbeitsalltag (Verg 1988).

Die hohen Fluktuationsraten sind zwar im Hinblick auf die kontinuierliche Aufrechterhaltung des Arbeitsprozesses kein unlösbares Problem, da die Arbeit aus wenigen, schnell zu lernenden Handgriffen besteht. Das Erfahrungswissen der Arbeiter ist minimal und kann kurzfristig durch die Einstellung einer neuen Kraft substituiert werden. Problematisch für die Unternehmer ist allerdings, die Motivation der Beschäftigten im Arbeitsprozess aufrecht zu erhalten. Im Gegensatz zu den Fertigungsindustrien ist es unter Berücksichtigung der kontinuierlichen chemischen Stoffumwandlungsprozesse nicht möglich, als Anreiz einen Stücklohn einzuführen.

„Die Steigerung der Leistung ist zumeist [...] auf geeignete Transportvorgänge und auf die Abstellung von Betriebsstörungen beschränkt. [...] Andererseits bedeuten Störungen des Roh- und Hilfsstoffzuflusses oder infolge Schäden der Apparatur für die chemischen Betriebe außerordentliche Belastungen, so dass die Werke eine Vermeidung solcher Störungen oder ihre entsprechende Beseitigung durch entsprechende Gestaltung der Löhne zu beeinflussen sucht.“(Mittler 1930:55)

\footnotetext{
73 „Alles beruhte auf Handarbeit.[...] Ich mußte nämlich Pötte ansetzen, die hatten auf einer Seite ein Loch, so daß man mit einer eisernen Stange rühren konnte. Hinein kamen 24 Pfund Fuchsin und 48 Eiweiß. Es war auch ein Thermometer da; wenn das bis 150 Grad stieg, dann wurde der Deckel mit Blut und Kalk beschmiert, daß er dicht wurde, damit er noch heißer werden konnte. Wenn der Topf nun gut war, wurde die Masse durch Filzhüte filtriert, die Farbe blieb im Hut sitzen.“ (Verg 1988: 32)
} 
Um dennoch mehr Arbeitsanreize schaffen zu können, führen die Unternehmen besondere Zulagensysteme ein, die für die hohen körperlichen Belastungen entschädigen sollen, und versuchen, die wechselseitige Kontrolle und kollektives Verhalten durch Prämienlöhne zu verstärken. $^{74}$

Mit der Ausweitung der Produktmengen setzt um 1880 die Mechanisierung der chemischen Produktion ein. Statt mit der Hand und dem Rührstab wird mit mechanischen Rührwerken gearbeitet, die Kessel werden mit Deckeln verdichtet und mit Abläufen versehen. Während sich zu Beginn des 20. Jahrhunderts mit der Entwicklung der Fließbandarbeit im Bereich der Endprozesse (Verpackung, Abfüllung von Endprodukten) in der chemischen Industrie Anzeichen für eine Mechanisierung mehren, bleiben die Kernprozesse der Stoffumwandlung nach wie vor auf eine diskontinuierliche Produktionsweise beschränkt. Die Arbeitskräfte übernehmen Befüllung, Bewachung, Wartung und Entleerung der Kessel, Bottiche und Tiegel (Bönig 1993). Die einzelnen Aggregate erfordern eine Einzelbedienung, Überwachungs- und Transportleistung. Der technische Wandel bleibt auf die Gestaltung der Apparatur beschränkt und führt nicht zu einer grundlegenden Veränderung des Anforderungsprofils der Chemiearbeiter. Qualitative Ansprüche an die Produkte verlangen zwar eine „sorgfältige und aufmerksame Arbeit“ (Christ 1995: 33 ), die Arbeitsbedingungen stehen allerdings in einem eklatanten Widerspruch zu diesen Anforderungen, die Produktionstätigkeiten werden als „eine schmutzige, unhygienische Arbeit an den zu engen Produktionsstätten..., welche ein Anbringen von Sicherheitsvorkehrungen nicht erlaubte“ (ebd.) beschrieben.

Zu einer qualitativen Veränderung der Chemiearbeit kommt es erst im Rahmen der nach dem Ersten Weltkrieg einsetzenden und seit den 1950er Jahren intensiv vorangetriebenen Automatisierung der Anlagen. Ursprünglich stehen die Arbeiter in der mechanisierten industriellen Produktion im wörtlichen Sinn zwischen den Maschinen und Anlagen. Diese Lage macht ihnen den Überblick über den gesamten Produktionsprozess unmöglich, die strategischen Posten haben die Vorgesetzten inne. Mit der Automatisierung der Produktion ändert sich diese Position, die Arbeiter stehen jetzt „neben, vor, über dem Prozess“ (Haug et al. 1980:87).

„'Früher stand an jedem Regler ein Mann.' Der Arbeiter hatte hier die Funktion des Reglers einer oder weniger Prozeßvariablen innerhalb vorgegebener Grenzwerte und tat damit routinemäßige Kopfarbeit: ständige Beobachtung weniger Meßwerte und regulierende Eingriffe, ohne die Ursachen der Abweichungen begreifen zu müssen. Konnte er durch seinen Eingriff Abweichungen nicht kompensieren, sprang der immer anwesende Meister oder Schichtführer herbei, um rasch zu beurteilen, was zu tun notwendig war.“ (Haug et al. 1980:86)

Die Entwicklung von einheitlichen pneumatischen und elektrischen Signalsystemen sowie den Mitteln zur Signalübertragung erlaubt es, die Fernsteuerungs- und Fernüberwachungsfunktionen zu bündeln, und die Messwarte entsteht als neuer, zentraler Arbeitsplatz jenseits der Anlagen. Für die Arbeiter impliziert dies eine neue Qualität der Arbeitsteilung innerhalb der Produktionsmannschaften, die sich in lokale und zentralisierte Anla-

\footnotetext{
${ }^{74}$ Eine weitere Möglichkeit zur Steigerung der Motivation ist die Einführung einer Gewinnbeteiligung, wie sie bereits 1871 bei den Farbwerken Höchst erfolgt (Bäumler 1963:273). Darüber hinaus wurde, um die hohen Fluktuationsraten zu senken, beispielsweise um 1900 bei Bayer eine „Dienstalterprämie“ gezahlt (Peetz 1981:157).
} 
genüberwachung bzw. Anlagenfahrer und Messwarte differenziert. In der Messwarte entsteht eine neue Arbeitsform, die als „Arbeit sui generis“ zu charakterisieren ist (Kern, Schumann 1985:149). Der Messwart arbeitet in einem von den Anlagen getrennten Raum und steuert den Produktionsprozess ausschließlich auf der Grundlage von Sekundärinformationen, die ihm entweder die Symbole vermitteln oder aber von den Anlagenfahrern aus den Produktionsräumen übermittelt werden. Sinnliche Wahrnehmung durch Geruch, Temperatur oder Lautstärke entfallen (Böhle, Rose 1992). Diese Form der Mediatisierung und räumlichen Trennung machen die Messwarte zu einem „neuartigen, auf abstrakte Zeichen reduzierten Wahrnehmungsraum“ (Tacke 1997:151). Der Arbeiter wartet innerhalb des ihn umgebenden panoramaartigen Symbolsystems nicht ab, bis eine Störung entsteht, sondern erkennt Prozessstörungen im Ansatz. Eine wesentliche Komponente wird somit das Erfahrungswissen, verstanden als umfassende Kenntnis aller optischen Signale im Normalzustand, die es erlaubt, selbst kleinste Abweichungen als optisch veränderte Zeichenkonstellation differenziert wahrzunehmen (Mickler et al. 1975). Die lokale Anlagenüberwachung wird zu einem wesentlichen Komplement dieser Messwartentätigkeit, denn erst der regelmäßige Informationsaustausch und die Verbindung von dezentraler und zentraler Perspektive erlaubt eine gesicherte Aussage über den Prozesszustand. Kooperation und Kommunikation sind damit unverzichtbarer Bestandteil der Arbeit auf dem Shop floor. Als Grundfunktionen der lebendigen Arbeit an diesen teilautomatisierten Großanlagen identifizieren Kern und Schumann „Programmfixierung, Prozesskontrolle, Prozessregulierung und Instandhaltung“ (1990:240), wobei für die Beschäftigten die Prozesskontrolle und - regulierung als Hauptaufgaben verbleiben. Die Arbeit an modernen Prozessleitsystemen ist zunehmend Arbeit an Bildschirmplätzen (Tacke 1997). Bildschirme, Tastaturen, Drucker und Lichtgriffel ersetzen traditionelle Analoginstrumente, Abstraktionsfähigkeit und Systemdenken müssen inhärenter Bestandteil des Arbeitshandelns werden.

Industriesoziologische Studien ${ }^{75}$ bilanzieren diesen Automatisierungsprozess und seine Folgen für die Arbeitssituation vorwiegend positiv und zwar sowohl in der Regulations -, der Qualifikations- wie auch der Belastungsperspektive. Die technischen Veränderungen im stoffumwandelnden Bereich hätten im allgemeinen keine negativen Auswirkungen auf die Arbeitssphäre, durchweg sei sogar ein Abbau restriktiver Arbeitselemente festzustellen. (Kern, Schumann 1990:257) Für den Ist-Zustand der frühen 1980er Jahre konstatieren sie:

„Nach unseren Beobachtungen ist moderne Chemiearbeit so sonderlich anstrengend nicht - gegenüber der extrem aufreibenden Arbeit in der Chemiefabrik früherer Tage ein ins Auge stechender Kontrast.“ (ebd. 258)

Die positive Einschätzung resultiert hauptsächlich aus einer veränderten Belastungssituation der Chemiearbeiter. Es minimieren sich die ursprünglich den Charakter der Arbeit im Chemiebetrieb prägenden Belastungen wie Lärm, Hitze und körperliche Anstrengungen. Die Technisierung bringt indessen andere belastende Faktoren hervor, wie etwa psychisch - kognitive und psychisch - affektive Anforderungen, die aus dem neuen Verantwortungsdruck sowie dem Wechsel des Arbeitsrhythmus entstehen: Anstrengende Reizüberflutung während der Störungsphasen und Reizarmut im Normalbetrieb der Anlage wech-

\footnotetext{
${ }^{75}$ Vgl. Blauner 1964; Fürstenberg 1969; Kern, Schumann 1970; 1984.
} 
seln sich unkalkulierbar ab. So sehen Mickler et al. (1975:401ff.) für den körperlichen Aspekt sogar eine gewisse Unterforderung durch mangelnde „muskuläre Beanspruchung“, betonen aber, dass eine hohe psychische Belastung der Beschäftigten aus dem unvorhersehbaren Wechsel zwischen dieser Unterforderung bei normalem Prozessverlauf und Überforderung bei Störfällen resultiert.

Festzuhalten ist, dass die Technisierung der Chemieproduktion, die mit der Einführung der Mess- und Regelsysteme begann, nicht nur Arbeit durch verstärkten Technikeinsatz substituiert, sie transformiert sie darüber hinaus in erheblichem Maß. Planende, steuernde und kontrollierende Merkmale der Arbeit an auf kontinuierlich produzierenden, hocheffizienten Apparaturen lösen den direkten Herstellungsbezug der Arbeit ab. Dieser neue Arbeitstyp, der in der Industriesoziologie als Systemregulierung bezeichnet wird (Schumann et al. 1994), beruht einmal auf einem quantitativen, aber auch qualitativen Bedeutungszuwachs sekundärer Tätigkeiten der Prozessvorbereitung und Prozessregulierung (Qualitätssicherung, Instandhaltung, teilweise sogar Optimierung und Planung).

Die Kernfunktionen dieser „mediatisierten Produktionsarbeit“, aus denen sich Systemregulierung zusammensetzt, sind im Vergleich zur Handarbeit ${ }^{76}$ oder Maschinen- und Anlagenführung ${ }^{77}$ äußerst komplex. Im Zentrum steht das Regulieren, das alle mittelbar produktiven Tätigkeiten bezeichnet, die einen vorübergehenden Eingriff in den Programmablauf umfassen mit dem Ziel, bei einer Störung den Normallauf wieder herzustellen oder bei Abweichungen den Normallauf zur Sicherung des Produktionsergebnisses zu verlassen und eine direkte Steuerung der Prozesse vorzunehmen. Neben diesen aktiven Eingriffen umfasst Regulieren aber auch das kontinuierliche Beobachten und Kontrollieren der Prozesse, um einen Abgleich zwischen Normallauf und Störung vornehmen zu können. Fehlersuche und Störanalyse gehören damit zum Tätigkeitsprofil der Systemregulierer, insgesamt kann Regulieren sich aber auf ganz unterschiedlichen qualifikatorischen Niveaus bewegen und durch die Anreicherung von Prozessoptimierung, Reparieren, Warten, Instandsetzen, Einrichten und Rüsten sowie Prüfen und Qualitätssicherung aufgewertet werden.

Die Messwartentätigkeit verlangt zusammenfassend fachliche Kompetenzen, die Kenntnisse über die Geografie, die Wirkungsweise sowie die Instrumentierung der Anlage umfasst. Einen hohen Stellenwert im Arbeitshandeln hat das Erfahrungswissen, denn erst langjähriger Umgang mit der Anlage und dem Prozess erlaubt den Arbeitskräften eine gewissenhafte und eigenständige Durchführung vorbeugender Interventionen. Eine detaillierte, theoretisch fundierte Kenntnis der Funktions- und Konstruktionsweise der Anlage sowie der Reaktionsabläufe ist hingegen nicht erforderlich.

\footnotetext{
76 „Handarbeit am Produkt umfasst in der Chemischen Industrie überwiegend Verpackungs- oder Palettiertätigkeiten. Der Tätigkeitstyp Handarbeit an Maschinen/Anlagen umfasst in der Chemische Industrie in erster Linie manuelle Tätigkeiten wie das Absacken von Granulaten oder das Abfüllen von Flüssigstoffen. Auch Arbeiten wie das Ausschaufeln von Rückständen direkt oder innerhalb der Anlagen (...) zählen zu diesem Tätigkeitstypus.“ (Schumann et al.1994:576).

77 „Die Maschinen- und Anlagenführung umfasst Tätigkeiten bzw. Prozesseingriffe, die im Rahmen diskontinuierlicher Produktionsverfahren notwendig werden. Da in diesen Bereichen von einem breiten Einsatz gehobener Rezeptsteuerungen noch nicht die Rede sein kann (...), sind weiterhin ständige - manuelle - Prozesseingriffe notwendig, um den nächsten Verfahrensschritt auszulösen. Die Anlagen bzw. Steuerungen benötigen also der permanenten Führung durch die Beschäftigten.“(vgl. Schumann et al. 1994:575).
} 
„Die Arbeit ist eine recht komplexe Angelerntentätigkeit, aber auch sie erfordert keinen theoretisch vorgebildeten Facharbeiter oder gar Quasi-Techniker.“ (Kern, Schumann 1970:144)

Besonders deutlich wird diese durchaus widersprüchliche Zusammensetzung des Anforderungsprofils etwa daran, dass es in Chemiebetrieben nicht üblich ist, Aushilfskräfte für wenige Wochen einzustellen, wie dies beispielsweise in der Automobilindustrie in Teilen der manuellen Montage getan wird. Denn obgleich Chemiearbeit allen Technisierungsverläufen zum Trotz eine Angelerntentätigkeit bleibt, so hat sie doch ihren Charakter als „Jedermannarbeit“ durch die neuen Anforderungen in den technisierten Betrieben verloren.

\subsubsection{Ausbildung und Kompetenzprofile}

Die Ausgangssituation und Entwicklung in der Chemischen Industrie im bezug auf die Arbeitskräfte unterscheidet sich deutlich von anderen industriellen Branchen, vor allem der Fertigungsindustrien, da es zu keiner Zeit ein „Chemie - Handwerk“ gibt. Die Konstituierung der Chemischen Industrie erfolgt über die Modifikation der theoretischen Wissenschaft hin zu einer „angewandten Wissenschaft“, ohne dass auf dem Weg zur industriellen Massenproduktion Handwerkstraditionen gefährdet und Arbeitskräfte massenhaft dequalifiziert wurden. Produktionsarbeiter in der Chemischen Industrie waren wenig qualifizierte ,Massenarbeiter ${ }^{6}$, sie wurden nicht erst durch Taylorisierung dazu gemacht (Schumann et al. 1994). Im Zuge der Ausweitung und Kontinuisierung der Produktion chemischer Stoffe wandeln sich die für den Einsatz in chemischen Betrieben notwendigen Qualifikationen und damit korrespondierend die betrieblichen Aus- und Weiterbildungsstrategien.

Bis Mitte der 1930er Jahre greifen die Unternehmen bei der Rekrutierung von Arbeitskräften auf Ungelernte bzw. fachfremd Qualifizierte zurück und müssen dies auch. Eine spezifische Berufsausbildung für Chemiearbeiter gibt es nicht, und aus Sicht der Unternehmer ist dies kein großes Manko, denn „,in der Regel ist jeder normal entwickelte und gesunde Mensch zur Arbeit in der Chemischen Industrie geeignet“ (Handbuch der Berufe 1933:24). Mit dem technischen Niveau verstärkt sich aber der innerbetriebliche Unterweisungsaufwand. In der Praxis bedeutete dies, dass die Arbeiter in die Betriebe kommen, ohne die Produktionsabläufe nachvollziehen oder sich mit ihren Produkten identifizieren zu können, und dass die ebenfalls nicht einschlägig ausgebildeten Beschäftigten zunehmend Schwierigkeiten beim Anlernen haben. Kompetenzdefizite beziehen sich vor allem auf die Merkmale „chemisches Grundlagenwissen“, „Branchen- und Firmenkenntnis“ und „abteilungsspezifische Berufserfahrung“ (Christ 1995). Dennoch halten die Chemieunternehmen an ihrer traditionellen Praxis fest und belassen es dabei, die Kenntnisse und Qualifikationen in meist mehrjährigen, von Produktionsstätte zu Produktionsstätte divergierenden Praktiken des Anlernens zu vermitteln. In Ermangelung fehlender staatlicher oder auch unternehmensinterner Regulierungen und Formalisierungen liegt die Beschäftigtenqualifizierung in der Verantwortung der leitenden Chemiker.

„Da sich der Gesamtbetrieb im Grunde aus ,Kleinbetrieben’ zusammensetzte, lag es nahe, die Ausbildung des Chemiefacharbeiters auch ,kleinbetrieblich' auszugestalten. Anstelle einer organisierten Ausbildung mittels Ausbildungsplänen und -richtlinien stand die per- 
sonengebundene, unstrukturierte und nur der aktuellen Bedarfslage angepasste Qualifizierung im Vordergrund. [...] Die für die Ausbildung verantwortlichen Chemiker realisierten nämlich vor Ort mehr oder weniger ausgereifte ,bildungspolitische Insellösungen', unterließen es aber, konsequent eine übergreifende, einheitliche und systematische Ausbildung anzustreben.“(Christ 1995:233)

Bis zur zweiten Hälfte des Jahres 1938, also beinah 70 Jahre, gibt es trotz der kontinuierlichen Ausweitung und des damit verbundenen Beschäftigtenanstiegs in der chemischen Industrie keine spezifischen Berufe in diesem Industriezweig. Einzige Ausnahme bilden die wenigen Chemieschulen, die den Bedarf an analytisch ausgebildeten Hilfskräften für die Laboratorien decken. Ein Grund für diese fehlende „Professionalisierung“ könnte in dem rasanten Tempo der Entwicklungen liegen. Die Ansätze für die Herausbildung spezifischer Berufsprofile sind meist obsolet, bevor sie überhaupt in Schriftform festgehalten werden können. Die einzelnen Etappen der chemischen Forschung und Produktion folgen so dicht aufeinander, dass ein klares Anforderungsprofil, dass dem Berufsbild zugrunde liegen könnte, nicht formuliert werden kann (Mühlbauer 1961). Eine andere Erklärung für eine ausbleibende Verberuflichung liegt in dem anhaltend niedrigen Niveau der Arbeitsanforderungen.

1938 schließlich wird die erste planmäßige Ausbildung mit Abschlussprüfung zum Chemiejungwerker erlassen, ${ }^{78}$ doch nach wie vor bleibt Chemiearbeit ein Anlernberuf und in der Praxis die berufliche Erstausbildung bzw. ein einschlägiger Berufsabschluss ohne Bedeutung. ${ }^{79}$ Der Ausbildungsberuf Chemiefacharbeiter entsteht erst in den unmittelbaren Nachkriegsjahren und wird am 30. Juni 1949 als offizieller Ausbildungsberuf von staatlicher Seite anerkannt. ${ }^{80}$ Diese Entscheidung kann zum einen als Reaktion auf die Klagen und wachsenden Schwierigkeiten der Unternehmen im Hinblick auf nicht ausreichend qualifizierte Beschäftigte gelesen werden. Zum anderen spielt auch die „berufsethische“ Seite eine Rolle. Wo in anderen Bereichen der industriellen Produktion die Arbeiter eine Art „Berufsstolz“ mit in die Betriebe bringen, entfällt dies für die angelernten oder branchenfremd ausgebildeten Arbeitskräfte in der Chemie. Eine geregelte Ausbildung mit einem Facharbeiter - Abschluss soll dieses Manko beheben und die Attraktivität der Chemiearbeit heben (Drexel 1979). Allgemein verbindliche Inhalte oder ein schlüssiges Konzept des neuen Ausbildungsganges liegen freilich zu dieser Zeit nicht vor und es dauert fast zehn Jahre, bis schließlich 1958 die Ausbildungsinhalte sowie ein entsprechendes Berufsbild entwickelt sind. Trotzdem bleibt die Zahl der Ausbildungsverhältnisse bis in die 1970er Jahre hinein gerade vor dem Hintergrund des stetig hohen Beschäftigungswachstums der Branche hinter den Erwartungen zurück, einzig im Bereich der Erwachsenenbildung wird das neue Berufsbild forcierter in Anspruch genommen.

\footnotetext{
${ }^{78}$ Mitteilung der Anerkennung durch RI vom 08.11.1938 - IX-42 000 / 6 [R 11 / 928 / 62].

${ }^{79} \mathrm{Ob}$ dies an den besonderen Bedingungen der Kriegswirtschaft oder aber an der unzureichenden, da zu schmalen Konzeption des Qualifikationsprofils lag, ist historisch nicht nachzuzeichnen (Drexel 1979; Christ 1995).

${ }^{80}$ Der Chemiefacharbeiter entstand im Rahmen eines 1949 vom Arbeitsring der Arbeitgeberverbände der Deutschen Chemischen Industrie e.V., der Arbeitsstelle für Betriebliche Berufsausbildung in Bonn, der Industrie- und Handelskammern sowie Vertretern der Arbeitnehmerschaft ausgearbeiteten Gesamtpakets „naturwissenschaftlich orientierter Berufe“, zu denen der Chemie- (1949), Physik- (1951), Lack- (1952), und Biologielaborant (1956), Kunststoff- (1949) und Bleischlosser (1955) sowie der Meß- und Regelmechaniker (1960) gehörten.
} 
Die Zurückhaltung der Großunternehmen erklärt sich unter anderem aus der Organisationsstruktur der Chemischen Industrie: Obgleich in den zentralen Personalbereichen die Grundsatzentscheidung für eine Facharbeiterausbildung fällt, ist den Betriebsleitern vor Ort unklar, wie sich das neue Qualifikationsprofil in der Praxis bewähren soll. Erst nach Jahren, in denen die Ausbildung von persönlichem Engagement und persönlichen Idiosynkrasien engagierter Befürworter getragen und geformt wird, bildete sich allmählich ein spezifisches Qualifikationsprofil heraus. (vgl. ebd.)

Hemmend auf die Durchsetzung des neuen Chemiefacharbeiters wirkt außerdem das traditionelle Rationalisierungsverständnis der Branche. In der Umsetzung der technischen Innovationen werden komplexe, kapitalintensive Produktionsanlagen aufgebaut, die in ihrer Anfahrphase nicht nur schnell, sondern auch sicher in Gang gebracht und dann kontinuierlich und möglichst störungsfrei in Betrieb gehalten werden müssen. Sowohl die personelle Besetzung der Anlagen, also die Belegschaftsgröße und Qualifikationsstruktur, als auch die Arbeitsorganisation werden von diesen Überlegungen unter der Prämisse einer möglichst kostengünstigen Produktion zu einem nicht unerheblichen Anteil geleitet.

„Unter diesen Umständen konnte das Rentabilitätsprinzip bei der Gestaltung der Arbeitsorganisation nur in der Vermittlung zwischen den notwendigen widersprüchlichen Momenten einer Minimierung der Personalkosten durch Reduktion von Belegschaftsgrößen und Ausbildungsaufwendungen einerseits sowie der Gewährleistung hoher Produktionsleistung durch eine ausreichend große und qualifizierte Anlagenbesatzung andererseits liegen.“ (Mickler et al. 1975:457)

Gerade für den Neustart von Produktionsanlagen oder Anlagenteilen bedeutet dies in den meisten Betrieben eine Entscheidung für eine recht hohe Belegschaftsstärke, wobei allerdings ein Großteil der höheren Qualifikationsanforderungen auf wenige Arbeitsplätze beschränkt ist und die Mehrheit der Beschäftigten auf gering qualifizierten Arbeitsplätzen eingesetzt wird. Vor allem die zentrale Messwarte gehört zu den qualifikatorisch anspruchsvollen Plätzen, und die Betriebsleitung besetzt diese mit wenigen prozesserfahrenen Arbeitern. ${ }^{81}$ Das Unternehmen kann so mit einer an sich nur unzureichend eingearbeiteten Produktionsmannschaft das Maximum an Prozesssicherheit bei - trotz einer hohen, aber vor allem in den unteren Lohngruppen eingestuften Beschäftigtenzahl - niedrigen Personalkosten erreichen. In der Phase der Routinisierung der Produktion verliert der Sicherheitsaspekt im unternehmerischen Kalkül zunehmend an Brisanz, so dass die Betriebe Rationalisierung nun eher über arbeitsorganisatorische Maßnahmen zur Verringerung der Personaldecke betreiben. Die Arbeitsbereiche pro Beschäftigten werden ausgeweitet, vor allem durch Anlagerung einfacherer Labor- und Wartungsfunktionen. Die partielle Aufhebung der Spezialisierung und die Flexibilisierung der Prozessarbeiter hat dann zur Folge, dass der für den Neustart eingesetzte personelle Überhang sukzessive abgebaut wird. Die aus dieser Minimierung resultierende Aufqualifizierung der Anlagenfahrer bleibt aber auf die ohnehin vergleichsweise niedrigqualifizierten Arbeitsfunktionen im anlagennahen Bereich beschränkt und erreicht in keinem Fall das hohe Niveau der Messwartenarbeit (Mickler 1975).

\footnotetext{
${ }^{81}$ Auch hier wirkt der Primat des Erfahrungswissens, denn rekrutiert werden diese Fachkräfte mehrheitlich aus Betrieben, die auf ähnlichen Prozessen basieren.
} 
Erst Mitte der 1970er Jahre ist trotzdem eine deutliche Zunahme der Ausbildungsverhältnisse zu verzeichnen, die einerseits sicherlich auf die Entscheidung der Großchemie, die Ausbildungskapazitäten auszuweiten, zurückzuführen ist. Vor allem ist sie aber auch Ausdruck der deutlich angespannteren Lage auf dem Ausbildungsmarkt. Die allgemeine Lehrstellenknappheit macht selbst die Bereiche, die als nicht sonderlich attraktiv, da nicht voll etabliert gelten, für eine höhere Anzahl an Jugendlichen interessant, und insbesondere die Ausbildung zum Chemikanten gewinnt im Verhältnis zu anderen Ausbildungsberufen an Relevanz.

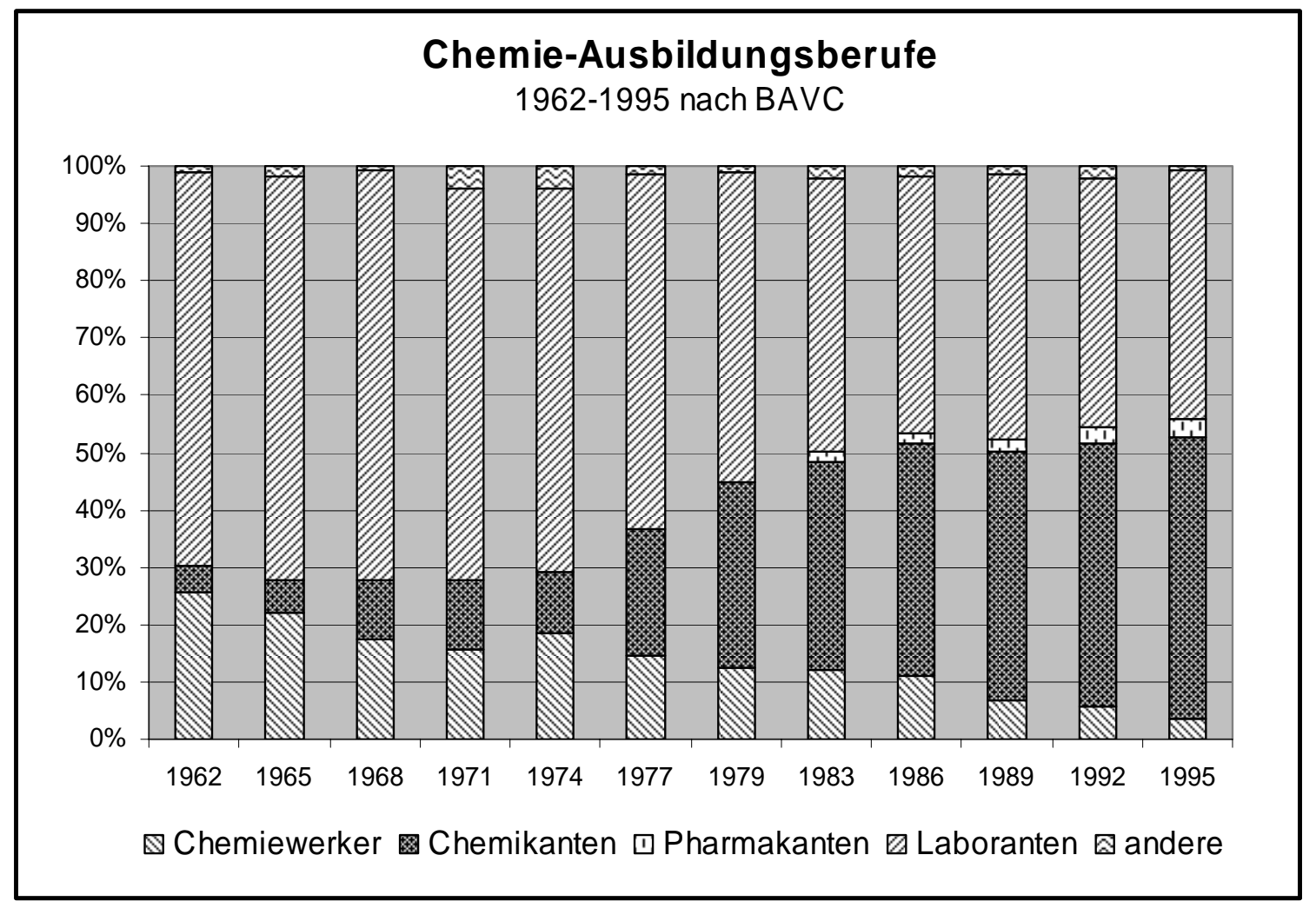

Abbildung 2: Entwicklung der Chemie-Ausbildungsberufe; BAVC (1996)

Die genauen Beweggründe für die personalpolitische Grundsatzentscheidung in der Chemischen Industrie zur Expansion der Ausbildung ist für die 1970er und 1980er Jahre nicht eindeutig zu beantworten. Drexel (1982) kommt zu dem Schluss, dass es innerbetriebliche technisch-ökonomische Veränderungen sind, die die Unternehmen zu diesem Strategiewechsel veranlassen: Die relative Personaldichte hatte sich so massiv verringert, dass ein selbsttätiger Erwerb von Qualifikationen im Arbeitsprozess, wie ihn die klassische Angelerntenkarriere in der Chemie vorsah, immer weiter eingeschränkt werde. Damit sei eine verstärkte Förderung der chemiespezifischen Ausbildung notwendig. Darüber hinaus macht Drexel gesellschaftliche Veränderungen für ein Einschwenken der Branche auf die Facharbeiterausbildung verantwortlich. Die Betriebe bekämen immer größere Schwierigkeiten, handwerklich vorgebildete Arbeitskräfte zu rekrutieren. Dieses sei vor allem ein Problem im Hinblick auf „berufsethische“ Qualifikationen wie Disziplin, Fleiß und Gehorsam. Allein der technische Wandel sowie die dominanten Arbeitsstrukturen der Chemischen Industrie stellten noch keinen Zwang zu einer solchen Politik dar. Dies belegen 
die Ergebnisse von Kern und Schumann (1990). Sie resümieren, dass diese Politik vor allem das

„Einschwenken auf das Modell ,Personalminimierung durch Funktionsverschmelzung und Qualifizierung’ erleichtert.“ (ebd. 242)

Sie sehen in der Chemischen Industrie - wie in anderen Branchen auch - Ansätze zur Realisierung eines Neuen Produktionskonzepts, welches das alte Prinzip der „Organisationsform strenger Arbeitsteilung“ (Mickler et al. 1975) aufweiche. Die in der Produktionsarbeit verbleibenden Prozesse der Kontrolle und Regulierung würden in diesem Konzept je nach Bedarf festgelegt und kurzfristig im Zuständigkeitsbereich des Gesamtarbeitsplatzes verändert. Das Auflockern der Arbeitsteilung durch Neue Produktionskonzepte bleibt auf einen engen Raum - Produktionsmannschaften in einigen Bereichen, und auch da nur zögerlich - beschränkt. Die interne Flexibilisierung der Produktionsmannschaften wird von den Unternehmensleitungen zwar durchaus positiv bewertet, aber nicht systematisch in eine entsprechende Gestaltung der Arbeitsorganisation umgesetzt. So bleibt die Schnittstelle zwischen Produktion und Instandhaltung bestehen, und auch eine vertikale Aufgabenintegration (Meister/Schicht) steht nicht zur Debatte.

„Den Weg zur Anreicherung der Produktionsarbeit durch Verlagerung dispositiver Funktionen nach unten wird die Chemische Industrie mit Sicherheit nicht gehen, und sie könnte es vielleicht auch nicht.“ (Kern, Schumann 1990: 269)

Die Autoren sehen für zukünftige Rationalisierung in der Chemie eher eine Anreicherung der Produktionsarbeiter-Tätigkeiten durch Verlagerung routinemäßiger Wartungs- und Reparaturaufgaben. Dieses sei im Rahmen der höheren fachlichen und beruflichen Qualifikation der Beschäftigten eine sinnvolle und vor allem auch notwendige Funktionsintegration, denn

„in dem Maße, in dem nun ausgebildete Facharbeiter in den Betrieben auftauchen, muß man ihnen aber auch - will man Friktionen vermeiden - facharbeitergerechte Arbeitsplätze anbieten. Ohne eine solche Fundierung des Chemiefacharbeiters in den Arbeitsstrukturen würde der Abschluß in der Luft hängen.“ (Kern, Schumann 1990: 267)

Die Ökologiedebatte sowie eine Reihe von Chemieunfällen bilden letztlich seit Mitte der 1970er Jahre einen nicht zu unterschätzenden äußeren Druck zur Ausbildung und Einstellung von Chemiefacharbeitern. 1986 findet im Rahmen der Neuordnung der naturwissenschaftlichen Berufe eine Modifikation des Berufsbildes des Chemiefacharbeiters statt. Inhaltlich sind es vor allem Umweltschutz- und Energiesparaspekte, die aufgenommen und während der gesamten Ausbildungszeit vermittelt werden sollen. Darüber hinaus werden auch mikrobiologische Arbeitstechniken, Aufgaben aus dem Bereich der Installation und Prozessleittechnik sowie der Informationstechnik in den Rahmenplan aufgenommen.

Zu einer systematischen Modifikation interner arbeitsorganisatorischer Modelle kommt es durch diese Neuordnung allerdings nicht, so dass die betrieblichen Arbeitsplatzerfordernisse hinter den vorhandenen Qualifikationen, die die Beschäftigten nun zunehmend mit in den Betrieb bringen, zurückbleiben. Viele Großunternehmen sehen sich daher trotz einer anhaltenden Arbeitsmarkt- und Ausbildungskrise mit wachsenden Schwierigkeiten konfrontiert, Jugendliche für die Ausbildung in der Chemischen Industrie zu gewinnen. 
Diese Zurückhaltung wird von Kern und Schumann vor allem auf die Unsicherheit der Jugendlichen über das Berufsbild des Chemiefacharbeiters zurückgeführt. ${ }^{82}$

„Die Kategorie des ,Berufs' ist auch in dieser Gruppe besetzt mit dem Bild des klassischen Handwerkers und Facharbeiters: der etwas weiß und kann, dessen Fachkompetenz sich umsetzt in ein ,Können', das ihm in seiner Ausbildung vermittelt wurde und das sich in spezifischer Leistung vergegenständlicht.“ (Kern, Schumann 1990:277)

Im betrieblichen Alltag wird das ohnehin fragile Berufsverständnis in mehrfacher Hinsicht unterminiert (Kern, Schumann 1990). Einmal spielen theoretische Qualifikationen in der Arbeitspraxis kaum eine Rolle. Dies bedeutet konkret, dass die Chemikanten in den Betrieben trotz ihrer guten Ausbildung zunächst ,gar nichts' können, fachlich also dem erfahrenen Ungelernten unterlegen sind. Auch nach der Einarbeitungszeit bringen die theoretischen Kenntnisse keine strategischen Vorteile und werden in der Regel schnell wieder vergessen. Die einmal erlernte beruflich-theoretische Kompetenz geht so verloren. Chemiefacharbeiter können ein Bewusstsein um ihr spezifisches Können, dass zunächst vor allem auf theoretischen Fachkompetenzen beruht, in der betrieblichen Praxis kaum weiterentwickeln. Ihre „Arbeitsleistung“ ist kaum zu identifizieren, denn die weitgehende Automatisierung der Anlagen lässt kaum Raum für eigenes, unverwechselbares Können. Chemiefacharbeit ist also mit einem Leistungsbegriff verbunden, der sich negativ über das Nicht-Eintreten von Störungen definiert (Kühnlein 1990). Insgesamt ist „der Funktionszuschnitt des Anlagenfahrers [...] einfach [...] zu schmal, als dass er einem Berufsbild entsprechen könnte.“ (Kern, Schumann 1990:278).

$\mathrm{Zu}$ dieser fachlich enttäuschenden Situation treten die für die jungen Chemiefacharbeiter prekären Milieubedingungen des traditionellen Angelerntenbetriebs. Denn nach wie vor müssen die Unternehmen aufgrund der nur zögerlich steigenden Ausbildungszahlen auf Ungelernte zurückgreifen. Als Neuling bereits in der höchsten Lohngruppe eingestuft, werden sie täglich damit konfrontiert, trotz ihrer fundierten Kenntnisse ihre Kompetenzen nur eingeschränkt abgefragt werden und sie in der betrieblichen Realität kaum besser zurecht kommen, als die Ungelernten.

„Wie bei der Arbeitsausübung die ,Alten’ zunächst die eindeutig Überlegenen sind, und der Chemiefacharbeiter der Anfänger ist, kann hier manche Ranküne ausgetragen und mancher Erfahrungstransfer blockiert werden.“ (Kern, Schumann 1990:279)

Vor allem für die Ausbildung einer berufsspezifischen Identität ist dies abträglich und kann zu mancher Irritation führen (Paul-Kohlhoff 1989).

\footnotetext{
${ }^{82}$ Sowohl Drexel, Nuber als auch Kern, Schumann berücksichtigen bei ihren Überlegungen nicht das gesellschaftliche Ansehen des Berufs seit Mitte der 1970er Jahre: „Konnten die von ihr (der Chemischen Industrie, K.B.) Beschäftigten früher wie selbstverständlich voraussetzen, gemeinhin als Produzenten sozial nützlicher Produkte angesehen zu werden, die den Menschen ein besseres und leichteres Leben ermöglichen, und lag hierin für die Chemieproduzenten auch eine Quelle der Identifikation und der sozialen Anerkennung über die eigene Arbeit, so hat sich ihre Situation inzwischen fundamental gewandelt: Das, was früher ausschließlich den Status von ,Nebenfolgen' und ,Randbedingungen' hatte - die negativen Folgen von chemischem Produkt und Produktionsprozeß für die natürliche Umwelt und für die Gesundheit der Menschen - ist inzwischen zu einem (für viele zu dem ) zentralen Bewertungskriterium geworden, mit der die ökologisch-kritische Öffentlichkeit an die Chemische Industrie herantritt und ihre Beschäftigten auf diese Weise dem Stigma aussetzt, an der Produktion von Umweltrisiken beteiligt, ja eine Gefahr für Mensch und Natur zu sein.“ (Heine, Mautz 1989:151) Dass diese sehr negative Einschätzung des Berufs einen erheblichen Einfluss auf seine Attraktivität hat, kann mit einiger Sicherheit vermutet werden.
} 
Eine erste Chance zur qualifikatorischen Aufwertung der Produktionsarbeit und einer damit verbundenen Neudefinition des Anforderungsprofils für die Chemikanten stellt die in den 1980er Jahren einsetzende Reorganisation der Instandhaltung in der Großchemie dar, die auf die Schnittstelle zwischen Produktion und Instandhaltung fokussiert: Meisterbereiche der Instandhaltung werden dezentralisiert und einzelnen Betrieben zugeordnet, Betriebsingenieure als Service-Leister an Produktionsbetriebe gegliedert. Eine Neuschneidung betriebsinterner Arbeitsstrukturen und Abläufe bleibt indes aus, und der weitaus größte Teil qualifikatorischer Entwicklungsanstöße, die sich aus der stetigen Verfahrensmodernisierung ergeben, wird jenseits der Position der Beschäftigten auf dem ShopFloor abgeschöpft. Einerseits geschieht dies durch die Vorortpräsenz von Chemikern, Ingenieuren, Betriebsmeistern und Automationsfachleuten, andererseits durch das Festhalten an der horizontalen und vertikalen Arbeitsteilung. Diese wird lediglich punktuell gelockert durch eine dezentrale Zusammenfassung problemlösender Knowhow Träger (Kern, Schumann 1990; Wöcherl 1989) in Form von Qualitätszirkeln und Fachteams.

Schließlich ist es die Technisierung, die perspektivisch eine Professionalisierung von Chemiearbeit durch eine Ausweitung von Arbeitsplätzen auf hohem Niveau von Systemregulierung bewirkt. Doch auch diese Entwicklung bringt nur für die Minderheit der Beschäftigten eine neue Berufsfachlichkeit. Das Anforderungsprofil für die in der Chemischen Industrie Beschäftigten schärft sich zwar an der technischen Entwicklung innerhalb der Produktion bzw. in direkter Auseinandersetzung mit dem Prozess, ohne dass es sich zwangsläufig auf Facharbeitsniveau einpendelt. Obwohl die technischen Voraussetzungen eine gute Grundlage für organisatorische und arbeitspolitische Innovationen bieten, setzt das Gros der Unternehmen nach wie vor auf expertenbasierte, technischwissenschaftszentrierte Innovations- und Rationalisierungsstrategien. So bescheinigen die Ergebnisse des Trendreport Rationalisierung der Chemischen Industrie einen im Vergleich zu den anderen untersuchten Branchen mit 47 Prozent hohen Durchsetzungsgrad der Tätigkeitsstrukturen des Systemregulierers, und finden diesen Vorzugsweise in den technisierten Bereichen. ${ }^{83}$ Obschon diese Ergebnisse auf den ersten Blick die These eines technisch induzierten Strukturwandels von Produktionsarbeit bestärken, belegen die Analysen, dass Technisierung eine notwendige, nicht hinreichende Voraussetzung für Systemregulierung ist. Denn wo sie nicht gekoppelt ist an organisatorische und arbeitspolitische Innovationen, werden professionellere Standards für Produktionsarbeit sich nicht durchsetzen können. Entscheidend für das Niveau ist, wie hoch die Prozess- und Eingriffskompetenzen der Beschäftigten sind bzw. wie diese vom Betrieb definiert sind. Systemregulierung versinnbildlicht so einerseits die seitens des Managements lange Zeit in Frage gestellte Anerkennung der Tatsache, dass ungeachtet der Verwissenschaftlichung und Modellierung der Produktionsprozesse Lücken der Prozessoptimierung, -steuerung und Gewährleistung fortwähren und Interventionen menschlichen Handelns technische Desiderate kompensieren müssen (Schumann et al. 1994). Den Umfang dieser Interventionen bestimmt indes nicht die Technik, sondern sie ist in besonderem Maß von arbeitsorganisatorischen Entscheidungen der Betriebsleitung abhängig.

\footnotetext{
83 „Im Gesamt der von uns untersuchten Betriebe stellt der Arbeitstyp des Systemregulierers heute im Automobilbau 8\%, im Werkzeugmaschinenbau 10\% und in der Chemischen Industrie 47\% der Produktionsarbeiter. Diese Anteile fallen ausschließlich in den jeweils besonders weitreichend technisierten Produktionsbereichen innerhalb der Industrie sehr viel höher aus (...)“ ( Schumann et al.1994:644).
} 
Dies unterstützt die Ergebnisse von Schumann et al., die drei Typen von Arbeitsorganisation unterscheiden, die sich unabhängig vom Technisierungsgrad der Einzelbetriebe herausbilden und folgenreich für das Profil der Systemregulierer sind (Schumann et al. 1994:600 ff.).

Betriebe mit traditionell-hierarchischer Arbeitsorganisation entsprechen dem geschilderten Angelerntenmodell, d.h. die etablierten Arbeitsteilungsmuster werden fortgeschrieben. In der Regel bleiben die Beschäftigten hier von komplexen Regulierungsaufgaben und Prozessentwicklungen ausgeschlossen. Bedingt durch die Technisierung treten neben die auf Handarbeit basierenden Tätigkeiten Aufgaben aus dem Bereich der Systemregulierung, die jedoch in der Organisationspraxis nur das Profil einer Systemregulierung mit stark eingeschränkter oder begrenzter Aufgabenzuweisung annehmen. In der Regel sind dies einfache Überwachungsaufgaben, routinisierbare Formen der Störbeseitigung und die Pflicht zur Information der „Regulierungsspezialisten“. Letztere sind häufig externe Beschäftigte aus den Automationsabteilungen, Meister oder Ingenieure, in seltenen Ausnahmen auch Kollegen aus der Schicht. Eingriffsbefugnisse sind, so überhaupt vorhanden, auf kleinschrittige Teilaspekte begrenzt. Die Beschäftigten sind reaktiv an die Prozesse gebunden und werden zum „Anhängsel“ der Anlagen mit wenig Spielraum für eigenständiges Handeln. Prekär wird diese Arbeitssituation insbesondere im Fall größerer Störungen, denn die mangelnde Prozesskenntnis lässt die Beschäftigten durch ihre qualifikatorisch wie organisatorisch eingeschränkten Interventionspotentiale so lange „ohnmächtig“, bis sie auf Anweisung des herbeigerufenen Vorgesetzten handeln dürfen.

Eine erste Auflösung hierarchischer Stufen und unterschiedlicher Regulierungsebenen wird im Typ des flexibel-teilintegrierten Organisationstyp erreicht. Durch Rotationsregelungen wird innerhalb der Schicht die vorhandene Funktionsmasse des Gesamtprozesses auf alle verteilt, d.h. es kommt auf dem Shop floor zu einer Aufgabenintegration, die das Tätigkeitsprofil auf dem Shop floor zur qualifizierten Systemregulierung aufwertet. Das mehr an Eingriffskompetenz manifestiert sich in der Regel daran, dass den Beschäftigten ein je unterschiedlicher „Fahrstil“ in der Messwarte seitens der Vorgesetzten informell erlaubt ist. Diese Prozessverantwortung ermöglicht es den Arbeitskräften, ein zumindest ansatzweise systematisches Verständnis der Prozesse zu entwickeln und erhöht ihre Chancen zur Anwendung, Entfaltung und Erweiterung von Kompetenzen und Kenntnissen des Produktionsprozesses. Aus zwei Gründen definieren Schumann et al. dieses Modell jedoch weiterhin als Angelernten-Modell: Erstens basiert der erweiterte Zugriff auf das Arbeitsvermögen der Beschäftigten auf informellen Regelungen und zweitens bleibt die bereichsübergreifende Kooperation etwa mit der Instandhaltung ausgeschlossen. Ungelernte haben in diesem Organisationsmodell zwar deutlich mehr Probleme bei der Prozessaneignung, dies ist freilich nicht der fehlenden einschlägigen Chemikantenausbildung geschuldet, sondern könnte durchaus noch mittels innerbetrieblicher Weiterbildung bewältigt werden.

Der flexibel-hochintegrierte Organisationstyp schließlich bedeutet eine echte Abkehr vom Angelernten-Modell und damit eine Chance für eine Aufwertung der Produktionsarbeit in Richtung Facharbeit für das Gros der Beschäftigten. Dominant ist in diesen Fällen ein Systemregulierer, dessen Tätigkeitszuschnitt als „,aufgewertet“ gilt: Seine Handlungssituation ist durch das Zusammenspiel komplexer und einfacher Regulierungshandlungen zur Sicherung wie Wiederherstellung der Prozessstabilität ebenso charakterisiert wie durch Prozessoptimierung. Diese Eingriffskompetenzen sind auf die gesamte Schicht ausgeweitet, die als „Produktionsteam“ alle Aufgaben offiziell eigenständig erfüllt. Die schichtinterne Hierarchie ist aufgelöst, und auch bereichsorganisatorische Grenzen werden durchlässiger. Die Kooperation mit Spezialisten und Instandhaltern zur Lösung programmtechnischer Anlagenprobleme gehört zum erweiterten Anforderungsprofil, die Beschäftigten in der Produktion werden als „Experten der Praxis“ zu gleichberechtigten Gesprächs- und Kooperationspartnern. Der Anteil an Chemikanten ist in diesen Fällen überdurchschnittlich hoch, und Schumann u.a. resü- 
mieren: „Die Prozessaneignung auf dem Weg eines ,learning by doing’, wie wir es bei den anderen Organisationsvarianten vorfanden, ist hier schlechterdings unmöglich.“ (Schumann et al. 1994:606)

Je nach unternehmerischer Schwerpunktsetzung sind ganz unterschiedliche Konsequenzen für die horizontale und vertikale Arbeitsteilung zu beobachten. Von einem generellen Trend zur Anhebung des Anforderungsprofils der Chemikanten kann nicht die Rede sein, und insbesondere in den Betrieben, die die technische Modernisierung personalpolitisch durch Akademisierung bewältigen, verschärfen sich in der Produktion die Ambivalenzen zwischen beruflichen Kompetenzen auf der einen und eingeschränkten Arbeitsfunktionen bei den Beschäftigten in der Produktion (Paul-Kohlhoff 1989)

Übersetzt in quantitative Verteilungen entsprechen Ende der 1980er Jahre nur ein Viertel der Betriebe dem flexibel-hochintegrierten Organisationstyp, die breite Mehrheit (54\%) bildet der Mischtyp und immerhin noch 21\% der Betriebe bleiben dem etablierten Modell verhaftet. Für die Gesamtheit der Chemischen Industrie bedeutet dies, dass etwa die Hälfte aller Arbeitsplätze von Systemregulierern Anforderungsprofile ausweisen, die unterhalb des Facharbeiterniveaus liegen. Die Produktionsintelligenz ist im Rahmen einer verwissenschaftlichen Produktion nicht gefragt, Prozess- und Verfahrensexperten bleiben die akademischen Kader der Entwicklungs- und technischen Abteilungen sowie die Betriebleitung. Die betriebliche Anerkennung der Beschäftigten in der Chemischen Industrie resultiert nicht aus dem Expertentum vermittelter und vermittelbarer Eigenheiten der jeweiligen Prozesse oder Verfahren, sondern beruht einzig auf den spezifischen, konkreten Erfahrungen mit der jeweiligen Anlage und den dort produzierten Stoffen. Das Anforderungsprofil von Chemiearbeit bleibt bis in die 1980er Jahre hinein für das Gros der Beschäftigten weit hinter ihren Kompetenzen als Chemiefacharbeiter zurück. Zwar wandelt sich die Branche von einer vom Anforderungsprofil des klassischen Massenarbeiters geprägten Industrie zu einer Produktionsfacharbeiterbranche, der Anteil von Systemregulierung auf (gehobenem) Facharbeiterniveau liegt immerhin bei 45\%. In ausgewählten Produktsparten kann dies allerdings bei gleichem technischen Niveau unterschiedliche qualifikatorische Anforderungen nach sich ziehen. Und nicht zu vergessen unterstreichen die Ergebnisse der Erhebungen auch, dass zu Beginn der 1990er Jahre bei steigendem Facharbeiteranteil mit 30\% Maschinen- und Anlagenführung, 4\% Handarbeit am Produkt und 21\% Handarbeit an Maschinen und Anlagen noch über die Hälfte der Tätigkeitsstrukturen nicht im Spektrum der Anforderungen von Systemregulierung zu verorten sind.

Dominantes Modell zur Aufbesserung des Status und einer Entfaltung beruflicher Interessen von Facharbeitern bleibt der Aufstieg innerhalb der Schicht vom Anlagenfahrer über die Position des Messwartes zum Kolonnen- und Schichtführer, und für die ambitioniertesten Chemikanten steht der Betriebsmeister zur Disposition. Doch gilt auch hier, dass in erster Linie ihr betriebliches Erfahrungswissen, ihre individuellen Tricks und Kniffe und nicht ihre Facharbeiterausbildung sie für die Aufstiegspositionen prädestinieren. Die Unternehmen können damit auf Basis einer immer besser ausgebildeten Belegschaft produzieren, diese Ausweitung des Facharbeiterpools schlägt sich im Rationalisierungs- und Innovationskalkül jedoch nur in homöopathischen Dosen nieder.

Diese Veränderungen sind nicht als Ergebnis einer strategischen Neuausrichtung der Rationalisierungspolitik in der Chemischen Industrie zu verstehen. Elemente dessen, was zu Beginn der 80er Jahre unter dem Label Neue Produktionskonzepte gefasst wurde, sind in 
der Chemischen Industrie zwar in weitaus größerem Umfang zu finden. Doch sind die Fälle eher selten, wo diese Elemente sich im Verständnis der handelnden Akteure zu einem arbeitsgestalterischen, unternehmensübergreifenden Gesamtentwurf zusammenfügen (Schumann et al. 1994). Das Verhältnis von Technisierung zur Arbeitsorganisation spielt auf Unternehmensebene bis Ende der 1980er Jahre keine große Rolle. Verstärkt wird dies durch die relative Unabhängigkeit der Einzelbetriebe bei der Abwicklung des operativen Geschäfts. Produktions- und steuerungstechnologische Optionen sowie Arbeits- und Personaleinsatzfragen werden im Rahmen des vorgegebenen Budgets autonom gewählt. Diese Tradition relativer Autonomie vieler Betriebe hemmt eine Vereinheitlichung von Konzepten oder auch nur eine „erfahrungsbasierten breiten Diskussion über Vor- und Nachteile verschiedener Ansätze“ erheblich (Schumann et al. 1994:553).

\subsection{Hierarchische Strukturen und Sozialgefüge}

Die aus der Wissensbasierung resultierende spezialistenzentrierte Zuordnung von Innovationskompetenz hat maßgeblichen Einfluss auf das Kooperations- und Statusmodell, d.h. die Formen vertikaler und horizontaler Arbeitsteilung sowie die dazu gehörigen „eigensinnigen“ sozialen Bezugssysteme (Jürgens, Lippert 1998) der Branche. ${ }^{84}$

\subsubsection{Interne Organisationsstruktur und Hierarchieebenen}

Die Aufbauorganisation der Großunternehmen in der Chemischen Industrie ist geprägt durch eine streng hierarchische Gliederung in Organisationseinheiten unterschiedlichen Umfangs, die bestimmt ist durch die Art, wie die Aufgaben, die zum Funktionieren eines Unternehmens erforderlich sind, auf einzelne Arbeitsbereiche und Stellen verteilt sind. Die Spezialisierung im Forschungs- und Produktionsbereich findet ihren Ausdruck in einer objektorientierten Aufbauorganisation der Chemieunternehmen. Die Organisation der Produktion gliedert sich in einzelne Produktsparten, innerhalb derer eine Vielzahl von Betrieben mit ähnlichem Produktspektrum zusammengefasst sind. Die Forschungs- und Entwicklungstätigkeiten bündeln sich parallel zu dieser Struktur in der Zentralforschung sowie in Technika und Versuchslaboratorien, in denen die Produktionsverfahren getestet werden. Ähnlich zentral organisiert sind Ingenieurwesen, Personalabteilung sowie die Energieversorgung.

Das Gros der deutschen Chemieunternehmen wird entsprechend diesem funktional hierarchisch integrierten Modell nach dem Zweiten Weltkrieg gegründet, und diese Organisationsform dominiert bis in die 1970er Jahre. Konkret bedeutete dies, dass in einem „System von Säulen“ jeder Säule eine Funktion (Produktion, Vertrieb, Forschung, Anwendungstechnik, Personalwesen u.s.w.) zugeschrieben wird. Die Wertschöpfungskette verläuft innerhalb dieser Struktur aus der Forschung über die Entwicklung in die Produktion. Kernaufgabe der Zentralforschung ist die Identifizierung, Bewertung und Validierung von Substanzen und Technologien, die eine potentielle Bedeutung für die einzelnen Geschäftsbereiche haben könnten. Zu diesem Zweck wird neben Eigenforschungsaktivitäten die akademische Forschung der Universitäten und Forschungseinrichtungen intensiv

\footnotetext{
${ }^{84}$ Die Darstellung bezieht sich, daran sei an dieser Stelle noch einmal erinnert, ausschließlich auf Großunternehmen. Für sie gilt ebenso wie für die Darstellung der Produktionsbetriebe, dass wir uns wissend um die Heterogenität der Branche auf Strukturmerkmale stützen, die alle Betrieb mehr oder weniger stark ausgeprägt aufweisen.
} 
verfolgt, um neues Wissen wenn nötig zu internalisieren, Einsatzgebiete zu definieren und intern gezielt Forschung auf die möglichen innovativen Produkte und Verfahren hin zu betreiben. Die Chemische Industrie kann dabei, wie wir gezeigt haben, auf eine etablierte Kultur der Kooperation zwischen Industrie und Akademie zurückgreifen. Eine herausragende Rolle spielen in diesem Prozess die Technischen Hochschulen. Hier ist es durchaus üblich, Lehrstühle mit Industrieforschern zu besetzen, um einen wechselseitigen Transfer von Know-how über direkte Kontakte sicher zu stellen. Da die bloße Vermittlung naturwissenschaftlichen Wissen oft genug nicht ausreicht, sondern die direkte Aneignung innovativer wissenschaftlicher Methoden zur weiteren Verwertung unabdingbar ist, sind diese personellen Transfers von hohem strategischen Wert.

Diese Form der Diffusion von tacit knowledge aus der Forschung in die Produktion setzt sich unternehmensintern fort. Eine wichtige Aufgabe der Zentralforschung besteht neben der Generierung von Innovationen und deren Umsetzung in Produkte in der Qualifizierung der von den Hochschulen in die Unternehmen eintretenden Chemiker und Ingenieure für die geschäftsfeldbezogene Arbeit in der Produktion, etwa als Betriebsleiter oder Betriebsingenieure. Absolventen fangen in der Regel in der Zentralforschung an, lernen das Unternehmen und die Geschäftsbereiche kennen und arbeiten in ersten Projekten mit. Nach etwa zwei bis drei Jahren wechseln die meisten Absolventen dann mit ihren Projekten in die Geschäftsbereiche, an die das Projekt vergeben wird. Dies kann dann entweder das Technikum sein oder aber der Betrieb, in dem der Newcomer als stellvertretender Betriebsleiter eingesetzt wird, um „sein“ Projekt weiter bis zur Produktreife zu bringen. Aus dieser Position wechselt das Gros dann als Führungskraft in einen ähnlichen Betrieb und übernimmt neben der wissenschaftlich-technischen Betreuung und Entwicklung der Produktion auch die betriebwirtschaftliche Verantwortung (Kädtler 1998). Diese Konstellation stellt - neben der Verbundtechnologie - eine zweite Besonderheit der deutschen Chemischen Industrie dar und sichert den Transfer von Wissen und langfristige Kooperationen nicht nur zwischen Geschäftsbereich und Zentralforschung, sondern auch in die Produktion hinein. Der beruflichen Sozialisation der Betriebsleitung kommt damit eine zentrale Bedeutung bei der Gestaltung der Schnittstelle zwischen Wissensgenerierung auf der einen und Produktentwicklung auf der anderen Seite zu. ${ }^{85}$

\footnotetext{
${ }^{85}$ Es sei an dieser Stelle noch einmal ausdrücklich darauf verwiesen, dass „Diffusion“ und „Wissenstransfer“ nicht gleichzusetzen sind mit einem linearen, aus der Forschung in die Produktion laufenden Prozess, sondern dass immer auch eine innerbetriebliche Rückkopplung stattfindet.
} 


\section{Typische Betriebsstruktur der Chemischen Industrie (alt)}
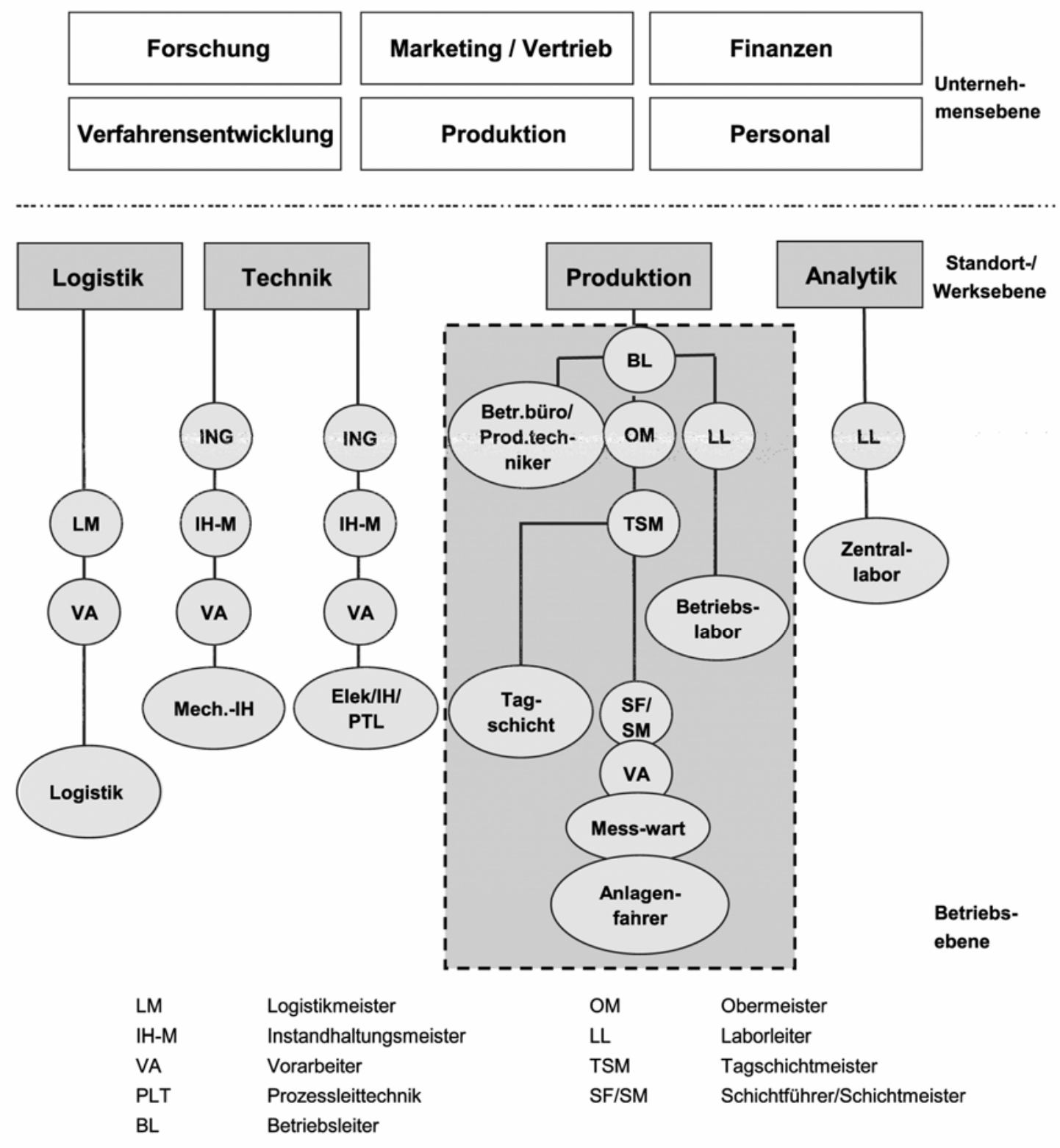

Abbildung 3: Typische Betriebsstruktur der Chemischen Industrie; SOFI (2003)

Die Betriebsleitung bildet die hierarchische Spitze der Produktionseinheit und setzt sich zusammen aus Ingenieuren, Technikern und Chemikern. Sie haben die Oberaufsicht und tragen die volle Verantwortung für alle betrieblichen Vorgänge. Organisatorisch gesehen stellen sie die Verbindung zum Unternehmen bzw. zum jeweiligen Ressort/Geschäftsbereich her. Ihre Leitungsaufgaben beziehen sich auf Personal, Anlagen, Produkt, Arbeitssicherheit und Umweltschutz, Produktion, Kommunikation sowie chemisch - technologische Änderungen und Weiterentwicklungen (Optimierung, Planung, Entwicklung). Die Produktpalette sowie die ökonomischen Betriebsziele werden von Ab- 
teilungs- bzw. Ressortleitern vorgegeben. Die Betriebsleiter verfügen in diesem Bereich über eine nur eingeschränkte Mitsprache und haben damit keine größeren Spielräume für eigenständige Entscheidungen im Bereich der Kosten und Wirtschaftlichkeit. „Die Aufgabe des Betriebsleiters im engeren Sinne beinhaltet, den ihm anvertrauten Betrieb im Rahmen der von Geschäfts- oder Zentralbereich gemachten Vorgaben selbständig zu führen“ ${ }^{* 86}$ In allen Personalfragen, personellen Angelegenheiten hingegen haben die Betriebsleiter eine sehr hohe Autonomie und verfügen über Einstellungen und Entlassungen von Beschäftigten. ${ }^{87}$ Außerdem liegt die gesamte Organisation, Disposition, Führung, Unterweisung und Qualifikation in den Händen der Leitung. Personaleinsatzkonzepte und Arbeitsorganisation werden nicht von den Personalabteilungen geplant und vorgelegt, sondern vom betrieblichen Managements individuell im Rahmen der gegebenen technischen Bedingungen umgesetzt. Die Aufgabenverteilung innerhalb der Betriebsleitung differiert insofern, als der Bereichsingenieur seinen Schwerpunkt eher auf Prozessoptimierung im Bereich Technik, der Betriebsleiter eher im Bereich der Produktion hat. Doch lässt sich diese Aufteilung nur schwer auf alle Chemiebetriebe beziehen. Die Konstellationen reichen hier von kleineren Betrieben, in denen die Funktionen der Betriebsleitung in Personalunion ausgeübt werden bis zu Betrieben, in denen es mehrere Betriebsleiter bzw. Ingenieure gibt. Insgesamt aber basiert die Betriebsführung Ende der 1980er Jahre paritätisch sowohl auf chemischen wie verfahrenstechnischen Kompetenzen. Hier kommen einmal die bereits erwähnten Reorganisationsmaßnahmen im Bereich der Instandhaltung zum Tragen. In dem Maß, in dem sich in den 1980er Jahren die Überzeugung durchsetzt, dass Betriebssicherheit, Erträge und Kostenreduktion nicht allein von stofflich-chemischen Prozessen und Innovationen abhängen, sondern auch mittels technischer Optimierungen beeinflusst werden können, wächst die Relevanz der Verfahrenstechnik. Der Erfahrungsaustausch sowie eine möglichst frühzeitige Kooperation zwischen chemischer und verfahrenstechnischer Seite komme der Prozessoptimierung zugute, die Gleichrangigkeit der Fachdisziplinen in der Betriebsleitung unterstreicht diese Entwicklung. ${ }^{88}$

Der Betriebsleitung unterstellt sind die für das operative Geschäft zuständigen Betriebsmeister. Sie bilden die Schnittstelle zu den nicht-akademisch qualifizierten Akteuren im Betrieb und haben neben dem Betriebsleiter eine wichtige Position im Betrieb inne. Ihre „Karriere“ beginnt in der Regel als Anlagenfahrer, oftmals sind sie am Aufbau der Anlage mit beteiligt und verfügen von daher über eine umfassende Prozesskompetenz. In Abwesenheit der Betriebsleitung übernimmt der Betriebsmeister in einer Art Assistenzfunktion die Aufgaben der Leitung. Im Tagesgeschäft selbst ist die Kontrolle der anwesenden Schicht Hauptaufgabe, d.h. die Personalverantwortung liegt beim Betriebsmeister, was in einigen Betrieben auch bedeutet, dass die Urlaubs- und Arbeitseinteilung bei den Betriebmeistern liegt. Bei Störungen ist der Betriebsmeister die betriebliche „Feuerwehr“ und trägt die Verantwortung für die initiierten Maßnahmen zur Problemlösung. Im Rah-

\footnotetext{
${ }^{86}$ Richtlinie „Betriebsleitung in der Bayer AG“, 1993.

${ }^{87}$ Dies ist nicht zu verwechseln mit der Bestimmung der Besatzungsgröße in der Anfahrphase, also zum Betriebsstart. Diese Entscheidung wird zwischen technischer Planung, überbetrieblichem und betrieblichem Management unter Berücksichtigung des Anlagendesigns festgelegt.

${ }^{88}$ Die Gesamtverantwortung, und dies ist wiederum der chemiespezifischen Gefahrenlage zuzuschreiben, beschränkt sich allerdings auf eine Person, die in Ausnahmesituationen das alleinige Entscheidungsrecht (bzw. auch die Pflicht dazu) hat.
} 
men von Prozessoptimierung greift das betriebliche Management auf seine fachlichen Kompetenzen zurück.

Das operative Geschäft wird von den Schichtmannschaften erledigt und kann bezüglich der Arbeitsorganisation als „Organisationsform strenger Arbeitsteilung“ (Mickler et al. 1975:267) charakterisiert werden, d.h. es kommt zu einer

„(...) Konzentration von Arbeitsfunktionen ähnlichen Inhalts und Qualifikationsniveaus zu Arbeitsplätzen mit dem Ziel, höhere Qualifikationsanforderungen auf möglichst wenige Personen zu begrenzen, der Mehrzahl der Beschäftigten aber niedrig qualifizierte Funktionen zuzuschreiben. Dies führt [...] zu einer strikten Trennung zwischen den Hauptfunktionen, so daß die Produktions-, Labor- und Instandhaltungstätigkeiten jeweils verschiedenen Personengruppen zugewiesen und bei ihnen dauerhaft befestigt werden.“ (ebd.)

Auf Schichtebene bildet diese Organisationsform die Grundlage für eine Karriereleiter, die prinzipiell für jeden unabhängig von Vor- und Ausbildung zu erklimmen ist. Innerhalb der Produktionsmannschaften verläuft sie vom Anlagenfahrer über den Messwart zum Kolonnenführer und endet innerhalb der Wechselschicht beim (stellvertretenden) Schichtführer. Betriebszugehörigkeit und damit verbundenes Erfahrungswissen spielen für diesen Aufstieg eine größere Rolle als die Facharbeiterqualifikation und obgleich die Branche sich von einer Un- und Angelerntenindustrie zu einem Zweig wandelt, der verstärkt auf die Potentiale der Facharbeiter angewiesen ist, ändert dies nichts an den konkreten Arbeitsstrukturen, Aufstiegswegen und der hieraus resultierenden Segmentierung. Noch immer basieren, wir zeigten dies, die Mehrzahl der Betriebe auf einem Innovationsmodell, dass die umfassenden theoretischen Kenntnisse der Ausbildung systematisch vom Anforderungsprofil ausschließt.

Auf der untersten Stufe der schichtinternen Hierarchie stehen die Anlagenfahrer. Sie sind in der Regel für die Regulierung einer einzigen Anlage des Gesamtsystems zuständig. Ihre Hauptaufgabe besteht darin, die ihnen zugewiesenen Anlagenbereiche vor Ort zu überwachen und Interventionen durchzuführen, die von der Zentralwarte aus nicht zu leisten sind. Neben dieser Kernaufgabe übertragen die meisten Betriebe aus Rationalisierungsgründen einige Zusatzfunktionen an die Anlagenfahrer, etwa Laborhilfstätigkeiten, kleinere Instandsetzungen sowie in geringem Umfang Wartungsarbeiten. Für die Anlagenfahrer bedeutet dies, dass sie zwar eine relativ hohe Verantwortung gegenüber ihrer Umwelt tragen, aber nur einen kleinen Entscheidungsspielraum haben. Ihr Einfluss auf das Produkt beschränkt sich auf einen Ausschnitt, da sie vor Ort agieren und ihnen das Panorama der Messwarte fehlt. Der Überblick über den Gesamtprozess und damit verbundene Ausbringung und Qualität bleibt dem Betriebsleiter sowie den Meistern vorbehalten. Und auch in Störfällen ist der Anlagenfahrer nicht befugt, ${ }^{89}$ die nötigen Schritte einzuleiten, sondern ist auf den Vorgesetzten angewiesen, der die letztgültige Entscheidung trifft.

\footnotetext{
${ }^{89}$ An dieser Stelle sei darauf hingewiesen, dass eine fehlende Befugnis nicht gleichzusetzen ist mit einer fehlenden Qualifikation. Selbst wenn der Betroffene sich in der Lage sähe, die Situation eigenständig zu meistern, setzen die bereits erwähnten Sicherheitsvorschriften enge Grenzen.
} 
Geringfügig verändert sich diese Situation für den Messwart, die erste „Aufstiegspositi-

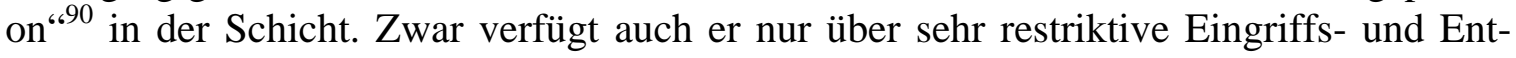
scheidungsbefugnisse, hat aber bedingt durch seine zentrale Position in der Messwarte eine größere Übersicht über das Geschehen. Seine Arbeit unterscheidet sich von der des Anlagenfahrers vor allem durch das starke Gewicht, dass Kontrollfunktionen gegenüber konkreten Eingriffen an den Produktionsanlagen bzw. in den Prozessablauf haben sowie der räumlichen Trennung von Arbeitsbereich und arbeitsrelevanter Umwelt.

Vorarbeiter, Kolonnen- und Schichtführer bilden die hierarchische Spitze, bleiben aber ein konstitutiver Bestandteil der Schicht, d.h., sie führen dieselben Arbeiten wie ihre Kollegen aus. Darüber hinaus sind sie die für die Schicht verantwortlichen Ansprechpartner für Meister, Betriebsleitung und indirekte Bereiche. Im Normallauf ebenso wie im Störungsfall sind sie weisungsbefugt, sofern kein direkter Vorgesetzter im Betrieb ist. Sie teilen, so dies nicht vom Betriebsmeister übernommen wurde, die Arbeit ein und verwalten die Urlaubspläne. Die Aufstiege innerhalb dieser Struktur auf dem Shop floor sind abhängig von den praktisch-empirischen Qualifikationen der Beschäftigten, die sie sich im Lauf ihrer Betriebszugehörigkeit erworben haben.

\subsubsection{Kooperations- und Statusmodell}

Diese formale Struktur wird ergänzt durch aus der chemiespezifischen Situation resultierende Faktoren: ${ }^{91}$ Erstens ist eine hohe räumliche Nähe der Akteure zueinander zu konstatieren, so dass die bereits erwähnte strikte hierarchische und funktionale Trennung sich in der betrieblichen Realität kaum wahrnehmen lässt. Die Messwarte ist Dreh- und Angelpunkt des Geschehens, Schicht- und Betriebsmeister gehen dort ebenso selbstverständlich ein und aus wie Ingenieure, Laboranten, Handwerker etc. Durch diese „Alle(s) unter einem Dach“ Atmosphäre gibt es nicht die „anonymen Anderen“, sondern kennen sich meist alle am Produktionsprozess Beteiligten beim Namen. Der Arbeit in Chemiebetrieben ist immer auch Gefährdungspotential inhärent, und so prägt zweitens das Wissen um das gemeinsam getragene Risiko und das daraus resultierende Verantwortungsbewusstsein in besonderer Weise die Sozialbeziehungen. Wo Fehler in den Produktionsbereichen in ihren Auswirkungen eher mittelbar die Konsumenten treffen, wirken Fehler in der Chemieproduktion im Gegensatz dazu unmittelbar und mit einer potentiell hohen Breitenwirkung. Das so entstehende Risikobewusstsein (welches seine konkrete Umsetzung im Arbeitsprozess in unzähligen Dokumentations und Berichtspflichten findet) führt in seiner negativen Definition zu einer starken Kontrollmentalität, die sich aber positiv gewendet auch in einem hohen Vertrauen niederschlägt, wenn beispielweise die Nacht- und Wochenendschichten ohne einen Vertreter der Betriebsleitung arbeiten. ${ }^{92}$ Im Gegensatz zu anderen Branchen zeichnet sich die Arbeit in der chemischen Produktion noch immer

\footnotetext{
${ }^{90}$ Der Begriff steht in Anführungszeichen, da für viele Beschäftigte die Arbeit in der Messwarte wegen ihrer räumlichen Isoliertheit nicht unbedingt attraktiv erscheint und sie deswegen lieber als Anlagenfahrer arbeiten. Dennoch ist es eine betrieblich höher eingestufte Position.

${ }^{91}$ Die einzelnen Dimensionen gehen zurück auf Kotthoff, Reindl (1990), die diese zur Analyse der betrieblichen Sozialordnung verwenden.

${ }^{92}$ Dieses schlägt sich auch in den unternehmensinternen Stellenbeschreibungen nieder, und bei der Bayer AG etwa zählt „Verantwortungsbewusstsein“ vom Chemikanten bis zum Betriebsleiter zum Anforderungsprofil, und auch im unternehmenseigenen Werbeslogan „Kompetenz und Verantwortung“ kommt die Gleichrangigkeit von Fähigkeiten und Bewusstsein zum Ausdruck.
} 
durch ein hohes Maß an Erfahrungswissen aus. Unabhängig vom Qualifikationsniveau heißt dies: Wer neu im Betrieb ist, muss die Tücken des Prozesses erst am eigenen Leib erfahren, ein Gespür für das Regelwerk bekommen und kann sich nicht auf Zahlen und Schaubilder verlassen. So machen drittens alle Akteure in ihrer betrieblichen Sozialisation eine recht ähnliche Grunderfahrung. Sie umfasst nicht nur die informellen sozialen Regeln und Normen, die sich jeder - egal in welcher Organisation- aneignen muss, der in eine Gruppe neu hinzukommt. Vielmehr geht es um das Wissen um die Produktion, welches die 'alten Hasen' von den 'Frischlingen' trennt. So kann es durchaus sein, dass der ungelernte Anlagenfahrer dem diplomierten Ingenieur in seinem Bereich zeigt, „wo es langgeht“. Aus diesem Grund ist die Fluktuation in den Betrieben äußerste gering: Ist der aufwendige Prozess des Anlernens erst einmal abgeschlossen, bleiben die Beschäftigten meist über Jahre hinweg in einer Schicht zusammen, und diese personelle Stabilität und Kontinuität ist ein wichtiger Faktor für die Herausbildung eines gemeinschaftlichen Verbundes.

Diesen eher auf einen engen Sozial- und Kommunikationszusammenhang verweisenden Faktoren steht das vom Primat der Verwissenschaftlichung geprägte Kooperations- und Statusmodell entgegen. Die Innovationsfunktionen sind vertikal einseitig produktionsfern verortet, und so dominieren Kooperationsformen, die von recht eindeutigen Über- und Unterordnungsverhältnissen strukturiert werden.

Für die Beschäftigten setzt einmal die technische Modernisierung den Rahmen für Kooperationsmöglichkeiten, d.h. es gibt Formen der Zusammenarbeit, die der Arbeit inhärent sind, und die sich aus der „sozialen Bedingtheit der Arbeit durch die Arbeit selbst“ (Popitz et al. 1957) ergeben, ohne dabei deren technische Dimension außer acht zu lassen. Das technisch bedingte Mindestmaß an Zusammenarbeit der Beschäftigten in der Chemischen Industrie wird in früheren Untersuchungen als teamartig (Popitz et al. 1957) bzw. technisch - kolonnenartig (Kern, Schumann 1985) bezeichnet und verweist auf eher positive Bedingungen für Kooperation. Technisch bedingt sind die einzelnen Arbeiter nicht an einen festen Arbeitsplatz, wie etwa am Fließband, gebunden, sondern sie können sich frei bewegen. Die Verteilung der Arbeitsaufgaben auf die einzelnen Arbeitskräfte könnte somit in unterschiedlicher Weise erfolgen und von den Arbeitern disponiert werden. Der einzelne kann darüber hinaus die ihm übertragene Arbeit, in gewissen Grenzen, einteilen, da durch die technische Anlage keine „Taktung“ vorgegeben, sondern ein größerer zeitlicher Rahmen gegeben ist. Teamartig kooperierende Arbeitskräfte sind daher in der Lage, sich gegenseitig zu unterstützen; jeder kann sich unmittelbar an der Arbeit eines anderen beteiligen und in Stresssituationen dessen Arbeit mit übernehmen (Popitz et al. 1957). Kern und Schumann (1985) definieren spezifische Elemente dieser Zusammenarbeit knapper mit

„1. gegenseitige Unterrichtung und Beratung; 2. wechselseitige aktive Unterstützung in Notsituationen; 3. Interdependenz zwischen denjenigen Arbeitern, die unterschiedliche Teile derselben Anlage betreuen.“ (ebd. 145)

Dass diese recht positive Bestimmung vor allem im Hinblick auf die genannten dispositiven Aufgaben wie die Arbeitseinteilung einiger Einschränkungen bedarf, liegt, rufen wir uns die schichtinterne Hierarchie sowie den arbeitsorganisatorischen Konservatismus in Erinnerung, auf der Hand. Wir haben bereits zeigen könne, dass es Gestaltungsvarianten 
gibt, in denen die Eingriffsbefugnisse und Entscheidungskorridore der Beschäftigten durchaus hoch sind. Zwar setzt die Technologie für die Organisation von Arbeit Grenzen und limitiert Gestaltungsmöglichkeiten, sie determiniert diese aber nicht (Hirschhorn 1984:4). Im traditionell-hierarchischen Industriebetrieb, der Ende der 1980er Jahre charakteristisch für die Chemische Industrie ist, bleibt die Kooperation der Beschäftigten allerdings auf ein Minimum beschränkt.

Im Normallauf der Anlage, d.h. bei störungsfreiem Ablauf der Prozesse, ist die Kooperation zwischen den Beschäftigten äußerst gering. Die Anlagenfahrer arbeiten räumlich weitgehend getrennt voneinander an den unterschiedlichen Anlagenteilen, nur ab und zu kooperieren Messwart und Anlagenfahrer und tauschen routinemäßig Informationen über bestimmte Prozessabschnitte aus. Kommunikation zwischen den Beschäftigten auf Schicht findet jenseits der technisch bedingten statt, vor allem in der Messwarte, die in den meisten Chemieunternehmen zentraler Treffpunkt ist.

Erst beim Auftreten größerer Störungen verändert sich die Kooperationsdichte, da eine Irritation des prozesshaften Ablaufs der Produktion meist auch Interventionen in vor- und nachgelagerten Anlagenteilen nach sich zieht. Ein schnelles, kollektives Eingreifen aller Anlagenfahrer ist deshalb nötig, um ein Übergreifen der Störung auf den gesamten Prozess zu verhindern.

„Unter diesen Umständen gilt die gegenseitige Hilfeleistung der Anlagenkontrolleure als eine ganz selbstverständliche Aktualisierung des sonst mehr als latenten Kooperationsbezugs, zumal jeder einmal in eine ähnliche Notlage kommen kann, er aber vermittelt über das interdependente Anlagensystem auch selbst betroffen ist.“ (Mickler et al. 1975:425)

Die Koordination erfolgt, je nach Brisanz der Ausnahmesituation, von der Messwarte aus durch Meister und Betriebsleitung. Die Anlagenfahrer erörtern dann gemeinsam mit dem Wartenfahrer und dem Meister komplizierte Interventionen, d.h. Eingriffe, die komplizierte Verfahrensfragen betreffen und eine genaue Planung und Abstimmung der Aktion unter Berücksichtigung des gesamten Produktionsgeschehens erfordern. Dies darf jedoch nicht darüber hinwegtäuschen, dass auch diese Form der Kooperation mit Vorgesetzten einer recht eindeutigen top-down Linie im Anweisungsstil erfolgt. So sind die Anlässe zur Kommunikation recht häufig, doch verbindet sich damit kein echter fachlicher Austausch oder ein gleichberechtigter Umgang. Am deutlichsten wird dies daran, dass Terminvorgaben, Produktwechsel oder Maschinenstillstände in der Regel kurzfristig und unabgesprochen mit den Produktionsarbeitern geschehen. Da die Beschäftigten keine Informationen oder Begründungen für die eingeleiteten Maßnahmen bekommen, ist ihnen ein Nachvollziehen unmöglich und sie bleiben zurückgeworfen auf routinemäßiges Ausführen. Es erstaunt wenig, dass eine Beteiligung der Beschäftigten am Innovationsprozess in diesem Modell systematisch nicht vorgesehen ist. Kern und Schumann verweisen in ihrer Studie „Industriearbeit und Arbeiterbewusstsein“ (1970), darauf, dass Produktionsarbeiter dennoch „Verbesserungen“ vorantreiben, allerdings nicht aus einem betrieblich induzierten Rationalisierungskalkül heraus, sondern zur Reduktion individueller Belastungssituationen und häufig informell. Zwar steht in der Mehrzahl der Chemieunternehmen ein betriebliches Vorschlagswesen zur Verfügung, doch erstens ist es nicht immer im Sinn der Beschäftigten, ihre Tricks und Kniffe offen zu legen. Hier spielt technisch bedingt viel weniger das Problem der Beteiligung an Rationalisierung und eventuellem Personalabbau eine Rolle. Gerade in der Chemischen Industrie ist es die hohe Regelungsdichte, die die Beschäftigten dazu zwingt, ihre „persönliche Note“ nicht offen zu legen, da es sich nicht 
selten um durch die geltenden Vorschriften nicht abgedeckte Handlungen handelt. Zweitens schließlich zeigen auch die Vorgesetzten oftmals nur ein geringes Interesse an den Vorschlägen der Beschäftigten. Die „Produktionsintelligenz“ steckt ihrer Ansicht nach nicht in den Köpfen der Arbeitenden, sondern liegt bei den Innovationsspezialisten aus Instandhaltung, Ingenieurwesen oder naturwissenschaftlicher Fachrichtung. Verstärkt wird diese statusrelevante Trennung zwischen Anweisenden und Ausführenden gemäß der Formalstruktur durch den Faktor Zeit. Da die Produktionsmannschaften im Schichtrhythmus arbeiten, treffen sie nur unregelmäßig mit der Tagschicht zusammen. Die Beschäftigten sind an ihre Schichtzeiten gebunden, für das betriebliche Management einschließlich der Instandhaltung gilt der „Normalarbeitstag. Die hierarchische und funktionale Separation von Tag- und Wechselschicht tritt deutlich zutage und wird nach außen hin noch durch eine traditionelle „blue collar - white collar“ Grenze gestützt. ${ }^{93}$

Ganz ähnlich verhält es sich auch für die Zusammenarbeit mit dem Labor. Die Betriebslabore, die nicht nur die Produktqualität kontrollieren, sondern auch in gewissem Maß Forschungsarbeit in Kooperation mit dem betrieblichen Management und dem Zentrallabor leisten, gehören zur Grundausstattung der Produktionsstätten. Das Betriebslabor bildet zwar die Schnittstelle zwischen Forschung und Produktion, die Arbeit im Labor hat jedoch nichts mit der Arbeit der Chemikanten zu tun. Aus den großen Mengen an Produkt erhalten die Laboranten kleine Proben aus dem laufenden Prozess, die nach wissenschaftlichen Methoden überprüft werden. Die Kooperationsbeziehungen sind also auf ein äußerst geringes Maß reduziert, im wesentlichen werden die Werte der Routineanalysen an die Messwarte durchgegeben und der direkte Kontakt beschränkt sich auf Probenübergaben. Und auch informelle Kontakte sind wegen der räumlichen als auch zeitlichen Distanz zwischen Labor und Anlage eher selten. Obwohl das Labor räumlich im Betrieb angelagert ist, wie die Anlagen, ist der Kontakt zu den Schichten auf die jeweiligen Probenzieher beschränkt, Kommunikation und Kooperation sind eher marginal. Eine deutliche symbolische Grenze zwischen Labor und Produktion bildet die Arbeitskleidung: Im Labor wird traditionell in den weißen Kitteln gearbeitet, die auch das betriebliche Management trägt und dem sich das Labor eher zugehörig fühlt, Produktionsarbeiter tragen blaue Arbeitskleidung.

Der Kontakt zwischen Instandhaltungshandwerkern und Produktionsarbeitern beschränkt sich vor allem auf die Zusammenarbeit im Störungsfall. Der Instandsetzer nimmt dabei zunächst Kontakt mit der Messwarte auf und erhält so erste Informationen über Grad und Ausmaß der Störung. Der für das beschädigte Anlagenteil zuständige Anlagenfahrer unterstützt den Instandsetzer dann vor Ort bei der Fehlersuche. Entsprechend der hauptsächlichen Einsatzfelder der unterschiedlichen Instandhaltungsgruppen ist der Kontakt zwischen Schlossern und Anlagenfahrern eher enger, während die Elektriker und Prozessleitelektroniker hauptsächlich zu den Messwarten in Beziehung stehen. Von einer echten Zusammenarbeit zwischen Instandhaltung und Produktion kann aufgrund der hohen Regelungsdichte in der Chemischen Industrie nicht die Rede sein. Deutlich wird dies bereits daran, dass eine Schadensmeldung im Normalfall von den Produktionsarbeitern nicht direkt an die Instandhaltung geht, sondern ihren Weg über die hierarchischen Stufen der Produktion zum (Betriebs-) Meister hinauf nimmt, dieser den Instandhaltungsmeister ver-

\footnotetext{
${ }^{93}$ Der weiße Kittel steht in der chemischen Industrie eindeutig für die wissenschaftliche Ausbildung, während der blaue Kittel (vom Anlagenfahrer bis zum Betriebsmeister) den „Praktiker“ markiert.
} 
ständigt und dann die hierarchische Treppe zurück zu den Instandhaltungshandwerkern nimmt.

Verschärft wird die Demarkationslinie zwischen Produktionsbeschäftigten und Instandhaltungshandwerkern auch durch den Sitz der IH, die in den meisten Unternehmen in Zentralwerkstätten organisiert ist. Obgleich in den 1980er Jahren im Zuge umfassender Reorganisationsmaßnahmen die Instandhaltung näher an die Produktion geführt wurde und es teilweise operative Zuständigkeiten einzelner Schlosser oder Prozessleittechnik (PLT) - Handwerker für bestimmte Betriebe gibt, so bleibt die „Heimat“ der Handwerker aber „ihre“ Werkstatt: Dort sitzt ihr Meister, treffen sie ihre Kollegen und haben sie ihre Pausenräume. So kann zwar eine deutlich höhere Vor-Ort-Präsenz der Instandhalter konstatiert werden, ein Erfahrungsaustausch zwischen IH und Produktion als gleichberechtigte Kommunikation und Kooperation bleibt aber aus.

Diese auf den ersten Blick fachliche Begründung für die Schnittstelle zwischen Produktion und IH wird verstärkt durch das deutsche Modell der Berufsfachlichkeit und der damit verbundenen Identifikation der Facharbeiter mit ihrem Beruf. Auch und gerade im eigenen Selbstverständnis der IH genießt fachliches Können hohen Stellenwert, was wiederum zu Abschottung gegenüber den Produktionsmannschaften führt. Innerhalb der Chemiebelegschaften haben die Instandhalter eine klare Sonderrolle inne, da sie einen ,richtigen' Facharbeiterberuf gelernt haben und diesen im Gegensatz zu den Chemikanten tatsächlich ausüben. Als ,praktizierende’ Facharbeiter belegen sie im betrieblichen Statusgefüge einen Platz, der dem Anlagenfahrer überlegen ist. Ihre Position erreichen sie nicht erst auf dem Weg des Aufstiegs, sondern sie steigen als Facharbeiter mit einem selbstverständlichen, individual- und kollektivrechtlich gesicherten Anspruch in die Betriebe ein, für den sie nicht erst betriebliches Erfahrungswissen vorweisen müssen. Darüber hinaus erfolgt die Kompetenzverteilung zwischen Arbeitern und Vorgesetzten nicht dem Muster der direkten Produktion, also ,oben fast alles, unten fast nichts'. Die Instandhaltungsarbeiter besetzen vielmehr Kompetenzclaims, in die ihre Meister als nicht mehr praktizierende Handwerker weder richtig hineinschauen und hineinregieren können (Kern, Schumann 1990:282).

Und auch zwischen den Arbeitskrafttypen der einzelnen Gewerke, also traditionelle Instandhaltungsberufe auf der einen und modernere Instandhaltungsspezialisten auf der anderen Seite kann noch einmal differenziert werden in Schlosser und Elektriker gegenüber Mess- und Regelmechaniker bzw. Prozessleitelektroniker. Die „alten“ InstandhaltungsHandwerker definieren ihre Arbeit nicht allein über berufliches Können, sondern körperlicher Einsatz sowie die Einbindung in Kollektivvollzüge gehören genauso zum Leistungsverständnis dazu. Damit entspricht ihr Arbeitstyp einem beruflich traditionellen, d.h. Leistung wird als Kollektivleistung definiert und beruht auf beruflichem Können und physischem Einsatz. Dem steht auf der anderen Seite der individuell-technokratisch geprägte Instandhaltungsspezialist gegenüber. In diesen neuen und vor allem von jüngeren Beschäftigten besetzten Berufen ist die traditionelle Handwerkstätigkeit passé.

„Dazu fehlt es zu sehr an den Merkmalen der Körperlichkeit und Kollektivität; einen Hammer nimmt ein Meß- und Regelmechaniker nicht mehr in die Hand, und seine Kontakte zu 
Berufskollegen bewegen sich mehr auf der Ebene des Persönlichen. [...] der Meß- und Regelmechaniker ist ein hochkompetenter Einzelarbeiter.“(Kern, Schumann 1990:283) ${ }^{94}$

Das betriebliche Sozialgefüge ist trotz der deutlichen formellen Segmentierung geprägt durch „eine einigermaßen komplizierte Mischung aus kollegialen und hierarchischen Momenten“, die sowohl auf Schichtebene als auch zwischen Produktionsmannschaften, Instandhaltung und betrieblichem Management existiert (Kern, Schumann 1990:258). Die besonderen Produktionsbedingungen forcieren und beschränken teamförmiges und fachübergreifendes Arbeiten gleichermaßen. Die Einbindung der Betriebs in das Unternehmen erfolgt hauptsächlich über die Betriebsleitung, für die Beschäftigten bleibt ihr Betrieb die „Monade“ und der Kontakt zu anderen Betrieben ebenso wie zu Labor und Instandhaltung ist auf ein Minimum beschränkt. Aus der diesem Modell zugrunde liegenden Polarisierung von Innovations- und Produktionsfunktion resultiert eine unterschiedliche Verteilung von Privilegien. Während auf der einen Seite Instandhaltung und betriebliches Management Möglichkeiten zur beruflichen Entfaltung, zu kreativem Handeln haben und innerhalb ihres Aufgabenbereiches mittels hierarchischer Legitimation über ein hohes $\mathrm{Ma}$ an Autonomie verfügt, gilt für das Gros der Beschäftigten in der Produktion das Gegenteil, sie bleiben die „Lückenbüßer der Mechanisierung“. Bei aller räumlichen Nähe dominiert die recht starre Abschottung der Funktionsbereiche, und ein deutlicher Zusammenhang ist auszumachen zwischen der exklusiven Definition und Zuordnung von Innovationskompetenz und Statusdifferenzierung (Baethge, BaethgeKinsky 1998).

\subsection{Zwischenresümee}

Bei aller Heterogenität der Branche kann von einem dominanten unternehmerischen Modell zur Verbindung zweier sehr unterschiedlicher Kompetenzbereiche ausgegangen werden. Zum einen handelt es sich dabei um die Innovationskompetenz der Unternehmen, die sich über „langfristig aufgebauten, auf hochspezialisierte Arbeitskräfte in anspruchsvollen Kooperations- und Erfahrungszusammenhängen gegründeten Forschung, die extrem teuer ist und mit hohem Risiko für vielfach noch nicht existierende Märkte produziert“ (Kädtler 1999:25) definiert. Diesem risikoreichen Geschäft steht zum anderen eine komplexe Industrieproduktion gegenüber, die „auf der Grundlage hoher Kapitalintensität, kontinuierlicher Ausschöpfung technischer Entwicklungs- und inkrementeller, empirischer Verbesserungspotentiale für ein breites Spektrum von Märkten produziert“ (ebd.). Beide Funktionen sind eng miteinander verbunden und finden ihren organisatorischen Ausdruck in der integrierten Zentralforschung, der Verbundchemie sowie bezogen auf die Arbeitskräfte in Versuchen, über eine übertarifliche Entlohnung und umfassende Sozialleistungen eine langfristige Bindewirkung an den im Vergleich zur Ausbildung eher unattraktiven Beruf in der Produktion zu erzielen.

Historisch ist diese Entwicklung über eine sowohl im akademischen Bereich als auch auf der Ebene der Instandhaltung stattfindende Ausdifferenzierung der Personalstruktur begründet (Abb.3). Die science based industry entwickelt sich auf der Basis von Qualifikationsstrukturen, die durch einen hohen Anteil von Angelernten geprägt sind, denen eine relativ kleine Gruppe von „professionellen“ Chemikern gegenübersteht. Grundlage und

\footnotetext{
${ }^{94}$ Dies gilt ebenso für den seit 1986 existierenden Nachfolgeberuf des Prozessleitelektronikers.
} 
Voraussetzung der Ausdifferenzierung der Professionen in der Chemischen Industrie ist die Kapitalisierung von Wissen. Sie findet ihren Ausdruck in den beschriebenen institutionellen Verbindungen zwischen Akademie und Industrie. Die Industrie macht sich die klassischen Funktionen der Universitäten, Forschung und Lehre zu nutze, indem sie sich die daraus resultierenden Leistungen der Universitäten zur Organisation ihrer Innovationsprozesse aneignet. Die wissenschaftlichen Grundlagen für neue Verfahren und Produkte, also neues, an den Universitäten produziertes Wissen, werden internalisiert. Zugleich wird auf das an den Universitäten ausgebildete Personal, sowie deren wissenschaftlichen, sozialen und methodischen Kompetenzen zurückgegriffen. Über diesen Prozess der Verklammerung gelingt die Umwandlung von Wissen in eine neue, für wirtschaftliche Zwecke geeignete Produktivkraft (Etzkowitz, Webster 1998).

Insgesamt basiert das Produktionskonzept der Chemischen Industrie auf diesem wissenschaftlichem Wissen und hoher handwerklicher Kompetenz. Die Arbeit in der direkten Produktion ist weitgehend von Innovationsprozessen abgekoppelt, und mit zunehmender Technisierung verändert sich die Personalstruktur dahin gehend, dass es zu einem deutlichen Überhang an „professionellen“ Professionen kommt. Ende der 1980er Jahre ist bedingt durch eine stetige funktionale Ausdifferenzierung auf der wissenschaftlichinstandhalterischen und eine Personalreduktion auf der Produktionsseite das Verhältnis von Arbeitern zu Akademikern beinah gleichgewichtig. Auf der Ebene der Produktion ist die Chemische Industrie damit schon immer eine Facharbeiterbranche, „nur eben keine der Produktionsfacharbeiter“ (Schumann et al. 1994:574). Zwar steigt auch in den 1980er Jahren der Anteil an Chemikanten signifikant. Ursächlich sind einmal die stetig steigenden Umwelt- und Sicherheitsauflagen. Sie ziehen Anforderungen nach sich, die sich etwa in dem erheblichen Dokumentationsaufwand der „Good Manufacturing Practices (GMP)“95 niederschlagen und in vielen Bereichen der Chemischen Industrie den Facharbeitereinsatz vorschreiben. Außerdem ist der Prozess der Technisierung insgesamt gekennzeichnet durch eine fortschreitende Reduktion des Bedienpersonals, der im Zeitverlauf die Grenzen dieser Rationalisierung aufgezeigt. Der Schritt zur zentralen Messwarte hat die Arbeit an chemischen Anlagen grundlegend verändert und zu einem erheblichen Personalabbau geführt, so dass das Potential für weitere Personaleinsparungen „weitestgehend ausgeschöpft“ erscheint (Tacke 1997:155). Die Betriebsleitungen müssen daher qualifizierteres Personal einsetzen, um die Sicherheit des Produktionsprozesses auch bei „Unterbesetzung“ zu gewährleisten.

\footnotetext{
${ }^{95}$ Vorschriften, die deshalb von den Beschäftigten in den Betrieben auch mit „Give Me Paper“ oder „Große Mengen Papier“ übersetzt werden.
} 


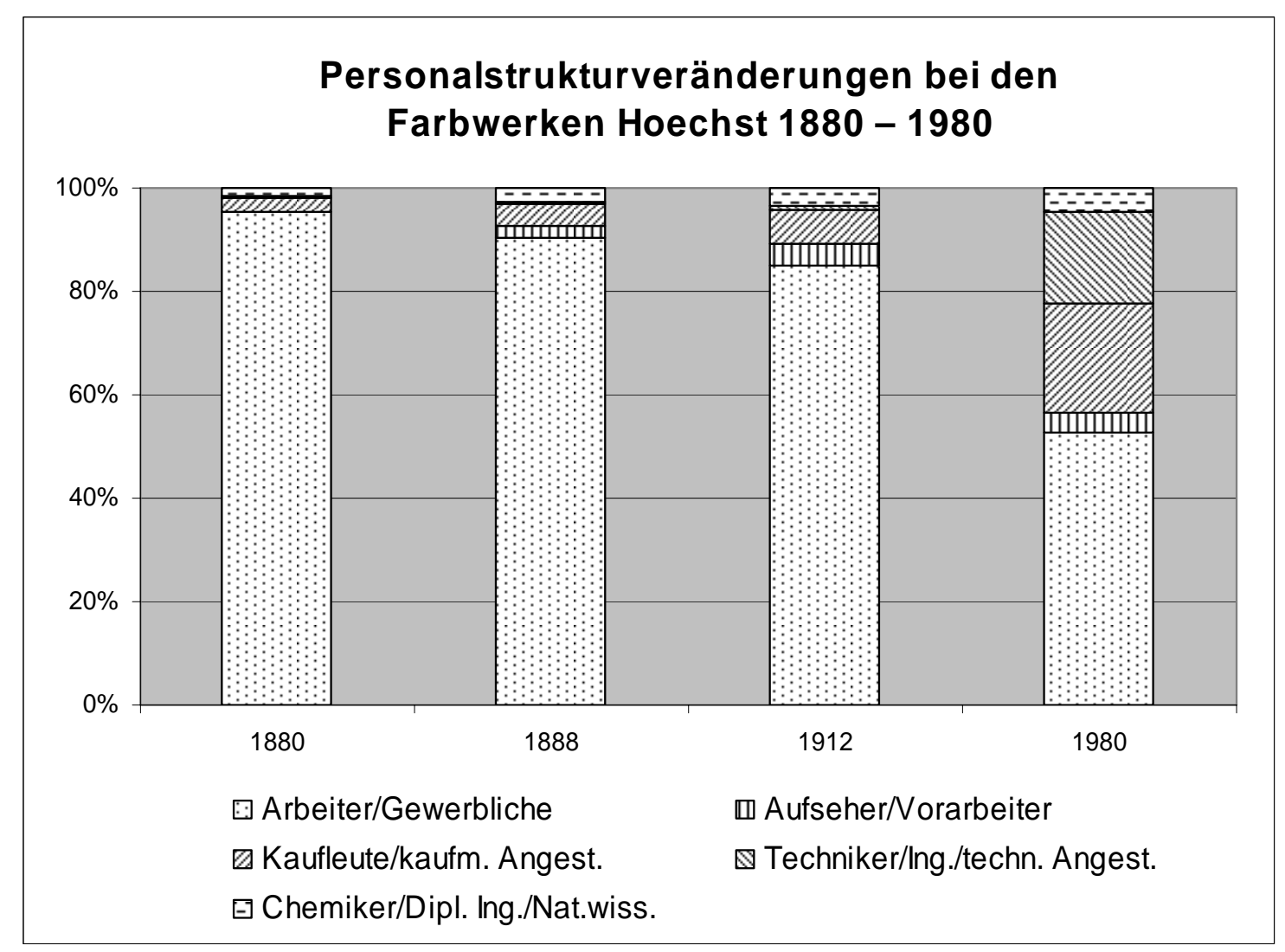

Abbildung 4: Personalstrukturveränderungen bei den Farbwerken Hoechst; Hack, Hack (1985)

So kann die Chemische Industrie Ende der 1980er Jahre unter formalen Bedingungen als Facharbeiterbranche bezeichnet werden, zu einem flächendecken wirksamen Wandel des Anforderungsprofils ist es in der Chemischen Industrie jedoch nicht gekommen. Dies zeigt sich an der Trennung zwischen produktionsfern angelagerten Innovationsfunktionen und den auf den Shop floor konzentrierten Gewährleistungsfunktionen. Innovationsanforderungen, d.h. Planung, Steuerung und Optimierung des Arbeitsprozesses werden an die Chemikanten seitens des Managements nicht gestellt, und das betriebliche Statusmodell verschärft diese Demarkationslinie noch. Für die Beschäftigten heißt dies, dass ihnen die Grundlage für Innovationshandeln, d.h. die kommunikative und kooperative Vernetzung mit anderen Akteuren, fehlt.

Diese mangelnde Wertschätzung, die der Produktionsarbeit in der Chemischen Industrie beigemessen wird, resultiert dabei in erster Linie aus ihrem Status als wissenschaftsbasierter Industrie, der die akademisch-technischen Borniertheiten der „professionals“ gegenüber den Arbeitskräften legitimiert und konserviert. Bernoux (1997) stellt im Hinblick auf die ,qualifizierten Berufsarbeiter' in deutschen Unternehmen fest, dass diese eine wichtige Rolle spielen, weil sie üblicherweise viele Beziehungen zu anderen Akteuren haben, man auf sie höre und ihrer Auffassung Rechnung trage (ebd. 281). In der Chemischen Industrie hat dies seine Gültigkeit einzig für die ,Berufsarbeiter’ der produktionsfernen Instandhaltungsbereiche. Der Produktionsarbeiter in der Chemischen Industrie bleibt, ob Facharbeiter oder angelernt, aus dem Innovationsapparat ausgeschlossen. 
Die Polarisierung der Anforderungen in jenseits des Shop floor angesiedelte Innovationsfunktionen und Gewährleistungsaufgaben für die Produktionsbeschäftigten verfestigt ein Status- und Hierarchiemodell, das erhebliche Folgen für die Arbeitssituation hat und zu einer Ungleichverteilung günstiger Arbeitsbedingungen führt. Möglichkeiten zur beruflichen Identifikation und den damit verbundenen inhaltlich anspruchsvollen, kreativeren Tätigkeiten bestehen insbesondere im Bereich der Forschung und Entwicklung, die jedoch, wir konnten dies anhand der Tätigkeitsprofile der Betriebsleitung zeigen, bis in die Produktionsbetriebe hineinreicht und die in der Chemischen Industrie durch die im „white collar" gekleideten Akademiker symbolisiert wird. Ähnlich stellt sich die Situation für die auf handwerklicher Tradition basierenden Tätigkeiten im Rahmen der Instandhaltung. Da sich die Unternehmen der Chemischen Industrie aus Kostengründen mehr und mehr auf das Outsourcing planbarer Instandhaltung fokussiert, zeichnen sich die verbleibenden Funktionen durch ein hohes Maß an Fachlichkeit aus. Für die Instandhaltungsspezialisten sichert dies nicht nur inhaltlich anspruchsvolle Arbeit, sondern darüber hinaus eine hohe Anerkennung dieser Arbeit im Vergleich zu den Beschäftigten in der Produktion. Unberücksichtigt bleiben in punkto Technikgestaltung und Arbeitsorganisation hingegen die Bedingungen zur Entfaltung und Weiterentwicklung der Produktionsintelligenz auf dem Shop floor. In der Chemischen Industrie hat dies zur Folge, dass in den Unternehmen seit den 1970er Jahren in der Summe widersprüchliche Strategien verfolgt werden. Die Innovationsspezialisten treiben mittels technisch-wissenschaftlicher Prinzipien Prozessinnovationen voran und schränken die Entfaltung von Produktionsintelligenz deutlich ein. Hauptsächlich aus sicherheitspolitischen Gründen wird parallel dazu die Aufqualifizierung der Beschäftigten zu Chemiefacharbeitern verstärkt. Dass die damit sich verschärfende Diskrepanz zwischen Anforderungs- und Kompetenzprofilen der Beschäftigten keine allzu negativen Auswirkungen auf die Motivation der Arbeitskräfte hat, liegt einmal darin begründet, dass die Chemieunternehmen bereits vor dem Zweiten Weltkrieg, wesentlich offensiver nach 1945, eine arbeitskraftpflegende Personalpolitik betreiben. ${ }^{96}$ Überdurchschnittliche Löhne, umfassende Sozialleistungen und eine hohe Arbeitsplatzsicherheit gelten bis Ende der 1990er Jahre als wirksame Prophylaxe gegen eine hohe Fluktuation und machen die von Schichtarbeit geprägten Arbeitsbedingungen im Branchenvergleich relativ attraktiv. Resümierend kann festgehalten werden, dass den Unternehmen an einer Sicherung der Produktionsintelligenz gelegen ist, diese aber im traditionellen Rationalisierungskalkül auf ein sehr eng gefasstes Verständnis eines speziellen betrieblichen, anlagenbezogenen Erfahrungswissen der Beschäftigten reduziert wird. Hier wirkt auch ein aus der Verwissenschaftlichung der Produktion resultierendes Superioritätsempfinden des Managements nach: Das Wissen der Arbeiter um den Produktionsprozess wird als unterlegenes, da nicht wissenschaftlich fundiertes betrachtet. Dass dieses Wissen und die damit verbundenen Kompetenzen durch ein Aufweichen der strengen Arbeitsteilung freigesetzt und für betriebliche Innovationsprozesse genutzt werden könnte, zählt gerade den Vorgesetzten in stark verwissenschaftlichten Prozessen kaum etwas.

\footnotetext{
${ }^{96}$ Historisch versuchten die ersten Chemiefabriken durch diese Anreize, überhaupt an Arbeitskräfte zu kommen. Später wandelte sich die Motivlage, man wollte vermeiden, dass gute Arbeitskräfte Betriebsgeheimnisse an die Konkurrenz weitergeben. Der Stellenwert des Erfahrungswissens spielte indes, wir zeigten dies, bald keine Rolle mehr. Dennoch waren die Unternehmen auf loyale Beschäftigte angewiesen. Die Technisierung hatte die Prozesse einmal immer störungsempfindlicher gemacht, so dass sie eines verantwortungsvollen Umgangs bedurften. Darüber hinaus hatten sich aber die Investitionen in Anlernzeiten drastisch erhöht, und interessierte man sich aus der Innovationsperspektive wenig für die Produktionsintelligenz, so konnte man in der Alltagspraxis nicht auf sie verzichten.
} 
Diese etablierte Organisation der Arbeitsteilung beinhaltet für die Unternehmen einige Vorteile. Die Begrenzung der Summe aller zu verrichtenden qualifizierten Tätigkeiten auf einige wenige Personen und Arbeitsplätze (Messwarte) senkt den Qualifizierungs- und Schulungsbedarf sowie die Personalkosten für Fachkräfte. Darüber hinaus machen die strengen Sicherheitsauflagen und gesetzlichen Vorschriften eine Nachvollziehbarkeit einzelner Verfahrensschritte notwendig. Je weniger Aufgaben ein Beschäftigter zu erfüllen hat, desto sicherer und qualifizierter kann er diese ausüben. Gerade in der Anlaufphase eines Betriebs erweist es sich daher als vorteilhaft für das Management, unerfahrenen Arbeitern einen nur begrenzten Funktionsraum anzuvertrauen.

Allerdings weisen schon Mickler et al. (1976) darauf hin, dass dieses Kalkül längerfristig nicht aufgeht, sondern zu einem kostspieligen Nachteil werden kann: Das durchschnittliche Qualifikationsniveau ist dann zwar niedrig und damit „billiger“ zu erhalten, aber der Personalstand muss in Ermangelung flexibler Personaleinsatzstrategien dafür vergleichsweise hoch sein. Mickler et al. führen dies auf die speziellen Anforderungen der Arbeit an komplexen, automatisierten Maschinen zurück und sehen entscheidende Nachteile dieser strikten Arbeitsteilung. ${ }^{97}$ Dass die Arbeitsgestaltung in der Chemische Industrie trotz aller Dysfunktionalitäten durch ein hohes $\mathrm{Maß}$ an arbeitsorganisatorischem Konservatismus im Hinblick auf die Zuordnung von Innovationskompetenz gekennzeichnet ist, unterstreicht die Beharrlichkeit der charakteristischen Wissensbasierung. Wo Produktvielfalt und Produkt- wie Verfahrensinnovationen die strategische Ausrichtung bestimmen, und die Vorrangstellung in Forschung und Entwicklung die Marktposition sichert, steht eine Modifikation des Produktionsmodells in punkto Arbeitsorganisation auf dem Shop floor nicht auf der Prioritätenliste.

Verstärkend wirken in diesem Prozess die arbeitspolitischen Konzeptualisierungsprobleme der betrieblichen Interessenvertretung, die in hohem Maß der Branchenspezifität geschuldet sind. ${ }^{98}$ Die starke Position der Einzelbetriebe erforderte eine Basisnähe, die mittels der beschränkten personellen Ressourcen nicht einzulösen ist, und die eine Vertretung der Belegschaften im Hinblick auf arbeitsorganisatorische Fragen nahezu unmöglich macht. ${ }^{99}$ So überwiegt bei den Betriebsräten in der Chemischen Industrie ein eher manageriell-selektives Rollenverständnis (Kern, Schumann 1990:288), das eine Fokussierung der Betriebsratsarbeit auf Einzelaspekte impliziert. Schwerpunkte sind Problemfelder im Bereich von Arbeit, die auf oberster Managementebene reguliert werden oder aber sozialmanagerieller Art sind: Lohn-, Sozial-, Arbeitsmarkt-, Ausbildungs-, Arbeitszeitpolitik

\footnotetext{
${ }^{97}$ Mickler et al. verweisen auf folgende Probleme : Jeder Arbeitsbereich benötigt eigene Personalreserven, um Krankheit oder Urlaub auszugleichen. Die zeitlich überwiegende Handlungssituation der passiven Arbeitsanteile führt dann dazu, dass die Beschäftigten tatsächlich „nur“ auf eine Störung innerhalb ihres Anlagenteils warten, für das Unternehmen somit Zeitreserven ungenutzte bleiben. Einzelne Beschäftigte sind nicht in der Lage, ihren Kollegen Hilfe zu leisten, denn an anderen Stellen als an ihrer Anlage sind sie nicht einsatzfähig. Je länger der Betrieb läuft, desto geringer wird die Anzahl der Störungen. Der Erhalt der eigenen ohnehin schon beschränkten Qualifikation wird zunehmend in Frage gestellt. Durch die Reduzierung der Sicht auf einen Ausschnitt des Prozesses verliert der Anlagenfahrer den Überblick über den Gesamtablauf. Dies kann zu erheblichen Fehleinschätzungen und Folgefehlern in Extremsituationen führen (Mickler et al. 1976:271).

${ }^{98}$ Es sei an dieser Stelle noch einmal ausdrücklich darauf verwiesen, dass wir uns auf die Interessenvertretung in Großunternehmen beziehen, die klein- und mittelständischen Betriebe agieren unter anderen Bedingungen.

${ }^{99}$ Diese Distanz zur einzelbetrieblichen Realität spiegelt sich auch im extrem niedrigen Organisationsgrad wider (Hertle, Kädtler 1990).
} 
sowie die Verwaltung betrieblicher sozialer Einrichtungen. In diesen Bereichen agiert die Interessenvertretung zumeist als Co-Management, und die Branche ist durch eine harmonische „sozialpartnerschaftliche“ Beziehung zur Unternehmensleitung charakterisiert (Schudlich 1982). Die Betriebsräte versuchen, in enger Zusammenarbeit mit dem Management die sozialen Aspekte des Faktor Arbeit in den Managemententscheidungen zu stärken. Bedingt durch die äußerst heterogenen Arbeitstrukturen hat sich die Interessenvertretungspolitik den Themen Produktionstechnik und Arbeitsstrukturen aber fast ausschließlich unter dem Aspekt des Arbeitsschutzes und der Arbeitssicherheit genähert. Diese bestimmen als relevante Aktionsfelder in weiten Teilen die Arbeit, und in Anbetracht der Gefährdungslagen hatte dies auch seine Berechtigung. Bezogen auf die konkreten Arbeitsstrukturen und das in ihnen liegende, über sicherheitsrelevante Aspekte hinausreichende arbeitsgestalterische Potential ist die Politik der Interessenvertretung bis Ende der 1980er Jahre aber einigermaßen konzeptionslos. Zwar sehen auch die Betriebsräte das Problem des unterqualifizierten Einsatzes der Chemikanten. Doch die Erfahrungen mit dem produktionstechnischen Wandel, d.h. die Entlastung von körperlich anstrengender Arbeit und gefährlichen Umwelteinflüssen sowie die qualifikatorisch anspruchvolleren Tätigkeitszuschnitte in den Messwarten verstärken die Einschätzung, dass die technische Entwicklung auch facharbeitergerechte Arbeitsplätze nach sich ziehen werde (Schumann et al. 1994).

Dieser arbeitspolitische Konservatismus wird durch eine betriebsratsinterne Konfliktlinie gestützt. Die inhaltlich-arbeitsorganisatorisch abgesicherte Stärkung des Produktionsfacharbeiters würde mit einer Ausweitung technisch-handwerklicher Funktionen einhergehen. Besitzstände der Instandhaltungsbereiche würden so zur Disposition stehen, eine harte Belastungsprobe für die Interessenvertretung. So konstatieren auch Kern und Schumann:

„Wenn, animiert durch den Facharbeiter in der Produktion, der betriebliche Zugriff auf
den Instandhaltungsbereich tatsächlich stattfindet, wird es für die Chemiebetriebsräte hei-
kel werden. Sie werden sich überlegen müssen, wie sie gegenüber einem solchen Konzept
eine Position entwickeln, mit der sie am Ende nicht zwischen allen Stühlen sitzen.“ (Kern,
Schumann 1990:299)

Das Austarieren von Interessen der Produktionsarbeiter auf der einen Seite und Instandhaltungsarbeiter auf der anderen dürfte, vergegenwärtigt man sich noch einmal das Statusmodell der Industrie, mit erheblichen Schwierigkeiten verbunden sein. Und so könnte ein Teil dessen, was nach außen als arbeitspolitischer Konservatismus der Betriebsräte interpretiert werden kann, auf die interne Zerrissenheit der Interessenvertreter verweisen. Die Instandhaltungsbereiche gelten in den Betriebsräten und Gewerkschaften als äußerst einflussreiche politische Kraft, sie zu Mitspielern einer solchen Arbeitspolitik zu machen, die ihre Position schwächen könnte, dürfte nicht einfach sein.(Schumann et al.1994; Kädtler 1997). 


\section{Entwicklung des Innovations- und Produktionsmodells in den 1990er Jahren}

Die ökonomische Bilanz der deutschen Chemischen Industrie liest sich Ende der 1980er Jahre recht positiv. Die auf Qualitätsprodukte setzende Innovationsstrategie der Unternehmen bewährt sich, und es steigen sowohl die Kapazitätsauslastung wie die Anzahl der Beschäftigten. Zugleich kann das komplexer wie komplizierter gewordene Verwertungskonzept der gestiegenen Relevanz von Energie- und Rohstoffpreisen, Umweltbelastungen, Produktqualität, Prozesssicherheit und Flexibilität in hohem Maß Rechnung tragen. Ihre Rolle als prägender gesamtwirtschaftlicher Wachstumsmotor verliert die Branche allerdings, und so kann die Chemische Industrie zwar weiterhin mit Rekordgewinnen aufwarten, diese basieren jedoch im Vergleich zu anderen Branchen auf einem eher maßvollen Produktions- und Umsatzwachstum (Schumann et al. 1994; VCI 1990). Erst die gesamtwirtschaftliche Krise in den Jahren 1992 und 1993 trifft die Chemische Industrie und führt zur schwersten Konjunkturkrise der Nachkriegszeit, mit erheblichen Konsequenzen für die Beschäftigungsentwicklung: Zwischen 1991 und 2001 sinken die Beschäftigungszahlen ${ }^{100}$ von 593.816 auf den historischen Tiefstand ${ }^{101}$ von 434.789 (BAVC 2002) und der Anteil der Beschäftigten im gewerblichen Bereich schrumpft in den Jahren 1993 bis 1996 überdurchschnittlich stark.

\begin{tabular}{|l|l|l|l|l|}
\hline \multirow{2}{*}{} & \multicolumn{2}{|c|}{ Beschäftigte (gesamt) } & \multicolumn{2}{c|}{ Arbeiter } \\
\cline { 2 - 5 } & In 1000 & Veränderung in \% & In 1000 & Veränderung in \% \\
\hline 1990 & 583,3 & & 293,6 & \\
\hline 1991 & 584,4 & 0,2 & 291,2 & $-0,8$ \\
\hline 1992 & 577,1 & $-1,3$ & 283,1 & $-2,8$ \\
\hline 1993 & 543,6 & $-5,8$ & 258,9 & $-8,5$ \\
\hline 1994 & 521,8 & $-4,0$ & 245,4 & $-5,2$ \\
\hline 1995 & 500,6 & $-4,1$ & 232,8 & $-5,1$ \\
\hline 1996 & 485,9 & $-2,9$ & 223,3 & $-4,1$ \\
\hline 1997 & 468,5 & $-3,6$ & 214,9 & $-3,8$ \\
\hline 1998 & 453,3 & $-3,2$ & 207,3 & $-3,5$ \\
\hline 1999 & 446,7 & $-1,5$ & 201,5 & $-2,8$ \\
\hline
\end{tabular}

Quelle: Produktion und Faktoreneinsatz nach Branchen des verarbeitenden Gewerbes in Westdeutschland, DIW 2000

Tabelle 1: Beschäftigungsentwicklung in der Chemischen Industrie 1990-1999

\footnotetext{
${ }^{100}$ Grundlage sind entsprechend der Fokussierung dieser Arbeit auf die westdeutsche Chemische Industrie die Zeitreihen für Westdeutschland. Der Beschäftigungsabbau nimmt sich unter Einbezug der gesamtdeutschen Daten noch umfassender aus, hier sinken die Beschäftigungszahlen im Vergleichszeitraum von 716.734 auf 467.029 (VCI 2002).

${ }^{101}$ Nach dem Zweiten Weltkrieg erreicht die westdeutsche Chemische Industrie im Jahr 1974 mit 601.600 ihren höchsten Beschäftigungsstand, sowohl davor als auch danach pendelte sie im Bereich von 550.000, erst seit 1991 ist ein relevanter Personalabbau zu konstatieren (vgl. auch http//:bavc.de).
} 
Diese Entwicklungen stehen allerdings für mehr als Rationalisierung durch Stellenabbau. Zwar wird die hohe Kapitalintensität bedingt durch neue Prozesstechnologien in den 1990er Jahren auch rationalisierungswirksam im Hinblick auf Beschäftigung. Doch die schon 1994 einsetzende positive Umsatzentwicklung (+7,5 Prozent), die sich bis ins Jahr 2000 (+11,5 Prozent) fortsetzt, steht für einen viel tiefgreifenderen Strukturwandel, den die Unternehmen der Chemischen Industrie in den 1990er Jahren eingeleitet haben. ${ }^{102}$

Dieser manifestiert sich im besonderen im strategisch traditionell hoch relevanten Bereich der FuE. Auch hier kommt es, wie in anderen Unternehmensbereichen, zu erheblichen Personaleinsparungen, dass FuE-Personal wird von 61.336 (1991) auf $44.158 \mathrm{im} \mathrm{Jahr}$ 1999 (VCI 2003) abgebaut. Parallel dazu werden in der ersten Hälfte der1990er Jahre die Investitionen für FuE zunächst erheblich gekürzt, doch schon 1995wird ein Aufwärtstrend eingeleitet, und mit einer Steigerung des Budgets um 13,1 Prozent erreichen die FuEAufwendungen mit knapp sieben Milliarden Euro einen historischen Spitzenwert.

\begin{tabular}{|l|l|l|l|l|l|l|}
\hline & \multicolumn{2}{|c|}{$\begin{array}{c}\text { FuE-Aufwand } \\
\text { in Mio. DM }\end{array}$} & \multicolumn{2}{c|}{$\begin{array}{c}\text { gesamtes } \\
\text { FuE-Personal }\end{array}$} & \multicolumn{2}{c|}{$\begin{array}{c}\text { Wissenschaftler } \\
\text { in FuE }\end{array}$} \\
\hline 1990 & 10.650 & $+3,6$ & 56.300 & $-2,1$ & & \\
\hline 1991 & 10.948 & $+2,8$ & 61.336 & $+8,9$ & 16.873 & \\
\hline 1992 & 10.636 & $-2,8$ & 56.451 & $-8,0$ & 14.813 & $-12,2$ \\
\hline 1993 & 10.547 & $-0,8$ & 53.306 & $-5,6$ & 13.400 & $-9,5$ \\
\hline 1994 & 10.095 & $-4,3$ & 49.050 & $-0,8$ & & \\
\hline 1995 & 10.446 & $+3,5$ & 49.081 & 0,0 & 12.743 & \\
\hline 1996 & 11.289 & $+8,1$ & 49.768 & $+1,4$ & & \\
\hline 1997 & 12.045 & $+6,7$ & 47.297 & $-5,0$ & & \\
\hline 1998 & 13.618 & $+13,1$ & 48.970 & $+3,5$ & 12.560 & \\
\hline 1999 & 14.270 & $+4,8$ & 48.190 & -16 & & \\
\hline
\end{tabular}

VCl 2001, 97

Tabelle 2: Forschungs- und Entwicklungsaktivitäten in der Chemischen Industrie

Neben dem Beschäftigungsabbau und der Erhöhung der FuE-Ausgaben stellt eine strategische Neuausrichtung der Forschungslinien die dritte Säule des strukturellen Wandels. Insbesondere die großen integrierten chemisch-pharmazeutischen Unternehmen machen in der zweite Hälfte der 1990er Jahre Schlagzeilen durch teils spektakuläre Fusionen, Verkäufe und den Auf- und Ausbau internationaler Standorte (Zeller 2001). Retrospektiv scheinen die Chemieunternehmen mit einigem Erfolg ihr Innovations- und Produktionsmodell umgebaut zu haben (Bathelt 1997b; Becker 2001). Diese Entwicklung ist insofern bemerkenswert, als die Bedingungen am Weltchemiemarkt Ende der 1980er Jahre denkbar ungünstig für die Chemiekonjunktur waren und eine „Innovationslücke“ die Unter-

${ }^{102}$ Der Beschäftigungsabbau im Inland ist auch als Konsequenz weitreichender Umstrukturierungsmaßnahmen der Chemieunternehmen zu verstehen. So wird ein Teil des Rückgangs durch Outsourcingprozesse erklärt. 1998 etwa fallen 15.000 Beschäftigte aus der Statistik der Chemischen Industrie, da ihre Abteilungen im Rahmen der Reorganisation eines Unternehmens zu selbständigen nicht-chemischen Firmen umdefiniert werden (VCI 2000). Das BMBF schätzt den realen Beschäftigungsrückgang für das Jahr 1998 lediglich auf ein Prozent, während der VCI von 3,2 Prozent ausgeht (BMBF 1999; VCI 2002). 
nehmen zusätzlich unter erheblichen Druck setzte. Wissen und damit verbunden Produkte und Verfahren veralteten immer schneller, d.h. die Lebensspannen der Produkte verkürzten sich, und bei weltweit verschärfter Konkurrenz stiegen die Kosten für FuE. Für eine Erfolgsquote auf der Produktseite musste das innovative Potential erheblich gesteigert werden, doch sinkt die innovative Stärke der Branche gerade in den 1980er Jahren deutlich. Immer weniger neue chemische Moleküle werden entwickelt, immer seltener werden existierende Grundstoffe durch qualitativ neue substituiert. ${ }^{103}$ Dies ist umso gravierender, als sich gleichzeitig die Investitionen in FuE zwischen 1980 und 1991 verdoppeln und bezogen auf den Branchenumsatz von 4,2 (1980) auf 5,6 Prozent (1991) steigen (VCI 1992). Insbesondere die pharmazeutischen Unternehmen sowie die Pharmasparten der integrierten Unternehmen ${ }^{104}$ sind von der Innovationslücke betroffen und zwar in doppelter Weise: Nicht nur sinkt die Ausbringung an innovativen Wirkstoffen, zugleich steigen auch seit den 1970er Jahren aufgrund immer aufwändigerer Zulassungs- und Testverfahren die Kosten für die Arzneimittelentwicklung exponentiell (Drews 1998). ${ }^{105}$ Alles in allem scheinen entscheidender für die günstige Gewinnentwicklung der 1980er Jahre nicht so sehr die steigenden FuE-Anstrengungen, sondern die rückläufigen Rohstoffeinstandskosten sowie verbesserte Kapazitätsauslastungen der kapitalintensiven Produktionsanlagen zu sein, die zu Kostendegressionseffekten führen (Schumann et al. 1994).

Um die Strategie - und Strukturentwicklungen der Chemieunternehmen vor diesem Hintergrund zu analysieren, ist ein komplexes Feld zu entschlüsseln, in dem branchenspezifische wie branchenübergreifende Bedingungen und Trends zusammenwirken (Becker 2001). ${ }^{106}$ Als solche gelten etwa technische Innovationen, die Nachfrageentwicklung, die

\footnotetext{
${ }^{103}$ Wöcherl (1989) kommt zu einer diametral entgegengesetzten Einschätzung der Situation und verweist darauf, dass gerade die Konzentration der Produktionserweiterungen bei Spezialitäten Ausdruck der gesteigerten FuELeistungsfähigkeit sei. Betrachtet man den Anteil der Spezialchemie am Gesamtvolumen der FuEAufwendungen, so wird nach wie vor etwa die Hälfte an FuE in chemische Grundstoffe investiert, es folgt der pharmazeutische Sektor und erst an dritter Stelle liegt die Spezialchemie. Die von Wöcherl diagnostizierte ,stark erhöhte Neuentwicklungsproduktivität“ der FuE ist von daher eher programmatische Ansage denn durch Zahlen fundiert. Gerade Wöcherls Prognosen für den Pharma- und Pflanzenschutzsektor sind getragen von einer - wie heute resümierend festgehalten werden kann - allzu euphorischen Einschätzung der Möglichkeiten der neuen Biotechnologien. Insgesamt zieht Wöcherl den für die Chemische Industrie falschen Schluss, dass ein Mehr an Innovationsaufwendungen auch eine steigende Innovationsrate nach sich zieht. Dieser Zusammenhang ist allerdings nicht aufrecht zu erhalten (Drews 1998).

${ }^{104}$ In Deutschland hat die Pharmasparte insgesamt einen Anteil von etwa $20 \%$ an der Produktion von chemischen Erzeugnissen. Die Zahl der Beschäftigten liegt bei 132000.

105 Zahlen über die Kosten für Wirkstofffindung und Entwicklung von Arzneimitteln beinhalten immer auch die Aufwendungen für Fehlschläge. Es kann also davon ausgegangen werden, dass der Anstieg von ca. 24,4 Millionen Dollar, der für die 1950er und 60er Jahre veranschlagt wird auf 359 Mio. Dollar 1990 bis auf heute ca. 800 Mio Dollar (Drews 1998:230) auch ein Ausdruck des Reifestadiums der Wissenschaftsentwicklung ist. Wir werden später auf diesen für die FuE wesentlichen Punkt zurückkommen.

${ }^{106}$ Wir benutzen den unscharfen Begriff „entschlüsseln“ an dieser Stelle um anzuzeigen, dass es sich bei der Analyse der Strategie- und Strukturentwicklungen um ein „Phänomen organisierter Komplexität“ (Baecker 1999:30) handelt, das weder einfach noch unorganisiert ist. Für Phänomene dieser Art (Beispiele hierfür sind neben Unternehmen etwa der Aufbau von Atomen, chemische Verbindungen, oder auch Familien und Gesellschaft) für die die Standardmethoden der wissenschaftlichen Beschreibung (kausale Erklärung und falsifizierbare Vorhersage) nicht mehr anwendbar sind. „Phänomene organisierter Komplexität basieren auf der Gleichzeitigkeit aller wesentlichen Abläufe, und Gleichzeitigkeit schließt Kausalität aus. Vorhersagen sind nicht zu treffen, weil Phänomene organisierter Komplexität ausschließlich auf der Basis einer undurchschaubaren Eigengeschichte operieren.“ (Baecker 1999:30) Im folgenden wird der Versuch unternommen, diese Gleichzeitigkeit aufzunehmen und in Zusammenhang zu setzen mit den aktuellen Entwicklungen, ohne dass sich dabei einzelne Trends kausal
} 
Konkurrenzsituation sowie spezifische staatliche Regulierungen, aber auch die Revolutionierung der Informations- und Kommunikationstechnologien, billigere und effizientere Transporttechnologien, die Internationalisierung der Finanzmärkte und der weltweite Abbau von Handelsbarrieren. Die einzelnen Bedingungen können strategische Ausrichtungen von Unternehmen insofern beeinflussen, als sie als dynamisches Verstärkungsarrangement für den Wandel von Innovations- und Produktionsmodell verstanden werden, ohne diesen zu determinieren (3.1). Dabei stellt sich insbesondere die Frage, wieweit und in welchen Dimensionen die Chemische Industrie als science based industry in besonderer Weise von den zu beobachtenden Transformationen und Re-Arrangements der industriellen Produktion beeinflusst ist (3.2), und welche Wandlungsprozesse auf der Ebene des Innovationsmodells der Chemischen Industrie zu beobachten sind (3.3). Darüber hinaus fragen wir danach, in welche Richtung sich Anforderungsprofile für Wissenschaftler und Ingenieure verändern und welche Ausstrahlungseffekte sich aus den analysierten Wandlungsprozessen für Arbeitsorganisation und Arbeitskraft auf dem Shop floor ergeben (3.4).

\subsection{Von der verbund- zur portfolioorientierten Unternehmensorganisation}

In Kapitel 2 haben wir bereits auf das technisch-organisatorische Paradigma der Verbundchemie, das die deutsche Chemische Industrie in besonderer Weise prägt, ${ }^{107}$ hingewiesen. Der in den 1970er Jahren einsetzende Rückzug aus dem Massengeschäft der deutschen Chemieunternehmen und die Fokussierung auf höherwertige Produkte änderten daran wenig, und insgesamt bleiben die Chemieunternehmen durch ein hohes Maß an vertikaler wie horizontaler Integration gekennzeichnet. Die damit verbundene ökonomische Strategie fokussiert auf die Optimierung und Weiterentwicklung der komplexen Produktionskompetenz unter Berücksichtigung eines heterogenen Produktspektrums, neu zu entwickelnder Produkte und zu erschließenden Märkten.

Bei einer relativ ausgewogenen Entwicklung einzelner Geschäftsfelder stellt dies kein Problem dar, doch ein Blick auf die Struktur der deutschen Chemiewirtschaft belegt, dass es für einzelne Sektoren in den vergangenen Jahrzehnten teils erhebliche Umbrüche gegeben hat. ${ }^{108}$ So wuchs etwa die Sparte chemische Spezialitäten zwischen 1978 und 1989 um 26 Prozent, während im Vergleichszeitraum der Anteil an anorganischen Industrieprodukten um zwei, die Pflanzenschutzmittelproduktion um beinah 10 Prozent sank. Und schließlich sinkt der Anteil an Grundchemikalien ${ }^{109}$ (Anorganica und Organica) zwischen

bedingen. Dass dies insbesondere auf der Ebene der sprachlichen Darstellung nicht immer gelingt, ist ein im Rahmen dieser Arbeit berücksichtigtes, aber nicht immer zu lösendes Problem.

107 Das aus den chemischen Prozessen resultierende Entsorgungsproblem führt erstaunlicher Weise nur in Deutschland zur Verbundchemie. Kädtler (2003) verweist darauf, dass sich darin eine vor allem von naturwissenschaftlichen und ingenieurtechnischen Gesichtspunkten bestimmte Lösungsperspektive niederschlägt. Ob dies wiederum mit der besonders ausgeprägten Anbindung der ersten deutschen Chemiefirmen an die Wissenschaft zusammenhängt, kann mangels einschlägiger Studien nur vermutet werden.

${ }^{108}$ Die Datenbasis für die folgenden Ausführungen sind, soweit nicht anders ausgewiesen, verschiedene Jahrgänge der vom VCI publizierten Broschüre „Chemiewirtschaft in Zahlen“.

109 Als Grundchemikalien oder Grundstoffchemie werden die ersten Verarbeitungsstufen nach der Petrochemie bezeichnet. Sie umfasst eine relativ geringe Anzahl an hochvolumigen Produkten. Sie ist äußerst kapitalintensiv und hängt in ihrer Entwicklung stark von Rohstoffpreisen ab. Bei Innovationen steht die Prozessoptimierung im Mittelpunkt. 
1984 und 1993 von 29,7 auf 20,5 Prozent. Alles in allem driftet die Entwicklung einzelner Geschäftsbereiche immer eklatanter auseinander. Während auf der einen Seite die Pharmasparten durchgängig aller großen Chemieunternehmen in der Regel zweistellige Gewinne schreiben, müssen auf der anderen Seite die Bereiche Kunststoffe und Fasern hohe Verluste hinnehmen, Bereiche wie Polymere und Organica fahren eher unterdurchschnittliche Renditen ein (Schumann et al. 1994).

Mit dieser Entwicklung werden die für die deutsche Chemische Industrie lange Zeit gültigen technisch-ökonomischen Vorteile der historisch gewachsenen Verbundproduktion zunehmend in Frage gestellt. Der über weite Strecken integrierte Prozesscharakter der Fertigung konzentrierte die Wertschöpfungsaktivitäten an den traditionellen Standorten. Energieverbund, Transportrisiken, Qualitätssicherung sowie die Verkettung der automatisierten Produktionsabläufe konnten so optimal miteinander kombiniert werden. Durch Massenproduktion im Rahmen vollkontinuierlicher Produktionsprozesse können bei der Herstellung anorganischer wie organischer Grundchemikalien in hohem Maß economics of scale (Chandler 1990) genutzt werden. Die bei diesen Prozessen anfallenden meist umweltschädlichen Stoffe werden in nachgeschalteten Produktionsprozessen weiter verarbeitet, was eine effektive Alternative zum Transport ist. Sowohl der Wirtschaftlichkeit wie auch den seit den 1970er Jahren gestiegenen ökologischen Anforderungen wird die Verbundproduktion damit gerecht. Verändern sich Markt- und Wettbewerbssituation nicht konjunkturell bedingt sondern strukturell, wie dies seit nunmehr drei Jahrzehnten in der Chemischen Industrie geschieht, kommt es innerhalb eines Verbundsystems zu negativen oder positiven Rückkopplungen, die eine modifizierte Verbundstruktur erfordern. Dies betrifft insbesondere den Wandel der Branche im Geschäftsfeld der Grundchemikalien. Im Unterschied zu anderen Sparten herrscht hier bei geringen Gewinnmargen ein intensiver Preis- und Kostendruck. Zunehmend können Zwischenprodukte preisgünstiger auf dem Weltmarkt erstanden werden als bei einheimischen Herstellern bzw. implizieren Importe weniger Kosten als die Herstellung im eigenen Verbund. Eine dauerhaft geringere Kapazitätsauslastung hat dann jedoch nicht nur Konsequenzen für die Herstellung der Zwischenprodukte, sondern senkt die Nachfrage nach Grundstoffen. Langfristig wird so das Verbundsystem insgesamt in Frage gestellt, denn je mehr sich das Produktspektrum auf hochveredelte Stoffe fokussiert, desto ökonomisch fragwürdiger wird die Verbundproduktion (Bathelt 1997).

Die Spezialisierung und Heterogenisierung der Produktions- und Kostenstruktur ist von daher als ein Movens der partiellen Ablösung horizontal und vertikal integrierter Strukturen in der Chemischen Industrie zu verstehen (Koubek 1996; Menz et al. 1999). Seit Beginn der 1990er Jahre überwinden die Großunternehmen der Chemischen Industrie verstärkt die verbundtechnologisch bedingten Anforderungen. Die Produktionsabläufe werden entsprechend technologischer Möglichkeiten neu geordnet, d.h., durch verfahrenstechnische Änderungen bei der Herstellung und Integration von Vorprodukten können wirtschaftliche Aspekte auch bei mehrfacher Unterbrechung des Produktionsprozesses berücksichtigt werden. Die daraus resultierende Form des Produktionsablaufs wird als ,Komponentenchemie' bezeichnet. Koubek et al. folgern daraus, dass die Chemische Industrie damit weitgehend ihren bisherigen „technologiebedingten Sondercharakter“ verliere und sich anderen Branchen angleiche (Koubek 1996:27). 
Inwiefern sich in der vorhergehenden Phase tatsächlich technologiebedingt das Verbundsystem als einzig wirtschaftliche Struktur für die Großunternehmen der Chemischen Industrie darstellte, soll an dieser Stelle nicht weiter diskutiert werden. Fest steht, dass die Produktion transnational auf neuartige Weise vernetzt und optimiert wird. Das erhöhte internationale Engagement der ohnehin seit langem international agierenden Chemischen Industrie vermittelt sich insbesondere über die wachsenden Direktinvestitionen von Unternehmen im Ausland. Diese haben sich in den letzten zwei Jahrzehnten vervierfacht und seit Anfang der 1990er Jahre ist in diesem Segment ein besonders dynamisches Wachstum zu verzeichnen. So investierten zwischen 1990 und 1994 die Chemieunternehmen 20 Milliarden Euro in ausländische Sachanlagen, dies entspricht einem Anteil von etwa 64 Prozent der Summe, die im Inland aufgewendet wird. In der zweiten Hälfte der 1990er Jahre stieg dieser Anteil auf knapp 87 Prozent, mit 28 Milliarden Euro lagen die Auslandsinvestitionen in diesem Zeitraum damit um circa 40 Prozent höher als in den fünf Jahren zuvor (VCI 2002). Geographisch gesehen verstärken die Firmen mit gut der Hälfte der Gesamtinvestitionen vor allem ihre Präsenz in der Nordamerikanischen Freihandelszone (NAFTA), ein Viertel entfiel auf Westeuropa, etwa 20 Prozent der ausländischen Sachanlageinvestitionen wurden im asiatischen Raum getätigt. Der Investitionsschub deutscher Chemieunternehmen im Ausland ist Ausdruck einer - relativen - Standortverlagerung: Es wird absatznäher produziert, und Kostenvorteile werden genutzt. Die stofflichfunktionale Seite der Produktion hat in diesem Prozess einen abnehmenden, aber nicht obsoleten Einfluss auf die Strategiewahl der Unternehmen. Standardproduktionen, die technologisch robuster und im Hinblick auf Standortinfrastruktur und Arbeitskräftequalifikation anspruchsloser sind, werden in der Peripherie und Semiperipherie der Triade angesiedelt (Kädtler 2000).

Ihren organisatorischen Ausdruck finden diese Dezentralisierungsprozesse meist in einer Divisionalisierung in Unternehmensbereiche unterhalb der Konzernspitze oder Geschäftsleitung und zwar gegliedert nach Produkten, Produktgruppen oder Kundengruppen. Die interne Organisation der Divisionen erfolgt sehr unterschiedlich und kann zwischen einer klassischen funktionalen Gliederung mit einer recht engen Bindung an die strategische Spitze bis zur rechtlichen Selbständigkeit und der Ausübung unternehmerischer Handlungen in Form von Management- und Holdingsstrukturen reichen.

Die Unternehmen überprüfen dabei ihre Produktionsketten verstärkt auf Spezialisierungsvorteile und versuchen, über die Definition von Kernkompetenzen ${ }^{110}$ und strategischen Geschäftsfeldern ${ }^{111}$ ihre Produktportfolios zu optimieren. ${ }^{112}$ Eine wesentliche Rolle spielt

\footnotetext{
${ }^{110}$ Als Kernkompetenzen sind der Definition von Hamel und Prahalad folgend die wesentlichen technischen, technologischen, vertrieblichen und organisatorischen Fähigkeiten eines Unternehmens zu verstehen. (Hamel, Prahalad 1995, zitiert nach Picot et al. 1998).

111 Strategische Geschäftsfelder bezeichnen definierte Marktleistungsangebote in bestimmten Marktsegmenten (Wiendahl 1997).

${ }^{112}$ Aus Gründen der Risikominimierung sind Unternehmen meist in mehreren Feldern und Regionen tätig. Ziel der Unternehmensführung ist es, ein Gleichgewicht zwischen Geschäftseinheiten mit hohem und niedrigem Risiko zu gewährleisten. Die ursprünglich zur Anlage von Wertpapieren entwickelte Portfolio-Technik hat sich in den letzten Jahren durchgesetzt und verweist auf die größere Relevanz der klassischen Finanzinvestmenttheorie: Die einzelnen Geschäftsfelder werden anhand ihres jährlichen prozentualen Marktwachstums und ihres Marktanteils entsprechend in einer Vierfelder-Matrix positioniert, die zur Analyse und Entwicklung aller Felder des Unternehmens genutzt wird (Ötinger 1994).
} 
bei diesen Überlegungen seit Beginn der 1990er Jahre das in den 1980er Jahren in den USA entwickelte Konzept des Shareholder Value und die damit verbundenen Strategien der kapital- bzw. finanzmarktorientierten Rationalisierung. Diese basiert auf der Grundüberlegung, dass Finanzkapital im Rahmen der kapitalistischen Produktionsweise ein Doppelcharakter inhärent ist. Es ist einmal von der Bewegung des industriellen Kapitals abhängig, andererseits hat es in Form des fiktiven Kapitals eine eigenständige Bewegungsform, die sich von der industriellen Grundlage weitgehend abheben kann (Menz et al. 1999:34f.). Dahinter steht die Überlegung, dass Kapital definiert werden kann als „Ausbeutung“ von Lohnarbeit, wie auch als Verwandlung von Geld in mehr Geld. Von fiktivem Kapital kann immer dann die Rede sein, „wenn der zweite Teil der Definition den ersten nicht mehr enthält, und zwar nicht etwa als bloß subjektive Einbildung, sondern als gesellschaftliche Realität“"113. Neben den kapitalistischen Waren- und Arbeitsmärkten existiert entsprechend dieser Definition noch ein Finanz- oder GeldkapitalMarkt. Die Möglichkeit des fiktiven Kapitals ist auf letzterem gegeben mit dem zinstragenden Kapital des Kreditsystems. Dieses wird nicht direkt als Produktivkapital benutzt, sondern an anderes Kapital für diesen Zweck verliehen, wofür in Gestalt des Zinses ein Anteil vom Mehrwert bzw. Profit des Realkapitals abfällt. Auf diesem können aber auch Aktien zu fiktivem Kapital werden, wenn die realen Unternehmensgewinne etwa entkoppelt von den Bewertungen auf den Aktienmärkten verlaufen. Insbesondere in der zweiten Hälfte der 1990er Jahre werden vor allem die Chemieunternehmen an den Aktienmärkten hoch eingestuft, die einen Schwenk auf das zukunftsträchtige Feld der Life Sciences vornehmen. Unabhängig von der Etablierung „echter“ Produktinnovationen reichte allein die Ankündigung bestimmter Forschungsthemen für diese Aufwertung aus. Dies forciert die Konzentration der Konzerne auf die übergreifende Kombination aus den Sparten Landwirtschaft und Pharma.

Die Unternehmen setzen in dieser Phase auf eine Neuordnung der Verwertungsprioritäten und versuchen, einen Ausgleich zwischen Finanz- und Produktionsinteressen zu finden. Die deutschen Chemiekonzerne reagieren auf den verstärkten Wettbewerbsdruck in forschungs- und entwicklungsintensiven Sparten, auf die Sättigung zahlreicher traditioneller Märkte und auf Wachstumschancen in neuen, kapitalintensiven mit einer variantenreichen Reorganisation und Verschlankung. Der Diversifikationstrend früherer Jahre weicht einer Konzentration auf profitable, konjunkturrobuste und innovative Geschäftsfelder. Dabei ist die Frage, wie viel stofflicher Koordinationsbedarf besteht und wie weit er im einzelnen reicht, nicht eindeutig zu beantworten. Beispielhaft dafür stehen die unterschiedlichen Strategien von Akteuren in Unternehmen (Becker 2001), die sich auch empirisch nachweisen lassen und unter diesen Bedingungen stark voneinander abweichen. ${ }^{114}$

\footnotetext{
${ }^{113}$ vgl. Trenkle, Kurz : « Fiktives Kapital » unter : www.krisis.org/n-trenkle_fiktiveskapital.html

${ }^{114}$ Die Kurzdarstellungen stellen ein Destillat aus Geschäftsberichten einzelner Jahrgänge, den Internetportalen der Unternehmen sowie den Veröffentlichungen von Dolata 1997, Koubek 1997, Kädtler 2000 sowie Becker 2001 dar.
} 
BASF - Verbund bleibt die strategische Ausrichtung

Das Management der BASF setzt nach wie vor auf die traditionelle Verbundchemie, mit diesem Konzept wird in der Selbstdarstellung ${ }^{115}$ offensiv geworben und das ,alte Realitätskonzept“ so bekräftigt.

„Die BASF ist ein ertragsorientiertes transnationales Unternehmen, das besonders in den Bereichen Chemie, Gesundheit und Ernährung sowie Öl und Gas wertsteigerndes Wachstum anstrebt. Die Produktpalette des Unternehmens umfasst hochveredelte Chemikalien, Kunststoffe, Farbstoffe, Dispersionen, Fahrzeug- und Industrielacke, Pflanzenschutzmittel, Pharmazeutika, Feinchemikalien, Öl und Gas. Die ausgeprägte Verbundstrategie ist eine der besonderen Stärken der BASF und bietet dem Unternehmen entscheidende Wettbewerbsvorteile.“" ${ }^{\text {116 }}$

Die Verbundtechnologie bleibt die Grundlage für Entscheidungen im Bereich der Steuerung, der Organisation sowie der Ertragsrechnung. Allerdings findet eine Anpassung an die verschärften Marktbedingungen auch bei BASF ihren Ausdruck im Einsatz moderner Controllingstrategien, dem größeren Gewicht von Wirtschaftlichkeitskriterien bei der Beurteilung verschiedener Geschäftsfelder, der Integration indirekter Funktionen in die Geschäftsbereiche sowie der Ausgründung wenig effizienter Bereiche. Außerdem stärkt die BASF ihre internationalen Standorte durch strategische Allianzen sowie Joint Ventures mit anderen Chemiekonzernen ${ }^{117}$ und kleineren High Tech Unternehmen ${ }^{118}$. Die Forschung konzentriert sich auf den Standort Deutschland, speziell das Stammhaus in Ludwigshafen. Im Zentrum der Strategie steht die industrielle Chemie, dem Trend zur Konzentration auf die Life Sciences folgt die BASF nicht.

\footnotetext{
115 „Verbund ist Geschichte und Zukunft. Als Friedrich Engelhorn 1865 die BASF gründete, hatte er eine Vision: Er wollte die Forschung für und die Herstellung von Textilfarbstoffen in einem Unternehmen integrieren. Die einzelnen Produktionen sollten aufeinander aufbauen, die Produkte und Reststoffe der einen Fabrik als Rohstoffe in der nächsten Anlage dienen. Heute ist das BASF-Werk Ludwigshafen der größte zusammenhängende Chemiekomplex der Welt. Alle 350 Betriebe sind mindestens über ein Produkt oder eine Prozessstufe mit anderen Betrieben verbunden. Diese effiziente Verknüpfung mit zahlreichen ökonomischen und ökologischen Vorteilen nennen wir Verbund. Doch der Verbund ist heute weit mehr. Verbund ist eine Philosophie. Der Verbund ist heute Teil unserer Unternehmensphilosophie und prägt viele Aktivitäten, sowohl nach innen, wie beim Know-howVerbund, als auch nach außen: im Verbund mit den Kunden, mit strategischen Partnern und nicht zuletzt mit der Gesellschaft. Er bündelt unsere Stärken für den globalen Erfolg und schafft dadurch Werte, von denen alle profitieren: unsere Partner und Kunden, das Unternehmen selbst und nicht zuletzt Gesellschaft und Umwelt. Prof. Dr. Jürgen Strube, Vorstandsvorsitzender der BASF Aktiengesellschaft, sieht "in der weltweiten konsequenten Umsetzung des Verbundes eine der wichtigsten Stärken unseres Unternehmens". Verbund ist ein Prozess. Unser Verbund ist einzigartig. Ein Prozess, den wir ständig optimieren und weltweit als Erfolgskonzept der BASF einsetzen: in Amerika, Europa und Asien. Ein Prozess ist flexibel, so auch der Verbund: Rund 100000 Mitarbeiter weltweit prägen und gestalten diesen Verbund.“

${ }^{116}$ http://www.basf.de/basf/html/rampe/home_d.htm, abgerufen im August 2002

${ }^{117}$ Als größtes Projekt gilt derzeit die BASF YPC Company Limited, ein Joint-Venture mit der chinesischen Sinopec, die eine 2,6 Milliarden Dollar teure Verbundanlage im ostchinesischen Nanjing bauen und betreiben will. Die Vorbereitungen zum Bau haben im Januar 2001 begonnen, die Produktion wird voraussichtlich Ende 2004, Anfang 2005 starten. Die Ethylen-Anlage wird eine Jahreskapazität von 600000 Tonnen haben, etwa 1,7 Millionen Tonnen chemische Produkte werden dann in dem Komplex verarbeitet. (Handelsblatt Nr. 239 vom 11.12.2000, Seite 19: „BASF gründet Joint Venture für Nanjing-Projekt“.)

${ }^{118}$ Die Trennung von Chemie- und Pharma-Aktivitäten ist Ende 2000 in vollem Gang und die BASF bereitet den Ausstieg aus dem Arzneimittelgeschäft vor. (Handelsblatt Nr. 243 vom 15./16. 12. 2000).
} 
Der Fall BASF muss vor dem Hintergrund der Entwicklung des Konzerns nach 1945 gesehen werden. Bei der Zergliederung der IG Farben hatte die BASF den größten und am stärksten integrierten Verbundchemiestandort der Welt erhalten, dessen Produktspektrum von der Industriechemie bestimmt war (Abelshauser 2002). Die Herstellung pharmazeutischer Erzeugnisse gehörte hingegen nie zum Kerngeschäft des Konzerns. Verstärkt wurde die Verbundzentrierung noch durch den Aufbau einer eigenen Energie- und Rohstoffbasis durch den Einstieg in das Öl- und Gasgeschäft. Globalisierung folgte viel eher noch dem Muster des Auf- und Ausbaus von Verbundstandorten für neue oder expandierende Märkte. Das bis heute dominante Organisationsprinzip ist das des fordistischen Großunternehmens in der spezifischen Form des deutschen Verbundchemieunternehmens. Der auf abgegrenzte Märkte bezogenen Produktionsorganisation entspricht eine hierarchischzentrale Unternehmenskoordination (Kädtler 2000).

\section{Hoechst - vom Chemie-Unternehmen zum Life Sciences Konzern}

Der Hoechst - Konzern geht seit Mitte der 1990er Jahre den Weg der Konzentration auf wenige erfolgreiche Kerngeschäfte. Die Konzernzentrale wird radikal verkleinert, der Vorstand beschränkt sich auf strategische Aufgaben, und operative Tätigkeiten werden den dezentralen Einheiten übertragen. Die Verselbständigung dieser Geschäftsbereiche reicht letztlich so weit, dass 1997 alle operativen Bereiche sowie die Servicefunktionen ausgegründet werden. Übrig bleibt eine „strategische Managementholding“ (Menz et al.: 106 ff) mit ca. 250 Beschäftigten, die den Hauptvorstand bei seinen Aufgaben unterstützen sollen. ${ }^{119}$ Der Vorstand steuert den Konzern über eine finanzielle Bewertung der Resultate, Entwicklungsmöglichkeiten und Ertragsperspektiven.

„Ziel ist, immer eine optimale Mischung aus neu aufzubauenden, wachsenden und reifenden Geschäftsfeldern zu haben. [...] An die Stelle der Optimierung von Wertschöpfungsketten soll - auf der Ebene der Konzernstrategie - das Renditepotential von ,Wertschöpfungsnetzwerken’ treten.“ (Dormann 1997)

Neben der höheren Wirtschaftlichkeit durch das Portfoliomanagement ist diese Akzentuierung für die Akteure bei Hoechst Voraussetzung für den Umbau des integrierten Chemie-Konzerns in einen Life Sciences Konzern, der sich zunächst auf die Arbeitsgebiete Pflanzenschutz, Ernährung, und Gesundheit auf der gemeinsamen Basis der Bio- und Gentechnologie stützt. 1999 schließlich erlischt der Name Hoechst, die AG geht in Aventis auf und verlagert ihren Firmensitz nach Straßburg. Diese Strategie ist gleichzeitig auch eine Absage an die Zentralforschung, diese wird in ein eigenständiges Unternehmen (Aventis Research \& Technologies GmbH und Co. KG) ausgegliedert.

Fünfzig Jahre nach seiner Gründung ist die aus Hoechst und Rhone Poulenc hervorgegangene Aventis kein deutsches Chemie-Unternehmen mehr mit Werken in vielen Ländern, sondern eine global agierende Unternehmensgruppe, die einen wesentlichen Teil ihrer Wurzeln in Deutschland und Frankreich und ihre Zentrale in Straßburg hat. Die zentrale Leitung des neuen Unternehmens erfolgt in den Bereichen Finanzen, Personal und Kommunikation und verbindet sich mit dezentraler, klar festgelegter operativer Verantwortung der rechtlich und in ihrem Auftreten eigenständigen Konzerngesellschaften, vor allem der Aventis Pharma in Frankfurt am Main und der Aventis Crop Sciences in

\footnotetext{
${ }^{119}$ Mit Gründung von Aventis, hat die Hoechst AG seit dem 15. Dezember 1999 eine neue Funktion. Sie fungiert nun als Zwischenholding, die als Beteiligungsgesellschaft der Aventis SA unter dem Aventis Logo firmiert. (http://www.hoechst.de/homepage/homepage.htm)
} 
Lyon. Aus dem integrierten chemisch-pharmazeutischen Unternehmen, welches noch nach der Verbundlogik funktionierte, ist so eine hoch spezialisierte und stark dezentralisierte Pharmagruppe geworden (Menz et al. 2000).

\section{Bayer-Modernisierung des chemisch-pharmazeutisches Konzepts}

Der Bayer-Konzern verfolgt demgegenüber eine moderatere Reorganisationsstrategie und ist bis heute ein breit diversifiziertes, integriertes Chemie- und Pharmaunternehmen. Bayer hält unter Verweis auf deren Aufgabe als „Kompetenzzentrum für Querschnittstechnologien und als Instanz der Personalentwicklung“ an der Zentralforschung fest (Kädtler 2000). ${ }^{120}$ Doch auch bei Bayer gewinnt der Ausbau der Life Sciences sowie der Landwirtschaft an Priorität, internes Wachstum hat aber den Vorrang vor Großakquisitionen ebenso wie vor Verkäufen größerer Sparten. ${ }^{121}$ Ausgründungen werden dann ins Auge gefasst, wenn die allgemeinen Konditionen wie Tarifbedingungen, interne Auflagen, Overheads etc. als nicht einlösbar angesehen werden. ${ }^{122}$ Die Konzernzentrale ist nach wie vor mit wesentlichen Entscheidungsbefugnissen ausgestattet, die zum Teil weit in die Geschäftsbereiche berührende Aktivitäten hinein reichen. Zwar ist der Vorstand seit Mitte der 1970er Jahre von der Ressortverantwortung enthoben, er behält aber regionale und funktionale Zuständigkeiten, die die individuelle Arbeitsteilung innerhalb des Gremiums abbilden. Dem Vorstand kommt damit in einer matrixartigen Entscheidungskonstellation als Gesamtgremium die Aufgabe zu, über die Vorlagen der Geschäftsbereiche zu entscheiden und dabei gleichzeitig als Querschnittsaspekt die funktionalen und regionalen Aspekte zu berücksichtigen. Die Organisation der Forschung und Entwicklung bleibt eine gemischte, die sowohl zentrale als auch dezentrale Elemente der Steuerung und Organisation beinhaltet und sich auf die Life Sciences - Sparten, Polymere, einige ausgewählte Grund- und Spezialchemikalien sowie auf Informationstechnologien konzentriert (Greb 2000).

Die Konzernzentrale bleibt einflussreichstes Gremium, und die strategische Richtlinienkompetenz des Vorstands reicht in wesentlichen Fragen in die Aktivitäten der Geschäftsbereiche hinein. Damit ist die Möglichkeit benannt, kurzfristig auf Kapazitäten anderer Geschäftsbereiche zurückzugreifen, die Vorteile gemeinsamer Landesorganisationen zu nutzen, einen langfristigen Risikoausgleich zu gewährleisten sowie an der Zentralforschung als Kompetenzzentrum für Querschnittstechnologien und Instanz der Personalentwicklung festzuhalten (Kädtler 2000). Obgleich die gesamte Geschäftsstrategie der einzelnen Sparten einschließlich der Investitions- und Standortpolitik in die Zuständigkeit der Geschäftsbereiche fällt, werden die marktorientierten Kalküle der Geschäftbereiche insofern relativiert, als der Vorstand im Einzelfall Entscheidungen auch gegen den Willen

\footnotetext{
${ }^{120}$ Dies gilt zumindest für den für unsere Zwecke relevanten Zeitraum bis 1999. Im Rahmen einer erneuten Restrukturierung der Unternehmensorganisation wird im Jahr 2000 die Zentralforschung aufgelöst

121 „Unseren Kunden bieten wir ein breites Sortiment von Produkten und Leistungen, das von den Bereichen Gesundheit und Ernährung über Kunststoffe bis zu Spezialprodukten in der Chemie reicht. Bayer ist forschungsorientiert und setzt bei seinen Kernaktivitäten auf die Technologieführerschaft. Dabei ist es unser Ziel, den Unternehmenswert nachhaltig zu steigern und im Interesse der Aktionäre, der Mitarbeiterinnen und Mitarbeiter sowie der gesamten Gesellschaft in allen Ländern, in denen wir vertreten sind, eine hohe Wertschöpfung zu erwirtschaften.“ (http://bayer.de).

${ }^{122}$ Das Outsourcing der Geschäftsbereichs Faser und Textilfarben kann insofern als Einzelphänomen bewertet werden.
} 
der Geschäftsbereiche durchsetzen kann - beispielsweise mit Blick auf Ressourcenallokation und Synergieeffekte.

Nehmen wir Bayer und Hoechst/Aventis als die beiden Pole strategischen Managements, so bilden im Fall Bayer langfristig gewachsene Beziehungen zwischen wissenschaftlichem Innovationspotential, industriellem Produktions- und Verfahrens - Know - How und Marktposition die Basis, die durch den Einsatz marktförmiger Controllinginstrumente effizienter ausgeschöpft werden. Im Gegensatz dazu setzt Hoechst/Aventis auf die Herauslösung von Kapital und Innovation aus dieser langfristigen Verbindung. Produktion und Innovation sollen durch finanzielle Impulse gesteuert werden.

Mit dieser Darstellung haben wir die Entwicklung in den 1990er Jahren grob erfasst. Dass Teile dieser Strategien inzwischen aufgegeben wurden bzw. inzwischen andere Schwerpunkte gesetzt werden, zeigt die Restrukturierung des Bayer-Konzerns im Jahr 2000, dem u.a. die Zentralforschung, die noch Mitte der 1990er als wichtiges Kompetenzzentrum galt, aufgelöst wird. Darüber hinaus verdichten sich Hinweise darauf, dass zumindest die Life-Sciences im Hinblick auf ihre kurzfristigen Synergie-Effekte überschätzt wurden. Unternehmen entscheiden sich zunehmend entweder für Pharma oder für „den Rest“, sprich Spezialitätenchemie etc.

Allen Akteuren gemeinsam ist aber bei aller Unterschiedlichkeit der daraus resultierenden Maßnahmen der Versuch, eine möglichst ausgewogene Orientierung an kurz- und langfristigen Wirtschaftlichkeitskriterien zu erreichen. So kommt Kädtler in seiner Analyse zu dem Schluss, dass die drei Fälle für Alternativen hinsichtlich der stofflich-funktionalen bzw. marktorientiertfinanziellen Aspekte der Unternehmenskoordination stehen, diese aber nicht auf objektive Rahmenbedingungen zurückzuführen sind. Es überwiegen differente strategische Optionen des Managements, die sich auf die Gegebenheiten des Unternehmens beziehen, die aber in hohem $\mathrm{Maß}$ von den persönlichen Einschätzungen und Interessen, Konsens- und Koalitionsbildungen wie Machtkonstellationen im Kreis der Entscheidungsträger geprägt sind. Damit ist eine anderen Branchen durchaus vergleichbare Situation umrissen, denn auch für die Unternehmen der Chemischen Industrie hat sich die Orientierung am Shareholder-Value-Konzept als wirkungsmächtiges Strategieprinzip durchsetzen können. Allerdings weist Becker in seiner Untersuchung zu Einfluss und Grenzen des Shareholder Value zu recht darauf hin, dass es durchaus fraglich ist, inwieweit es dem Management in Anbetracht der stofflichen Produktion überhaupt möglich ist, die Performance des Unternehmens stets an den Präferenzen und Erwartungen der Investoren und Kapitalmarktakteure auszurichten (Becker 2001). So führt einerseits die Umwandlung der Geschäftsbereiche in Profit Center nicht selten zu Dysfunktionalitäten der vorhandenen Verbundstrukturen. Andererseits stellt gerade die Wissenschaftsbasierung der Chemischen Industrie eine Variable im Rahmen der Reorganisation des industriellen Innovationsprozesses dar, die, wie im folgenden zu zeigen sein wird, erhebliche Konsequenzen für die Wandlungsprozesse hat. Hierin wird zugleich deutlich, dass die Analyse der Entwicklung des Produktionsstruktur allein nicht hinreichend ist, die Restrukturierungsdynamik der 1990er Jahre zu begründen. Denn sowohl der gesellschaftliche Transformationsprozess von der Industrie- zur Wissensgesellschaft wie auch der Paradigmenwechsel in der chemisch-pharmazeutischen FuE spielen eine zentrale Rolle und verstärken sich gegenseitig. Um neues Wissen zu entdecken und dann in einem bestimmten organisatorischen institutionellen Setting für spezifische Ziele anzuwenden, gilt es, neben organisatorischen Innovationen ein verändertes Forschungspotential zu etablieren und zu nutzen. 


\subsection{Das Innovationsmodell der Chemischen Industrie vor neuen technologischen Herausforderungen}

Aus heutiger Perspektive ist relativ nüchtern zu konstatieren, dass die seit den 1970er Jahren sich stetig beschleunigende Tendenz der Verbindung neuen Wissens zu neuen Technologien und wiederum zu neuen, qualitativ anderen technologischen Systemen eine entscheidende Basis für den Prozess sozioökonomischer Restrukturierung in den 1990er Jahren ist (Castells 2001). Der gesellschaftliche Bedeutungs- und Formwandel von Information und Wissen trägt damit, soviel kann nach über drei Jahrzehnten Debatte ${ }^{123}$ wohl mit einiger Sicherheit gesagt werden, auch zu einer Transformation der Industriegesellschaft hin zu einer „Wissensgesellschaft“ bei. ${ }^{124}$ Ökonomisch betrachtet gelten Wissen und Information heute als diejenigen Produktionsfaktoren und durchgängige Ressourcen, ${ }^{125}$ ohne die der „Motor des modernen Kapitalismus“ (Stehr 2001:15) ins Stocken gerät. Der Zusammenhang zwischen Information, Wissen und Innovation ist evident. Drei Dimensionen ${ }^{126}$ der Wissensgesellschaft sind insofern im Zusammenhang dieser Arbeit von Interesse, als sie eine erhebliche Modifikation der Entscheidungs- und Handlungskorridore (Ortmann 1995:39) für unternehmerische Strategien und Strukturen darstellen.

Erstens verändert sich mit den neuen Informations- und Kommunikationstechnologien die technische Infrastruktur der Unternehmen: Technik ist nicht mehr nur Produktionstechnik, sondern sie wird zur Unterstützung organisatorischer Informations- und Kommunikationsbeziehungen eingesetzt (Heidenreich 1998b). Im Gegensatz zur industriellen Revolution des 19. Jahrhunderts zeichnet sich diese aktuelle Entwicklung durch einen spezifischen Doppelcharakter aus. Denn die neuen Technologien sind nicht nur technische Artefakte, die als Hilfsmittel benutzt werden können, sondern darüber hinaus auch entwicklungsfähige Prozesse (Castells 2001). „Das Charakteristische der gegenwärtigen technologischen Revolution ist nicht die zentrale Bedeutung von Wissen und Information, sondern die Anwendung dieses Wissens und dieser Information zur Erzeugung neuen Wissens und zur Entwicklung von Geräten zur Kommunikationsverarbeitung und zur Kommunikation, wobei es zu einer kumulativen Rückkopplungsspirale zwischen einer Innovation und ihrem Einsatz kommt.“ (Castells 2001:34; Hervorhebung K.B.) Dieser Befund schließt die Existenz von Rückkopplungsmechanismen zwischen einzelnen gesellschaftlichen Teilbereichen innerhalb der Wissenschafts- und Technikentwicklung mit ein. Dies ist zunächst kein neuer Befund. Auch für die in der zweiten Hälfte des 19. Jahrhunderts

\footnotetext{
${ }^{123}$ Als einer der ersten brachte der us-amerikanische Managementtheoretiker Peter F. Drucker 1959 den Begriff des „Wissensarbeiters“ ein, Robert Lane prägte wahrscheinlich den Begriff der Wissensgesellschaft (1966), und Daniel Bell schließlich kennzeichnete die nachindustrielle Gesellschaft durch die zentrale Stellung theoretischen Wissens und die wachsende Wissenschaftsabhängigkeit technologischen Wandels (Bell 1973).

${ }^{124}$ Ich schließe mich damit dem derzeit gängigen Begriff an. Ob die neue Gesellschaftsformation als Informationsgesellschaft (Nora, Minc 1977), postindustrielle Gesellschaft (Bell 1973), Risikogesellschaft (Beck 1986), Netzwerkgesellschaft (Castells 1996) oder schlicht Wissensgesellschaft (Willke 1999) an die Stelle der Industriegesellschaft tritt, muss an dieser Stelle nicht diskutiert werden. Eine kritische Auseinandersetzung mit den unterschiedlichen Begriffen liefert Stehr (2001).

${ }^{125}$ Mit dem Begriff der Durchgängigkeit wird der Tatsache Rechnung getragen, dass die innovativen Technologien in alle Bereiche der Gesellschaft diffundieren und nicht etwa nur auf Teilsysteme wie die Wirtschaft oder die Wissenschaft beschränkt bleiben.

${ }^{126}$ Im folgenden steht nicht die umfassende Darstellung und kritische Erörterung der Debatte um die Wissensgesellschaft im Mittelpunkt. Vielmehr sollen die Aspekte und Dimensionen herausgearbeitet werden, die bezogen auf die Chemische Industrie relevant sind.
} 
entstehenden modernen naturwissenschaftlichen Disziplinen, ihre Autonomisierung und ihre Institutionalisierung im Hochschulsystem haben wir das enge Zusammenwirken von Akteuren chemischer Wissenschaft in den Universitäten und in den Forschungslaboratorien der Chemieindustrie nachweisen können (vgl. Kap. 2.2). Die Synergieeffekte zwischen Verfahrenstechnik und Chemischer Wissenschaft stehen ebenso für solche Rückkopplungen wie die Relevanz von Dampfmaschine und Elektrizität für den Take-off der Chemischen Industrie. Wissen und Information wurden also auch im Rahmen dieser Revolution transferiert und weiterentwickelt, so dass von Rückkopplungen zwischen den unterschiedlichen Sphären der Anwendung neuer Technologien durchaus gesprochen werden kann. Technologische Artefakte zur Informationsverarbeitung und zum Wissenstransfer wurden aber nicht gleichsam zum Kern der technologischen Transformation, sondern entwickelten sich parallel dazu.

Seit den 1970er Jahren jedoch zeichnet sich eine Konstellation ab, in der mittels einer gemeinsamen - digitalen - Sprache die Schnittstelle zwischen den unterschiedlichen wissenschaftlich-technologischen Bereichen /bspw. der Elektronik und der Biologie) überwunden werden kann. Dieses „Being Digital“ (Negroponte 1995) forciert nicht nur die technologischen Transformationsprozesse exponentiell, sondern legt auch den Grundstein für potentiell „grenzenlose“, das heißt unabhängig von Raum, Zeit und Fachdisziplin stattfindende Kommunikation und Informationsaustausch, die im Prozess der Nutzung innovativ weiterentwickelt werden können. Vom Anwender zum Innovator ist der Weg nicht weit, denn anders als die bisher bekannten Technologien sind viele heutige Innovationen nicht mehr unwiderruflich und eindeutig in ihrer Bedeutung fixiert wie etwa ein komplexes Rohrleitungssystem in der Chemieindustrie. Computer und Computerprogramme unterliegen einer ständigen Weiterentwicklung, sie sind Gegenstand eines permanenten Lern-, Forschungs- und Entwicklungsprozesses. Die in technischen Objekten verkörperten Erwartungen sind damit nicht mehr davor sicher, in Frage gestellt und revidiert zu werden (Knorr-Cetina 2002). Harte technische Fakten werden aufgeweicht, sie werden zu enttäuschungsbereiten Erwartungsmustern (Luhmann 1990). Eine experimentelle Haltung auch zu technischen Artefakten setzt sich durch, Technik ist zunehmend experimentell. $^{127}$

Die Rolle der Wissenschaft als vorrangiger Ort der Wissensproduktion verliert in diesem Zusammenhang nach Auffassung einiger Autoren an Bedeutung, da andere gesellschaftliche Teilsysteme (Kultur, Rechts, Wirtschafts oder Gesundheitssystem) zunehmend wissensbasiert seien. Mit der Ausdifferenzierung und Spezialisierung entwickeln sich darüber hinaus neue Formen der Produktion von Wissen, die auf inter- oder transdisziplinären Modi basieren, projektförmig organisiert sind und durch ihre auf Problemlösung zielende Ansätze einen hohen Anwendungsbezug aufweisen. Mitte der 90er Jahre stellten Gibbons et al. das Konzept eines „neuen Modus der Wissensproduktion“ zur Diskussion (Gibbons et al. 1994; Nowotny 1999). Diese - von den Autoren als „Modus 2“ bezeichne-

\footnotetext{
${ }^{127}$ Interessant ist eine historische Parallel, auf die Burke im Zusammenhang mit dem von ihm als ,pragmatischen Skeptizismus“ bezeichneten Phänomen hinweist. Er umschreibt damit die grundsätzlich misstrauischen Haltung gegenüber Wissensansprüchen, die sich im 17. und 18. Jahrhundert durchsetzte und zu einer „Krise des Wissens im Europa des späten 17. Jahrhunderts“ (Burke 2001:237) führte und letztlich den Weg für den Aufstieg der Naturwissenschaften als „wahrer“, da empirisch-methodisch zu fundierender Wissenschaft. Ob und inwieweit die derzeitige Situation eine neue „Krise des Wissens“ darstellt, soll hier nur angedeutet werden. Insgesamt scheint aber der postmoderne Begriff des Wissens geeignet, eine solche Krise zu diagnostizieren (Lyotard 1999).
} 
te - Art der Wissensproduktion sei neben die traditionelle, institutionell vor allem in der akademischen Forschung und Wissenschaft beheimatete Form gesellschaftlicher Wissensproduktion („Modus 1“) getreten. Jenseits der durchaus kontrovers diskutierten Frage, ob es sich tatsächlich um einen neuen Modus handelt (Weingart 2001; Hack 2001), und inwieweit es zu einer Verwissenschaftlichung der Gesellschaft oder aber einer Auflösung der Wissenschaft in die Gesellschaft kommt (Willke 2001), sind die Befunde bezogen auf das Verhältnis von Wissenschaft und Gesellschaft recht eindeutig: Die traditionell herausgehobene gesellschaftliche Position der Wissenschaft erodiert. Neues wissenschaftliches Wissen wird nach diesem Verständnis immer weniger allein innerhalb des herkömmlichen Wissenschaftssystems, sondern in vielen autoorganisierenden Räumen und zunehmend heterogeneren Formen der institutionellen, organisationalen und disziplinären Ordnung erzeugt.

Ein zweites zentrales Element der Wissensgesellschaft folgt aus dem spezifischen Charakter von Wissen und hat weitreichende Folgen für „Wissen“ als Produktionsfaktor. Denn im Gegensatz zu anderen materiellen Produktionsfaktoren ist Wissen nicht im herkömmlichen Sinn zu akkumulieren, es ist nicht von einer linearen Ansammlung von Wissen auszugehen oder von „addierbaren Wissensquanten“ (Scharping 2000:47). „Wissen ist keine zuverlässige Ware, es ist zerbrechlich und stellt eigene Anforderungen. Es ist mit Unsicherheit verbunden.“ (Stehr 2001:116) Wissen ist immer eingebunden und wird auf anderes Wissen, andere Erfahrungen, Urteile etc. bezogen. Es ist eher als Gewebe unterschiedlicher Praktiken zu verstehen und kann damit je nach Kontext einen unterschiedlichen Mehrwert haben: Lehrbuchwissen steht Erfahrungswissen gegenüber, implizites Wissen dem expliziten Wissen und emotionales Erleben wird gegen lebensweltliches Hintergrundwissen ausgespielt. Wissen ist durch diese spezifischen phänomenologischen Eigenschaften schwer kodifizierbar, und für Unternehmen etwa stellt sich dadurch schon seit langem die Frage, wie sie wissen können, was sie wissen (Lullies et al. 1993). Unterschiedliche Akteursgruppen haben darüber hinaus in Unternehmen zumeist deutlich voneinander abweichende Wissensbestände, die sie zusätzlich je nach den geltenden sprachlichen Vereinbarungen in der Disziplin, der Berufsgruppe bzw. der community of practice (Brown, Duguid 1991) je unterschiedlich festgelegt haben. Dieser sozial-kommunikative Kontext ist es auch, der darüber entscheidet, ob Wissen überhaupt verwertet werden kann. Wissen verstanden als wissenschaftliche Erkenntnis und Technik erzwingt also nicht automatisch seine praktische Realisierung. Obgleich Wissen die Befähigung zum Handeln impliziert, handelt es sich nicht um konstante und kontextunabhängige Chancen (Stehr 2001). Mehr Wissen bedeutet darüber hinaus nicht mehr Gewissheit zu besitzen. Die Wissensgesellschaft ist gerade nicht durch eine ständige Erweiterung des Wissens und eine parallel dazu verlaufende Abnahme des Nichtwissens gekennzeichnet. Krohn (1997) verweist darauf, dass neues Wissen immer mit neuen Ungewissheiten, Unsicherheiten, Risiken und Widersprüchlichkeiten einhergeht. Diese Erkenntnis ist als solche nicht neu, sondern kennzeichnend für moderne Gesellschaften. Erst in der Wissensgesellschaft aber, so Krohn, wird dieser ambivalente Charakter von Wissen handlungsleitend und die Wissensgesellschaft „,eine Gesellschaft, die in ständig wachsendem Maß über den Umfang und die Ebenen ihres Nichtwissens lernt“ (Krohn 1997:69). „Wissensgesellschaft würde dann eine Gesellschaft bezeichnen, die ihre Existenz auf solche experimentellen Praktiken gründet, die unvorhersagbar in ihrem Ausgang und unbekannt in ihren Nebenfolgen sind und daher ständiger Beobachtung, Auswertung und Justierung bedürfen. Die Wissensgesellschaft ist eine Gesellschaft der Selbst-Experimentation.“ (ebd. 70). Es ist, im An- 
schluss an Heidenreich, eben diese Bereitschaft zur stärkeren Infragestellung eingelebter Wahrnehmungs- und Handlungsmuster, zur Veränderung der eigenen „Realitätsgewissheiten“, die qualitativ neu im Umgang mit Wissen ist. Normative Stilisierungen von Erwartungen treten immer seltener mit dem Anspruch auf überzeitliche und überörtliche Gültigkeit an, sondern werden als prinzipiell veränderbar verortet. „Die Regeln und Selbstverständlichkeiten der Gesellschaft werden häufiger in Frage gestellt - und dies dokumentiert sich in der beschleunigten Erosion bisheriger Regulationsstrukturen und in der Entwicklung neuer Regeln. Ein zentraler Indikator für eine stärkere Wissensbasierung ist also eine beschleunigte Oszillation zwischen Deregulierung und Neuregulierung“ (Heidenreich 1998:2; Hervorhebung im Original). Es sollte deutlich geworden sein, dass das Neue nicht in der Anerkennung eines wie immer quantitativ und qualitativ ausgeformten Nicht-Wissensbestands liegt, sondern das die aktuelle Situation geprägt ist von den Versuchen, die jeweiligen Formen des Nicht-Wissens in möglichst allen Phasen der Wissensproduktion und der Anwendung wissenschaftlicher Erkenntnis und Technik zu erfassen. Eine allgemeine Formulierung für das Phänomen des Nichtwissens ergibt sich aus den systemtheoretischen Überlegungen von Luhmann, der Wissen als Beobachtung fasst und daran anschließend schlussfolgert: „Jede Beobachtung bewirkt, dass die eine Seite einer Unterscheidung bezeichnet wird und die andere folglich unmarkiert bleibt" (1992:155). Daraus folgt, dass selbst wenn im weiteren Verlauf die andere Seite der Unterscheidung beobachtet würde, dies wieder eine weitere Seite unbeobachtet ließe und so fort. Es kann damit nicht zu einer Umwandlung von Wissen in Nicht-Wissen kommen, sondern Wissensakkumulation impliziert lediglich die „progressive Reproduktion von Nichtwissen“ (Luhmann 1992: 177). ${ }^{128}$ Deutlich wird, vor welcher Schwierigkeit die Wissensproduzenten und damit auch die Organisation der Innovationsprozesse stehen. Denn Nichtwissen steht nicht nur am Anfang oder am Ende einer technologischen Erprobung, sondern wird im Verlauf der Implementation erarbeitet und wenn möglich über rekursive Rückkopplungsspiralen wieder in den Prozess eingebracht. Denn: Die Auflösung des Nichtwissens in bearbeitbare Probleme und Lösungen ist stets verbunden mit der Erzeugung neuen Nichtwissens (Krohn 1997: 84). Willke betont von daher zu recht, dass eine entscheidende zusätzliche Voraussetzung der Wissensgesellschaft ist, Wissen und Expertise einem Prozess der kontinuierlichen Revision zu unterwerfen. ${ }^{129}$

Drittens schließlich verändern sich auch die Wissenschaftsdiskurse insbesondere in der Biologie und Medizin. ${ }^{130}$ Die Unterscheidung des Normalen vom Pathologischen bezogen auf den biologischen und medizinischen Körper basiert bis Mitte des 20. Jahrhunderts auf

\footnotetext{
${ }^{128}$ Wehling (2002) fasst darüber hinaus für den Innovationsprozess drei unterschiedliche Dimensionen des so entstehenden Nichtwissens zusammen: Erstens bezeichnet er mit explizit gewusstem und vollständig unerkanntem Nichtwissen die idealtypischen Pole des Wissens um das Nichtwissen. Zweitens unterscheidet Wehling Intentionalität des Nichtwissens mit den Extremen bewusst gewolltes Nichtwissen und gänzlich unbeabsichtigtes Nichtwissen. Drittens schließlich bezeichnen bloß temporäres Nichtwissen (Noch-Nicht-Wissen) und unauflösbares Nichtwissen (Nicht-Wissen-Können) die beiden Antipoden bezogen auf die zeitliche Stabilität und Dauerhaftigkeit.

${ }^{129}$ Obgleich der Bezug zu postmodernen Autoren bei Willke nicht auftaucht, geht die Charakterisierung von „Wissen in der Wissensgesellschaft“ doch in dieselbe Richtung, wie sie etwa Lyotard bereits 1979 wies: „Wie gesagt, der auffallende Zug des postmodernen Wissens besteht in der - jedoch expliziten - Immanenz des Diskurses über die Regeln, die seine Gültigkeit ausmachen“ (1999:159).

${ }^{130}$ Diese Veränderung bedeutet nicht, auch wenn im folgenden einige Stilisierungen und Polarisierungen vorzunehmen sind, dass ein Diskurs durch einen anderen ersetzt wird. Vielmehr ist davon auszugehen, dass sich die diskursiven Praktiken sowohl ergänzen und überschneiden als auch parallel nebeneinander stattfinden.
} 
einem hierarchisch-funktional und arbeitsteilig organisierten System (Haraway 1995). Dieser Vorstellung liegt die Annahme zugrunde, dass alle Lebensvorgänge in chemischen Kategorien beschreibbar sind. Diese aus den Anfängen der Arzneimittelforschung resultierende und sowohl theoretisch wie experimentell nachgewiesene Grundlage bildet den Ausgangspunkt des chemischen Paradigmas. FuE in der pharmazeutischen Industrie kaprizierte sich darauf, ,gestörte Fließgleichgewichte und Zusammensetzungen durch die Zufuhr definierter chemischer Stoffe zu normalisieren“ (Drews 1998:101). Nach dem Zweiten Weltkrieg schließlich wandelt sich das wissenschaftliche Sprechen über Natur, Körper und Krankheit tiefgreifendend. 1944 wird die chemische Basis der Gene entschlüsselt, und der damit einsetzende Paradigmenwechsel in den Naturwissenschaften findet seinen symbolischen Ausdruck 1953 in der Darstellung der Struktur des genetischen Codes in Form einer Doppelhelix durch Crick und Watson. Die DNA-Polymerase wird als für die Verdoppelung der Helix zuständige Substanz isoliert und charakterisiert und grundlegende Vorgänge der Übersetzung von DNA in RNA (Transkription) und der Übersetzung der Ribonukleinsäuren in Proteine (Translation) beschrieben. 1972 schließlich gelingt es, den DNA-Faden gezielt in einzelne Gene zu zerlegen, sie zu isolieren und ihre Funktion zu analysieren. Mit dem Wissen um die Vorgänge in den Zellen auf molekularer Ebene sowie den innovativen Verfahren zur Identifikation, Isolierung und Neukombination genetischer Informationen ist es fortan möglich, Artgrenzen zu überschreiten, indem Gene isoliert und in andersartige Lebewesen eingebunden werden

Die sich aus diesen Pionierarbeiten sukzessive entwickelnde molekulare Genetik wird als die bisher umfassendste Möglichkeit angesehen, Krankheiten zu verstehen, zu diagnostizieren, und zu behandeln. Insofern hat sich ein informationelles Paradigma durchsetzen können, als dessen „Generalschlüssel“ (Drews 1998:111) die Molekularbiologie gilt und in dem Krankheiten als Informationsdefizite oder überschüsse definiert werden. Diese zunächst nur in ihrer Relevanz für die pharmazeutische Forschung beschriebene biomedizinische Entwicklung korrespondiert eine nicht von dieser zu trennende biotechnologische, ${ }^{131}$ die neben bzw. in Verbindung mit Technologieentwicklungen im Bereich der Informations- und Kommunikationstechnologien als Triebkraft gesellschaftlicher Wandlungsprozesse gelten kann. Die Biotechnologie kennzeichnet ein Gebiet wissenschaftlicher und technischer Entwicklungen, ${ }^{132}$ und definiert sich als integrierte Anwendung unterschiedlicher Disziplinen für die Nutzung der Stoffwechselprozesse von Organismen, Zellen oder Teilen davon zur Herstellung von Gütern und Dienstleistungen (Bull et al. 1982). Biotechnologie steht dabei einmal quer zu den etablierten Disziplinen und forciert interdisziplinäres Zusammenwirken (etwa im Bereich der Bioinformatik oder der Pharmacogenomics). Sie betrifft darüber hinaus in ihren potentiellen Einsatzgebieten als Querschnittstechnologie nicht nur naheliegende Bereiche wie Medizin, Landwirtschaft und Ernährung, sondern auch Umweltschutz, Energie- und Rohstoffgewinnung. ${ }^{133}$ Ihren

\footnotetext{
${ }^{131}$ Für die folgenden Ausführungen gilt, dass gemäß dem inzwischen durchgesetzten Sprachgebrauch durchgängig von Biotechnologie die Rede sein wird, obgleich zu trennen wäre zwischen a) der traditionellen Biotechnologie, die etwa bei der Hefegärung im Spiel ist und zur Herstellung von Produkten wie etwa Bier, Brot und Käse , b) der modernen Biotechnologie, die industrielle Fermentationstechniken umfasst sowie c) der Gentechnologie, die es ermöglicht, Gene zu isolieren und zu übertragen.

${ }^{132} \mathrm{Zu}$ nennen sind hier die Molekular, Mikro und Zellbiologie, die Biochemie, die Immunologie, die Virologie sowie auch die Umwelt- und Verfahrens- wie Informations- und Kommunikationstechnologien.

${ }^{133}$ Von besonderem Interesse ist die Biotechnologie etwa bei der Herstellung von Feinchemikalien. Entscheidende Triebkraft sind die wesentlich geringeren Produktionskosten im Rahmen biotechnologischer Verfahren (be-
} 
Ausdruck findet diese Komplexität in den Bezeichnungen rote (Arzneimittel, Impfstoffe, Medizinprodukte, Diagnostika), grüne (Pflanzenzüchtung, Nahrungsmittelherstellung), blaue (maritime Forschung) und graue (Umweltbiotechnologie) Biotechnologie. ${ }^{134}$

\subsection{Neue betriebliche Formen der Organisation von Innovation}

Resümiert man diese Befunde, so deutet sich bereits an, dass diese Entwicklungen für die wissenschaftsbasierte Chemische Industrie qualitativ neue Anforderungen an das Innovationsmodell beinhalten. Im Innovationsprozess wird neben den von Schumpeter (1987) benannten Konkurrenzen um neue Ware, neue Technik, neue Versorgungsquellen und neue Organisationstypen die Konkurrenz um neues Wissen immer relevanter. Zwar weist die Branche, dies zeigte Kapitel 2, von jeher eine enge Kopplung an die wissenschaftliche Forschung auf und betreibt eigene grundlagenorientierte Forschung. Wissen wird hier traditionell im Kontext bestimmter Infrastrukturen in oft komplizierten, umfassenden sozialen Netzwerken erzeugt, verfügbar gemacht und verbreitet, interpretiert und mit lokalen Gegebenheiten verbunden, bevor es implementiert oder realisiert wird. Die Herstellung und die Übersetzung von Wissen in technische Artefakte ist ein komplexer, wandelbarer intellektueller wie auch organisatorischer Prozess, in dessen Verlauf die Chemieunternehmen sowohl auf interne wie auf externe Wissensbestände rekurrieren. Scheitern und Rückschläge gehören zur Forschungs-Normalität. Prognostizieren und exakte Planung von Innovationen sind - wie im übrigen in allen Branchen und jede Innovation - schwierig bis unmöglich (Stehr 2001; Latour 1993; Gibbons et al. 1994). Fest steht aber, dass sich mit dem Wissenschafts- und Technologieschub für den Bereich der industriellen Anwendung ein Innovationspotential eröffnet, dessen Realisierung und Ausschöpfung folgenreich für die strategische wie strukturelle Ausrichtung der Unternehmen ist. Nicht nur Markt- und Wettbewerbsstrategien sind im Hinblick auf die nun potentiell zu generierenden Produkte neu zu ordnen, auch weitreichende organisatorische Innovationen im Bereich der Forschung und Entwicklung sind durch die veränderten Rahmenbedingungen der Wissensproduktion zu bewältigen (Briken, Kurz 2003): Wissen basiert neben den etablierten (chemischen) Forschungsmethoden und ansätzen auf einer neuen (biotechnologischen) Grundlage, die durch eine „Hegemonie der Information“ (Castells 2001) gekennzeichnet ist. ${ }^{135}$

Dies wird einmal deutlich an einem Bedeutungszuwachs externer FuE. Mit der Anzahl der Akteure und dem bedingt durch die Biotechnologie qualitativen wie quantitativen Wandel des Wissens steigt auch die Vergabe der FuE-Aufträge nach außen. Dies gilt zwar vor allem für die Pharmasparten, hier werden derzeit etwa 30 Prozent der primären

\footnotetext{
dingt etwa durch im Vergleich zu chemischen Produktionsverfahren wesentlich niedrigere Drücke, gemäßigte Temperaturen sowie milde pH-Werte) (vgl. Festel et al. 2004).

${ }^{134}$ Diese Definitionen sind insofern irreführend, als sie den hybriden Charakter der Biotechnologie nicht widerspiegeln können. So bleibt etwa unklar, ob Pflanzen, die mit Vitaminen oder pharmazeutischen Wirkstoffen angereichert werden, noch als Nahrungsmittel, also grüne oder bereits als Arzneimittel, rote Biotechnologie gelten sollen.

${ }^{135}$ Die folgenden Ausführungen basieren neben eigener Literaturrecherche maßgeblich auf Ergebnissen des Forschungsprojekts „Die Organisation von Innovationen in forschungsintensiven Branchen am Beispiel der Biotechnologie“ (Projektleitung V. Wittke, Bearbeitung: C. Kurz, K.Briken) sowie auf intensiven Diskussionen mit C. Kurz , für deren produktiven Anregungen und konstruktive Kritik an dieser Stelle nochmals gedankt sei.
} 
Forschungskosten in Kooperationen investiert. Vor allem der Rückgriff auf die WissensRessourcen der Biotechunternehmen wird in diesem Bereich genutzt, um die unternehmenseigenen Innovationspotentiale gezielt zu stärken. Für die Chemische Industrie insgesamt gilt, dass der Anteil der externen FuE-Aufträge an den gesamten FuEAufwendungen zwischen 1995 und 1997 von knapp drei auf sechs Prozent angestiegen ist (ZEW 2003:29), ${ }^{136}$ und im Branchenvergleich werden überdurchschnittlich oft Aufträge an Hochschulen vergeben. Darüber hinaus hat die FuE-Arbeitsteilung auf internationaler Ebene zugenommen. Und so halten die deutschen Chemieunternehmen derzeit etwa 40 Prozent ihrer inländischen FuE-Kapazitäten im Ausland. An dieser Entwicklung zeigt sich deutlich der ambivalente Charakter des neuen Wissens. So machen es zwar einerseits die neuen Technologien möglich, Wissenstransfer und Kooperation über weite Distanzen ohne zeitliche Verluste zu betreiben. Allerdings ist die Übertragung von Wissen ein ,aktiver Prozess und nicht der Prozess kommunizierender Röhren“ (Stehr 2001:52), und gerade im Forschungsbereich ist die Einbindung in die lokalen Wissensgemeinschaften und communities of practice unter den veränderten Unsicherheitsbedingungen deutlich relevanter geworden. Standortbedingungen werden unter diesen Voraussetzungen wieder relativ unflexibel. „Wer etwa heute in der forschenden Pharmaindustrie in eine neue Indikation einsteigen will, muss Forschungskapazitäten oft dort ansiedeln, wo sich die auf diesem Gebiet führende scientific community herausgebildet hat, weil Partizipation an der Spitze der Entwicklung allein über das Herauskaufen selbst von Spitzenleuten oft nicht möglich ist" (Kädtler 1999:23) Für die Chemische Industrie und ihre Globalisierungstendenzen auf geographischer Ebene ist denn auch festzustellen, dass die globalen Netze der Unternehmen einmal durch die Verlagerung von Produktionsstätten im Bereich der forschungsextensiven Marktsegmente gekennzeichnet ist, andererseits aber durch die Konzentration qualifikationsintensiver, technologisch komplexer Bereiche und Funktionen, insbesondere auch FuE, an Standorten in den Zentren der Triade (USA, Japan, Europa) konturiert werden (Kädtler 2000; Koubek 1997; Dolata 1997).

Parallel zu dieser Reorganisation der Außenbeziehungen wird unternehmensintern Innovation als Querschnittsaufgabe definiert: Nach einer Studie der Unternehmensberatung Arthur D. Little betonen die Vertreter der befragten Chemie- und Pharmaunternehmen, dass Innovation nicht mehr nur in den Zuständigkeitsbereich von Forschung und Entwicklung fällt, sondern sie explizit als Teil der Unternehmensstrategie verstanden wird, d.h. „sie geht jeden etwas an“ (ADL 1998: 55). Bezogen auf die Organisation bedeutet dies, dass die funktionsbezogene Arbeitsteilung und die damit verbundenen Schnittstellen- und Koordinationsprobleme reduziert werden, indem Innovation als durchgängiger Prozess von der Forschung bis zum Marketing aufgebaut wird. Funktional ausdifferenzierte Teilsysteme interagieren, so dass frühere „Innovationsanwender“ zu Ko-Innovatoren werden können. Diese querfunktionale Kooperation ermöglicht den Einbezug aller für den Wertschöpfungsprozess relevanten Wissensbestände und findet seinen Ausdruck in einer zunehmend an Projekten orientierten Organisationsstruktur, meist in Form einer Matrixorganisation. Maßgeblich für den Innovationsprozess ist nun die simultane, d.h. gleichzeitige und überlappende Entwicklung von Produkten und Prozessen. Diese Form des „Busi-

\footnotetext{
${ }^{136}$ Diese Zahl ist bezogen auf die gesamte deutsche Industrie gering, denn hier liegt der Anteil bei ca. 15 Prozent. Dies steht jedoch nicht für eine besondere Zurückhaltung in der Chemischen Industrie, sondern belegt noch einmal eindrücklich, dass die Branche historisch gesehen auf hohen FuE-Aufwendungen basiert und eher noch als Innovationsgenerator für andere Branchen anzusehen ist (ZEW 2003).
} 
ness Reengineering“ im Rahmen einer prozessorientierten Organisation ist derzeit das neue Paradigma der Unternehmensgestaltung. Es stellt jedoch für die Chemieunternehmen eine doppelte Herausforderung dar. Die Unternehmen müssen einmal gewährleisten, dass die einzelnen Prozesse entlang der Wertschöpfungskette effektiv verknüpft werden. Dies allein ist keine leichte Aufgabe, denn schließlich sollen Akteure mit unterschiedlichen sozialen Kontexten, etwa aus dem Bereich der Entwicklung und des Marketing, fortan Wissen kommunizieren. Zusätzlich gilt es aber auch, den bereits skizzierten transund interdisziplinären Anforderungen der aktuellen Wissenschaftsentwicklung Rechnung zu tragen. Die Akteure aus dem Bereich der FuE müssen nun die anspruchsvolle Aufgabe lösen, nicht nur die Vernetzung vorhandener Wissensbestände und die Integration externer Expertise zu gewährleisten, sondern völlig neue Forschungslinien und disziplinäre Zusammenhänge etablieren (Drews 1998; Kurz 2002; Briken, Kurz 2003). Insgesamt ändert sich damit nicht nur der Gehalt von Wissen, sondern auch die Qualität der kommunikativen Vermittlungsleistungen. Die Innovationsleistungen hängen nun in besonderer Weise davon ab, wie das Problem der Auswertung dieses komplexen und heterogenen Wissens organisatorisch angegangen wird. Der Zustand eines Kommunikationsnetzwerkes entscheidet darüber, welches Wissen im Prozess der Innovation zum Zuge kommt und welches nicht (Baecker 1999:81f).

Zusammenfassend ist festzustellen, dass zwar Wissen in der Chemischen Industrie schon immer die Grundlage bzw. ein wesentlicher Anhaltspunkt für Entscheidungen im Innovationsprozess liefert. Der Wert von Wissen definiert sich von jeher nicht durch seine Existenz, sondern in hohem Maß darüber, was es im Prozess der Innovation in Gang setzen kann. Die Unternehmen versuchen nun bei ihren Restrukturierungsbemühungen, die optimalen Rahmenbedingungen für dieses „in Gang setzen“ zu definieren, um die Produktion, Verbreitung und Anwendung von Wissen zu forcieren. Das Zusammenwirken der unterschiedlichen wissenschaftlichen, ökonomischen und technologischen Entwicklungen in den Unternehmen stellt eine komplexe Gemengelage dar, die sich in drei Bedingungen bündeln lässt: Erstens bestehen mit der Wissenschafts- wie der Marktentwicklung zwei unterschiedliche Referenzsysteme mit je bestandskritischen Ansprüchen an die Unternehmen. Die Existenz der daraus resultierenden partiell widersprüchlichen Erwartungen erzeugt einen erheblichen Druck auf die beteiligten Akteure, und zwar auf allen Ebenen. Zweitens erfordert die Erfüllung der unternehmerischen Aufgaben eine hohe Kapazität zur Wissens- und Informationsverarbeitung, denn eine Fülle neuer Informationen und neuen Wissens dringt in die Firmen ein, und dies kann aufgrund seines Querschnittscharakters nicht mehr allein von einer Spezialistenstelle isoliert bearbeitet werden. Zusätzlich ist die Situation durch eine hohe Unsicherheit, Diversität und Interdependenz gekennzeichnet. Und schließlich verlangen drittens die Produkte und Projekte immer häufiger nach einer gemeinsamen Ressourcennutzung von Aggregaten, Spezialeinrichtungen und Wissensbeständen. Zu bedenken ist dabei nicht nur der Umgang mit den unterschiedlichen Wissensbeständen in Unternehmen, sondern auch der wissenschaftliche Paradigmenwechsel. Die organisatorische Aufgabe besteht darin, implizites in explizites Wissen zu modifizieren, mit neuem Wissen zu verbinden und diese „aktualisierten“ Bestände für organisatorische Entscheidungsprozesse zu nutzen (Nonaka, Takeuchi 1997; Baecker 2003).

Konventionelle organisatorische Lösungen wie etwa die Linien- oder Stab-LinienOrganisation sind für diese komplexe Problemlage zunehmend ungeeignet, da sie zumeist 
nur einem Aspekt eines Problembereichs auf den verschiedenen hierarchischen Ebenen in den Blick nehmen (können), und auch in den Chemieunternehmen kommen zunehmend Matrixstrukturen zum Einsatz. Die traditionelle, nach Funktionen gegliederte Organisationsstruktur (vertikale Linie) wird in der Matrix in der horizontalen von einer projekt- oder produktorientierten Struktur überlagert, so dass sich im Schnittpunkt je zwei Kompetenzsysteme, in der Regel Funktions- und Objektsystem kreuzen, also Verrichtungen wie Forschung, Entwicklung, Produktion und Produkt- bzw. Projektgruppen zueinander in Beziehung gesetzt werden. ${ }^{137}$ Die Weisungskompetenz und Verantwortung zwischen den Instanzen werden dann geteilt: Der Produkt- bzw. Projektmanager ist für Planung, Koordination, und Kontrolle der Projektarbeit (Zeitplan und Inhalte) zuständig, wobei er die höchste Priorität für das von ihm bearbeitete Projekt anstrebt und es im Verlauf über die einzelnen Funktionen hinweg verfolgt. Der Funktionsmanager hingegen koordiniert und bestimmt die Ressourcenbereitstellung (personelle wie auch finanzielle Ausstattung), wobei er alle Projekte im Blick hat und die Kapazitätsauslastungen beachtet (Staehle 1996). Das Spezifische der Matrix ist nun, dass bei Abstimmungen organisatorisch keine bestimmte dominante Lösung zugunsten des einen oder anderen Kompetenzsystems vorgesehen ist. Mit dieser Struktur, die auf der Überlagerung von Kompetenzsystemen basiert, ist also eine Situation geschaffen, der ein vergleichsweise hohes Konfliktpotential innewohnt. Konflikte werden allerdings in diesem Konzept nicht mehr als Bedrohung der organisatorische Ordnung verstanden, sondern als produktives Element, das Abstimmungsund Schnittstellenprobleme argumentativ zugänglich macht. Diese „Institutionalisierung des Konfliktes“ (Schreyögg 1993) wird von den Unternehmen als Instrument zur Bewältigung der komplexen externen wie internen Anforderungen genutzt, um „zu innovativen, den Wandlungsanforderungen gerecht werdenden Problemlösungen“ zu kommen. ${ }^{138}$ Lösungen der innerhalb von Matrixstrukturen entstehenden Probleme werden dann in der Regel nicht durch Anweisungen, sondern durch Verhandlungen und gegenseitige Abstimmungen erzielt. Dabei ist diese Form der Matrix nicht nur auf der makrostrukturellen Ebene sondern gerade in kleinere Subeinheiten als organisationale Verbindungseinrichtung wirkungsvoll. Festzustellen ist, dass Matrixstrukturen in den Teilbereichen des Unternehmens angewendet wird, die durch die simultane Berücksichtigung von zwei Dimensionen gekennzeichnet sind und unter hohem Innovationsdruck stehen. Denn, dies belegen einschlägige Untersuchungen (Kanter 1983), die der Matrixorganisation zugrunde liegende Mehrperspektivität und die daraus resultierende kritische Distanz zu langjährig entwickelten Einzel-Perspektiven unterstützt in besonderer Weise die Innovationsfähigkeit von Unternehmen (Schreyögg 1993). Dabei verlangt die Matrixstruktur sowohl eine Revision des traditionellen hierarchisch strukturierten Autoritätsgefüges wie auch eine Abkehr von eingeübten Verhaltens- und Denkweisen und stellt so veränderte Anforderungen an die beteiligten Akteure.

\footnotetext{
${ }^{137}$ Möglich sind neben dieser klassischen Polarisierung aber auch Kombinationen andere Dimensionen der Abteilungsbildung denkbar, geläufig ist etwa die nach Objekt und Region, in der sogenannte Tensor-Organisation werden sogar drei Aspekte (Verrichtung, Objekt, Region) miteinander verbunden (Schreyögg 1993).

${ }^{138}$ Marr, Stitzel 1979 zitiert nach Schanz 1995:110.
} 


\subsection{Neubewertung von Arbeitskraft}

Wir haben in Kap.1 bereits festgestellt, dass der Wandel der Unternehmen zur dezentralen, prozessorientierten Organisation weitreichende Implikationen für die Beschäftigten hat, horizontale Abstimmungsmechanismen an Bedeutung gegenüber vertikaler Anweisung gewinnen und der Bedarf an Kooperation und Kommunikation steigt. ${ }^{139}$ Die prozessorientierte Organisation basiert auf der Grundannahme, dass Innovationen vor allem entwickelt werden können, „weil Wissen geteilt und eben nicht monopolisiert wird“ (Stehr 2001). ${ }^{140}$ Dieses Konzept drückt sich in den Unternehmen in vielfältigen hierarchie- und fachübergreifenden Kooperationsformen aus, deren Ziel es ist, im Rahmen der existenten Funktions- und Arbeitsteilung neue „Kommunikations- und Wissensbrücken“ (Kurz 2002) aufzubauen. Mehr Akteure und heterogenere Leistungen sind ins Spiel gekommen, und als zentrales Medium zur Steuerung und Umsetzung der Innovationsprozesse haben sich Projektgruppen entwickelt. Die Unternehmen passen ihr bürokratischhierarchisch und funktions- wie arbeitsteilig geprägtes Organisationsmodell an die Erfordernisse der prozessorientierten Organisation an, indem sie „Querfunktionalität“ auf gleicher Ebene etablieren. Bereits Kern und Sabel (1994) hatten dies zu einer wesentlichen Voraussetzung für Produktivitätsfortschritte und Innovationsbeschleunigung erklärt, und Projektgruppen gelten derzeit als Medium, diese Anforderung organisatorisch und verhaltenspraktisch zu gewährleisten.

Der Organisationskontext von Produkt- und Technikentwicklung verändert sich damit erheblich und beinhaltet neue Anforderungen, Chancen und Risiken für die Beschäftigten in Forschung, Entwicklung und Produktion. Diese interne Reorganisation betrifft Status, Kompetenz und Arbeitssituation aller Arbeitskräfte, allerdings, dies zeigen aktuelle Befunde, verläuft die Entwicklung für einzelne Bereiche nicht einheitlich, und ,je nach Tätigkeitstyp, Fachabteilung und hierarchischer Ebene differieren Gehalt und Komplexität der Innovationsaufgabe sowie die Möglichkeiten zur Beteiligung an Innovationsaktivitäten“ (Kurz 2002:601). Wir wollen im folgenden zeigen, wie sich diese allgemeinen Befunde in der unternehmerischen Realität der Chemieunternehmen widerspiegeln und zwar am Beispiel der leitenden Angestellten im Bereich der FuE sowie der Produktion.

Auf der Ebene der Wissenschaftler und Ingenieure im Bereich FuE der Chemieunternehmen erweitert sich einmal der fachliche Kern der Arbeit. Informationstechnologisches wie molekularbiologisches Wissen gewinnt an Relevanz und ergänzt die hochspezialisierten Detailkenntnisse. Diese Erweiterung ist Vorraussetzung für die individuellen Arbeitsvollzüge, sie ist parallel dazu aber auch grundlegend für die veränderten Kooperationszusammenhänge in den Projektgruppen. Damit ist eine zweite wesentliche Veränderung im Anforderungsprofil bezeichnet, die auf der kooperativ-kommunikativen Ebene zu verorten ist. Wissenschaftlicher Arbeit ist ein kollektiver Charakter inhärent, d.h. Kooperation und Kommunikation in Form vielgestaltiger und kontinuierlicher Kontakte gehört innerhalb der scientific community zum Arbeitshandeln. Entscheidend ist von daher nicht die

\footnotetext{
${ }^{139}$ Baethge, Baethge-Kinsky 1998; Kurz 2002; Schumann 2002; Hollmann et al. 2002.

${ }^{140}$ In diesem Zusammenhang sei auf die Debatte zum Wandel von bürokratisch-hierarchisch strukturierten Organisationen zu lernenden Organisationen hingewiesen. Lernende Organisationen sind durch die Fähigkeit und Bereitschaft gekennzeichnet, bisherige Routinen zu hinterfragen und neue Handlungs- und Entscheidungsmöglichkeiten zu entdecken (stellvertretend für viele: Willke 2001; Lehner 2000).
} 
Tatsache, dass im Rahmen der Projektgruppen ein wesentlich heterogeneres Akteursspektrum miteinander kommunizieren muss. Die kommunikativ-kooperativen Anforderungen an die Beteiligten werden somit ergänzt um die Fähigkeit zur fach- und funktionsübergreifenden Kommunikation mit dem Ziel, die unterschiedlichen Wissensbestände zu verknüpfen. Dabei gilt es, ganz unterschiedliche Geschäftslogiken zu integrieren, differierende Fachsprachen aufeinander abzustimmen und komplexe Sachverhalte für andere Fakultäten verstehbar und anschlussfähig zu machen. Als dritte neue Anforderung wird innerhalb der Projektgruppen die Fähigkeit zur Selbstorganisation und Selbststeuerung wesentliche Arbeitsgrundlage. Nun dominieren in diesem Feld des akademischen Qualifikationen und Expertenwissen von geprägten Typus von Arbeit ohnehin die individualistischen Arbeitsanteile (Selbststeuerung, Selbstregulierung und Selbstintegration). Diese waren aber zumeist in eine sehr streng formalisiert Forschungsorganisation eingebunden. Teilziele und Problemstellungen wurden hierarchisch und funktionsteilig von einigen wenigen Entscheidungsträgern definiert, und so wurde denn auch die industrielle FuE nicht von ungefähr als „Boxenchemie“ abgetan und stand im Bereich der Wissenschaft als Gegenpol des freien, kreativen Arbeitens im akademischen Bereich. ${ }^{141}$ Der Eigenart und Komplexität der Produktentwicklungsprozesse konnte diese Steuerung des Innovationsprozesses in Anbetracht der geschilderten Wissenschaftsentwicklung immer weniger gerecht werden. Zwar ist das Komplexitäts- und Unsicherheitsproblem gerade im Bereich der pharmazeutischen Innovationsprozesse nicht zu lösen. Für die Unternehmen stellt aber die Projektorganisation derzeit die bestmögliche Alternative zur Komplexitätsreduktion dar, können doch durch das diskursive Korrektiv der Projektteams die Erfolgsaussichten einzelner Projekte wesentlich eher projiziert werden. Darüber hinaus werden auf diesem Weg kollektive Lernprozesse möglich, die durch interaktive Rückkopplungsschleifen in Folge veränderter Kommunikations- und Kooperationsleistungen die Effizienz des Innovationsprozesses deutlich erhöhen.

Bei allen neuen Freiheitsgraden darf nicht übersehen werden, dass die deutlich erweiterten Autonomiespielräume auf horizontaler Ebene mit einer vertikalen Entscheidungsstruktur konfrontiert sind. Die Hierarchie bleibt der Widerpart der Teams (Baecker 1999), und dies verschärft die Belastungssituation der Beschäftigten in nicht unerheblichem Maß. Forciert durch die Ausrichtung am Shareholder Value verschärfen die Unternehmen das interne Controlling und etablieren Benchmarkingprozesse, die nicht nur den Vergleich mit externer Konkurrenz anstreben, sondern auch für Projektteams gelten. Um die effizientesten Projekte so früh wie mögliche herauszufiltern, sind die Teams in ein kontinuierliches, kurzgetaktetes Monitoring und Tracking von Zeit, Kosten und Qualitätskriterien eingebunden.

Die skizzierten Veränderungsdynamiken sind indes nicht nur für die Akteure in den zentralen und dezentralen Forschungs- und Entwicklungsfunktionen folgenreich. Wir haben bereits angedeutet, dass neben Akteuren aus dem Marketing und Controlling insbesondere Vertreter der Produktion zunehmend in früheren Projektphasen mit in die Projektteams einbezogen werden. Der Wandel des Innovationsmodells und die mit ihm verbundene Ausrichtung der Unternehmen auf eine zunehmende Prozessorientierung ist somit für die

\footnotetext{
${ }^{141}$ Dies ist insofern eine unzulässige Stilisierung, als etwa innerhalb der Unternehmen zwischen den Arbeitskulturen der Forschungs- und Entwicklungsabteilungen unterschieden werden muss. Gerade die chemische grundlagenorientierte Forschung basiert auf einem ähnlichen Selbstverständnis wie die akademische (Drews 1998).
} 
Produktion ebenfalls folgenreich. Denn parallel zu den heterogeneren und quantitativ deutlich gestiegenen wissenschaftlichen Herausforderungen, die die Informations- und Biotechnologie an die Akteure im Bereich der FuE stellt, gestaltet sich auch das Umfeld der Produktion zunehmend komplexer. So ist dem Trend zur diversifizierten Qualitätsproduktion ein insgesamt höheres Innovationspotential inhärent. Auf der Ebene der Einzelbetriebe sind deutlich mehr Produkte bzw. Produktvarianten somit aber eine insgesamt zunehmende Produktfluktuation zu bewältigen. Dies bedeutet wesentlich mehr Aufwand für Rezepturerstellung und -verwaltung, verkompliziert die betrieblichen Programm- und Belegungspläne und zieht häufiger Umstellungsprozesse der Anlagen nach sich. Außerdem geht die Umstellung auf höherwertigere Produkte nicht selten mit einer Modernisierung der Anlagen oder zumindest einiger Teile einher. Im Rahmen der Herstellung innovativer Produkte sowie der Nutzung neuer Verfahrenstechnik werden die Innovationsanwender in erheblichem Maß zu Ko-Innovatoren, denn sowohl Produkt als auch Prozess stecken nur allzu oft noch in den Kinderschuhen und beinhalten ein erhebliches innovatorisches Potential. Ziel ist es, die qualitativ besten, kostengünstigsten und am sichersten produzierten Endprodukte zu gewährleisten, um so gleichermaßen die erhöhten Qualitätsanforderungen der Kunden zu erfüllen, der Weltmarktkonkurrenz zu parieren und unter den erschwerten Bedingungen ökologischer Restriktionen zu bestehen.

Im Zuge der insgesamt erhöhten Innovationstätigkeit und der Diffusion der Innovationsaufgabe in alle Unternehmensbereiche wandelt sich das Anforderungsspektrum der an diesen Prozessen beteiligten Akteure in ähnlicher Weise wie für die Arbeitskräfte im Bereich der FuE. Dies gilt einmal auf der Einzelbetriebsebene für die aus der chemischen Forschung kommenden Betriebsleiter. Wir haben in Kapitel 2 zeigen können, dass sie traditionell der „,verlängerte Arm“ der Forschung in die Produktion hinein sind und teilweise selbständig kleinere Forschungsprojekte betreiben. Der Anteil an Projektarbeit steigt in diesem Bereich erheblich, und es gelten die auch für die in der zentralen und dezentralen FuE beschäftigten Wissenschaftler benannten Anforderungen.

Das Zentrum von Restrukturierungsmaßnahmen bezogen auf die Produktion in der chemischen Industrie liegt in vielen Unternehmen im Bereich der Ingenieurtechnik, d.h. betroffen sind vor allem Ingenieure. ${ }^{142}$ Ziel ist es, die unterschiedlichen Fakultäten und Ingenieurbereiche Anlagenplanung, Prozessleittechnik (PLT), Zentralwerkstätten und Ingenieurtechnik (IT) effektiver miteinander zu verknüpfen. Bereits die sozio historische Rekonstruktion der Branchenentwicklung hat gezeigt, dass die Arbeit der Ingenieure, die für die technische Umsetzung der Produktinnovationen zuständig sind, einen entscheidenden Anteil am Unternehmenserfolg hat. Die Ingenieure gewährleisten die Sicherheit der Anlagen und sorgen für eine kostengünstige Erstellung wettbewerbsfähiger Neuanlagen. Sie haben einen hohen Einfluss auf die Anlagenverfügbarkeit und die in den Betrieben zu erzielende Produktivität. Dass die Arbeit dieser Experten damit maßgeblich die Wettbewerbsvorteile und Innovationsfähigkeit von Unternehmen bestimmt, steht außer Frage (Kurz, Mickler 2000). Die veränderten Anforderungen und neuen Problemlagen für die Ingenieurtechnik fasst eine renommierte Unternehmensberatung in ihrem Abschlussbericht für ein deutsches Chemieunternehmen folgendermaßen zusammen:

\footnotetext{
${ }^{142}$ Zeitlich liegen konzeptuellen Überlegungen zu diesen Projekten bereits in den 1980er Jahren, ihre Wirkungen entfalten sie jedoch verstärkt in den 1990er Jahren.
} 


\begin{abstract}
„Die Bedeutung der Ingenieurbereiche wird ohne Zweifel zunehmen; ob dem Unternehmen im Wettbewerbsvergleich daraus Vorteile erwachsen, wird in entscheidendem Umfang davon abhängen, inwieweit die Zusammenarbeit zwischen Chemikern und Ingenieuren gefestigt und verbessert wird. Art und Bedeutung der anstehenden Aufgaben verbieten eine Polarisierung. Ein fortgesetztes Herausstellen der Gegensätze - ,Wir Ingenieure - die Chemiker` oder umgekehrt - ist nicht zweckdienlich. Vielmehr sollte die Zusammenarbeit betont und die Grenzen der Ressorts durchlässiger gestaltet werden. [...] Um sich vom ,auftraggebundenen Dienstleister zum kompetenten und leistungsstarken Partner der Chemiker zu entwickeln, müssen die Ingenieurbereiche in Vorleistungen treten; für einen durchschlagenden Erfolg ist zugleich eine Bewußtseinsänderung in den anderen Disziplinen, insbesondere der Produktion erforderlich.“ (unveröffentlichtes Unternehmenspapier 1987)
\end{abstract}

Der Strukturwandel der Ingenieurtechnik basiert ebenso wie für die FuE auf einer neuen, interfakultativen Kooperation, die eine Verstärkung von über das Tagesgeschäft hinausreichenden Beiträgen zur Lösung ressortübergreifender Probleme ermöglichen soll. An die Stelle des Ressortdenkens wird die Prozess- und Projektorientierung gesetzt, ${ }^{143}$ und ökonomische Aspekte spielen in Zeiten des forcierten internen Benchmarking in allen Maßnahmen eine deutlich größere Rolle als zuvor. Außerdem werden zur Verbesserung der Transparenz und zur Sicherung eines einheitlichen Informationsniveaus innerhalb der Teams wie auch projektübergreifend computerbasierte Wissensmanagementsysteme eingesetzt. ${ }^{144}$ Die neue Querfunktionalität sowie die Dezentralisierung von Verantwortlichkeiten bedeutet für das auf technischem Expertenwissen basierende Profil der Ingenieure eine Redefinition zu mehr Kooperation, Integration und Interdisziplinarität (Kurz, Mickler 2000). Damit wird die gerade in den Ingenieurwissenschaften sehr ausgeprägte Spezialisierung, die sich oft in einem gewissen Stolz auf das eigene Fachexpertentum und einer Abschottung gegenüber auch originär ingenieurwissenschaftlichen Disziplinen ausdrückt, auf eine harte Nagelprobe gestellt. Für die Ingenieure sind es einmal die in Anbetracht der rapiden Miniaturisierung immer kürzeren Entwicklungszeiten, die nicht nur eine unbedingte Flexibilität und die Fähigkeit, das eigene Wissen in Frage zu stellen, noch dringlicher als bisher erscheinen lassen. Darüber hinaus verzahnen sich die Technologien und Komponenten in völlig veränderter Art, so dass Mischund Mehrfachqualifikationen die Regelanforderung werden. Die meist enggefassten Bestände an Spezialwissen müssen von den Betroffenen um systemisches und vernetztes Wissen ergänzt werden, um mit dem Innovationsprozess, der sich zunehmend in Grenzbereichen traditioneller Fachrichtungen abspielt, Schritt halten zu können (Kurz 2002; Kalkowski et al. 1995). Und schließlich muss dieses Wissen auch unter diesen veränderten Rahmenbedingungen im Hinblick auf eine adäquate Problemlösung aktiviert, kommuniziert und umgesetzt werden können, weshalb auch für die Ingenieure gilt: Sozial-kommunikative Fähigkeiten werden im Zusammenspiel der Disziplinen und Geschäftskulturen immer dringlicher. Bornierte, auf das eigene Fach gerichtete Lösungen sind in einem solchermaßen definierten Innovationskontext genauso kontraproduktiv wie Verweigerungshaltungen und Abschottungen (Schumann 2003).

\footnotetext{
${ }^{143}$ Interessant ist, dass dieser durchaus anspruchsvolle Ansatz zunächst in der räumlichen Zusammenfassung der Gruppen, die intensiv zusammenarbeiten müssen, realisiert wird- eine gleichermaßen simple, wie äußerst effektive Vorgehensweise.

${ }^{144}$ Die Mitte der 1990er Jahre startende Einführung von SAP als Workflow-Management-System etwa kann aus dieser Perspektive sowohl als informationstechnologische Fundierung wie als „Enabler“ dieser Entwicklung gelesen werden.
} 
Parallel dazu setzt auch auf der Ebene der direkten Produktion ein schwacher Trend zu einer systematischen, d.h. konzeptionell vereinheitlichten Aufwertung von Produktionsarbeit in Form der Einführung von Gruppenarbeit ein: Einer Umfrage des BAVC zufolge praktiziert im Jahr 1995 jede zwölfte Chemiefirma Gruppenarbeit und erprobt neue arbeitsorganisatorische Konzepte. Darüber hinaus plant jedes siebte Unternehmen die Einführung von Gruppenarbeit in naher Zukunft, und sowohl größere Unternehmen ${ }^{145}$ wie etwa BASF, Bayer und Roche als auch klein- und mittelständische Unternehmen schließen interne Betriebsvereinbarungen über Gruppenarbeit ab (BAVC 1996). Der Verbreitungsgrad von Gruppenarbeit in der Chemie Mitte der 1990er Jahre ist damit ähnlich hoch, wie in anderen Industrien, selbst wenn die durch erhebliche Lücken und unterschiedliche Erhebungsmethoden wie Definitionen gekennzeichnete Datenlage einen exakten quantitativen Vergleich nicht zulässt.

Auf der inhaltlichen Ebene zeichnet sich eine relativ einheitliche und in Richtung selbstorganisierter Gruppenarbeit weisende Konzeptualisierung ab. Gesetzt wird in der Chemischen Industrie auf innovative Ansätze, in deren Zentrum Optimierungshandeln als Querschnittsaufgabe, der Abbau von Hierarchieebenen, eine Reorganisation der Instandhaltungs-, Qualitätssicherungs- und Planungsprozesse stehen. Für die Beschäftigten geht es um deutlich größere Handlungs- und Entscheidungsspielräume sowie eine qualifiziertere und interessantere Arbeit. Schließlich verweisen die Konzepte zumeist explizit auf ein Mehr an „Prozessorientierung“, die Produktion soll unter dieser Perspektive „ganzheitlich restrukturiert, mit indirekten Tätigkeiten und die Grenzen zwischen Führung und Ausführung relativiert“ werden (BAVC 1998:11).

Ansatzweise scheint somit die Ende der 1980er Jahre einsetzende Entwicklung zu einer veränderten Nutzung von Arbeitskraft auf dem Shop floor, die sich im flexibelhochintegrierten Organisationstypus manifestiert, in den 1990er Jahren in der Umsetzung arbeitsorganisatorischer Gestaltungsansätze zu münden. Die Bedingungen dafür sind günstig. Erstens hat die Branche bedingt durch exogene Faktoren (v.a. veränderte Behördenauflagen in punkto Sicherheit und Umweltschutz) umfassend auf den Einsatz von Facharbeitern umgestellt. Zugleich besticht die Industrie zweitens durch einen überdurchschnittlich hohen Anteil an Tätigkeitsstrukturen im Bereich der Systemregulierung, die seit Ende der 1980er Jahre in der Prozessindustrie neuen zusätzlichen Einflüssen unterliegt. Folgenreich ist insbesondere die Umstellung auf digitale Informationsübermittlung. Sie erhöht die Regelungsdichte und die nicht-analoge Darstellung der Prozesse fordert veränderte kognitive Fähigkeiten der Beschäftigten. Programmgestützte und gesteuerte Prozesse lassen sich wegen ihrer zwingenden programmtechnischen Fixierung nicht in völlig stabile und störungsfrei laufende Verfahren umsetzen. Die empirische Testphase ist länger als bisher und erfordert eine sukzessive Optimierung, die auf dem Shop floor zu einer Überlappung von Innovations- und Produktionsgeschehen führt. Das Anforderungsniveau der Arbeitsplätze wandelt sich in Richtung Produktionsfacharbeit, die von den Beschäftigten „,in besonderer Weise jene Notwendigkeit aktiver Teilhabe am Produktionsprozeß [fordert], die in der Geschichte der Produktionsarbeit beileibe nicht selbstverständlich ist.“ (Schumann et al. 1994:590). Zwar müssen diese Entwicklungen nicht mit organisatorischen Innovationen auf dem Shop floor pariert werden, betonten schon die

\footnotetext{
${ }^{145}$ Diese Informationen ergeben sich aus eigenen Unternehmensrecherchen und internen Betriebsvereinbarungen der Unternehmen.
} 
AutorInnen des Trendreport. In der Chemischen Industrie werde die Abkehr von traditionellen Mustern der Arbeitskraftnutzung insbesondere dort vollzogen, wo die verschärften Außenanforderungen rein verfahrenstechnisch nicht zu lösen seien (Schumann et al. 1994). Im Gegensatz zu den 1980er Jahren erfährt jedoch drittens die Neubewertung von Arbeitskraft sowohl auf Ebene der betrieblichen Interessenvertretung als auch seitens des Arbeitgeberverbands positive Unterstützung: 1993 legt die zu dem Zeitpunkt noch unter dem Namen IG Chemie Papier Keramik (IG Chemie 1993) arbeitende Gewerkschaft Gestaltungs- und Regelungsvorschläge in Richtung Gruppenarbeit vor. 1996 schließen der Bundesarbeitgeberverband Chemie (BAVC) und die Gewerkschaft eine Vereinbarung zur „Gruppenarbeit in der Chemischen Industrie“ (BAVC 1996). ${ }^{146}$

Welche Ausstrahlungseffekte das spezifische Innovations- und Produktionsmodell der Chemischen Industrie auf die Umsetzung innovativer arbeitspolitischer Konzepte hat und welche Konsequenzen dies für Anforderungsprofil, Tätigkeitsstruktur und Leistungssituation der Beschäftigten hat, bedarf allerdings der empirischen Prüfung. Am Beispiel der Evaluation eines Pilotprojekts zur Einführung „Prozessorientierter Fertigung im Team“ werden wir relevante Aspekte der Umsetzung analysieren und versuchen, eine Gewichtung der je hemmenden und förderlichen Faktoren innovativer Gestaltungsansätze vorzunehmen. Zentral ist dabei die Frage, welche Variablen in welcher Weise die Nutzung der Arbeitskraft der Beschäftigten für den Innovation- und Produktionsprozess bestimmen, und welche Chancen und Risiken die skizzierten Entwicklungen für Beschäftigte in der Chemischen Industrie beinhalten.

\footnotetext{
${ }^{146}$ Dass dieser arbeitspolitische Schwenk maßgeblich von der zu Beginn der 1990er Jahre einsetzenden Lean Debatte inspiriert ist, scheint außer Frage zu stehen. Ob es der Gewerkschaft tatsächlich gelungen ist, einen den chemiespezifischen Anforderungen gerecht werdenden eigenen arbeitspolitischen Ansatz zu formulieren, darf bezweifelt werden. Sowohl der Sprachgebrauch als auch der Abstraktionsgrad der einschlägigen Broschüren stellen eher einen Minimalkonsens dar, mit dem möglichst alle Bereiche der heterogenen industriellen Struktur abzudecken sind. Hier wirkt augenscheinlich eine Interessenvertretungspolitik nach, die noch immer ein Problem mit der „Bodenhaftung“ im Bereich der Arbeitspolitik hat.
} 


\section{Innovative Arbeitsgestaltung in der betrieblichen Praxis}

In der Chemischen Industrie ist, wir haben dies in Kapitel 3 zeigen können, auf unternehmens-, betriebs- und arbeitsorganisatorischer Ebene einiges in Bewegung geraten. Der Wandel des Innovations- und Produktionsmodells hin zu einer stärkeren Prozessorientierung impliziert veränderte Anforderungsprofile zwar nicht der Gesamtheit der Belegschaften, aber doch einer Vielzahl von Beschäftigten, und eine Neubewertung von Arbeitskraft auf allen Ebenen scheint auch in Chemieunternehmen programmatisch: Innovatorisches Handeln der Beschäftigten wird zur neuen Querschnittaufgabe. Wir werden am Beispiel eines Projekts zur betrieblichen Reorganisation zeigen, welche Merkmale eine prozessorientierte Produktion in der Chemischen Industrie haben kann, und wie in diesem Zusammenhang Beschäftigte systematisch zu manifesten Leistungsträgern werden können. Dazu wird zunächst eine Bestimmung der Projektgenese sowie der zentralen Veränderungsimpulse vorgenommen (4.1). In einem zweiten Schritt werden wir die Umsetzungsstrategie des Unternehmens vorstellen (4.2). Daran anschließen wird sich die Frage nach den veränderten Arbeitsbedingungen und Arbeitswahrnehmungen der Beschäftigten im Kontext innovativer Arbeitspolitik. Zu klären ist, inwiefern sich die in Kapitel 2 analysierte Chemiespezifik bei der Umsetzung eines auf selbstorganisierter Gruppenarbeit basierenden Konzepts auswirkt. Im Zentrum steht die bislang empirisch nur unzureichend geklärte Frage, inwiefern ein ausdrücklich an den Schwachstellen des tayloristischen Produktionsregimes ansetzendes Konzept anschlussfähig für eine Umsetzung in der wissensbasierten Chemieproduktion ist (4.3). ${ }^{147}$ Anhand des fallspezifischen Materials werden wir zeigen, dass die Umsetzungsdynamik selbstorganisierter Gruppenarbeit sowohl von der spezifischen Innovationssituation der Einzelbetriebe (4.4) als auch der Neustrukturierung von Hierarchie und Kompetenzen (4.5) beeinflusst wird.

\subsection{Das Projekt „Prozessorientierte Fertigung im Team“: Genese und Zielsetzun- gen}

Die Chem AG gerät wie die gesamte Branche Anfang der 1990er Jahre in eine Krisensituation, die vor allem den Chemie-Sektor betrifft und die trotz anhaltender Erfolge des Sektors Gesundheit zu einem deutlichen Rückgang des Konzerngewinns um 17 Prozent auf 1,5 Milliarden DM führt (Geschäftsbericht 1992). Im Zuge der Strategien zur Bewältigung der Krise setzt das Unternehmen erstens auf eine Restrukturierung des Portfolioss entlang der Kernkompetenzen. Das Unternehmen bleibt nach wie vor ein integriertes chemisch-pharmazeutischer Konzern, fokussiert seine Aktivitäten aber zunehmend auf den Gesundheits-Sektor: Mit 1,4 Milliarden DM gehen über 40 Prozent des gesamten Forschungsbudgets von 3,2 Milliarden DM in dieses Arbeitsgebiet, ${ }^{148}$ und im Geschäftsbericht heißt es, dass der Anteil des Sektors Gesundheit am Konzernumsatz von jetzt 22 Prozent bis zum Jahr 2000 auf 30 Prozent gesteigert werden soll (Geschäftsbericht 1992). Parallel dazu setzt Chem zweitens auf eine starke Zentralforschung, die einmal Auftragsforschung für die Geschäftsbereiche betreibt, aber zum weitaus größeren Teil grundla-

\footnotetext{
${ }^{147}$ Weitere Hinweise liefert die im SOFI entstandene Studie „Konzepte innovativer Arbeitspolitik“ (Kuhlmann et al. 2004).

${ }^{148}$ Im Verglich dazu werden etwa 18 Prozent für den Sektor Landwirtschaft, 15 Prozent für Informationstechnik, 11 Prozent für Industrieprodukte und jeweils 8 Prozent für Polymere und Organica aufgewendet.
} 
genorientiert arbeitet. Dies scheint im Hinblick auf die rasante Wissenschaftsentwicklung die adäquate Strategie für Chem, um interne Wissensbestände und Ressourcen anschlussfähig für Neuentwicklungen zu halten. Während entsprechend der Relevanz von Forschungs- und Entwicklungsaufgaben die Aufwendungen in diesem Bereich steigen.

Obgleich die Konzernleitung im Geschäftsbericht die langfristige Sicherung der Arbeitsplätze, eine intensive Aus- und Weiterbildung aller Beschäftigten sowie die verstärkte internationale Ausrichtung der Personalentwicklung als wesentliche Ziele formuliert, werden drittens bereits 1992 4,8 Prozent an Personal abgebaut, zwischen 1989 und 1999 sind es insgesamt 29 Prozent. ${ }^{149}$ Betroffen sind indes nicht allein Arbeitskräfte in der Produktion: In einer Restrukturierungsmaßnahme werden die sieben Hierarchieebenen auf vier abgebaut, und umfassende Rationalisierungspotentiale in Management und Verwaltung erschlossen.

Im Ergebnis entsteht so ein international ausgerichteter, auf dezentraler Geschäftsverantwortung und internen Marktbezügen basierender Konzern, der in den 1990er Jahren unter das strategische Primat des Shareholder Value gestellt wird (Kädtler 2003; Becker 2001). Mitte der 1990er Jahre wird dies die offizielle Strategie des Unternehmens, die ergänzt wird durch ein „Synergiemanagement“: ${ }^{150}$

Wir wollen die in unserem Unternehmen vorhandenen Synergie-Potentiale stärker nutzen. Das gilt insbesondere für die Synergien zwischen den Unternehmensbereichen sowie für die Aktivitäten in den Märkten und Regionen. Dabei wissen wir, dass (Chem) mehr ist als die Summe der Unternehmensbereiche. (Leitlinien für die strategische Ausrichtung des Konzerns 1996)

Diese abstrakte Bezeichnung korrespondiert auf organisatorischer Ebene mit einer Matrixstruktur, die auf einer hohen interdisziplinären und fach- wie geschäftsbereichsübergreifenden Kommunikations- und Kooperationsdichte basiert (3.3).

Der Umbau kann als Fortsetzung der bereits Mitte der 1980er Jahre durchgeführten Modifikation der Organisationsstrukturen gelesen werden, der ursprünglich eine Aufwertung der Geschäftsbereiche beinhaltet hatte und nun weiter radikalisiert wird. Unter Beibehaltung der divisionalen Struktur wird der Vorstand seiner Ressortverantwortung enthoben und konzentriert seine Aktivitäten auf die strategische Aufstellung des Unternehmens im Markt- und Wettbewerbsumfeld. Die Zuständigkeiten der einzelnen Vorstandsmitglieder beziehen sich entsprechend dieser Struktur auf einzelne Arbeitsgebiete, und damit auf eine Zusammenfassung von Geschäftsbereichen. Hintergrund ist eine über die Bereichsgrenzen hinausreichende Koordination und Kooperation der Geschäftsbereiche, angestrebt wird eine verstärkte Nutzung von Synergieeffekten. Für die einzelnen Geschäftsbereiche hat dies zur Folge, dass sie nun unmittelbar an den Gesamtvorstand berichten und über eine weiter gefasste Selbständigkeit verfügen. Die Geschäftsbereichsleiter werden zur ersten Führungsebene der Generalisten, die ihnen nachgeordneten Ressortleiter bilden die erste Reihe des funktionalen Managements. Die skizzierten Veränderungen stellen die

\footnotetext{
${ }^{149}$ Alles in allem sinkt der Beschäftigtenstand in sechs aufeinanderfolgenden Jahren von 171.000 im Jahr 1990 auf 142.200 im Jahr 1996 (Geschäftsbericht 1996).

${ }^{150}$ Formal übersetzt steht der Begriff Synergie für jene Energie, die für den Zusammenhalt und die gemeinsame Erfüllung von Aufgaben zur Verfügung steht
} 
Weichen für eine innerhalb des Unternehmens wachsende Autonomie der Geschäftsbereiche. Die Geschäftsbereiche können nun beispielsweise frei wählen zwischen internen und externen Lieferanten sowie eigenständig entscheiden, zu welchen Preisen und mit welcher Priorität sie interne Kunden beliefern wollen.

Darüber hinaus wird auf der Ebene der einzelnen Zentralbereiche seit Mitte der 1980er Jahre der Ingenieurbereich reorganisiert, mit dem Ziel, eine engere Anbindung an die Geschäftsbereiche zu sichern. Denn auch in der Chem AG wächst der zu betreuende Anlagenbestand stetig und sind im Rahmen der fortschreitenden Technisierung und Automatisierung immer komplexere ingenieurtechnische Aufgaben auf allen Ebenen zu bewältigen. Und schließlich stellen die in den 1980er Jahren sich verschärfenden gesetzlichen Sicherheits- und Umweltauflagen wie auch die Eigenverpflichtungen des Unternehmens im Rahmen eines „Responsable-Care-Programms“ erhöhte qualitative und quantitative Anforderungen an die Ingenieurbereiche. Eine besondere Aufmerksamkeit erfährt dabei die Instandhaltung, dem neben der FuE kostenintensivsten Bereich der Chemieunternehmen. Im Zuge einer Restrukturierungsmaßnahme werden 1984 die IT - Ressorts in die Geschäftsbereiche integriert „um die Identifikation mit den Zielen und die partnerschaftliche Zusammenarbeit zu fördern“ (internes Unternehmenspapier 1987:20). Insbesondere die Zusammenarbeit zwischen Chemikern und Ingenieuren soll gefestigt und verbessert werden, indem nun die zur Umsetzung von Innovationen und Verfahrensoptimierung relevanten Akteure auch räumlich zusammengefasst werden. Speziell auf der Ebene der Einzelbetriebe ist dies folgenreich, denn die Betriebsleitung wird fortan weitgehend von einem Chemiker und einem Ingenieur besetzt. Zwar bleibt der „Chemiker“-Betriebsleiter letztlich verantwortlich für den Betrieb und der Ingenieur nach wie vor in der Linie IT, dennoch wird so in besonderer Weise die Kooperation und Kommunikation über betriebliche Probleme und deren Lösung gefördert. Aber auch die Kooperation von Ingenieurtechnik (IT) mit der Prozessleittechnik (PLT) wird durch die Zusammenfassung der beiden Fachbereiche zu einer operativen Einheit forciert, die schließlich 1992 in der Zuordnung eines aus IT und PLT zusammengesetzten Ressorts Technik zu jedem Geschäftsbereich münden, und die mit der Integration der PLT - Betriebsbetreuung in die IT - Ressorts verbunden ist. 1994 wird aus den beiden Bereichen offiziell die Zentralkommission Produktion und Technik (bis 1993: ZK Ingenieurtechnik), eine multifunktionale Einheit, die entlang der Anforderungen der einzelnen Geschäftsbereiche personell flexibel und prozessnah aufgebaut ist.

Mit dieser Redefinition des betrieblichen Leistungsprofils und veränderten Mustern der Funktionsdifferenzierung sind wesentliche Merkmale einer Prozessorientierung auf betriebsorganisatorischer Ebene nachzuweisen, und der Abbau von Hierarchieebenen auf Geschäftsbereichs- und Abteilungsebene deutet an, dass auch die Statusorganisation des Unternehmens in Bewegung geraten ist.

Ausstrahlungseffekte auf die arbeitsorganisatorische Ebene der Einzelbetriebe haben diese Restrukturierungen insbesondere durch die engere Zusammenführung der IT und PLT. Zur Keimzelle des Projekts zur Implementation von Teamarbeit in den Betrieben wird eine vom Vorstand 1993 eingesetzte Projektgruppe, die die „Möglichkeiten zur weiteren signifikanten Senkung der Instandhaltungskosten“ auf der Ebene der Produktion entwickeln soll, und zwar jenseits von direktem Personalabbau. Dieses „klassische“ Mittel der Rationalisierung gilt in der Produktion als ausgereizt und verspricht keine relevanten Einsparungen mehr. Im Gegenteil: Im Hinblick auf die potentiellen Optimierungsmöglichkei- 
ten wird eine weitere Personalreduktion als kontraproduktiv angesehen, da in vielen Betrieben die absolute Untergrenze der Personaldecke erreicht ist. Hier kann der Regelbetrieb mit Mühe aufrecht erhalten werden, und für Optimierungshandeln bleibt schlicht keine Zeit. Dies ist aber, wie wir in Kapitel 3 zeigen konnten, gerade in den zu großen Teilen bereits hochtechnisierten Anlagen eine für die Betriebe nicht unproblematische Situation, überlappen sich doch auf dem Shop floor zunehmend Innovations- und Produktionsaufgaben. Immer öfter werden die Beschäftigten als „Experten der Praxis“ in Innovationsprozesse mit einbezogen. Physische und psychische Belastungen als Folge von Zeitdruck und Personalmangel bieten indes keine günstigen Voraussetzungen dafür, die Wissensbestände der Beschäftigte in den Innovationsapparat zu integrieren.

Nach ersten Recherchen kommt die Projektgruppe zu der Einschätzung, dass „Quantensprünge“ ${ }^{151}$ in punkto Kostensenkung im Bereich der Instandhaltung zu erzielen seien und greift in ihren Überlegungen zunächst doch auf eine Personalreduzierung zurück. Gedacht wird an ein zweistufiges Konzept, in dem die Betriebsleitungen eine „Analyse und Bereinigung des Personalüberhangs“ vornehmen und Aufgaben aus der IT und PLT in die Produktionsmannschaften integrieren. Die Arbeitsgruppe sieht darin die Möglichkeit, Hierarchieebenen zu streichen sowie eine engere Zusammenführung von Produktion, IT und PLT bezogen auf die produktionsinternen Arbeitsstrukturen und Ablaufprozesse zu bewirken. Dieses Ziel soll durch die Einführung von Gruppenarbeit erreicht werden. Dieser erste Entwurf konnte sich zwar nicht durchsetzen, doch das Thema der Reorganisation der IH ist mit dem Scheitern des Projekts keineswegs von der Agenda. Bei aller Kritik bleibt die Grundidee bestehen, dass die Kosten der Instandhaltung sinken müssen.

Etwa zur selben Zeit belegen die Ergebnisse einer vom Personalbereich durchgeführten Analyse, dass im Unternehmen die Fluktuation der jungen Chemikanten drastisch zunimmt. Erhoben werden in der Befragung die Verweildauer der Chemikanten im Betrieb sowie die Nutzung des Ausbildungsniveaus der Chemiefacharbeiter in der betrieblichen Praxis. Viele der jungen Facharbeiter äußern sich unzufrieden darüber, dass ihr beachtliches Fachwissen in der Realität nicht abgefragt wird, und fünf Jahre nach Ende der betrieblichen Erstausbildung hat die Hälfte der Chemikanten das Unternehmen verlassen. „Tolle Ausbildung, aber nicht genutzt...“ bringt es der Leiter des Zentralbereichs Personal auf den Punkt (Expertengespräch P/E2). Für die Chem AG als Unternehmen, das als eines der ersten deutschen Chemieunternehmen auf eine umfassende Aus- und Weiterbildungspolitik der Beschäftigten setzte, ein nicht hinzunehmender Zustand.

Aus diesen beiden Projekten wird schließlich in Kooperation von Personalbereich und Konzernplanung das Konzept „Prozessorientierte Fertigung im Team“ erarbeitet, welches sowohl eine stärkere Prozessorientierung als auch Teamarbeit im Visier hat und eine Veränderung der Arbeits- wie auch der Betriebsorganisation vorsieht. Ziel ist es, mit der neuen Organisationsstruktur

- die Produktivität durch Verbesserung der Organisation und der Arbeitsabläufe zu steigern,

- die Zahl von Schnittstellen zu verringern und die Flexibilität durch interdisziplinäre, prozessorientierte Zusammenfassung von Funktionen im Team zu verbessern,

\footnotetext{
${ }^{151}$ Entlehnt ist dieser Begriff dem Business Reengineering, welches auf eine „Verbesserung um Größenordnungen“ abzielt. So geht es „nicht um geringfügige oder inkrementale Leistungsverbesserungen, sondern um Quantensprünge.“ (Hammer, Champy 1994:50)
} 
- Abstimmungs- und Entscheidungsprozesse durch Bündelung von Kompetenzen und Abbau nicht mehr benötigter Hierarchien zu vereinfachen,

- die Unternehmenskultur durch offene Information und Kommunikation und Erhöhung des Spielraums für eigenverantwortliches Handeln und Entscheiden mitarbeiterorientiert zu gestalten,

- Kontinuierliche Verbesserung durch Nutzung und Förderung des kreativen Potentials der Mitarbeiter zu erreichen,

- die Arbeit der Beschäftigten mit Qualitätssicherungs- sowie administrativen und dispositiven Aufgaben anzureichern,

- Spielräume für Selbststeuerung zu erweitern und

- systematische Qualifizierungen für alle durchzuführen.

1995 schließlich liegt eine Pilotbetriebsvereinbarung zur „Einführung der prozessorientierten Fertigung im Team (Gruppenarbeit)“ vor und ein Umsetzungsplan ist festgelegt.

\subsection{Das Konzept in der betrieblichen Praxis}

Nachdem die Phase der Konzeptausarbeitung durch die Arbeitsgruppe abgeschlossen ist, werden zunächst Pilotbetriebe bestimmt. Zwar ist eine konzernweite Umsetzung geplant, zuvor soll jedoch das Konzept auf seine „Praxisverträglichkeit“ geprüft werden. Von daher sind bei der Auswahl der Betriebe nicht nur alle deutschen Werkstandorte repräsentiert, sondern auch alle technischen Verfahren (Konti- und Batchverfahren, Ein - und Vielstoffbetriebe; modernisierte und ältere Produktionsstätten) repräsentiert. Für jeden Betrieb ist als Prozessbegleitung ein Kernteam bestehend aus der Betriebsleitung und je einem Moderator aus dem Bereich der Konzernplanung und der Personalentwicklung zuständig, das die operative Projektarbeit übernimmt. Zur Steuerung und Abstimmung wird es um Vertreter der Geschäftsbereiche, der lokalen Personalabteilung, des örtlichen Betriebsrats sowie dem Projektleiter ergänzt.

Der Projektablauf unterteil sich in drei Phasen. In der Analysephase geht es darum, entsprechend der konzeptionell vorgesehenen Prozessorientierung den „Kernprozess“ zu identifizieren:

„Der Kernprozess des Betriebs ist die Herstellung von Produkten beginnend bei der Produktionsplanung und endend mit der Abgabe des Fertigprodukte. Der Ablauf des Kernprozesses wird durch Hilfsprozesse unterstützt (z.B. Instandhaltung, Analytik, Logistik). Nur der Kernprozess ist wertschöpfend. Je reibungsloser und störungsfreier er abläuft, um so wirtschaftlicher ist er für das Unternehmen.“ (internes Unternehmenspapier 1997)

Daher gilt als Ziel: „Was wirtschaftlich im Betrieb erledigt werden kann, soll dort auch gemacht werden können“ (internes Papier 1997). Um dies zu garantieren, werden einerseits die Betriebsdaten (Lage, Historie, Produkte, Kunden, Kosten- und Organisationsstruktur etc.) sowie die betrieblichen Prozesse im Normal- und Störungsfall erfasst. Bei Workshops, in Einzelgesprächen und Gruppendiskussionen beschreiben die Beschäftigten aller Hierarchieebenen die Prozesse, Schnittstellen, Kommunikation und Verantwortlichkeiten aus ihrer Sicht mit dem Ziel, die Schwachstellen vor allem der Zusammenarbeit zwischen der Tag und Wechselschicht sowie mit den indirekten Bereichen auszuweisen. 
Vor allem allzu umständliche Benachrichtigungsverfahren sollen mit Hilfe dieser Analyse dem Prinzip der „kurzen Wege“ weichen.

In der anschließenden Konzeptionsphase wird das Gestaltungsziel der „Teamorientierung“ mit einbezogen und eine „neue Qualität, miteinander umzugehen“ angestrebt. Dies bezieht sich nicht allein auf die Produktionsmannschaften, denn neben den Schichten als „Prozessteams“ sollen sich Betriebsmeister, Ingenieure und Analytiker in einem Arbeitsgremium, dem „Betriebsmanagementteam“, zusammenfinden. Und auch die für den jeweiligen Betrieb zuständigen indirekten Bereiche werden nun als Teams zusammengefasst. Je nach betrieblicher Spezifität finden sich dementsprechend „Serviceteams“ aus den Bereichen Instandhaltung, Analytik und Logistik. Darüber hinaus werden für die Produktionsmannschaften Qualifikationen und Weiterbildungen geplant sowie Rotationspläne ausgearbeitet, und die Teams wählen ihre Teamsprecher, für die zusätzliche Schulungen angeboten werden.

In der Erprobungsphase sollen sich die Teams „finden“ und das Konzept „mit Leben gefüllt“ werden. Die neuen Informations- und Kommunikationsstrukturen werden erprobt, Qualifikationsmaßnahmen durchgeführt und der „Normallauf“ von Teamarbeit, d.h. insbesondere die neuen Kommunikations- und Kooperationsstrukturen stehen auf dem Prüfstand.

Das vorgelegte Konzept der Projektgruppe ist als Gestaltungsansatz innovativer Arbeitspolitik für Betriebe der Chemischen Industrie zu verstehen: Die Arbeitskräftenutzung zielt nicht nur auf eine stärkere Aufgabenintegration im engen Bereich der Produktionsaufgaben, vielmehr wird sowohl die horizontale als auch die vertikale Arbeitsteilung gelockert. Dies heißt einmal, dass die interne Hierarchie in den Schichtgruppen aufgehoben wird. Außerdem übernehmen die Beschäftigten nun dispositive Tätigkeiten des Schichtmeisters wie auch der Instandhaltung. Dabei bearbeiten die Beschäftigten kleinere Wartungs- und Instandhaltungsaufgaben nicht nur eigenständig, sondern können auch die ihrem Betrieb zugeordneten Schlosser oder PLT-Handwerker direkt beauftragen. Diese unmittelbare Kommunikation soll den komplizierten Dienstweg vom Beschäftigten zum Schichtführer, Betriebsmeister, Werkstattmeister und endlich zum zuständigen Instandhalter ablösen. Abgerundet wird das Konzept schließlich durch ein Mehr an Partizipation der Beschäftigten. Für die Beschäftigten bedeutet dies neben einem höheren Maß an Funktionsintegration vor allem institutionalisierte Möglichkeiten zur Selbstorganisation in Form von Gruppengesprächen und gewählten Gruppensprechern. Mit der Einführung des KVP schließlich gehört die Verfahrensoptimierung ebenfalls zum Anforderungsprofil der Gruppen. Einer schnittstellen-, d.h. fach- und funktionsübergreifenden Zusammenarbeit der Beschäftigten ist so vom Konzept her der Weg bereitet.

Alles in allem weist das Konzept damit eine hohe Übereinstimmung mit dem Konzept selbstorganisierter, funktionsintegrierter Gruppenarbeit auf, wie es aus den Fertigungsindustrien bekannt ist. 


\begin{tabular}{|c|c|c|}
\hline & $\begin{array}{l}\text { Kennzeichen } \\
\text { selbstorganisierter } \\
\text { Gruppenarbeit }\end{array}$ & $\begin{array}{l}\text { Konzeptansatz } \\
\text { Chemische Industrie }\end{array}$ \\
\hline $\begin{array}{l}\text { Unmittelbare } \\
\text { Produktionsaufgaben }\end{array}$ & $\begin{array}{l}\text { Rotation, hohe Einsatzflexibilität } \\
\text { Ziel: erweiterter Gesamtumfang }\end{array}$ & $\begin{array}{l}\text { Rotation, hohe Einsatzflexibilität } \\
\text { Ziel: Aufhebung der Trennung zwischen Messwart } \\
\text { und Anlagenfahrer }\end{array}$ \\
\hline Indirekte Aufgaben & $\begin{array}{l}\text { Erweiterter Umfang, weitgehende } \\
\text { Rücknahme von Spezialisierung }\end{array}$ & $\begin{array}{l}\text { Erweiterter Umfang, Übernahme von Instandhaltung } \\
\text { und Qualitätssicherung (Auflösung von } \\
\text { Funktionsgrenzen) }\end{array}$ \\
\hline Selbstorganisation & $\begin{array}{l}\text { Erweitert: Planung und Kontrolle } \\
\text { als Aufgaben der Gruppe }\end{array}$ & $\begin{array}{l}\text { Erweitert: Übernahme von administrativen und } \\
\text { dispositiven Aufgaben (Urlaubs-, Freischichtplanung; } \\
\text { Arbeitseinsatz; Kontrolle der Qualifizierungsmatrix) }\end{array}$ \\
\hline Gruppensprecher & $\begin{array}{l}\text { Gleichberechtigtes } \\
\text { Gruppenmitglied; gewählter } \\
\text { Koordinator und Ansprechpartner }\end{array}$ & $\begin{array}{l}\text { Ist nicht Vorgesetzter sondern Teil der } \\
\text { Arbeitsgruppe und arbeitet mit; informiert; vertritt } \\
\text { die Gruppenmeinung nach außen, koordiniert die } \\
\text { Zusammenarbeit mit anderen Gruppen; schlichtet } \\
\text { Konflikte; leitet die Gruppengespräche }\end{array}$ \\
\hline Gruppengespräche & $\begin{array}{l}\text { Freie Themenwahl, eigene } \\
\text { Gestaltung }\end{array}$ & Freie Themenwahl, vorgegebener Rahmen \\
\hline (Schicht)Meister & $\begin{array}{l}\text { Unterstützer der Gruppe; } \\
\text { vermittelt zwischen betrieblichen } \\
\text { Zielen und Gruppeninteressen }\end{array}$ & $\begin{array}{l}\text { Führungsaufgaben beinhalten verstärkt die } \\
\text { Betreuung der Gruppen, Vereinbarung von Zielen, } \\
\text { Unterstützung der Zielerreichung, Beratung beim } \\
\text { KVP; Position der Schichtmeister wird gestärkt, da sie } \\
\text { in erhöhtem Umfang in die betrieblichen Informations- } \\
\text { und Entscheidungsprozesse eingebunden werden }\end{array}$ \\
\hline Leistungspolitik & $\begin{array}{l}\text { Leistung durch Eigeninitiative und } \\
\text { Kreativität }\end{array}$ & $\begin{array}{l}\text { Keine leistungspolitischen Aspekte im Konzept bzw. } \\
\text { flankierend vorgesehen }\end{array}$ \\
\hline $\begin{array}{l}\text { Kontinuierlicher } \\
\text { Verbesserungsprozess } \\
\text { (KVP) }\end{array}$ & $\begin{array}{l}\text { Getragen von der gesamten } \\
\text { Gruppe; Ziel: Optimierung des } \\
\text { gesamten sozio-technischen } \\
\text { Systems }\end{array}$ & $\begin{array}{l}\text { Kontinuierliche Verbesserung durch Nutzung und } \\
\text { Förderung des kreativen Potentials der Beschäftigten }\end{array}$ \\
\hline
\end{tabular}

Tabelle 3: Arbeitspolitischer Konzeptansatz des Unternehmens

Flankiert werden diese Shop-Floor-Maßnahmen von veränderten Aufgabenstellungen und Anforderungen an die oberhalb der Schichten agierenden Akteure. Hier weist das Konzept deutliche Unschärfen auf, die betrieblichen Akteure sollen sich nun als „Team“ verstehen. Ein elaboriertes Anforderungsprofil, dass nicht nur oberflächlich auf den höheren Stellenwert sozial-komuikativer Aspekte verweist, ist hingegen nicht ausformuliert. Für den Shop floor sind die vier wesentlichen Gestaltungsziele selbstorganisierter Gruppenarbeit (Gerst 1998) definiert: Erstens ein breiter Aufgaben- und Funktionsumfang, der sowohl direkte produktive wie indirekte fachliche und dispoitive Aufgaben umfasst, zweitens eine gruppengetragene Selbstorganisation, drittens eine Redefinition der Meisterposition (im Konzept sehr unscharf konturiert) sowie viertens eine stärkere Einbindung der Schichtteams in die betriebliche Organisation durch den Aufbau dauerhafter horizontaler wie vertikaler Kooperations- und Kommunikationszusammenhänge sowie durch die Anbindung an betriebliches Innovations- und Optimierungshandeln. Das Unternehmen versucht insgesamt einen sehr weitreichenden Umbau, der insbesondere die betriebliche Sozialstruktur innerhalb der Schichten betrifft und der auch die Führungskräfte mit neuen 
Anforderungen konfrontiert. Die Anforderungsprofile der betrieblichen Akteure werden zum Teil erheblich erweitert, und zusätzliche fachliche wie sozial-kommunikative Kompetenzen sind gefordert. Die nachstehende Übersicht gibt einen Überblick über die vom Unternehmen definierten Bestandteile von Fach-, Sozial- und Methodenkompetenz, die in der neuen betrieblichen Struktur verstärkt gefordert werden.

\begin{tabular}{|c|c|c|}
\hline Fachkompetenz & Sozialkompetenz & Methodenkompetenz \\
\hline $\begin{array}{l}\text { Wissen in neue Zusammenhänge } \\
\text { stellen }\end{array}$ & Individualität zeigen und anerkennen & $\begin{array}{l}\text { Erarbeitung neuer } \\
\text { Arbeitszusammenhänge }\end{array}$ \\
\hline Denkmuster statt Fakten & $\begin{array}{l}\text { Individuelle Fähigkeiten in die Gruppe } \\
\text { einbringen }\end{array}$ & Beschaffung von Informationen \\
\hline $\begin{array}{l}\text { Lernen an komplexen } \\
\text { Problemstellungen }\end{array}$ & Kooperationsfähigkeit & Methoden zur Problemlösung \\
\hline $\begin{array}{l}\text { Artikulation von Lern- und } \\
\text { Problemlöseprozessen }\end{array}$ & Kommunikationsfähigkeit & Präsentation von Arbeitsergebnissen \\
\hline $\begin{array}{l}\text { Systemdenken und } \\
\text { Zusammenhangsverständnis }\end{array}$ & Konfliktfähigkeit & Zeitmanagement \\
\hline $\begin{array}{l}\text { Bearbeitung von nicht unmittelbar } \\
\text { ausbildungsbezogenen Themen }\end{array}$ & $\begin{array}{l}\text { Vertrauen in die Fähigkeiten anderer } \\
\text { haben }\end{array}$ & Planung von Arbeitsschritten \\
\hline \multirow[t]{3}{*}{ Implizites Wissen aktivieren } & Verlässlich sein & \\
\hline & Empathie & \\
\hline & Zuhören können & \\
\hline
\end{tabular}

Tabelle 4: Neue Schwerpunktsetzung des Kompetenzmodells; internes Unternehmenspapier

Etwa ein Jahr nach Beginn der Reorganisationsphase in den Pilotbetrieben wird das SOFI als unabhängiges Institut von Betriebsrat und Konzernleitung beauftragt, das Projekt zu evaluieren. ${ }^{152}$ Für das Unternehmen ist diese Erhebung aus zwei Gründen relevant. Einmal soll eruiert werden, wie es um die Akzeptanz des Konzepts seitens der Beschäftigten bestellt ist. Das Projektmanagement ist davon überzeugt, dass dieses Projekt nicht gegen, sondern nur mit der Unterstützung der Beschäftigten - und des Betriebsrats - konzernweit umzusetzen ist. Außerdem soll die Umsetzungspraxis in den Betrieben bilanziert werden, um einen Einblick in die laufenden Projekte, ihre Problemlagen und spezifischen Umsetzungswege zu bekommen.

\footnotetext{
${ }^{152}$ Die Evaluation umfasste Vor-Ort Recherchen (je Betrieb zwei bis drei Tage), bei denen mit einem Querschnitt der Akteure des Projekts (vom Anlagenfahrer bis zum Betriebsleiter, n=61), Interviews geführt wurden. Darüber hinaus wurden insgesamt 17 Gruppendiskussionen geführt und in schriftlicher Einzelbefragung (n=84) die subjektive Bewertung des Projekts ermittelt. Außerdem fanden Expertengespräche mit dem Projektmanagementteam $(n=10)$ statt.
} 


\subsection{Arbeitsbedingungen und Arbeitswahrnehmung im Kontext innovativer Ar- beitspolitik}

Die prozessorientierte Ausrichtung der Arbeitsorganisation fokussiert auf eine systematischere, schnellere und effektivere Nutzung, Mobilisierung und Integration von Qualifikationen und Kompetenzen. Technische, funktionale und betriebliche Grenzen werden im Rahmen dieser Reorganisation verschoben und Ressourcen und Potentiale der betrieblichen Akteure neu verknüpft und gesteuert. Diese Veränderungen, die u.a. mit der Einführung von Teamarbeit umgesetzt werden sollen, erfolgen in der Chemie in vielerlei Hinsicht unter günstigeren Bedingungen als in der Fertigungsindustrie (vgl. Kap 2 und 3). Die Chemie muss nicht erst tayloristisch zerlegte Einzelarbeit in ganzheitliche Gruppenarbeit reorganisieren, sondern kann an eingespielte Kooperationen und Teamstrukturen sowie bereits eingeführte komplexere Aufgabenzuschnitte anknüpfen. De facto findet sich in der Chemie mitunter mehr Gruppenarbeit im Sinn vom gemeinsamen Arbeiten, gegenseitigem Unterstützen und hohem Aufeinanderangewiesensein im gefügeartig verschränkten Arbeitsprozess, als in vielen Anwendungsfällen der Fertigungsindustrie. Auch der Radius für selbständiges Handeln ist schon heute oft breit gezogen. Dies heißt freilich nicht, dass hier nicht noch Raum wäre für eine offensivere Nutzung der Human Ressources. In punkto Personal- und Arbeitseinsatzplanung sowie der aktiven Einbindung in die betriebliche Problemlösung und Prozessoptimierung bleiben noch breite Spielräume für eine Arbeitsgestaltung, die gleichermaßen die Arbeit produktiver und attraktiver machen könnte.

\subsubsection{Wenig Dynamik auf dem Shop floor}

Trotz dieser durchaus günstigen Ausgangssituation ist die Reaktion in den Untersuchungsbetrieben eher verhalten.

\begin{tabular}{|l|l|l|l|}
\hline \multicolumn{4}{|c|}{$\begin{array}{l}\text { Frage: Sie arbeiten hier in Teamarbeit. } \\
\text { Was sind Ihre Erfahrungen damit? (n=122) }\end{array}$} \\
\hline & verbessert & nicht verändert & verschlechtert \\
\hline $\begin{array}{l}\text { Die Qualifiziertheit der } \\
\text { Arbeit hat sich... }\end{array}$ & 33 & 63 & 4 \\
\hline $\begin{array}{l}\text { Die Selbständigkeit in der } \\
\text { Arbeit hat sich... }\end{array}$ & 32 & 61 & 6 \\
\hline $\begin{array}{l}\text { Der Abwechslungs- } \\
\text { reichtum der Arbeit hat } \\
\text { sich... }\end{array}$ & 30 & 66 & 5 \\
\hline $\begin{array}{l}\text { Meine beruflichen } \\
\text { Perspektiven haben sich... }\end{array}$ & 12 & 79 & 9 \\
\hline
\end{tabular}

Tabelle 5: Bewertung der Arbeitssituation in Teamarbeit

In wichtigen arbeitsinhaltlichen Dimensionen wie der „Qualifiziertheit“, der „Selbständigkeit,“ dem „Abwechslungsreichtum der Arbeit“ und vor allem in der „beruflichen Perspektive“ hat sich jeweils für die Mehrheit durch Teamarbeit keine nennenswerte Verän- 
derung ergeben. Bedingt durch systematisch durchgeführte Qualifikationsmaßnahmen gilt für immerhin 45 Prozent der Befragten die Qualifiziertheit der Arbeit als verbessert, nur noch ein Drittel beantwortet die Frage nach der höheren Selbständigkeit positiv. Bezogen auf das Betriebsklima fallen die Einschätzungen in punkto Zusammenarbeit mit den Vorgesetzten recht eindeutig aus, die Mehrheit sieht keine Veränderungen (70 Prozent). Das Urteil über die schichtinternen Beziehungen ist da differenzierter, je ein Drittel der Beschäftigten sieht diese als verbessert, unverändert bzw. verschlechtert an. Am kritischsten wird schließlich die Entwicklung der Arbeitsbelastungen beurteilt, denn für beinah drei Viertel aller Befragten hat sich die Belastungssituation verschlechtert. Diese Einzelurteile bündeln sich einigermaßen schlüssig im Gesamturteil: Die Arbeit insgesamt hat sich nach Ansicht der großen Mehrheit nicht verändert, und entsprechend der ambivalenten Veränderungsdynamik sind auch nur ein Drittel der Befragten mit dem aktuellen Stand der Umsetzung von Teamarbeit zufrieden, während immerhin 37 Prozent dezidiert ihren Unmut mit dem Stand der Realisierung äußern.

Dieses Stimmungsbild aus den Prozessteams, das auf eine verhaltene Umsetzungsdynamik verweist, erhärtet sich durch die Ergebnisse der Gruppendiskussionen mit den Schichtmannschaften. Zunächst wurde sehr allgemein nach der Haltung der Beschäftigten zum Projekt gefragt. Die Teammitglieder konnten dazu Punkte auf einer Skala abgeben, deren Skalierung von „bin begeistert“ bis „bin dagegen“ reichte. Im Ergebnis bestätigt sich die eher abwartende Haltung dem neuen Konzept gegenüber. Für die Extremeinschätzung „,begeistert“ oder „dagegen“ entscheidet sich kaum jemand, und auch die Positionen „gut“ (12 Prozent) oder „nicht so gut“ (18 Prozent) werden nur von einer Minderheit gewählt. Die eindeutigen Mehrheiten liegen in den Zwischenpositionen bei „gut, aber zu wenig realisiert“ (29 Prozent) und, mit leicht negativer Akzentsetzung, bei „viel versprochen, wenig realisiert“ (37 Prozent). Fasst man hingegen die skeptischen und negativen Bewertungen zusammen, so dominieren diese mit Vorbehalten belasteten Anteile sogar mit knapp sechzig Prozent.

Der Verlauf der Gruppendiskussionen komplettiert dieses Bild. Zwar waren diese im Ergebnis bedingt durch spezifische betriebliche Konstellationen und damit zusammenhängende Arbeitsprobleme durchaus unterschiedlich. Einig sind sich die Gruppen jedoch, wenn es um die Gesamtbewertung des Projekts geht. Die Majorität der Beschäftigten ist insgesamt eher enttäuscht von der Umsetzung eines Konzepts, dass eine selbständigere und abwechslungsreichere Arbeit versprochen hatte, dies in der Realität aber (noch) nicht einzulösen vermochte. Eine Clusterung der Gesprächsäußerungen nach Themen und der Häufigkeit ihrer Nennungen belegt, dass die Arbeitssituation dabei sehr wohl einige relevante Modifikationen erfahren hat: In allen Diskussionen wird betont, dass sich das Informationsniveau deutlich verbessert hat, gefolgt von einem Zuwachs an Eigenverantwortung und Mitsprache und einer verstärkten Einbindung in betriebliche Prozesse. An dritter Stelle werden die kürzeren Wege, d.h. die unkompliziertere und entformalisierte betriebliche Kommunikation positiv hervorgehoben, und auch die Qualifizierungsmaßnahmen gelten als Verbesserung. Diesen Verbesserungen stehen allerdings auf der anderen Seite auch Verschlechterungen gegenüber, die den Skeptizismus und die abwartende Haltung gegenüber dem Projekt durchaus plausibel machen: Zentral in allen Diskussionen sind die erheblichen Arbeitsbelastungen, die mit der Einführung von Teamarbeit in Verbindung gebracht werden, sowie eine mangelnde Anerkennung der eigenen (Mehr-) Arbeit durch die betrieblichen Vorgesetzten. Außerdem registrieren einige Gruppen eine mit dem Aufbau der Prozessteams einhergehende zunehmende Konkurrenz der Schichten untereinan- 
der, die es so vorher nicht gegeben habe. Die betroffenen Teams verweisen an diesem Punkt auf die Zweischneidigkeit der neuen prozessorientierten Strukturen. So sei es zwar von Vorteil, relevante Informationen über Kennzahlen und Ausbeuteergebnisse zu bekommen. Da diese aber für jede Schicht einzeln berechnet, offen am Informationsbrett aushingen, könnte man nun genau nachvollziehen, welches Team wie „erfolgreich“ sei.

In den Gruppendiskussionen als wichtige Veränderungen hervorgehoben

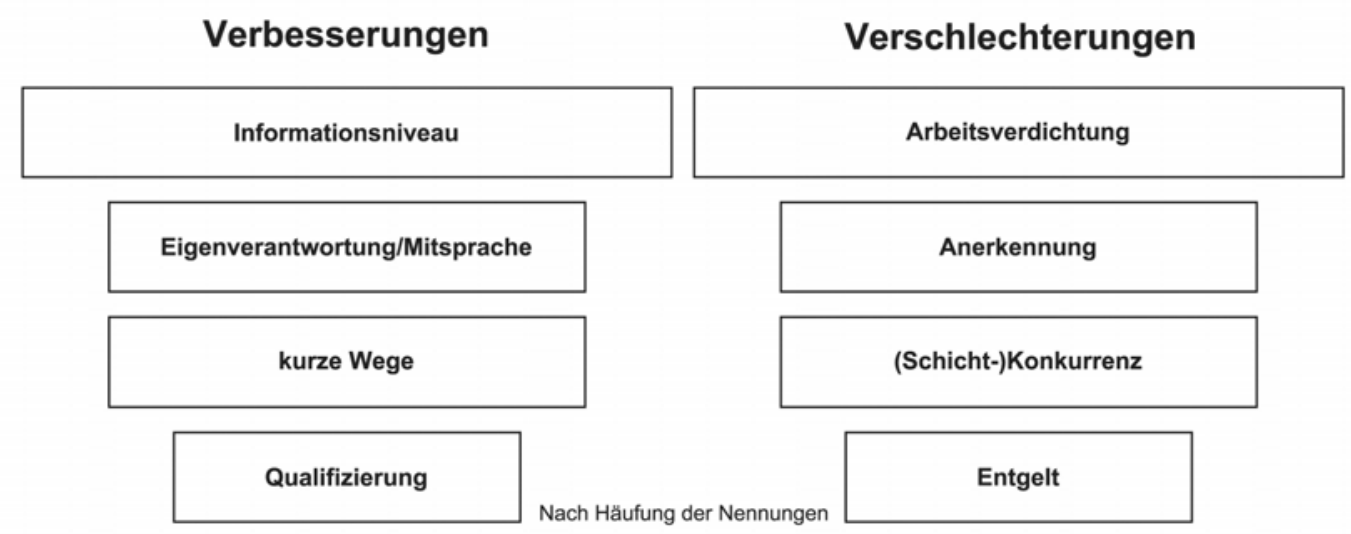

Abbildung 5: Ergebnisse der Gruppendiskussion

Die empirischen Ergebnisse zeigen, dass in den Betrieben durchaus Veränderungsprozesse in Gang gesetzt wurden, diese aber von der Mehrheit der Beschäftigten eher marginal bzw. sogar dezidiert negativ bewertet werden. Die von den Vertretern der Projektgruppe vertretene Maxime der „neuen Zeit der Zusammenarbeit“ scheint damit zumindest auf dem Shop floor kaum Resonanz gefunden zu haben. Dieser Befund ist, betrachtet man Forschungsergebnisse zum Thema Reorganisation, an sich eher überraschungsfrei. Zwar handelt es sich um einen intendierten Wandel, aber „Intendiertheit des Wandels im Fall der Reorganisation heißt, dass er beabsichtigt ist, aber nicht, dass er so wie beabsichtigt realisiert wird“ (Ortmann et al. 1997:333). Das vorgestellte Konzept zur Reorganisation ist eine bewusste, reflexive Re-Strukturation des Handlungsfeldes „Organisation“ durch die beteiligten Akteure. Sie zielt auf eine Modifikation der bestehenden Regeln und Ressourcen und wirkt sich in allen Dimensionen des Sozialen aus, da sie etwa Anforderungsprofile neu bündelt und Kommunikations- und Kooperationsstrukturen verändert. Damit handelt es sich um einen tiefgreifenden Eingriff in etablierte Signifikations-, Legitimations- und Herrschaftsstrukturen (Ortmann et al. 1997). Die interpretative Analyse des in einem spezifischen betrieblichen Kontext vorgenommenen Reorganisationsprozesses muss von daher strukturelle Handlungsbedingungen, ihre Verträglichkeit mit der Innovation und die erwartbaren legitimen Rekurse der Beteiligten und Betroffenen auf diese Strukturbedingungen mit einbeziehen. „Struktur“ wird damit zu einem Schnittpunkt von Gegenwärtigem, hier verstanden als den im Konzept angelegten Veränderungen und Abwesendem, also den tradierten Handlungen der Akteure, deren zugrundeliegende Codes aus „Oberflächenerscheinungen“ abgeleitet werden können (Giddens 1997: 68). Als solche Erscheinungen gelten die in Kapitel 2 vorgestellten Ausprägungen von Kompetenz-, Status- und Kooperationsmodells, in die das Reorganisationsprojekt verändernd eingreift. 
Ihrem Ansatz nach stellt dies für die Akteure einen Eingriff in Routine dar, der darauf zielt, Routine durch Innovation zu ersetzen, um schließlich neue Formen der Routine zu etablieren (Luhmann 2003). Die Mitglieder der Organisation sind nicht passiv und in beschränkter Weise an die Routinen gebunden, sondern bewerten aktiv die ihnen gebotenen Möglichkeiten (Crozier, Friedberg 1977). Dabei legen die Beschäftigten „eine fast instinktive, aber sehr vernünftige Einschätzung der Risiken an den Tag“ (Crozier, Friedberg 1977:242), die im Rahmen des Reorganisationsprojekts zu erwarten ist. Der Prozess der Umsetzung eines in sich in der Regel schlüssigen Konzepts in die organisationale Realität ist somit ergebnisoffen, aber nicht beliebig. Denn: „Sobald die Reformabsicht bekannt wird, wird die Situation unübersichtlich. Es kommt zu Stellungnahmen dafür und dagegen, zu Modifikationen, zu Festlegungen und zu Vorwegnahmen der verschiedensten Art. Es kommt zu Verzögerungen und zu einem Oszillieren zwischen alten und neuen Vorstellungen.“ (Luhmann 2000:333). Bezogen auf unseren Fall bedeutet dies, dass die Belegschaften sehr genau abgleichen, ob der von den Projektplanern behauptete Zusammenhang zwischen Konzeptideen, „Mängeln“ betriebs- und arbeitsorganisatorischer Strukturen und den avisierten Gestaltungszielen mit der Wahrnehmung ihrer Arbeitssituation übereinstimmt.

\subsubsection{Gestaltungsziel Aufgaben- und Funktionsintegration}

Ruft man sich die Strukturen von Chemiearbeit in Erinnerung, steht das Konzept im Hinblick auf die Erweiterung des Umfangs der unmittelbaren Produktionsaufgaben ganz in der Kontinuität der Entwicklung von Chemiearbeit. Ein Charakteristikum von Produktionsarbeit in der Chemischen Industrie, dies haben die vorhergehenden Kapitel gezeigt, ist gerade die team- bzw. technisch-kolonnenartige Kooperation. Arbeitsplatzwechsel und hohe Einsatzflexibilität gehörten hier für einen Großteil der Befragten zumindest in den moderneren Anlagen zum Arbeitsalltag. Bereits Kern, Schumann (1984) konstatierten zwar eine relativ starke interne Arbeitsteilung zwischen Messwarte und Anlagenfahrern, doch werde diese allmählich durch eine „an sich allgemein anerkannte Arbeitseinsatzmaxime horizontaler Flexibilität gelockert, vielleicht aufgegeben“ (Kern, Schumann 1984:263). Und nimmt man die Ergebnisse des Trendreport Rationalisierung, so sind rund achtzig Prozent der untersuchten Betriebe schon deutlich vom traditionellen Organisationsmuster des Produktionszusammenhangs abgerückt und lösen die hierarchische Abstufung unterschiedlicher Regulierungsebenen und -tätigkeiten tendenziell bzw. vollständig auf. ${ }^{153}$ Zwischen Regulierungsarbeiten in der Messwarte und vor Ort wird nur dort noch eine strikte Arbeitsteilung praktiziert, wo es sich in der Frage des Überwachungs- und Interventionshandelns um im Prinzip diskrete Produktionsabschnitte handelt (Schumann et al. 1994:603). Der Anteil an Regulierungstätigkeiten ist dann so gering, dass der Prozessabschnitt entweder gar nicht oder sehr eingeschränkt sowohl von der Messwarte als auch im Feld überwacht und kontrolliert werden kann. Ein spezieller, d.h. räumlich und örtlich gebundener Zugang zum Verfahren wird so notwendig und Spezialisierung gefördert (Schumann et al 1994). Diese Begrenzung der Auflösung arbeitsteiliger Strukturen scheint jedoch eher die Ausnahme, und unsere Befunde belegen: Für die Beschäftigten in den untersuchten Fällen spielen die neuen Möglichkeiten im Bereich der direkten Produktionsaufgaben eine recht geringe Rolle. Das „Neue“ des Neuen hebt sich nicht sonderlich 153 Darunter fallen neben den Betrieben des flexibel-hochintegrierten Organisationstyps auch die flexibel-
teilintegrierten Betriebe (Schumann et al 1994, 603ff.). 
von alten Routinen ab, und in den Interviews wird regelmäßig auf die bestehenden Arbeitsteilungsmuster und Praktiken verwiesen. „Flexibel sind wir hier schon immer“, heißt es oftmals, und die Aufgabenerweiterung wird vor allem als Verregelung bestehender Routinen interpretiert. Diese Umwandlung der informellen Praxis in ein offizielles, verbindliches Element der Arbeitsgestaltung bringt freilich einige Veränderungen mit sich, die für die Beschäftigten wesentlich mehr Aufwand und Regelungsbedarf nach sich ziehen als vorher.

Qualifizierung für einzelne Betriebs- und Anlagenteile erfolgte in den Schichtmannschaften je nach betrieblichen Erfordernissen, Sympathien und/oder persönlichem Engagement. Da das Konzept vorsah, möglichst alle Beschäftigten für alle Anlagenteile zu qualifizieren, wird das für die Chemische Industrie auf dem Shop floor ohnehin charakteristische Anlernen über „Learning by doing“ nun zum zentralen Prinzip erhoben. Die Schichten werden dazu angehalten, Pläne zum Anlernen und Einarbeiten für die gesamte Gruppe zu entwickeln und umzusetzen. Dieses Leitbild der homogenen Gruppe, dass durch selbstorganisiertes Anlernen erreicht werden soll, ist jedoch sehr voraussetzungsreich. Qualifikationsdefizite einzelner Beschäftigter etwa von sogenannten Leistungsgeminderten, älteren Beschäftigten und Beschäftigten ohne Facharbeiterausbildung können auf diesem informellen Weg nicht behoben werden. Die Idee der homogen qualifizierten Gruppe muss als empirisch kaum abbildbarer Idealtypus bezeichnet werden. Zwar treten arbeitsgestalterische Konzepte in der Regel mit diesem Anspruch auf, die Arbeitsrealität zeigte indes, dass Arbeitsteilung und Spezialisierung auch bei selbstorganisierter Gruppenarbeit nicht zu vermeiden sind. So betonte Saurwein (1996), dass eine vollkommene Homogenität nicht nur „nicht erforderlich“ sondern darüber hinaus wegen daraus resultierender Nachteile „nicht sinnvoll“ sei. Während Saurwein vor allem die mit Qualifizierungsmaßnahmen und Höhergruppierungen verbundenen Kosten betont, die durchaus durch eine insgesamt gesteigerte Gesamtleistung der Gruppen aufgefangen werden können, sprechen noch andere Faktoren gegen ein einheitliches Aufgabenprofil. ${ }^{154}$ Einmal sollte auch bei selbstorganisierter Gruppenarbeit ein unterqualifizierter Arbeitseinsatz vermieden werden. Gerade für Arbeitsgruppen in der Chemischen Industrie, in denen es eine starke Hierarchisierung gibt, ist es notwendig, das Know-how etwa von Kolonnenführern durch einen qualifizierten Einsatz zu sichern. Von den Betroffenen wird Gruppenarbeit nicht selten als Statusverlust angesehen, so dass durch den Erhalt der fachlichen Expertise Blockaden der neuen Arbeitspolitik wenn nicht verhindert so zumindest eingeschränkt werden können. Mit der Einführung von Gruppenarbeit werden außerdem Aufgaben aus indirekten Funktionen in die Gruppe integriert, um die Schnittstelle zu den angrenzenden Bereichen aufzuweichen. Ein Teil dieser Aufgaben ist vom Umfang her so gering, dass ein Anlernen oder Qualifizieren aller Beschäftigten kontraproduktiv im Hinblick auf die Qualität der Arbeitsausführung wäre. Denn eine Qualifizierung aller für eine bestimmte Aufgabe ist erst dann sinnvoll, wenn die erworbene Kompetenz regelmäßig abgerufen und eingeübt werden kann. Durch eine Spezialisierung einiger auf diese „Sonderaufgaben“ können die Kompetenzen innerhalb der Gruppe aufgebaut werden, die eine Kooperation mit den vor- und nachgelagerten Bereichen ermöglicht. Und schließlich müssen Tätigkeitsfelder für Beschäftigte mit Einsatzeinschränkungen geschaffen werden. Speziell in diesem Zusammenhang ist das Leitbild des heterogenen Aufgaben- und Funk-

\footnotetext{
${ }^{154}$ Ich folge hier weitgehend der Argumentation von Gerst 1998, der die m.E. wichtigsten Punkte zusammengefasst hat.
} 
tionsprofils wichtig, um zu verhindern, dass innovative Arbeitsformen den „olympiareifen“ Mannschaften vorbehalten bleiben. Aus diesen Gründen folgert Gerst (1998), dass es keine allgemeinen Richtlinien für die Balance zwischen homogenen und heterogenen Anteilen bei Aufgaben- und Funktionsintegration geben kann, sondern fallbezogen am besten in Eigenregie der Gruppe entschieden werden müsse. Dass diese mit der Aufgabenintegration verbundenen Probleme eher eine Randerscheinung denn neuralgischer Punkt bei der Umsetzung sind, liegt an den veränderten Personaleinsatzstrategien des Unternehmens. Die Facharbeiterausbildung ist inzwischen ein Muss für die Beschäftigten und die meisten Un- und Angelernten haben in der Vergangenheit die Zusatzbelastung der Abendschule auf sich genommen und sind auch formell auf Facharbeiterniveau.

Viel stärker wirkt die traditionelle schichtinterne Hierarchisierung im neuen Konzept nach. Wir haben in Kapitel 2 zeigen können, dass diese sich auch über die jeweiligen Arbeitsplätze der Beschäftigten definiert. Die Messwarte und hochtechnisierte Anlagen haben wegen ihres qualifikatorischen Anforderungsprofils einen deutlich höheren Status als die Tätigkeit direkt an den Anlagen. So ist es wenig erstaunlich, dass vor allem die Beschäftigten sich gegen eine umfassende Rotation sperren, die auf diesen Plätzen zu finden sind. Sie haben sich ihren Platz hart erarbeitet und sehen die Rückkehr zu einer Arbeit in der Anlage als gewissen „Abstieg“ an. In einem Fall unseres Samples etwa wird der unterschiedliche technische Modernisierungsgrad der Anlage zum zentralen Knackpunkt für die Bereitschaft zur Rotation. Regelmäßig gibt es Streitereien, weil die Beschäftigten aus der - sauberen, hochmodernen, technisch interessanteren - Anlage nicht in das alte - dreckige, überhitze, stickige - Betriebsteil rotieren wollen. Die Ambivalenz von Formalisierung wird hier deutlich, denn das Arbeitsgestaltungskonzept erlaubt es den Beschäftigten aus dem modernisierten Anlagenteil, ihre durchaus berechtigte Angst vor einem Statusverlust hinter dem neuen Regelwerk zum Arbeitseinsatz zu verbergen. Die Kollegen seien nicht qualifiziert für die Anlage und zum Anlernen habe man aus produktionsbedingten Gründen keine Zeit. So löst ein Teufelskreis von hausgemachten und technischen Lernbarrieren den nächsten ab. ${ }^{155}$ Und auch der umgekehrte Fall ist vorzufinden. Einer Reihe von Arbeitern ist das „Knöpfchen drehen“ in der Messwarte auf Dauer zu langweilig bzw. bedeutet für sie das „ewige Starren auf den Bildschirm“ wesentlich mehr Stress, als in der Anlage zu arbeiten. Sie haben sich in ihrer Arbeit eingerichtet und sehen in der neuen Anforderung des ständigen Lernens und Sich-Qualifizierens eher eine Zumutung denn eine Chance für interessantere Arbeit. Auch in der Chemischen Industrie sind also bei der Umsetzung die individuellen beruflichen Entwicklungen zu berücksichtigen, und Gruppenarbeit bedeutet nicht, dass die Unterschiede bezüglich der Perspektiven und Interessen einzelner Beschäftigter aufgehoben werden.

Ist das Konzept bezogen auf die direkten Produktionsaufgaben eher eine Formalisierung bereits bestehender Praxis, stellt sich die Situation für die indirekten Tätigkeiten deutlich anders dar. Zwar wurde der Instandhaltung bereits seit geraumer Zeit eine hohe Aufmerksamkeit in den anlageintensiven Betrieben des Chemieunternehmens zuteil und suchte das

\footnotetext{
${ }^{155} \mathrm{Zu}$ ähnlichen Ergebnissen kommen auch Faust, Holm: „Gerade die Methode des Anlernens setzt voraus, dass erfahrene Kollegen (...) die Mitarbeiter(Innen) mit den zusätzlich zu übernehmenden Aufgaben vertraut machen (Erklären, Vormachen, Überwachen, Korrigieren). (...) Eine fördernde Haltung durch erfahrene Kollegen ist nicht unbedingt selbstverständlich, drohen doch durch die Förderung (...) die bisherigen Statusunterschiede in der Gruppe nivelliert zu werden, während den erfahrenen Arbeitern aus verschiedenen Gründen der Ausweg in eine neue Distinktion sichernde Arbeitsteilung verbaut sein kann.“(2001:78).
} 
Management hier nach Rationalisierungsmöglichkeiten. Diese blieben jedoch auf die indirekten Bereiche beschränkt und tangierten den Shop floor nicht. Dem Prozessgedanken des Projekts läuft diese Trennung zuwider, und so wurden die Schnittstellen sowohl zwischen Instandhaltung und Produktion als auch innerhalb der Instandhaltung zwischen den einzelnen Gewerken aufgebrochen. Im vorliegenden Fall hat das Unternehmen, wie wir zeigen konnten, mit einer ersten organisatorischen Umgestaltung bereits in den 1980er Jahren begonnen. Das Reorganisationsprojekt bildet in gewisser Weise eine Radikalisierung dieser Überlegungen. Ging es im Kern in der Vergangenheit um die Rationalisierung und Restrukturierung der Prozesse im Bereich der geplanten Instandhaltung (Feststellung eines Schadens, der nicht sofort behoben werden kann oder muss) sowie die Umsetzung von Neuinvestitionen, rücken nun vorbeugende und die ungeplante Instandhaltung in den Mittelpunkt. ${ }^{156}$

Im Bereich der vorbeugende Instandhaltung werden explizit die vorhandenen Potentiale der Beschäftigten genutzt, denn schon die Chemikantenausbildung umfasst Unterrichtseinheiten und Praxisanteile für die Bereiche PLT und Schlosser. (Haftungs-)Rechtliche und berufsgenossenschaftliche Auflagen führten in der Vergangenheit zu einer derart hohen Regelungsdichte, dass das Maß an erlaubten gewerkeübergreifenden Eingriffen der Chemikanten weit hinter ihren fachlichen Qualifikationen zurückblieb. Diesen Qualifikationsüberschuss machen sich die Gestalter des Konzepts zunutze:

„Ich brauche im Grunde genommen nur das Potential der Chemikanten auszunutzen. Aufgrund seiner breiten Ausbildung kann ich ihn betrieblich dazu weiterbilden, dann muss ich dem Mitarbeiter die Kompetenzen dazu geben.“ (Betriebsleiter AI)

Die Beschäftigten bewerten diese erweiterten Möglichkeiten des Arbeitseinsatzes von daher auch durchaus positiv, speziell die Aufwertung ihrer Kompetenzen wird betont. Viele Befragte heben hervor, dass sie nun nicht mehr wegen jedes „Kinkerlitzchens“ die „Spezialisten aus der Werkstatt“ rufen müssen. So hatte es in einem Betrieb beispielsweise lange Wartezeiten wegen mangelnder Verfügbarkeit des PLT-Entstördienstes gegeben. Dieser von den Beschäftigten in der Ist-Analyse beanstandete Punkt ist durch die Qualifizierung der Schichtmannschaften für einfache Schalttätigkeiten behoben.

„'Können' tun wir doch vieles schon lange. Wir haben nur keinen Schein gehabt. Wir haben doch dem Schlosser schon immer geholfen und Sachen vorbereitet, bis der kommt. Aber früher, da durften wir das eben nicht, wenn da was passiert wäre...Heute, mit der Zusatzquali, da muss ich mir dann darüber keine Gedanken mehr machen.“ (Beschäftigter $\mathrm{PH} / 5)$

Dieser facharbeitergerechtere Einsatz schwächt die Milieubedingungen des Angelerntenbetriebs eindeutig ab, und auch Chemiearbeiter ohne einschlägige Facharbeiterausbildung profitieren: Sie bekommen nun die Chance, sich weiter zu qualifizieren. Ein Aspekt, der von manchen Beschäftigten vor allem in der längerfristigen Perspektive relevant wird:

\footnotetext{
${ }^{156}$ Ungeplante Instandhaltung bezeichnet den Prozess vom Bemerken eines Schadens bis zu dessen Beseitigung. Teilprozesse sind Schadensdiagnose, Erteilen des Reparaturauftrags, Koordination von Betriebs- und Reparaturablauf, Einsatzsteuerung (auch von Fremdfirmen) und die Dokumentation der Reparatur.
} 
„Da haben die Leute dann auch was vorzuweisen und müssen nicht so viel Angst haben, wenn mal wieder Arbeitsplätze abgebaut werden.“ (Beschäftigter AI/1)

In unseren Recherchen ist aber auch deutlich geworden, dass diese Befunde in der Regel für die Schlosser- und PLT-Aufgaben gelten. Diese Schnittstelle ist freilich, wir haben dies in Kapitel 2 zeigen können, unter der Hand bereits in vielen Betrieben verflüssigt worden. Ein tatsächlich neues Betätigungsfeld ist aus Sicht der Beschäftigten hingegen die Übernahme einzelner Labortätigkeiten wie etwa Proben zu ziehen und kleinere Analysen zu machen. Hier zeichnet sich für die Chemiekanten ein neues fachliches Betätigungsfeld ab, das die Schnittstelle zum Labor nicht nur durch die eigenständige Ausführung von Laboraufgaben durchlässiger macht, sondern gleichermaßen auf der Ebene der Sozialbeziehungen ein erweitertes Verständnis für betriebliche Abläufe mit sich bringt. Aussagen wie „Jetzt kapier’ ich endlich, wieso die da oben (im Labor) manchmal so lange brauchen mit ihrer Analyse...“ (Beschäftigter HS/4) sind in den Interviews häufig zu hören und bringen das vom Projekt angestrebte erweiterte Verständnis für den prozessualen Charakter der Produktion auf den Punkt.

\subsubsection{Gestaltungsziel Gruppengetragene Selbstorganisation}

Die Arbeitssituation von Chemiearbeitern ist durch eine komplexe Mischung aus hierarchischen und kollegialen Momenten gekennzeichnet, die aus der räumlichen Nähe, gemeinsamem Risiko- und Verantwortungsbewusstsein sowie einer spezifischen betrieblichen Sozialisation, die vor allem durch das Umfeld zwischen großindustriellen und kleinbetrieblichen Strukturen bestimmt ist, resultiert. Dieser team- bzw. gruppenartige Charakter der Produktionsarbeit bildet ein sehr spezifisches soziale Gefüge, in dem sich die Beschäftigten bewegen. Die Schichten sind von daher und wegen ihrer für Gruppenbildungsprozesse optimalen Größe (selten mehr als zwölf Beschäftigte) prädestiniert für selbstorganisierte Gruppenarbeit. Flankiert wird diese Ausgangssituation noch durch einen Arbeitsprozess, der eher gemeinsame Aktivitäten forciert denn Einzelarbeit. Die Messwarte ist nicht nur zentraler Ort der Prozessüberwachung, sondern gleichsam Drehund Angelpunkt sozialer und kommunikativer Aktivitäten. Der für alle Kollegen und Kolleginnen gleiche und die Privatsphäre restringierende Schichtplan sorgt darüber hinaus dafür, dass sich Schichten auch in der Freizeit zu gemeinsamen Aktivitäten treffen, „man kennt sich“ also auf Schicht recht gut. Kommunikation und Kooperation sind auf horizontaler Ebene im Gegensatz zu vielen Bereichen der Fertigungsindustrien ein fester Bestandteil der Arbeitsroutine.

Die Umsetzung des Konzeptbestandteils „gruppengetragene Selbstorganisation“ steht von daher in der Pflicht, wenn schon nicht das originär Neue, so wenigstens den qualitativen Unterschied zur traditionellen Struktur zu belegen. In besonderer Weise gilt dies für die neuen Institutionen der Teamsprecher und Teamgespräche, aber auch für die Regeln der Selbstorganisation, da beides nicht nur an etablierten, sondern dem Konzept sehr ähnlichen informellen Routinen andockt. In unseren Gesprächen ist immer wieder deutlich geworden: Wenig plausibel ist den Schichtmannschaften, was sie mit der neuen Ressource gruppengetragener Selbstorganisation anfangen sollen. Das Neue des Neuen wird vom Gros der Beschäftigten in Abrede gestellt, und vielfach wird es als Zumutung empfunden, dass man nun an Schulungsmaßnahmen „für das Soziale“ teilnehmen soll. Man kennt 
sich, verbringt viel Zeit miteinander, und „miteinander reden, das machen wir doch immer schon, dafür brauchen wir keine Gruppenarbeit“ (Beschäftigter LS/2).

Grundlage der wenig euphorischen Rezeption gruppengetragener Elemente ist aber nicht allein die mangelnde Originalität, sondern begründet sich auch in den spezifischen Konfliktlinien, die aus gruppeninternen Prozessen resultieren. Wo die Schichten bei Urlaubs-, Arbeits-, und Freischichteinteilung in avancierteren Betrieben des flexibelhochintegrierten Organisationstyps ihre eigenen Vorstellungen und Vorschläge äußern konnten, die Entscheidungshoheit aber bei den unmittelbaren Vorgesetzten blieb, muss nun das Team selbst die Entscheidungen treffen. Eine asymmetrische Struktur ist der symmetrischen gewichen. Den Beschäftigten, die zunächst positiv auf diese Verschiebung reagieren, werden die weitreichenden Konsequenzen dieser Entwicklung recht schnell klar:

Mit den AGS und der Urlaubsplanung, das fanden wir schon sehr toll, dass wir das jetzt selber machen können, bis wir dann gemerkt haben, dass man da ganz schön kooperieren muss. Bei uns auf Schicht sind viele verheiratet, da ist es ganz schön schwierig zu bestimmen, wer jetzt darf, wer kann, wer soll. Früher hat das der Betriebsleiter gemacht, da hat niemand gemeckert. Heute gibt es da immer Reibereien. Wir wollen das schon selber machen, wir dachten, dass es gut ist, aber nicht, dass die Umsetzung so schwer ist. (Beschäftigter PH/1)

Wo sich diese Aushandlungen über kooperative Gruppenprozesse einspielen, basieren sie auf eher demokratischen, konsensorientierten Lösungen. Umfassende Regelungen werden festgelegt, die für alle bindend sind. Die vormals oft beklagte Willkür der Vorgesetzen, der sogenannte Nasenfaktor, ist damit ausgeschlossen. Dennoch wird das neue System von manchem Teammitglied als ungleich härter empfunden, da es nun selbstauferlegte und unhintergehbare Regeln und Absprachen gibt. Allemal scheint es leichter, Groll gegen einen schichtexternen Vorgesetzten zu hegen, als sich den in der Gruppe ausgehandelten Gerechtigkeitsvorstellungen zu unterwerfen. Wird gegen erstere auf Schicht noch offen angegangen, sind letztere von allen beschlossen und damit zumindest im Idealfall nur mit überzeugenden Argumenten außer Kraft zu setzen. Inwieweit tatsächlich davon ausgegangen werden kann, dass es sich bei den Aushandlungen in Gruppen um eher diskursive Prozesse handelt (so etwa Kuhlmann, Schumann 2000), oder sich schichtinterne Kräfteverhältnisse reproduzieren, kann an dieser Stelle nicht näher diskutiert werden. Zu bedenken ist, dass Gruppenentscheidungen nicht per se „gerechter“ sind, als die von Vorgesetzten, dass also der Begriff „Gerechtigkeit“ immer nur dem entspricht, was die Gruppe darunter versteht, und nicht einer übergeordneten normativen Maßgabe. Dass es mithin nicht ausreicht, wenn es, wie im zugrunde liegenden Fall, einen Passus im Konzept gibt, der festlegt, dass „Team-Beschlüsse im Einvernehmen (Konsens-Prinzip) getroffen“ werden und „Cliquen-Bildung und Ausgrenzung einzelner unzulässig“ ist, zeigen unsere Ergebnisse: Die recht stabilen sozialen Beziehungen innerhalb der Gruppe werden auf einen harten Prüfstein gestellt, dem sich einige Schichten nicht aussetzen wollen.

„Über so was abstimmen, das geht nicht. Da würde die Gruppe sagen, das muss der Betriebsleiter machen. (Warum?) Das ist ja doch menschlich schon mal so eine Sache. Wir sind ja alles Kollegen hier und das geht ja schon fast ins Private rein. Ich kann ja nicht ein- 
fach über seine Ferien bestimmen, wann er die nehmen darf und wann nicht, das geht doch nicht...“(Beschäftigter PF/5)

Für eine andere Gruppe von Beschäftigten verbirgt sich denn hinter der neuen Aufgabe ein „Preis, den keiner haben will“ (Beschäftigter AI/3), der nun in den Schichten gelandet ist.

„Das ist eine Entlastung für den Schichtmeister, und jetzt klebt es an uns. ,Das schmeiß ich mal in die Gruppe’, das sind dann so die Worte, die vom Schichtmeister kommen.“ (Beschäftigter AI/2)

Die in der wissenschaftlichen Debatte vielfach diagnostizierten Ambivalenzen, die sich für die Beschäftigten im Rahmen „fremdorganisierter Selbstorganisation““157 ergeben, lassen sich auch für die Gruppenarbeit in der Chemischen Industrie feststellen, bleiben aber auf den vergleichsweise kleinen Ausschnitt der beschriebenen Aushandlungsprozesse beschränkt. Die aus den Fertigungsindustrien und dort speziell aus den Bereichen der Montage bekannten Auswirkungen in Form der schärferen gegenseitigen Kontrolle und unsolidarischem Verhalten bilden in unserem Sample eine seltene Ausnahme, und sind, berücksichtigt man die ohnehin eher von solidarischen Momenten geprägte Ausgangssituation von Schichtmannschaften in der Chemischen Industrie, nicht zu diagnostizieren.

\subsubsection{Gestaltungsziel Meister als Unterstützer der Gruppe}

Bereits in Kap. 2 konnten wir zeigen, dass die unterste Führungsposition, die Schichtführer, in der Chemischen Industrie einen speziellen Arbeitskrafttypus verkörpern: Ihre langjährige Betriebszugehörigkeit und das daraus resultierende Wissen um die Prozesse sind wesentlicher Bestandteil ihres Kompetenzprofils und machen sie zum „Führer“ der Schichten im Sinne eines mitarbeitenden Vorarbeiters. In der Regel haben sie die gleichen Qualifikationen wie ihre Kollegen, also keine zusätzliche Meisterausbildung absolviert. Ihr Erfahrungswissen indes prädestiniert sie zur schichtinternen Führungskraft, ihnen wird von daher von der Unternehmensleitung Weisungsbefugnis gegenüber der Schicht erteilt und sie verfügen über einen relativ großen Entscheidungsspielraum (Kern, Schumann 1984). Ihre Anweisungen stehen nicht zur Disposition, sondern müssen ausgeführt werden. Als Schichtführer agieren sie als „bester Mann“ auf Schicht und tragen die volle Verantwortung für den Prozess. ${ }^{158}$ Zusätzlich sind sie als unterste Führungskräfte die Schnittstelle zwischen Tag- und Wechselschicht und nehmen eine wichtige Vermittlungsposition ein. Ihre Bindung an „ihre“ Schichten ist freilich weitaus stärker als ihre Einbindung in das betriebliche Management, und von der Mannschaft wird bei ihnen weniger die Amts- denn die Sachautorität (Sofsky 1989) anerkannt: Die Schichtführer haben das überlegene Fachwissen, das für die Aufrechterhaltung des normalen Arbeitsablaufs unerlässlich ist. Zusätzlich gestärkt wird die Position über eine vom Betriebsmeister ,geliehene Autorität“, und nicht selten sind die Schichtführer verlängerter Arm und Erfüllungsgehilfe des Betriebsmeisters. Insgesamt gilt damit für die Schichtführer ein ähnliches Kompetenzprofil wie für Industriemeister im allgemeinen, ohne dass die Bezeichnung „Meis-

\footnotetext{
${ }^{157}$ Zum Begriff vgl. Pongratz, Voß (1998), für die Diskussion um die widersprüchlichen Wirkungen von Gruppenarbeit vgl. Kapitel 1.

${ }^{158}$ Dieses gilt für allem für die Spät, Nacht und Wochenendschicht, wenn die Tagschicht nicht im Betrieb ist.
} 
ter“ bis Ende der 1980er Jahre üblich wäre. Erst in den 1990er Jahren entstand auch innerhalb der Schichten bezogen auf den qualifikatorischen Hintergrund eine „echte“ Meisterposition, und der Trend zur formalisierten Qualifizierung in Form des IHK-Meisters setzte sich durch. Hintergrund dieser Entwicklung war zunächst ein sehr formaler Aspekt. Die zunehmende Rekrutierung von Chemiefacharbeitern und der beschriebene Wandel der Chemischen Industrie weg von einer Angelerntenbranche änderten zwar wenig am Status der Schichtführer, denn weite Teile der Arbeit blieben geprägt durch ein Erfahrungswissen, welches aus der Präsenz und Erfahrung vor Ort resultiert. In dem Maße, in dem jedoch seit den 1980er Jahren zunehmend Chemiefacharbeiter in den Betrieben die Mehrheit bilden, entsteht auch für die Schichtführer ein Druck zur Aufqualifizierung. Im untersuchten Unternehmen etwa ist inzwischen der mit Abstand größte Teil der Schichtführer IHK-Meister. Das Konzept zur betrieblichen Reorganisation sieht nun eine klare Aufwertung der Schichtführer zu Schichtmeistern vor. Besonders deutlich wird diese Intention am Sprachgebrauch, denn in den Pilotbetrieben wird der Begriff des Schichtführers konsequent durch den des Schichtmeister ersetzt. Die „neuen“ Schichtmeister werden hierarchisch auf eine Stufe mit den Betriebsmeistern gestellt, wenn auch mit „abgeschwächter“ Verantwortung (internes Konzeptpapier). Auch in diesem Punkt scheint das Projekt also eine recht konsequente Fortführung einer ohnehin informell schon bestehenden Praxis darzustellen. Und so ist es wenig überraschend, dass insbesondere die jüngeren Meister mit IHK-Abschluss das Projekt als Chance begreifen, ihre Kompetenzen auch im Bereich schichtübergreifender Belange einzusetzen.

\subsubsection{Gestaltungsziel Einbindung der Beschäftigten in die betriebliche Organisation}

Die veränderte Einbindung der Schichtmannschaften in die betriebliche Organisation basiert auf zwei für die Chemische Industrie wesentlichen Neuerungen, und hier unterscheidet sich das Konzept erheblich von den etablierten Strukturen. Dies betrifft erstens die veränderten bzw. wesentlich vereinfachten Kommunikations- und Kooperationsstrukturen. ${ }^{159}$ Diese zeichnen sich im alten Modell insbesondere durch einen an Hierarchieebenen gebundenen Kommunikationsfluss und davon bestimmte Kooperationszusammenhänge aus. In besonderer Weise betrifft dies die Stör- und Fehlermeldungen, die vom Anlagenfahrer über den Messwart, den Schicht- und Betriebsmeister zum Reparaturmeister des zuständigen Fachgewerks und weiter an die Betriebshandwerker geleitet werden müssen. Die folgende Abbildung zeigt am Beispiel einer Störmeldung, dass der Dienstweg durch eine wesentlich weniger komplizierte Benachrichtigungsform abgelöst wird.

\footnotetext{
${ }^{159}$ An dieser Stelle ist darauf hinzuweisen, dass sich diese „Vereinfachung“ nur darauf bezieht, dass Kommunikations- und Kooperationsstrukturen nun kürzer und direkter sind. Bezogen auf das Projekt insgesamt ist von einem erheblichen Mehr an kommunikativ-kooperativen Bezügen auszugehen, so dass nicht nur die Relevanz von Kommunikation steigt (Minssen 1999), sondern die Strukturen für die beteiligten Akteure auch unübersichtlicher werden. Wir werden später darauf zurückkommen.
} 


\section{„Kurze Wege“"vs. Dienstweg}

Dienstweg „alt“

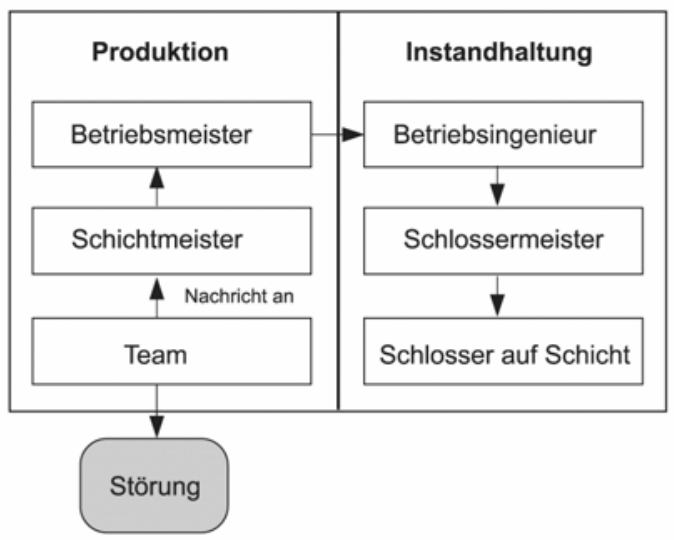

Dienstweg „neu“

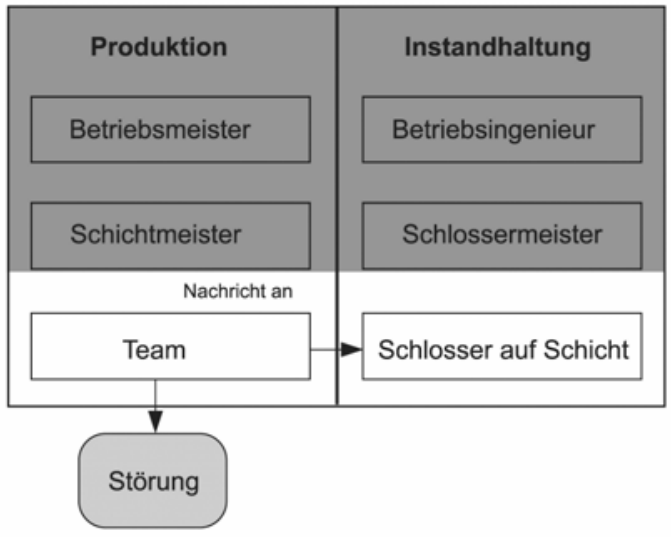

Abbildung 6: Beispiel für neue Kommunikations- und Kooperationsstrukturen

Hier wird in hohem Maß dem Sachverhalt Rechnung getragen, dass in den 1980er Jahren eine stärkere organisatorische Anbindung der Fachgewerke an die Betriebe stattgefunden hat. Viele Beschäftigte aus den Zentralwerkstätten sind in neue, dezentrale Betriebswerkstätten versetzt wurden. Dies führte nicht selten zu der paradoxen Situation, dass auf der horizontalen Ebene die Benachrichtigung der Handwerker durch die Anlagenfahrer innerhalb kurzer Zeit möglich gewesen wäre, da man räumlich eng beieinander arbeitete, zunächst aber die wesentlich weiter entfernten Vorgesetzten eingeschaltet werden mussten. Dass dies im Hinblick auf die chemiespezifisch höheren Risiken auch sinnvoll ist, steht für alle Befragten nicht zur Diskussion. Einhellig sind die Schichten jedoch der Auffassung, dass sie - auch im Eigeninteresse- schon sehr genau wissen, wann ein Prozess bzw. eine Störung kritisch ist, und wann es sich um einen Routinefehler handelt. Informell wird deshalb schon seit längerem der kurze Weg und die direkte Zusammenarbeit praktiziert, allerdings, wir haben dies bereits im Zusammenhang mit der Integration indirekter Zusatzaufgaben in die Schichten betont, macht es für die Beteiligten einen erheblichen Unterschied, nun im legalisierten Bereich der betrieblichen Vorschriften agieren zu können. Schließlich bedeutet dies für sie eine Anerkennung ihres Erfahrungswissens und daraus resultierenden Urteilsvermögens.

Zweitens werden Prozessoptimierung und KVP nun als gruppengetragene Aufgabe definiert. Auf den ersten Blick steht dieser Ansatz in einer recht langen betrieblichen Tradition: Schon 1909 wird im Unternehmen das sogenannte Betriebliche Vorschlagswesen eingeführt, das alle Mitarbeiter des Unternehmens dazu auffordert, Verbesserungen zur Verhütung von Unfällen, dem Betrieb von Geräten, Werkzeugen und Maschinen einzureichen. Dieses gründet jedoch auf einer der Einzelinitiative jedes Beschäftigten und gilt als Teil des betrieblichen Innovationsmanagements (Hauschildt 1999). Seit den 1980er Jahren, wir zeigten dies in Kapitel 3, geraten die Wissensbestände der Chemikanten immer häufiger, wenn auch von Betrieb zu Betrieb durchaus unterschiedlich ausgeprägt, in den Fokus betrieblicher Prozessoptimierungen und werden einzelne Beschäftigte in Innovationsprozesse mit einbezogen. KVP macht nun erstmals Optimierung zur Aufgabe aller 
Beschäftigten. Ein Betriebsleiter fasst treffend zusammen, dass es mit dem neuen Konzept nun darum ginge „systematisch das einzusammeln, was die Leute im Lauf der Jahre angesammelt hätten“ (BL PH/1).

In den Betrieben werden verschiedene Möglichkeiten zur Bearbeitung von KVP-Ideen erprobt, die die Schichtteams in unterschiedlicher Weise mit einbeziehen, und die in der Zielsetzung voneinander abweichen: Im KVP in Projekt- und Fachteams sollen Lösungen für Team-übergreifende Fragestellungen entwickelt werden. KVP in allen Teams im Betrieb richtet sich auf die Verbesserung in kleinen Schritten. KVP in intensiven Workshops unter Beteiligung von Beschäftigten aus verschiedenen Teams schließlich soll „innovatorische Schübe“ durch die Lösung eines komplexen Problems in kurzer Zeit bewirken.

Die mit der Einführung von Gruppenarbeit vom Unternehmen verfolgten Erwartungen, Optimierung „im Kleinen“ mit den Beschäftigten als aktiven Beteiligten zu betreiben, erkennen damit den fachlichen Kern von Chemiearbeit an. Da sich ohnehin viele der Beschäftigten in der Produktion schon allein begründet durch ihr oft langjähriges betriebliches Praxiswissen, das in zunehmendem Maß auf einer soliden Facharbeiter-Ausbildung beruht, als „Experten der Praxis“ verstehen, wird die neue Regelung von ihnen als längst fällige Aufwertung des betrieblichen Status betrachtet.

\begin{tabular}{|l|}
\hline \multicolumn{1}{|c|}{ Zur Prozessoptimierung gehören... } \\
\hline Vorschläge zur Verbesserung der Abläufe \\
\hline Schaffung von Freiräumen im Kernprozess \\
\hline Ermittlung von Tätigkeiten, die in den Kernprozess verlagert werden können \\
\hline Ermittlung von unterstützenden Prozessen, die in den Betrieb verlagert werden können \\
\hline Darstellung der neuen Ablauf- und Aufbauorganisation \\
\hline \multicolumn{1}{|c|}{ Handlungsbedarf festlegen für: } \\
\hline Umsetzen organisatorischer Änderungen \\
\hline Einsetzen von Projektteams zur Lösung besonderer Schwachstellen \\
\hline Fachlichen Qualifizierungsbedarf \\
\hline Einführung des KVP \\
\hline
\end{tabular}

Abbildung 7: Zentrale Merkmale der Prozessoptimierung

Die empirischen Ergebnisse zeigen, dass die Beschäftigten mit überdurchschnittlich viel Engagement bei der Sache sind und Optimierung zur neuen Arbeitsaufgabe wird. Nicht selten wird in den Interviews der „Spaß“ betont, den es mache, die eigenen Erfahrungen und das Wissen um den Arbeitsprozess einzubringen und mit den Kollegen zu diskutieren. Allerdings stehen die chemiespezifischen Strukturen diesen neuen Möglichkeiten nicht selten noch im Weg: Zur Arbeitsroutine der Chemikanten zählt von jeher, dass ihnen enge Restriktionen sowohl bei Ausführung und Dokumentation der Arbeitsschritte als auch im Umgang mit der täglich zu bearbeitenden Datenmenge auferlegt sind. Die Interpretation von Informationen über den Prozessverlauf gilt als Domäne der „Weißkittel“, Meinungen und Erfahrungswerte der Beschäftigten werden nicht oder nur sehr vereinzelt 
zur Kenntnis genommen. So bilanziert denn auch ein Betriebsleiter recht selbstkritisch den Umgang mit den Schichtmannschaften:

„Die Leute müssen unterscheiden lernen, wo sie etwas verändern dürfen und wo nicht. Da sind noch viele Effizienzgewinne zu erwarten, das Potential ist da. (...) Aber man muss bedenken: Die Mitarbeiter sind in diesen Strukturen groß geworden. Man hat ihnen immer enge Grenzen gesetzt, sie sollten immer nur aufschreiben, was verlangt war. Und bloß nicht interpretieren! Das jetzt aus den Leuten rauszubringen, kein Wunder, das ist schwer, vor allen Dingen aus den Älteren.“(BL PH/2)

Im Bereich der Optimierungstätigkeiten wie der Struktur der „kurzen Wege“ zeichnet sich damit im Gegensatz zu den anderen Konzeptbestandteilen eine qualitativ neue Anforderung an das Profil der Chemikanten ab. Zugleich steht damit aber auch in besonderer Weise das traditionelle Kooperations-, Status- und Kompetenzmodell der Betriebe auf dem Prüfstand. Dass zur Unterstützung von Optimierungsaktivitäten der Beschäftigten auch in Chemiebetrieben ein tiefgreifender Wandel des betrieblichen Sozialgefüges notwendig ist, belegen nicht nur unsere Ergebnisse, sondern auch neuere Untersuchungen des SOFI. ${ }^{160}$ Auf die hemmenden und förderlichen Aspekte für diesen Wandel werden wir bei der Darstellung der betrieblichen Konstellationen näher eingehen.

\subsubsection{Projektgestaltung im Urteil der Beschäftigten}

Weisen die Ergebnisse insgesamt auf eine verhaltene Veränderungsdynamik hin, so gilt wie in vielen Projekten der Fertigungsindustrien zur Einführung von Gruppenarbeit (Schumann, Gerst 1997), dass sich die Umsetzung des Konzepts in den einzelnen Pilotbetrieben auf sehr unterschiedlichen Realisierungsniveaus befindet, d.h., dass die Merkmale selbstorganisierter Gruppenarbeit mit ganz unterschiedlicher Reichweite implementiert sind. Differenziert man die Ergebnisse entlang der Umsetzung einzelner Projektbestandteile, zeigen sich durchaus unterschiedliche Entwicklungen, und speziell die Einbindung der Schichtmannschaften in die betriebliche Organisation wird nur in zwei Fällen realisiert. Für ein hohes Realisierungsniveau stehen in unserem Sample die Betriebe PH und AI, in denen nahezu alle Gestaltungsziele zumindest annäherungsweise erreicht werden. Ein Fall unseres Samples (HS) erreicht ein mittleres Realisierungsniveau. In einer ökonomisch zugespitzten Betriebssituation hat die Betriebsleitung eine auf dem Shop floor bereits seit längerem gestartete Integrationspolitik konsequent weitergeführt. In Absprache mit und unterstützt durch die Beschäftigen wurden aber nur einige Elemente des Konzepts umgesetzt. Konkret bedeutet dies, dass sich der Betrieb bei der Arbeitsgestaltung ganz auf die Aufgabenintegration und entsprechende Qualifizierungsmaßnahmen konzentriert hat und die Beschäftigten eng in die Prozessoptimierung eingebunden werden. Da die Schichtgrößen in den letzten Jahren infolge einer erheblichen Personalreduk-

\footnotetext{
${ }^{160}$ In den 1990er Jahren setzte sich unter dem Label Kaizen bzw. KVP in der deutschen Industrie der Trend durch, dass aus Beschäftigten im Rahmen „partnerschaftlicher Rationalisierungsansätze (Tropitsch 1994) „Mitdenker und Mitgestalter“ werden sollen. In der Nomenklatur der sozialwissenschaftlichen Debatte hieß dies, aus Objekten Subjekte der Rationalisierung zu machen. Dass diese „Subjektivierung“ nicht ohne einen weitreichenden Wandel der Arbeits- und Betriebsorganisation auskommen kann, darauf insistiert auch die Managementliteratur. Allerdings zeigt sich in empirischen Studien immer wieder, dass gerade in diesem Punkt die Umsetzung der Gestaltungskonzepte unvollständig bleibt. Vgl. für die Automobilindustrie Kuhlmann (2003), sowie für die Chemische Industrie Kuhlmann et al. (2004) sowie Rönnburg (2003), der sich auf Basis der Erhebungen des SOFI in seiner Diplomarbeit mit dem Thema „KVP in der Chemischen Industrie“ beschäftigt.
} 
tion auf sechs Beschäftigte pro Mannschaft gesunken sind, und die Kommunikations- und Kooperationsstrukturen von allen Beteiligten sowohl als funktions wie hierarchieübergreifend wie auch als direkt und offen beschrieben wurden, wurde die Etablierung von Gruppenstrukturen nicht für notwendig erachtet. Auf niedrigem Realisierungsniveau schließlich sind drei Betriebe einzuordnen, in denen allenfalls geringfügige Modifikationen der Arbeitsorganisation zu beobachten sind. Die Arbeitsstrukturen haben sich kaum verändert und die gruppengetragene Selbstorganisation existiert vor allem als Formalie. Beiden Abstufungen ist jenseits der Differenzen gemeinsam, dass es auf der Ebene der Meister sowie auch des betrieblichen Managements kaum Bewegung gegeben hat. Wir werden später noch auf diesen Punkt zurückkommen.

Differenziert man die Ergebnisse der von uns untersuchten Betriebe nach dem Realisierungsniveau, so sind die voneinander abweichenden Realisierungsniveaus für die Projektbeurteilungen durch die Beschäftigten durchaus relevant. ${ }^{161}$

\footnotetext{
${ }^{161}$ Wir konzentrieren uns im folgenden auf Ergebnisse, bei denen die Unterschiede zwischen den Realisierungsniveaus besonders ausgeprägt sind.
} 


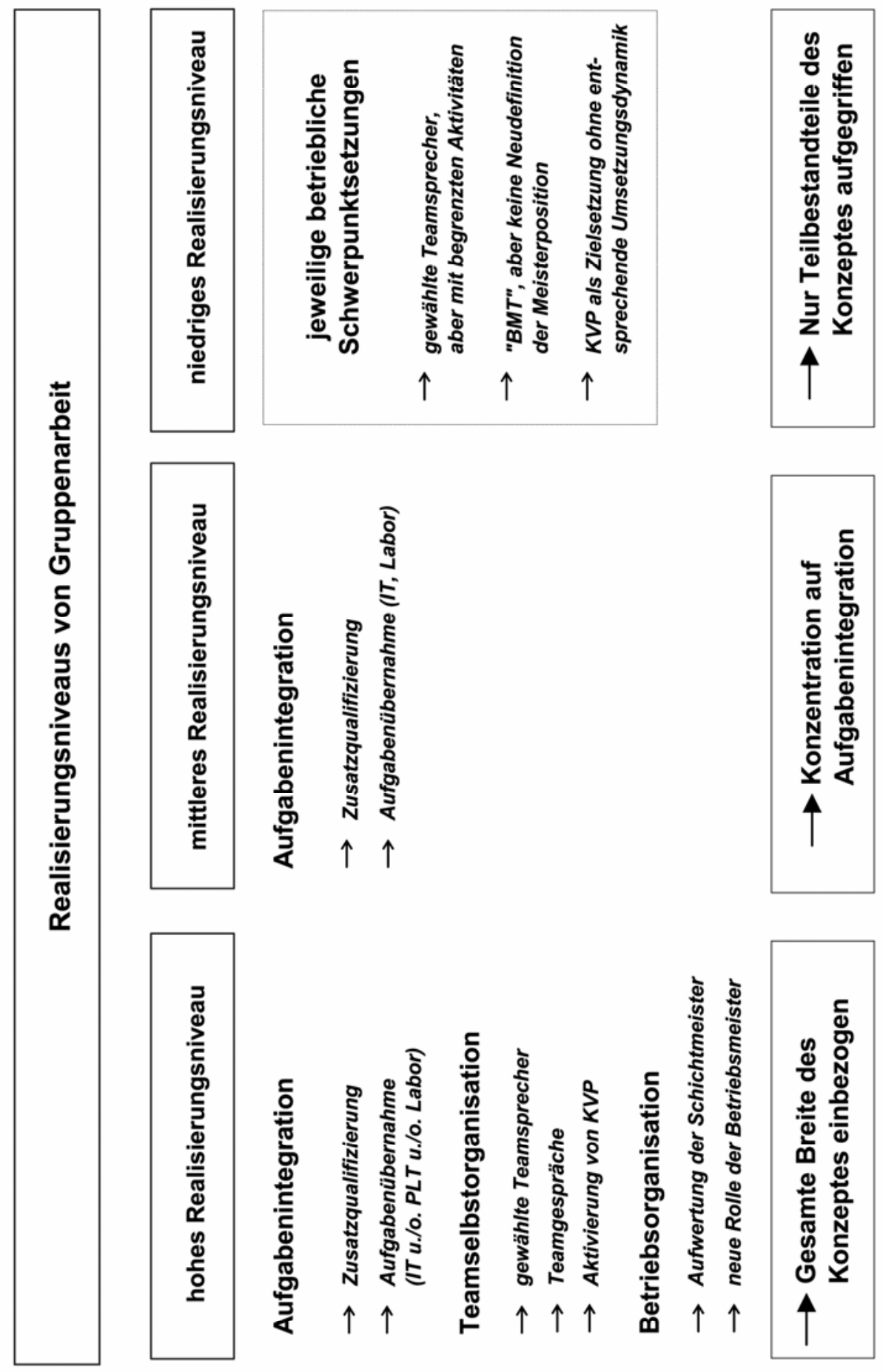

Abbildung 8: Realisierungsniveaus von Gruppenarbeit 
Bei einem hohen Realisierungsniveau werden die beiden wichtigsten Veränderungen, d.h. der Ausbau der Qualifikation (59 \%) und die Erhöhung der Selbständigkeit (48 \%) von den Mannschaften überwiegend positiv aufgegriffen. Das Klima unter den Kollegen gilt der übergroßen Mehrheit als verbessert (72\%). Die Situation auf mittlerem Realisierungsniveau ist geprägt von den Folgen der umfassenden Aufgaben- und Funktionsintegration. Hervorgehoben wird von den Beschäftigten insbesondere die erhöhte „Qualifikation“ (80\%), während die „Selbständigkeit“ unverändert (67\%) gilt. Und auch das „Klima“" erscheint von der Veränderung eher nicht betroffen (70 \% unverändert). Bei niedrigem Realisierungsniveau schließlich kann das Urteil kaum überraschen: „Qualifikation“ (74 \%) und „Selbständigkeit“ (69\%) gelten der Mehrheit als unverändert. Enttäuschte Erwartungen und vergebliche Bemühungen scheinen sich hier indes auf das „Klima zwischen den Kollegen“" auszuwirken und verschlechtern es (57\%) merklich.

\begin{tabular}{|c|c|c|c|c|}
\hline \multicolumn{5}{|c|}{$\begin{array}{l}\text { Frage: Sie arbeiten hier in Teamarbeit. } \\
\text { Was sind Ihre Erfahrungen damit? }(n=122)\end{array}$} \\
\hline & & $\begin{array}{l}\text { hohes } \\
\text { Realisierungsniveau } \\
n=37\end{array}$ & $\begin{array}{l}\text { mittleres } \\
\text { Realisierungsniveau } \\
\mathrm{n}=10\end{array}$ & $\begin{array}{l}\text { niedriges } \\
\text { Realisierungsniveau } \\
\mathrm{n}=37\end{array}$ \\
\hline $\begin{array}{l}\text { Die Qualifiziertheit der } \\
\text { Arbeit hat sich... }\end{array}$ & $\begin{array}{l}\text { verbessert } \\
\text { nicht verändert } \\
\text { verschlechtert }\end{array}$ & $\begin{array}{l}59 \\
41\end{array}$ & $\begin{array}{l}80 \\
20\end{array}$ & $\begin{array}{l}20 \\
74 \\
6\end{array}$ \\
\hline $\begin{array}{l}\text { Die Selbständigkeit in } \\
\text { der Arbeit hat sich... }\end{array}$ & $\begin{array}{l}\text { verbessert } \\
\text { nicht verändert } \\
\text { verschlechtert }\end{array}$ & $\begin{array}{l}48 \\
48 \\
3\end{array}$ & $\begin{array}{l}33 \\
67\end{array}$ & $\begin{array}{l}17 \\
69 \\
14\end{array}$ \\
\hline $\begin{array}{l}\text { Der } \\
\text { Abwechslungsreichtum } \\
\text { der Arbeit hat sich... }\end{array}$ & $\begin{array}{l}\text { verbessert } \\
\text { nicht verändert } \\
\text { verschlechtert }\end{array}$ & $\begin{array}{l}41 \\
57 \\
3\end{array}$ & $\begin{array}{l}90 \\
10\end{array}$ & $\begin{array}{l}19 \\
73 \\
8\end{array}$ \\
\hline $\begin{array}{l}\text { Meine beruflichen } \\
\text { Perspektiven haben } \\
\text { sich... }\end{array}$ & $\begin{array}{l}\text { verbessert } \\
\text { nicht verändert } \\
\text { verschlechtert }\end{array}$ & $\begin{array}{l}22 \\
76 \\
3\end{array}$ & $\begin{array}{l}90 \\
10\end{array}$ & $\begin{array}{l}11 \\
83 \\
6\end{array}$ \\
\hline $\begin{array}{l}\text { Die Belastungssituation } \\
\text { hat sich... }\end{array}$ & $\begin{array}{l}\text { verbessert } \\
\text { nicht verändert } \\
\text { verschlechtert }\end{array}$ & $\begin{array}{l}6 \\
17 \\
78\end{array}$ & $\begin{array}{l}10 \\
90\end{array}$ & $\begin{array}{l}3 \\
32 \\
65\end{array}$ \\
\hline $\begin{array}{l}\text { Das Klima unter den } \\
\text { Kollegen hat sich... }\end{array}$ & $\begin{array}{l}\text { verbessert } \\
\text { nicht verändert } \\
\text { verschlechtert }\end{array}$ & $\begin{array}{l}72 \\
22 \\
6\end{array}$ & $\begin{array}{l}30 \\
70\end{array}$ & $\begin{array}{l}5 \\
38 \\
57\end{array}$ \\
\hline $\begin{array}{l}\text { Die Zusammenarbeit } \\
\text { mit den Vorgesetzten } \\
\text { hat sich... }\end{array}$ & $\begin{array}{l}\text { verbessert } \\
\text { nicht verändert } \\
\text { verschlechtert }\end{array}$ & $\begin{array}{l}23 \\
69 \\
9\end{array}$ & $\begin{array}{l}70 \\
30\end{array}$ & $\begin{array}{l}11 \\
73 \\
16\end{array}$ \\
\hline $\begin{array}{l}\text { Die Arbeit insgesamt } \\
\text { hat sich... }\end{array}$ & $\begin{array}{l}\text { verbessert } \\
\text { nicht verändert } \\
\text { verschlechtert }\end{array}$ & $\begin{array}{l}38 \\
57 \\
7\end{array}$ & $\begin{array}{l}40 \\
50\end{array}$ & $\begin{array}{l}3 \\
78 \\
19\end{array}$ \\
\hline
\end{tabular}

Tabelle 6: Beurteilung der Gruppenarbeit nach Realisierungsniveau

In den Gesamturteilen der Beschäftigten verdichten sich diese Einzeleinschätzungen: Bei hohem Realisierungsniveau gilt zwar die „Gesamtveränderung der Arbeit“ noch als begrenzt (57\% unverändert), aber die Mehrheit äußert doch „Zufriedenheit“ mit der Ent- 
wicklung (58 \%). Bei geringem Realisierungsniveau gilt bald $80 \%$ die Arbeit als „unverändert“ und entsprechend ist eine deutliche Mehrheit explizit „unzufrieden“ mit der Teamarbeit (57\%). Alles in allem bewirkt einzig die auf hohem Realisierungsniveau umgesetzte Reorganisationsmaßnahme für die Beschäftigten auf dem Shop floor einen Neuzuschnitt ihres Anforderungsprofils, der zu einer Aufwertung sowohl im Hinblick auf die Fachlichkeit der Arbeit als auch aufgrund der partiellen Dehierarchisierung ihres betrieblichen Status führt. Das Kompetenzpotential, das sich die Beschäftigten während ihrer Ausbildung sowie in der betrieblichen Praxis angeeignet haben, wird nun in einem umfassenderen Maß durch die konkrete Tätigkeit abgefragt und durch innerbetriebliche Qualifizierungsmaßnahmen weiter ausgebaut. Die Verlagerung planender und steuernder Arbeitsfunktionen in die Schichtmannschaften verändert die interne Handlungssteuerung und verschiebt die Schnittstellen zu betrieblichen Fachbereichen und den unmittelbaren Vorgesetzten. Vor allem letzteres stellt eine erhebliche Veränderung gegenüber dem fachlichen Anforderungsprofil der tradierten Arbeitsorganisation dar. Während vor der betrieblichen Reorganisation schnittstellenübergreifende Zusammenarbeit geduldet aber im strengen Sinn ein Verstoß gegen betriebliche Regeln war, kommt es jetzt zu einem deutlichen qualitativen Bruch, so dass sich die Qualifikationen der Arbeitskräfte und hier vor allem der Chemikanten im Arbeitsprozess durch den Erwerb von Kompetenzen in diesen Bereichen deutlich weiterentwickeln können. Der Wandel des betrieblichen Status der Arbeitskräfte kommt auch in diesen neuen Kommunikations- und Kooperationsbezügen zum Ausdruck.

Als erstes Zwischenergebnis kann festgehalten werden, dass die Beschäftigten das Konzept immer dann kritisch bzw. in der Tendenz negativer bewerten, wenn die Umsetzung der Konzeptbestandteile nur partiell erfolgt. Im Vergleich zur bestehenden arbeitsorganisatorischen Praxis und den damit verbundenen Routinen erwächst aus dem Projekt in diesen Betrieben für die Beschäftigten keine relevante Verbesserung ihrer Arbeitssituation. Es überwiegen im Gegenteil die zusätzlichen Arbeitsbelastungen, die die veränderten betrieblichen Leistungsanforderungen nach sich ziehen, ein Befund, der auch in Gruppenarbeitsuntersuchungen anderer Branchen belegt ist. ${ }^{162}$ Einzig in den Betrieben, die ein hohes Realisierungsniveau erreicht haben, wird seitens der Befragten ein qualitativer Wandel der Arbeitssituation konstatiert. Dabei zeichnen sich die beiden Fälle im Vergleich zu den anderen Betrieben einmal dadurch aus, dass sie zwar nicht alle Gestaltungsziele in Gänze umgesetzt haben, die Gestaltungsbereiche von den betrieblichen Akteuren aber als Einheit verstanden werden, die eng miteinander verschränkt sind. Außerdem wird damit, und dies scheint der qualitative Unterschied zwischen den einzelnen Realisierungsniveaus zu sein, der entscheidend die Beurteilung der Beschäftigten prägt, eine organisatorische Einbindung der Beschäftigten in schichtübergreifende Belange, die in hohem Maß mit Veränderungen betrieblicher Positionen oberhalb der Schichtebene koinzidiert.

Damit stellt sich die Frage, welche Faktoren diese ganzheitliche Umsetzung des innovativen arbeitspolitischen Konzepts im betrieblichen Kontext beeinflussen. Interpretative Ansatzpunkte finden sich in der sozialwissenschaftlichen Literatur, die bereits zu Beginn der 1990er Jahre, als der Reorganisationsboom seinen Höhepunkt erreichte, in empiri-

\footnotetext{
${ }^{162}$ Ausführlich dazu: Gerst et al. 1995; Hollmann, Niemeyer 1998; Minssen 1999; Moldaschl 1994; SenghaasKnobloch et al. 1996.
} 
schen Untersuchungen das Auseinanderfallen von Konzept und Umsetzung belegte. Blockadepolitik von Führungskräften oder betroffenen Beschäftigten sowie die Nichtbeachtung von aus der Reorganisation resultierenden Gewinner/Verlierer - Konstellationen gelten bis heute als schlüssige Erklärungen für dieses Phänomen. ${ }^{163}$ Die Frage nach den Ursachen der Blockadehaltung führt allerdings zu durchaus unterschiedlichen Interpretationen, die über mangelhaftes Projektmanagement, arbeitspolitische Ignoranz des Managements bis hin zu Hinweisen auf strukturell bedingte konkurrierende betriebliche Steuerungsmechanismen und verdeckte Beschäftigteninteressen, die zu Spannungen in der betrieblichen Sozialorganisation führen, reichen (Baethge-Kinsky, Hardwig 2001). Die erstgenannte Interpretation findet sich vor allem in der Management- und Beratungsliteratur und wird als eher technisches Problem formuliert, d.h. die propagierten Managementtools wurden falsch angewendet. Über diese mechanistische Interpretation, die die soziale Komplexität nur unzureichend berücksichtigen, reicht ein Deutungsansatz hinaus, der die Qualität des Reorganisationsprozesses an den Interessen der Akteure misst. Eine Umsetzung der Projektziele ist demzufolge am wahrscheinlichsten, wenn die arbeitspolitische Ausrichtung des Managements möglichst alle divergierenden Interessen miteinander vereinbart. Als wesentliches Erfolgskriterium gilt es, alle Akteursgruppen in die betriebliche Organisation einzubeziehen. So steigen die Möglichkeiten der Beschäftigten, sich aktiv am Prozess zu beteiligen, selbst wenn sie nur mittel- oder langfristig von den Veränderungen betroffen sind, wie etwa die indirekten Bereiche oder Planungsabteilungen (Schumann, Gerst 1998). Eine weitere Ursache der oftmals mangelnden Nachhaltigkeit von Reorganisationsprozessen wird schließlich im latenten Widerspruch zwischen kurzfristig eingeleiteten Rationalisierungsmaßnahmen (Entlassungen, Kurzarbeit, Schließung oder Outsourcing einzelner Betriebsteile) und den nur langfristig zu realisierenden Organisationsentwicklungsprojekten gesehen. Dieser Dissens verunsichere die Betroffenen und forciere eine „Rekonventionalisierung“, d.h. den Rückfall in alte Verhaltensmuster und Rollen (Dörre 1996). Im Anschluss an organisationstheoretische Ansätze ist allen Interpretationen gemeinsam, dass es einen „one best way“ im Sinn einer Rationalität im organisationalen Entscheidungsprozess nicht gibt. ${ }^{164}$ Unterbelichtet bleibt in diesen Ansätzen die Markt-, Produkt- und Innovationssituation der Bereiche, in denen Ansätze innovativer Arbeitsgestaltung umgesetzt werden. Die These, die nun anhand des empirischen Material geprüft werden soll, ist, dass die aus dieser Konstellation resultierende Dynamik, die wir im folgenden als Innovationsaffinität bezeichnen werden, die Projektumsetzung in besonderer Weise zusätzlich positiv wie negativ beeinflussen kann.

\footnotetext{
163 Dörre et al. 1993; Antoni 1994; Schumann et al. 1994; Gerst 1998; Kurz 1999.

164 Organisationstheoretische Ansätze formulierten dies zunächst für Entscheidungsprozesse in Unternehmen (Simon 1957; Simon, March 1958; Hirschman 1974), später wurde dieser Ansatz auch auf die Formulierung von Strategien in Organisationen ausgeweitet ( z.B. Schreyögg 1984).
} 


\subsection{Markt-, Produkt- und Innovationssituation in ihrer Bedeutung für innovative Arbeitsgestaltung}

Vergegenwärtigen wir uns zunächst noch einmal die Entwicklungen auf unternehmensorganisatorischer Ebene, die für die spezifische Markt-, Produkt- und Innovationssituation und die daraus resultierende Innovationsaffinität der Betriebe relevant sind.

Der Wandel von der verbund- zur portfolioorientierten Organisation sowie der Einfluss des Shareholder-Value führt innerhalb der einzelnen Geschäftsbereiche zu einer Konzentration auf profitable, konjunkturrobuste und innovative Geschäftsfelder bzw. Produktionsbetriebe (Dolata 1997). Im Rahmen dieser weitreichenden Reorganisationsprozesse werden bürokratische Strukturen und Hierarchien abgebaut, und auf Geschäftsbereichsebene entstehen multifunktionale Einheiten mit hoher Eigenverantwortung. In der sozialwissenschaftlichen Literatur ist dieser Trend allgemein als Vermarktlichung der innerund zwischenbetrieblichen Beziehungen (Sauer, Döhl 1997) definiert, und zwei unterschiedliche Formen prägen die Diskussion: Einen Pol bildet die Öffnung des Unternehmens oder einzelner Segmente in den Markt, was in seiner extremsten Form die komplette Ausgliederung einzelner Unternehmensteile bedeutet. Die Bildung wirtschaftlich und rechtlich verselbständigter Tochtergesellschaften steht für solche Prozesse. Davon abzugrenzen sind Formen, bei denen die rechtlichen Außengrenzen des Unternehmens nicht angetastet werden. Marktähnliche Steuerungsmechanismen werden innerhalb des Unternehmens aufgebaut und die Unternehmensbereiche als Cost- oder Profit-Center definiert. Profit-Center gelten als „Quasi-Unternehmungen in der Unternehmung“ (Schweitzer 1992, Sp. 2082) und sind objekt- und erlösorientiert. Mit ihren Leistungen müssen sie sich einer internen wie externen Konkurrenz stellen und sind dabei wirtschaftlich (teil)autonom. Die Profit Center-Leiter operieren gewissermaßen wie selbständige Unternehmer und haben dabei weitgehende Entscheidungskompetenz unter der Vorgabe, möglichst hohe Gewinne zu erzielen (Gewinnverantwortung). Zielsetzung des Profit CenterKonzeptes ist die Zuweisung von Ergebnisverantwortung auf dezentrale Geschäftsbereiche. Insgesamt wird so der Unternehmensführung die gewinnorientierte Steuerung der Teilbereichsaktivitäten erheblich erleichtert. Cost-Center hingegen agieren nicht direkt am Markt, sondern sind in interne Kunden-Lieferanten-Beziehungen eingebunden. In ihrem Fall werden ebenfalls Verträge über den Austausch von Leistungen zwischen den Einheiten innerhalb der Wertschöpfungskette geschlossen; dabei wird allerdings kein Marktpreis, sondern Kosten vertraglich fixiert, deren Höhe sich an sogenannten Benchmarks orientieren. Cost-Center haben keine eigene Erfolgs- sondern nur eine eigene Kostenrechnung, und ihre Entscheidungsautonomie ist stärker eingeschränkt als die der Profit-Center (Tullius 1999).

Für das von uns untersuchte Chemieunternehmen nun gilt, dass die Einzelbetriebe in der Regel schon immer als Cost-Center geführt wurden. Der hierarchische Koordinations und Kontrollmodus beinhaltete somit auf Betriebsebene immer auch einen marktlichen Steuerungsaspekt. Für Pilotbetriebe gilt von daher, dass marktbezogene Aspekte eine neue Priorität erhalten, diese aber andere Koordinierungsmechanismen nicht etwa ersetzen, wie es in der Debatte um Vermarktlichung oft - stilisierend - behauptet wird. Erst im Zusammenhang mit dem Portfoliomanagement des Unternehmens kann in etwa beurteilt wer- 
den, wie hart die Marksteuerung durchschlägt. Denn, wir hatten auf diese Spezifität der Chemischen Industrie bereits mehrfach hingewiesen, entscheidend ist in vielen Geschäftsbereichen nicht nur der kurzfristige Return on Investment, sondern vor allem auch die Bedeutung des Einzelbetriebs im Hinblick auf das Gesamtportfolio. Dies kann einerseits heißen, dass etwa im Bereich Pharma Gewinnspannen erst mittel- und langfristig erwartet werden können. Andererseits betrifft dies Betriebe, die zwar im globalen Benchmark schlecht abschneiden, also zu teuer produzieren, die aber als wichtiger interner Lieferant gelten. In dem Fall nimmt das Unternehmen bzw. der Geschäftsbereich die Verluste in Kauf, weil die konstante Qualität sowie die zuverlässige Lieferung für die weiteren Glieder der Wertschöpfungskette prioritär im Verhältnis zum Preis sind. Somit sind die Konstellationen im Unternehmen von Betrieb zu Betrieb äußerst divergent, und Vermarktlichungstendenzen lassen sich für die Produktionsstätten, zumindest für den zugrunde liegenden Fall, recht allgemein fassen als eine teils erhebliche Steigerung des hierarchisch vermittelten Kostendruck seit den 1990er Jahren. Der neue Marktdruck wird insgesamt insbesondere genutzt, um auf allen Akteursebenen eine „Motivation zu eigenverantwortlichem Handeln“ (Arbeitskreis Organisation 1996:628) im Hinblick auf übergeordnete ökonomische Ziele des Unternehmens zu forcieren, wird jedoch nicht als Steuerungsmechanismus dominant. Es gilt vielmehr, auf der gesamtunternehmerischen Ebene die technologische Performanz, die Produktionskosten ebenso wie die Dienstleistungsqualitäten flexibel auszubalancieren.

Parallel dazu wird der Wandel des Innovationsmodells vorangetrieben. Innovation wird zunehmend als Querschnittsaufgabe definiert, und Forschungs-, Entwicklungs-, Instandhaltungs- und Produktionsbereiche kooperieren und kommunizieren in neuartiger Weise miteinander. Zum „zentralen Medium“ dieser Organisation werden projektförmige Arbeitsgruppen, in denen unter Beteiligung aller Akteure aus den betroffenen Teilprozessen Aktivitäten abgestimmt und koordiniert werden. Diese stellen wesentlich kleinere Einheiten als in der traditionellen Funktionalorganisation üblich dar, und machen Beiträge verschiedener Akteure der Prozesskette zur Generierung von Innovationen nutzbar. Auf allen Ebenen werden hierarchie- und fachübergreifende Kooperationsformen verstärkt (Kalkowski, Mickler 2002).

Fasst man diese Entwicklungen zusammen, so sind in den Einzelbetrieben sowohl in wirtschaftlicher, wissenschaftlich-technologischer wie organisatorischer Hinsicht relevante Veränderungen zu bewältigen. Unsere Untersuchungsbefunde belegen, dass sich alle Pilotbetriebe diesen Problemen stellen müssen, diese jedoch von Fall zu Fall eine unterschiedliche Relevanz für die Markt-, Produkt- und Innovationssituation haben. Relevante Dimensionen, um die Innovationsaffinität zu bestimmen, sind

- die betriebsspezifischen Möglichkeiten, Grenzen und Anforderungen für Optimierungshandeln, die insbesondere beeinflusst werden durch den Technisierungsstand des Betriebs, die Zusammensetzung der Produkte, der Reifegrad von Produkt und Technologie, die Marktposition und Spezifika des Betriebs, des Arbeitsgebiets sowie Geschäftsbereichs,

- die betrieblichen Besonderheiten im Hinblick auf die Integration der technischen Serviceeinrichtungen in die Schichtmannschaften,

- die Dichte und Relevanz der Sicherheitsvorschriften,

- die je besonderen Qualifikationsbedingungen,

- $\quad$ und schließlich die Haltung der Betriebsleitung zum Konzept. 
Diese Dimensionen sind entsprechend der äußerst heterogenen Zusammensetzung chemischer Großunternehmen bezüglich der einzelnen Produktionsbetriebe qualitativ unterschiedlich ausgeprägt und folgenreich für die Variationsbreite des Konzepts in der betrieblichen Praxis. Ausgehend von den folgenden Fallbeispielen ${ }^{165}$ können wir zeigen, wie der durch das Projekt intendierte Wandel von der betrieblichen Konstellation beeinflusst ist und damit in bestimmte Verläufe und Trajektorien gezwungen wird. Mittels der oben genannten Dimensionen kann eine „betriebliche Pfadabhängigkeit“ nachgezeichnet werden, die sich aus Notwendigkeiten und „small events“ kombiniert (Ortmann et al. 1997:335). Anhand unserer Befunde lassen sich zwei spezifische Konstellationen unterscheiden.

\subsubsection{Neue Formen der Arbeitsorganisation bei gering ausgeprägter Innovationsaffinität}

Für die betrieblichen Konstellationen PF und KS wurde selbstorganisierte Gruppenarbeit auf niedrigem Niveau umgesetzt. Das betriebliche Sozialgefüge entspricht nach wie vor weitgehend den traditionellen Strukturen. Untersuchen wir zunächst, welche Faktoren für diese Entwicklung maßgeblich sind.

Der Fall PF gehört zum Arbeitsgebiet Landwirtschaft. Es ist durch eine recht stabile Entwicklung sowohl im Bezug auf die Umsätze ,die Betriebsergebnisse sowie eine hohe FuE-Quote gekennzeichnet. Die Fortschritte im Bereich der grünen Biotechnologie haben hier, ähnlich wie im Arbeitsgebiet Gesundheit, eine Dynamisierung im Bereich FuE mit sich gebracht. Während die Forschungsaktivitäten lange Zeit hoch konzentriert in Deutschland stattfanden, werden seit Beginn der 1990er Jahre Forschungszentren in den USA und Japan aufgebaut, um eine direktere Anbindung an die regionalen Pflanzenschutzmärkte zu gewährleisten. Der Betrieb PF ist von diesen Entwicklungen weitgehend ausgenommen, denn keines der Produkte basiert auf neuen biotechnologischen Verfahren oder Erkenntnissen, im Gegenteil: Die 14 unterschiedlichen Insektizide, Fungizide sowie Zwischenprodukte zu deren Produktion, die in diskontinuierlicher Chargenproduktion hergestellt werden, sind durchweg am Markt etablierte, technologisch ausgereifte Produkte. Der Betrieb steht mit dieser Produktpalette im Geschäftsbereich Pflanzenschutz im Untersuchungszeitraum wirtschaftlich einigermaßen gut da, allerdings läuft der Patentschutz für eine Reihe von Produkten demnächst aus, und die Produkte müssen sich im internationalen Wettbewerb behaupten. Gerade im Bereich der Pflanzenschutzproduktion bedeutet dies, das zu dem international niedrigeren Lohnniveau im Ausland meist eine deutlich geringere Kostenbelastung durch Umwelt-Auflagen hinzukommt. Die ausländische Konkurrenz kann von daher wesentlich kostengünstiger anbieten. Eine Kompensation dieser Differenz ist in der Regel nur durch eine qualitativ hochwertigere Fertigung zu erreichen. Für konventionelle Pflanzenschutzmittel spielt jedoch eine qualitative Marktführerschaft nicht die Rolle, wie beispielsweise in dem stark an Qualitätsstandards ge-

\footnotetext{
${ }^{165}$ Wir werden insgesamt nur die vier der sechs Betriebe analysieren, die im Hinblick auf ihre Konstellationen als verallgemeinerbar für die Umsetzung des Projekts in Chemiebetrieben gelten können. Im Fall HS konnten wir aufgrund einer angespannten ökonomischen Situation nur sehr eingeschränkt recherchieren. Hinter dem Fall LS verbirgt sich insofern eine Sondersituation, als es sich um drei Betriebe handelt, die eng miteinander zusammenarbeiten müssen, und die nun durch das Projekt prozessorientierter gestaltet werden sollen. Im wesentlichen fokussierte sich das Projektmanagement auf die Reorganisation des Instandhaltungsbetriebs, dessen Zuständigkeiten seitens der einzelnen Gewerke für die beiden Produktionsbetriebe neu bestimmt werden sollten. In Anbetracht dieser deutlich anderen Ausgangssituation werden wir diesen Fall nicht näher analysieren.
} 
bundenen Bereich der Pharmazeutika. In der Konsequenz bedeutet dies, dass ein Teil der Produktion eingestellt und in absehbarer Zeit (ca. zwei Jahre) die Produktpalette umgestellt sein wird. Erste Vorbereitungen für diese Umstellung sind bereits in Gang: Ein Anlagenteil wird mit einer neuen PLT-Anlage ausgestattet, eine Abfüllanlage erneuert, das Tanklager saniert sowie Umverrohrungen für den Produktanlauf begonnen.

Für den Betrieb ist damit in Bezug auf das Innovations- und Optimierungshandeln der Schichtmannschaften eine komplizierte Gemengelage entstanden: Die alten Anlagen bieten kaum noch Raum für Optimierungen, und selbst wenn es seitens der Beschäftigten Verbesserungsvorschläge im Rahmen von KVP gibt, werden diese zumeist nicht mehr bearbeitet. Die Gründe der Betriebsleitung scheinen relativ offensichtlich: Wozu soll noch optimiert werden, wenn der Output als optimal gilt, die Produktqualität keine Wettbewerbsvorteile erbringt und der Betrieb ohnehin in naher Zukunft auf andere Produkte umstellen wird?

Die Installation der neuen PLT hingegen bleibt das Geschäft einzelner externer Spezialisten (Mitarbeiter der Montage- und Softwarefirmen) sowie der internen PLT-Abteilungen und der Betriebsingenieure. Ansprechpartner aus der Produktion sind insbesondere die Betriebsmeister sowie die Betriebshandwerker. Die Beschäftigten werden nicht systematisch in die kooperativ-kommunikativen Zusammenhänge dieser Spezialisten mit einbezogen. Ihnen bleibt nur die Möglichkeit, Vorschläge in einem „Optimierungsordner“ zu dokumentieren, wenn die Anlage bereits läuft. Aus Perspektive der Betriebsleitung hat dies nicht allein mit einer Nicht-Anerkennung der Kompetenzen der Beschäftigten zu tun. So stellt zwar der extrem niedrigen Facharbeiteranteil von nur 29 Prozent eine Schwachstelle dar, problematischer ist indes die dünne Personaldecke, die kurzfristig zu einer deutlichen Aufgabenerweiterung (aber auch Arbeitsverdichtung) für die Beschäftigten beigetragen hat. Bedingt durch den massiven Personalabbau der vergangenen Jahre wurde bereits ein erklecklicher Anteil von IH-Aufgaben in die Schichten integriert, die Einsatzflexibilität erhöht und eine Rotation eingeführt. So sind denn auch weniger die aktuellen Qualifikationsniveaus der Beschäftigten, sondern die Anforderungen, die sich aus der Umstellung auf die neue PLT ergeben, ein Problem. Alle Beschäftigten müssten an zusätzlichen Qualifizierungen, Aus- und Weiterbildungen teilnehmen. Da die Auslastung der Schichten inzwischen aber bei 100 Prozent liegt, bleibt für diese dringend notwendigen Weiterbildungsaktivitäten keine Zeit.

Der Betrieb KS hingegen wurde erst 1993 aus dem Geschäftsbereich Anorganische Chemikalien herausgenommen und dem Bereich Kunststoffe zugeordnet. Bis zum Zeitpunkt der Untersuchung erfolgt die Anbindung an den neuen Geschäftsbereich ausschließlich über die PLT-Abteilung, ansonsten führt der Betrieb nach Meinung des betrieblichen Managements eine Art „Inseldasein“. Das Verfahren zur Herstellung eines Zwischenprodukts erfolgt vollkontinuierlich, ist komplex und sicherheitstechnisch anspruchsvoll. Die Produktqualität ist in der Regel unproblematisch. Der Betrieb KS hat recht bewegte Zeiten hinter sich: Im Zuge der Straffung der Unternehmensaktivitäten und damit verbundenen Redefinition von Kernkompetenzen und Produktportfolio stand Anfang der 1990er der Betrieb KS als potentieller Kandidat für eine Schließung auf dem Plan, denn der konzerninterne Konkurrent in den Niederlanden produzierte deutlich günstiger. 
Einer Kostensenkung durch Personalreduktion und Ausbeuteverbesserung standen betriebsspezifische Probleme gegenüber. Da der Betrieb bereits seit 30 Jahren produziert, gilt sowohl das Verfahren wie das Produkt im Bezug auf relevante Optimierungspotentiale ausgereizt. Darüber hinaus kann auch kein Personal abgebaut werden, wie dies in anderen Betrieben durchaus üblich ist. KS ist ein Störfallbetrieb, und wegen des hohen Risikopotentials sind aufwändige Dokumentationsverpflichtungen und detaillierte Sicherheitsvorschriften im Arbeitsprozess zu beachten.

Dies hat erhebliche Konsequenzen für die Arbeitssituation. Erstens muss der Anteil der Facharbeiter rechtlich bedingt bei nahezu 100 Prozent liegen. Zweitens muss aus genehmigungsrechtlichen Gründen im Betrieb mehr Personal vorgehalten werden, als für die Überwachung der ruhig laufenden Anlage notwendig ist. Drittens ist die Rotation der Beschäftigten über die Arbeitsplätze seit jeher eingeführt und alle Arbeitskräfte müssen auf allen Plätzen eingesetzt werden können. Der Wechsel wird über Monate im voraus festgelegt. Viertens schließlich sind die Anlagen seitens der PLT so ausgelegt, dass ein individueller Fahrstil kaum möglich und sicherheitsrechtlich nicht erwünscht ist.

Seitens des Betriebs machte man sich diese Besonderheiten in produktiver Weise für die Rationalisierung zunutze. Da die Mannschaften hoch qualifiziert, flexibel und zusätzlich personell gut ausgestattet sind, integrierte die Betriebsleitung umfassende Aufgaben der schlosserischen Instandhaltung und des Labors. Im Rahmen des Projekts werden diese Aufgaben dann noch einmal um einfachere PLT-Tätigkeiten und Laborarbeiten erweitert, so dass die Aufgabenspanne der Beschäftigten noch einmal größer wird. Zugleich wurde die schlosserische Instandhaltung um zwei Handwerker reduziert, im Labor wurden vier Stellen abgebaut mit der Konsequenz, dass die ohnehin nur noch selten laufenden verfahrenstechnische Versuche nicht mehr im Betrieb stattfinden.

Beide Pilotbetriebe sind durch eine Ausgangssituation charakterisiert, die eine sehr geringe Innovationsaffinität aufweist. Die interne Rationalisierungsoption der Prozessoptimierung gilt als ausgereizt, gleichzeitig stellt der Normallauf hohe Anforderungen an die Regulierungstätigkeiten. Darüber hinaus ist die Marktposition eher geschwächt, bei der unternehmensinternen Bereinigung des Produktportfolios sind die Betriebe nur knapp und unter erheblichen Rationalisierungsaktivitäten der Schließung entgangen.

Eine weitreichende Umsetzung des Projekts ist damit in mehrfacher Hinsicht problematisch und erstens für die Aufgaben- und Funktionserweiterung folgenreich. Sowohl in KS als auch in PF sind umfassende Rationalisierungsbemühungen und Umstrukturierungen unmittelbar vor Start des Pilotversuchs durchgeführt worden. Aufgabenintegration und die Erhöhung der Einsatzflexibilität der Beschäftigten sind sehr weitreichend umgesetzt, und so überrascht es wenig, dass die Schichten nur sehr eingeschränkte Arbeitsveränderungen feststellen, die sich explizit mit der Einführung von Teamarbeit in Verbindung bringen lassen. Zusätzlich wirken sich technische bzw. chemiespezifische Bedingungsfaktoren in beiden Fällen hemmend auf die nun in Eigenregie zu planende Realisierung von Rotationsregelungen aus. Vor allem in der Komplexität der Anlagen sehen die Beschäftigten ein gewichtiges Hindernis für eine umfassende horizontale Aufgabenintegration und schätzen sehr realistisch ein, inwiefern eine Rotation möglich bzw. überhaupt sinnvoll für den Prozess insgesamt ist. 
„Es kann funktionieren, sicher, nur, es gibt Anlagen, die sind sehr komplex, andere, die sind ziemlich einfach. Das heißt, es ist fast nicht möglich, an allen Anlagen gleich stark zu sein. Es wird immer eine Anlage geben, wo ich, wo alle ihre Schwierigkeiten haben.“ (Beschäftigter PF/3)

Darüber hinaus sind die Beschäftigten insbesondere im Fall KS den chemiespezifischen Dokumentations- und Sicherheitsvorschriften verpflichtet, die für die qualifikatorischen Voraussetzungen der einzelnen Prozesse stark formalisiert und umfassend sind. So definieren etwa die Good-Manufacturing-Practices (GMP) detailliert, wie lange die Beschäftigten an einem Anlagenteil angelernt werden müssen, um eine bestimmte Qualifikationsstufe erreicht zu haben. Erst auf Stufe drei kann dann selbständig, d.h. ohne die aktive Mithilfe (Stufe eins) oder die Überwachung durch einen erfahrenen Kollegen (Stufe zwei), ein Anlagenteil betreut werden. Wird in einem Zeitraum von sechs Monaten nicht mehr an der Anlage gearbeitet, verliert der Beschäftigte automatisch eine Qualifikationsstufe. Er darf dann nicht mehr selbständig den Prozess überwachen, sondern muss mit einem Kollegen, der auf Stufe drei ist, zusammen arbeiten. Im Arbeitsalltag den Überblick über die komplizierten Regelungen zu behalten, ist nach Aussagen der Beschäftigten nicht immer leicht und folgenreich für die Rotationspläne:

„Wir haben die Arbeitsplätze einfach nicht oft genug gewechselt. Das ist eben so eine Sache. Selbst wenn ich meine, ich war da vor geraumer Zeit und der Schichtmeister kommt und fragt, dann müsste ich erst mal nachschauen, wann das war, so lange ist das her.“ (Beschäftigter KS/2)

Zweitens hat sich auch in den von uns untersuchten Betrieben die Arbeit durch den erheblichen Personalabbau verdichtet. ${ }^{166}$ Zeitdruck und Stress gehören für die meisten Beschäftigten zum Arbeitsalltag dazu, so dass die schichtinternen Anlernprozesse immer wieder von betrieblichen Anforderungen durchkreuzt werden. Dabei hat der fehlerfreie Ablauf der Produktion erste Priorität. In den ohnehin schon dünn besetzten Schichtmannschaften fehlen deshalb häufig die Ressourcen zum Anlernen von Kollegen.

„Da frage ich nur, wie soll das denn gehen? Wenn da noch jemand krank ist, da renne ich durch die Anlagen, da kann ich nicht noch dem Kollegen erklären, was da läuft.“ (Beschäftigter $\mathrm{PF} / 2$ )

Hier knüpft das Konzept an Bedingungen an, die sich aus traditionellen Rationalisierungseffekten der Vergangenheit begründen und die zum manifesten Umsetzungshindernis werden können. „Mehr wie laufen kann man nicht!“ ist eine häufig wiederkehrende Aussage in den Interviews.

Als dritte wichtige Konsequenz ist festzuhalten, dass auch die Potentiale zur Neudefinition der „Schichtmeister-Rolle“ erheblich eingeschränkt sind: Innovationshandeln bleibt eindeutig jenseits der „Kragenlinie“ zentriert. Die Schichtmeister sind weitgehend von betrieblicher Planung, einer schichtübergreifenden Kostenkontrolle und koordinierenden Aufgaben ausgeschlossen und bleiben ihrer traditionellen „Schichtführer-Rolle“ verhaftet.

\footnotetext{
${ }^{166}$ Es handelt sich hier in der Regel nicht um Entlassungen, sondern um altersbedingtes Ausscheiden von Kollegen, die dann nicht ersetzt wurden.
} 
Das hierarchische Muster der kommunikativ-kooperativen Strukturen des tradierten Sozialgefüges ist weitgehend stabil.

Dies wirkt sich negativ auf das Gestaltungsziel der gruppengetragenen Selbstorganisation aus. Das Selbstverständnis der Schichten basiert nach wie vor auf der Vorstellung von einer homogenen Gruppe - jedenfalls unter dem Aspekt der sozialen Kohäsion. Beispielhaft dafür steht die Diskussion um die Frage, ob der Schichtmeister mit zum Team gehören soll. Recht eindeutig sind die Schichten der Auffassung, dass der Meister als Teammitglied zu definieren sei. Freilich, nicht aus der Überzeugung heraus, dass man als Arbeiter ohne Vorgesetzten ohnehin nichts tun dürfe, sondern vielmehr aus dem gewachsenen Gruppenbewusstsein heraus. Der Schichtmeister gehört, ausgehend von der betrieblichen Realität, zur Arbeitsgruppe dazu, denn er ist zwar Vorgesetzter, aber eben auch ein Kollege, der mitarbeitet wie jeder andere - eine Tatsache, die auch durch das neue Konzept nicht verändert wird. Einige Interviewte heben diesen kollegialen Aspekt sehr positiv hervor. Bestrebungen, den Meister aus den Teams heraus zu holen, verstehen sie als „Keil“, der seitens des Betriebs in die Schichten getrieben wird.

In der Dimension der gruppengetragenen Selbstorganisation zeigt sich folgerichtig eine nur gering ausgeprägte Motivation seitens der Beschäftigten. Beispielhaft dafür steht die Wahl des Teamsprechers. Im überwiegenden Teil der Schichten wird der stellvertretende Schichtmeister zum Teamsprecher gewählt. ${ }^{167}$ Dezidiert wird an alte Kommunikationsund Kooperationsstrukturen angeknüpft:

„Da haben sich alle geduckt und einer hat auf mich gezeigt und da war ich dann auf einmal einstimmig gewählt. Da hieß es dann, du redst doch immer mit denen da oben, du kannst das doch."(Beschäftigter PF/3)

Der zusätzliche Nutzen eines Teamsprechers, der zwischen Management und Schicht vermitteln soll, wird nicht wirklich gesehen. Dazu hatte und hat man auf Schicht die gewachsene Sozialstruktur, eine Veränderung gilt als nicht notwendige Störung.

Die Teamsitzungen laufen eher schleppend, das Gros der Befragten gibt zu verstehen, dass diese Art der Formalisierung in ihren Augen „unsinnig“ sei. Die Schichten würden sich ohnehin ständig in der Messwarte und im Pausenraum treffen, da könne alles Wesentliche besprochen werden. Die durch das Projekt in die Schichten getragenen Themen sind denn auch schnell erschöpft. Während sich die Teams in den ersten Sitzungen mit der Diskussion von Regeln hinsichtlich Urlaubs-, Freischicht- und Qualifizierungsplanung auseinandersetzen, gelingt der Übergang von der Bearbeitung gruppeninterner Themen zu betrieblichen Fachthemen von den Mannschaften nicht - und kann in Anbetracht fehlender Impulse auch nicht gelingen. So herrscht in den Schichtmannschaften zu recht eine gewisse Ratlosigkeit, wie es weitergehen solle „mit der Teamarbeit“.

\footnotetext{
${ }^{167}$ Ein aus den Fertigungsindustrien unbekanntes Phänomen. Weder Vorarbeiter noch stellvertretende Schichtführer stehen hier als Kandidaten für die Belegschaften zur Debatte. Wir werden im Zusammenhang mit den Funktionen des Meisters noch darauf zu sprechen kommen. Während in der traditionellen Arbeitsorganisation in den Fertigungsindustrien die Meister ihre „Eckkräfte“ (Mühlbradt, Krings 1996) selektieren und damit eine erhebliche Macht inne haben, spielt dies in der Chemischen Industrie nicht die herausragende Rolle.
} 
„Von denen da oben, da kommt jetzt nichts mehr. Wir haben schon zwei, drei Monate keine Sitzung mehr gemacht, weil die Jungs und ich wissen gar nicht mehr, womit wir uns auseinandersetzen sollen.“ (Beschäftigter KS/2)

Aussagen, die in diese Richtung gehen, unterstreichen, dass die Beschäftigten nicht befürchten, „mühsam aufgebautes informelles Arbeitsverhalten, verdeckte Spielräume und leistungsrelevante Reserven“ (Schumann 1993) zu verlieren, und daher einen „arbeitspolitischen Konservatismus“ an den Tag legen. Skeptische Zurückhaltung der Beschäftigten oder „strukturelles Beharrungsvermögen“ sind die Ausnahme. Viel eher verdeutlicht die abwartende Haltung der Teams, dass aus ihrer Sicht keine besonders „dringlichen“ Themen zu besprechen wären und die Prozesse kaum Potential und Realisierungsmöglichkeiten für Optimierungsvorschläge der Beschäftigten aufweisen.

Dies entspricht auch der Sicht des betrieblichen Managements: Im Normalbetrieb der Anlagen werden seitens des Betriebsleiters wie auch der Betriebsingenieure keine Potentiale mehr gesehen, die durch eine stärkere Einbindung des Know-Hows der Beschäftigten auszuschöpfen wären. Die Produkte sind lange am Markt, sowohl qualitative wie quantitative Verbesserungen tragen nicht zu einer erhöhten Performance bei. Zugleich bindet der Normalbetrieb die Beschäftigten aber auch so intensiv, dass diese für aufwändigere verfahrenstechnische Modernisierungen nicht mehr „freigespielt“ werden können. Im Fall PF ist dies dem Personalabbau geschuldet, im Betrieb KS den Sicherheitsbestimmungen.

Dies hat erhebliche Konsequenzen für die Dynamik des KVP, der in diesen Fällen auf die kleinschrittige Verbesserung reduziert wird. Aus Perspektive des Managements muss die Initiative zum KVP von den Teams ausgehen. Weder werden Themen es aus der Betriebsleitung vorgegeben, noch Vereinbarungen über zu bearbeitende Themenkomplexe geschlossen. Die Bearbeitung Team-übergreifender Probleme oder die Diskussion komplexer Fragestellungen in fach- und hierarchieübergreifenden Workshops findet nicht statt.

Verstärkt wird diese Situation durch die Haltung des Managements zu den Optimierungsvorschlägen, die im Rahmen des KVP eingereicht werden. Diese gelten eher als „Peanuts“ und „Kinkerlitzchen“. Ihre Bearbeitung wird sehr deutlich als Zeitverschwendung bezeichnet. Dass diese Einstellung kein Geheimnis für die Schichtmannschaften ist und der gruppengetragenen Prozessoptimierung entgegenwirkt, wird vor allem in den Gruppendiskussionen deutlich, in denen die fehlende Anerkennung durch die „Experten“ moniert wird. Diese seien nach wie vor nicht bereit ,sich mal auf eine Stufe mit den Schichten zu stellen“. Stellvertretend für viele meint ein Beschäftigter:

„Ich habe manchmal den Eindruck, die denken sich: Wie kann man sich erdreisten als normaler Malocher sich einzumischen in Sachen, die doch nur die Obersten können. Alles, was nicht auf deren Mist gewachsen ist, ist schlecht.“ (Beschäftigter KS/3)

Insbesondere die Ingenieure geraten in die Kritik. Die Beschäftigten unterstellen ihnen, dass sie systematisch Vorschläge ignorieren bzw. aus Kostengründen ablehnen. Ein Argument, dass den wenigsten einleuchtet: 
„Das ist doch lächerlich: Mancher Vorschlag kostet wirklich nicht viel, würde aber Kosten einsparen, der wird glatt abgelehnt. Gleichzeitig gibt es aber Anlagenteile für zig Millionen Mark, wo man weiß, das funktioniert nicht. (...) Das ist eben das Seltsame: Wenn die Ingenieure was berechnet haben, wird es immer durchgesetzt. Wenn wir einen Vorschlag machen, dann wird der abgelehnt.“ (Beschäftigter PF/2)

Dass die Beschäftigten die Qualität ihrer Vorschläge anders wahrnehmen, als das Management, erklärt sich nur teilweise aus den unterschiedlichen Vorverständnis der Prozesse bzw. differenten Wissensbeständen. Richtig ist, dass ein kostensparender Vorschlag in der betriebsübergreifenden Perspektive an einer anderen Stelle der Wertschöpfungskette ein nicht unerhebliches Mehr an Kosten verursachen kann, oder dass schlicht die betrieblichen Ressourcen zur Umsetzung guter Verbesserungs- und Optimierungsansätze fehlen. $\mathrm{Zu}$ einem Problem gerät dies in den untersuchten Fällen, weil nicht offen über die komplizierten Zusammenhänge kommuniziert wird. Die Gruppen werden vom Management im alten Anweisungsstil nur ,in Kenntnis“ gesetzt, dass ein Vorschlag nicht angenommen ist, eine fach- und sachgerechte Begründung hingegen bleibt oft im Tagesgeschäft auf der Strecke. Von einzelnen Beschäftigten wird dies denn auch zugespitzt als „Schlüsselenttäuschung“ im Zusammenhang mit der Einführung von Gruppenarbeit bewertet. Darüber hinaus hat in den Mannschaften der massive Personalabbau der letzten Jahre seine Wirkungen hinterlassen. Diese äußern sich in einer latenten Unsicherheit und Angst in Bezug auf den eigenen Arbeitsplatz.

„Zwölf Leute haben die entlassen und niemand Neues ist gekommen. Ist doch klar, dass man da Angst hat.“ (Beschäftigter PF/1)

Gleichzeitig wirken diese Rationalisierungserfahrungen auch auf die eigene Beteiligung am KVP. Die Beschäftigten sind skeptisch, inwiefern man auch eigenständig Vorschläge zur Prozess- und Anlagenoptimierung machen soll. Die rationalisierungswirksame Kehrseite der Beteiligung an Optimierung ist immer präsent:

„Wir haben schon Gruppenvorschläge gehabt, die sind wunderbar. Aber irgendwann kommt man in Bereiche, da hat man 15 Minuten hier, 20 Minuten da eingespart. Und dann hat man sechs Stunden voll und dann heißt es: Ihr seid 13 Mann, da kommen wir doch jetzt auch mit 12 aus. Dem Prozess ist das egal, der funktioniert dann ja weiter. Aber wer geht dann?“ (Beschäftigter PF/5)

Der durchaus positiven Bewertung der neuen Möglichkeiten im Optimierungsprozess seitens der Beschäftigten stehen auf der Ebene der betrieblichen Praxis reale Grenzen gegenüber. Von den Teams wird dies als enttäuschte Erwartung an eine stärkere Einbindung in das Innovations- und Optimierungshandeln, die sie mit dem Konzept verbunden haben, beschrieben.

Damit ist für die beiden Betriebe eine Situation beschrieben, wie sie im Trendreport Rationalisierung als traditionell-hierarchischer Organisationstyp definiert wird, allerdings auf einem deutlichen höheren Anforderungsniveau für die Beschäftigten. Bedingt durch den massiven Personalabbau in der Vergangenheit gehören eine hohe Einsatzflexibilität, Rotation sowie eine enge Kooperation der Schichten mit den Handwerkern inzwischen zum Arbeitsalltag. Arbeitseinsatz- und Arbeitsteilungspraxis haben sich sicher nicht aufgelöst, aber doch zumindest gewandelt. Das Modernisierungsproblem wird jedoch nach wie vor 
auf dem Boden der etablierten Strukturen in Angriff genommen, die Beschäftigten bleiben vom eigentlichen Innovationsgeschehen ausgeschlossen. Dies ist im wesentlichen auf den Reifegrad der Produkte sowie den damit einhergehenden Technisierungsgrad der Anlagen zurückzuführen. Diese lassen nur eine gebremste Innovationsdynamik zu und sind bezogen auf erweiterte Spielräume für Planungs-, Steuerungs- und Optimierungshandeln der Beschäftigten unterschüssig. So überwiegt in den untersuchten Betrieben die etablierte innerbetriebliche Organisationsstruktur, die durch die abwehrende Haltung der Betriebsleitung noch unterstützt wird. Im Ausspruch eines Betriebsmeisters - „Wir sind schon gut, wir können nur noch besser werden“ - kommt diese das Projekt zusätzlich hemmende Einstellung zum Tragen. Die Probleme, die aus Sicht des betrieblichen Managements vor Ort durchaus existieren, sind von daher aus ihrer Perspektive mit dem Gestaltungsansatz nicht zu lösen. Die Grundidee des Projekts ist damit nicht verspielt: Von den Beschäftigten wird das Konzept insgesamt befürwortet, die Umsetzung hingegen als mangelhaft eingeschätzt und negative Folgen, allen voran die verschärfte Belastungssituation, betont.

\subsubsection{Neue Formen der Arbeitsorganisation bei starker Innovationsaffinität}

In den Betrieben AI und PH wird selbstorganisierte Gruppenarbeit auf hohem Realisierungsniveau umgesetzt. Beide Fälle zeichnen sich dadurch aus, dass sie erstens in ein hoch dynamisches Umfeld eingebunden sind. Die Betriebleitung ist eng in betriebsübergreifende Innovationsprojekte eingebunden. Zweitens werden in AI und PH Produkte hergestellt, denen wegen ihrer außerordentlichen Marktperformanz im Rahmen des Portfoliomanagements auch zukünftig eine hohe Bedeutung beigemessen wird. Diese Produkte sind drittens sowohl im Hinblick auf ihren Reifegrad wie die Verfahrenstechnik noch nicht ausgereift, und eine sukzessive Optimierung verbunden mit umfassenden empirischen Testphasen bestimmt den Normalbetrieb der Anlagen, so dass es auf dem Shop floor zu einer Überlappung von Innovations- und Produktionsgeschehen kommt.

Der Betrieb PH gehört zum Arbeitsgebiet Gesundheit, das sich seit Ende der 1980er Jahre zum einträglichsten der sechs Unternehmensbereiche entwickelte. So wuchs etwa der Anteil am operativen Ergebnis von 23 Prozent in 1988 auf 51 Prozent in 1994. Selbst in den Krisenjahren 1992 und 1993 konnte die Umsatzrentabilität gesteigert werden. In den Geschäftsbereich Pharma fließt der größte Anteil der FuE-Ausgaben des Unternehmens. Im Zentrum der strategischen Ausrichtung geht es dabei vor allem darum, die Entwicklung und Produktion von Blockbustern für ausgewählte Märkte voranzutreiben. Im Betrieb selbst wurde 1990 eine veraltete Anlage komplett durch modernstes technisches Know-how ersetzt, um einen solchen hochinnovativen und gewinnträchtigen Wirkstoff herzustellen. Wie von den Marketingexperten vorausgesehen, entwickelte sich das neue Produkt tatsächlich zu einem von zwei Standbeinen im Bereich Pharma. Der Markt gilt im Hinblick auf die absetzbaren Mengen als kaum zu sättigen. Der Betrieb erfährt aus diesem Grund eine hohe Aufmerksamkeit, denn er verspricht, nicht nur die FuE-Kosten relativ schnell zu erwirtschaften, sondern darüber hinaus auch bis zum Auslaufen des Patentschutzes einen satten Überschuss zu erzielen.

Diese herausragende Stellung im Portfolio des Unternehmens wirkt sich einmal auf die Anbindung an die Servicebereiche aus. Im Gegensatz zu anderen Betrieben werden Reparaturen prioritär behandelt, da jede Minute Produktionsstillstand unbedingt vermieden werden soll. Darüber hinaus überlappen sich auch Innovations- und Produktionshandeln 
in besonderer Weise. Wegen der hohen Gewinnerwartungen stand in der Anlaufphase die Inbetriebnahme im Vordergrund, um das Produkt möglichst schnell auf den Markt zu bringen. Sowohl der Reifegrad der Anlagen selbst wie auch die Ausbringungsleistungen stehen von daher auch im Untersuchungszeitraum im Fokus des Optimierungshandelns. Für die Betriebsleitung steht damit einmal die Weiterentwicklung von Produkt und Produktionsverfahren in enger Zusammenarbeit mit Forschung, Entwicklung und Ingenieurtechnik auf der Agenda. Darüber hinaus wird eine kontinuierliche Erhöhung der ausgebrachten Produktmengen angestrebt, um so den Preis pro Tonne zu senken und auch nach Ablauf des Patentschutzes die Marktführerschaft zu sichern. Die technische Anlagenverfügbarkeit, ein störungsfreier Betrieb sowie eine konstante Produktqualität gelten als wichtige Stellgrößen im Rahmen dieses Prozesses.

Traditionelle Rationalisierungsinstrumente wie Arbeitsverdichtung und Personalreduktion greifen unter dieser Perspektive zu kurz, denn gerade im Bereich Pharma haben die Personalkosten nur eine relativ geringe, die besonderen Kompetenzen der betrieblichen Akteure dafür eine umso größere Bedeutung. Einmal werden sie zur Optimierung aktueller betrieblicher Abläufe gebraucht, darüber hinaus gelten sie als Ressource zur Inbetriebnahme von Produktionsanlagen, die ähnliche Wirkstoffe herstellen sollen.

Beispielhaft dafür ist der in unserem Untersuchungszeitraum stattfindende Wechsel des Betriebleiters an einen deutschen Nachbarstandort. Ein Nachfolgeprodukt des im Betrieb PH produzierten Wirkstoffes sollte zunächst in den USA hergestellt werden. Dort gelang es aber weder, genügend kompetente Mitarbeiter zu finden, noch das Know-how zu transferieren. Im Unternehmen beschloss man deshalb, den Betrieb am deutschen Nachbarstandort aufzubauen. Erneut schlug der Transfer fehl. Schließlich wurde das Problem durch den sukzessiven Abzug kompetenter Akteure aus PH in die neue Produktionsanlage bewältigt. Knapp drei Jahre nach unserer Recherche haben neben dem Betriebsleiter ein Viertel der Beschäftigten sowie zwei Schichtmeister in den neuen Betrieb gewechselt. In diesem Beispiel spiegelt sich ein wesentlicher Teil der Geschäftsbereichskultur Pharma wider, die traditionell sowohl in Forschung und Entwicklung als auch in Produktion und Fertigung auf eine langfristig aufgebaute, hochspezifische und konzentrierte kollektive Beschäftigtenkompetenz zur Bewältigung der spezifischen Anforderungen setzt - bzw. setzen muss.

Der Betrieb AI gehört zum Geschäftsbereich Anorganische Industrieprodukte im Arbeitsgebiet Industrieprodukte, dass seit Ende der 1980er Jahre durch sinkende Umsätze und eine damit einhergehende Bereinigung des Produktportfolios gekennzeichnet ist. Der Bereich entstand dabei erst 1994 aus der Zusammenlegung der Bereiche Anorganische Chemikalien und Pigmente, Keramik. Der Forschungsschwerpunkt liegt inzwischen auf der Spezialchemie, für die der Bereich die Produkttechnologieführerschaft anstrebt. Das Unternehmen hat in Anbetracht der Konjunkturflaute im Baugewerbe und der starken osteuropäischen Konkurrenz hier in besonderer Weise eine Fokussierung auf die internen Kernkompetenzen vorgenommen. Viele Betriebe wurden geschlossen oder verkauft, so dass inzwischen auf dem Gelände des Stammwerks ausgegliederte Produktionsstätten mit fremden Firmennamen zu finden sind. Der Betrieb selbst steht mit Umsatzrenditen, die denen im Bereich Pharma ähneln (zwischen 10 und 20 Prozent), als einer der wenigsten im Geschäftsbereich glänzend da. Maßgeblich für das positive operative Geschäft ist ein Produkt, mit dem sich der Betrieb als Marktführer positionieren kann. Um diese Position beizubehalten, ist der Betrieb stark in Verfahrensentwicklungen eingebunden. Das Gros der Versuche läuft während der Tagschicht. Insgesamt werden im Betrieb drei Hauptpro- 
dukte in unterschiedlichen Varianten gefahren. Die Anbindung an die Kunden ist sehr eng, da flexibel auf Kundenwünsche reagiert werden muss. Die Einhaltung sowohl der Lieferzeiten wie der konstanten Produktqualität haben Priorität, zugleich ist der Kostendruck durch die starke Konkurrenz hoch.

Die Betriebsleitung besteht aus Kostengründen nur noch aus einer Person, die die Funktionen des Betriebsingenieurs, des Betriebs- sowie des Werkstattleiters in sich vereinigt. Die organisatorische Anbindung der indirekten Bereich ist aufgrund dieser personellen Konstellation gut und relativ konfliktfrei. Regelmäßige Gespräche und Informationsveranstaltungen zwischen den Schichtmannschaften und den Handwerkern bzw. dem Labor sowie umfassende Zusatzqualifizierungen (Labor-, PLT- und Schlosser-Tätigkeiten) für die Beschäftigten forcieren die enge Zusammenarbeit.

Der Betrieb sieht sich insgesamt mit einer Markt- und Produktstruktur konfrontiert, die ein erhebliches Maß an Flexibilität und Reagibilität erfordert und zugleich zur Sicherung der Wirtschaftlichkeit des Betriebs innovatorisches Handeln voraussetzt. Vor dem Hintergrund der daraus resultierenden angespannten Situation setzt der Betriebsleiter auf die Kompetenzen aller betrieblichen Akteure und sucht selbst aktiv nach Einbindungsmöglichkeiten der Schichtmannschaften in betriebliche Optimierungsaktivitäten.

Beispielhaft dafür steht etwa die im Betrieb sehr häufig anstehende Reparatur der störanfälligen Pumpen. Diese durften aus rechtlichen Gründen nicht im Betrieb repariert werden, sondern mussten in die Zentralwerkstatt. Eine für die Produktion belastende Situation., da in dieser Zeit nicht produziert werden konnte. Da die Lieferfristen insgesamt sehr eng gesetzt sind und Liefertreue für die Kunden ein wichtiger Aspekt bei der Auswahl der Lieferanten ist, konnten Terminverzögerungen immer den Verlust eines Kunden und damit sicherer Umsätze bedeuten. Darüber hinaus musste für die Vergabe der Arbeit ein hoher Betrag an die Zentralwerkstatt gezahlt werden. Der Betriebsleiter hat aus diesem Grund zunächst sehr genau geprüft, inwiefern rechtliche Bestimmungen tatsächlich den Einsatz der Zentralwerkstatt erfordern, oder ob diese Arbeiten nicht von Betriebshandwerkern und Schichtmannschaften erledigt werden können. Diese von ihm gegen viele Widerstände im Unternehmen durchgesetzte Regelung spart dem Betrieb hohe Kosten und stellt eine neue, fachübergreifende Anforderung an die Beschäftigten.

Als zusätzliche Herausforderung sieht sich die Betriebsleitung mit der Übernahme einer Nachbaranlage konfrontiert. Die Anlage insgesamt ist zu optimieren, um eine wirtschaftliche Produktion zu gewährleisten, sowie die Qualifizierungsmaßnahmen für die Schichtmannschaften für die neue Anlage bei ohnehin knapper personeller Ausstattung zu organisieren.

Kennzeichnend für diese betrieblichen Konstellationen ist eine hohe Innovationsaffinität, die sich aus den Markt-, Produkt- und Innovationsanforderungen ergibt. Das betriebliche Management setzt zur Bewältigung dieser Aufgaben aktiv auf die Kompetenz der Schichtmannschaften. Das arbeitspolitische Gestaltungsprojekt wird von der Betriebsleitung als Chance begriffen, die daraus resultierenden Probleme adäquat zu bearbeiten. ${ }^{168}$

Auf dem Shop floor ist bezogen auf die Aufgaben und Funktionsintegration der Beschäftigten festzustellen, dass sich der Umfang der unmittelbaren Produktionsaufgaben der

\footnotetext{
${ }^{168}$ Die folgenden Ergebnisse basieren auf einer Intensivrecherche von je zwei Wochen in den Betrieben AI und PF, in denen 19 Expertengespräche, 21 Beschäftigteninterviews, 112 standardisierte Beobachtungen sowie 13 Arbeitsplatzbeobachtungen durchgeführt wurden.
} 
Beschäftigten deutlich erweitert hat: Die Betriebe haben systematisch eine breite Aufgaben- und Funktionsintegration vorangetrieben, die auf eine Ausweitung der Prozessarbeiten (funktionsorientierte Arbeiten) zielt. Dazu entwickelten betriebliches Management (in der Regel die Betriebsmeister) zusammen mit den Teams in den Betrieben Tabellen, in der alle von den Schichtmannschaften bereits erfüllten Aufgaben eingetragen werden. ${ }^{169}$ Auf dieser Grundlage erstellten die Teams eine Qualifikationsmatrix, auf der die von jedem Teammitglied bearbeiteten Aufgaben und Verantwortlichkeiten abzulesen sind. Aus dieser Liste entstand schließlich die Qualifizierungsmatrix, die den Soll-Zustand pro Beschäftigten im Team angibt. In diese werden in die Teams hinein verlagerte neue Anforderungen aufgenommen, beispielsweise die Bestellung neuer Einsatzprodukte. Die Teams entscheiden eigenständig darüber, wie sie den Soll-Zustand, d.h. die Qualifizierung aller für möglichst alle Aufgaben sicherstellen. Ziel ist es, eine hohe Einsatzflexibilität, größere Arbeitsumfänge und Rotation über alle Arbeitsplätze langfristig zu gewährleisten. Ferner wird die Integration von Umfeldaufgaben verstärkt, und schlosserische sowie elektrotechnische Instandhaltungsarbeiten, also indirekte, „berufsübergreifende“ Arbeiten sind in die Teams integriert worden. Je nach betrieblicher Ausgangssituation wurden Zusatzqualifikationen im Bereich Labor, PLT und Schlosser sukzessive für alle Teammitglieder durchgeführt sowie regelmäßige Schulungsmaßnahmen angeboten. Seitens des betrieblichen Managementes wurde etwa in einem Fall in Kooperation mit den Schichten ein systematisches Qualifizierungsprogramm, das sowohl die Fähigkeiten der Schichtmannschaften zur Laboranalyse erhöht (bis zu zehn Stunden Einzelunterricht beim Laborleiter), ein Kursprogramm für die einfacheren Schlossertätigkeiten umfasst und ein Aufqualifizierungsprogramm für einfache PLT-Arbeiten vorsieht, ausgearbeitet. Zwar ist zu berücksichtigen, dass eine Reihe von schlosserischen Instandhaltungsarbeiten immer schon beim Team lagen, hier also lediglich eine Offizialisierung vorgenommen wurde. Dennoch hat, und dies gilt insbesondere für die Übernahme der Labortätigkeiten, eine beachtliche Aufgabenerweiterung stattgefunden. In den Gruppengesprächen mit den Mannschaften wird sehr deutlich, dass die Beschäftigten diese Maßnahmen durchaus in der Ambivalenz zwischen Arbeitsverdichtung und dem positivem Moment von Aufgabenerweiterung, Höherqualifizierung und wachsender Interessantheit der Arbeit sehen. Von Seiten der Instandhaltungsingenieure wie auch der Betriebsleitung wird betont, dass durch das gewachsene Qualifikationsniveau im schlosserischen und im Laborbereich eine bessere Anlagenbetreuung und erhöhte Anlagenverfügbarkeit erreicht wird. Den Mannschaften wird zugebilligt, dass sie die Arbeit an den Anlagen nun differenzierter und sensibler ausführen und proaktive Maßnahmen im Sinne von vorbeugender Instandhaltung wesentlich eher realisieren können.

Um den gesetzlichen Auflagen zu entsprechen, sind die Eingriffsbefugnisse in sogenannte Positivlisten detailliert festgelegt. Zusätzlich müssen die im Betrieb von den Beschäftigten durchzuführenden Instandhaltungsarbeiten (Instandsetzung, Wartung/Inspektion) folgende Merkmale aufweisen:

- Sie müssen unvorhergesehen sein (mit Ausnahme von Wartungs- und Inspektionsarbeiten)

- Sie überschreiten einen bestimmten Zeitaufwand nicht

\footnotetext{
${ }^{169}$ In den Betrieben werden dann einzelne Rubriken gebildet, in denen die Teilschritte abzulesen sind. So setzt sich etwa „Messwarte: bedienen“ zusammen aus den Teilaufgaben „Kolonne fahren; Anlage über Bildschirm fahren; Anlage über Tafeln fahren; Telefonate entgegennehmen und weiterleiten; Schreiberrollen und Farbe wechseln; Temperaturen und Druckverhältnisse einstellen; Getränkeverkauf; Reinigung der Messwarte“.
} 
- Sie erfordern keine Spezialgeräte

- Es ist kein Spezialgewerk erforderlich ( Schlosser oder Elektriker)

Insgesamt haben die Betriebe auf diesem Weg eine Integration von Umfeldaufgaben für die Gruppen sichergestellt.

Eng damit verbunden ist die Ausprägung der gruppengetragenen Selbstorganisation. In beiden Fällen gilt die in den Teams systematisch verankerte Selbstorganisation ${ }^{170}$ als wesentlicher Bestandteil des Projekts und ist ein wichtiges Unterscheidungskriterium zu den „strukturkonservativen“ Modellen der Betriebe KS und PF. In den Betrieben sind Gruppengespräche wie Wahlen von Gruppensprechern durch die Beschäftigten institutionell verankert. Die Teamgespräche finden regelmäßig statt und werden von den Beschäftigten selbständig gestaltet. Dispositive Aufgaben wie Urlaubs-, Personaleinsatz-, Freischichtund Qualifizierungsplanung fallen in den Entscheidungsbereich der Gruppen und werden in den Gruppengesprächen koordiniert. Die Gruppen müssen sich dabei an die betrieblichen Regeln, die den einzelnen Planungsbereichen zugrunde liegen, halten. Vom Einfluss der Vorgesetzten sind sie dabei weitgehend unabhängig. ${ }^{171}$ Schließlich werden in den Teams bestimmte Regeln für im Prozess der Selbstorganisation anstehende Aushandlungen verbindlich. Diese orientieren sich an Grundprinzipien wie dem Prinzip der Gleichberechtigung (Vermeidung von Diskriminierung und Ausgrenzung), dem Prinzip demokratischer Entscheidung (nicht das lauteste, sondern das überzeugendste Argument setzt sich durch), sowie der Einhaltung gruppeninterner Vereinbarungen wie auch betrieblicher Vorgaben. Um ein Höchstmaß an gruppeninterner Gerechtigkeit etwa bei der Frage, welches Teammitglied wann und wie lange in Urlaub geht, zu erreichen, haben die Gruppen interne Abstimmungsmodi entwickelt. Wie aus gruppensoziologischer Perspektive zu erwarten, ${ }^{172}$ sind diese Gruppenbildungsprozesse insgesamt nicht konflikt- und krisenfrei, wie die Äußerung eines Teamsprechers stellvertretend für andere belegt:

„Ich würde gern haben, dass jeder zu jedem anderen hält. Dass nicht so sehr rumgespottet wird nach dem Motto, du bist Jugoslawe oder du hast nur Lohngruppe 3. (...) Es wird zwar schon mehr untereinander geholfen. Aber auf der anderen Seite wird jetzt auch gesagt, wenn einer irgend was gemacht hat: das hast du selbst gemacht, das musst du jetzt auch auslöffeln. Früher hat man da nur auf den Schichtmeister geschimpft. Jetzt heißt es auch öfter: Du bist selber schuld. Das liegt daran, weil die intere Reibung jetzt da ist.“ (PH/3)

Diese „Zumutungen der Selbstregulation“ (Minssen 1999) werden allerdings von den Beschäftigten in der Regel ohne externe Intervention eigenständig geregelt. Der Gruppensprecher funktioniert in diesen Fällen als Koordinator und besitzt, meist forciert durch die Teilnahme an Gruppensprecherschulungen, eine hohe Kompetenz in Moderationstechniken und Konfliktmanagement. Der Sprecher bleibt jedoch in jedem Fall primus inter pares, ist nicht weisungsbefugt und vollständig in den Arbeitsprozess integriert.

\footnotetext{
${ }^{170}$ Der Begriff der Selbstorganisation bezieht sich im folgenden auf die von den Gruppen zu erbringenden organisatorischen Leistungen.

${ }^{171}$ Eine solche Regelung stellt etwa im Hinblick auf die Urlaubsplanung die festgesetzte Mindestbesetzung des Betriebes dar, für den Personaleinsatz ist der Grad der Qualifikation der Beschäftigten für die einzelnen Arbeitsplätze zu berücksichtigen.

${ }^{172}$ Vgl dazu bspw. den von Fröhlich schon Anfang der 1980er Jahre veröffentlichten Text „Machtprobleme in teilautonomen Arbeitsgruppen“ (1983).
} 
Die Einbindung der Beschäftigten in das Innovationsgeschehen wird einmal über die Definition von Prozessoptimierung als Teamaufgabe gewährleistet. Parallel zum auf individueller Initiative basierenden betrieblichen Vorschlagswesen sind die Gruppen aufgefordert, zum kontinuierlichen Verbesserungsprozess beizutragen. Dies ist vom betrieblichen Management ausdrücklich nicht allein in der Perspektive der Optimierung betrieblicher Abläufe an die Teams herangetragen. Im Gegenteil, auch die Verbesserung der Arbeitsbedingungen gilt als Ziel der Prozessoptimierung. Dies sichert dem Management eine hohe Unterstützung bei Optimierungsprojekten. Im Unterschied zu den Betrieben KS und PF wird die Beteiligung am KVP von den Teams auch mehrheitlich positiv bewertet. Zwar wird von den Beschäftigten gerade in Anbetracht der allgemeinen ökonomischen Situation des Unternehmens die Rationalisierungsbeteiligung durchaus nach wie vor skeptisch beurteilt, doch den eigenen Arbeitsplatz sieht man durch ein verstärktes Engagement nicht gefährdet.

Im Unterschied zu den Fällen mit schwach ausgeprägter Innovationsaffinität geht die Initiative zur Prozessoptimierung von Teams und betrieblichem Management gleichermaßen aus.

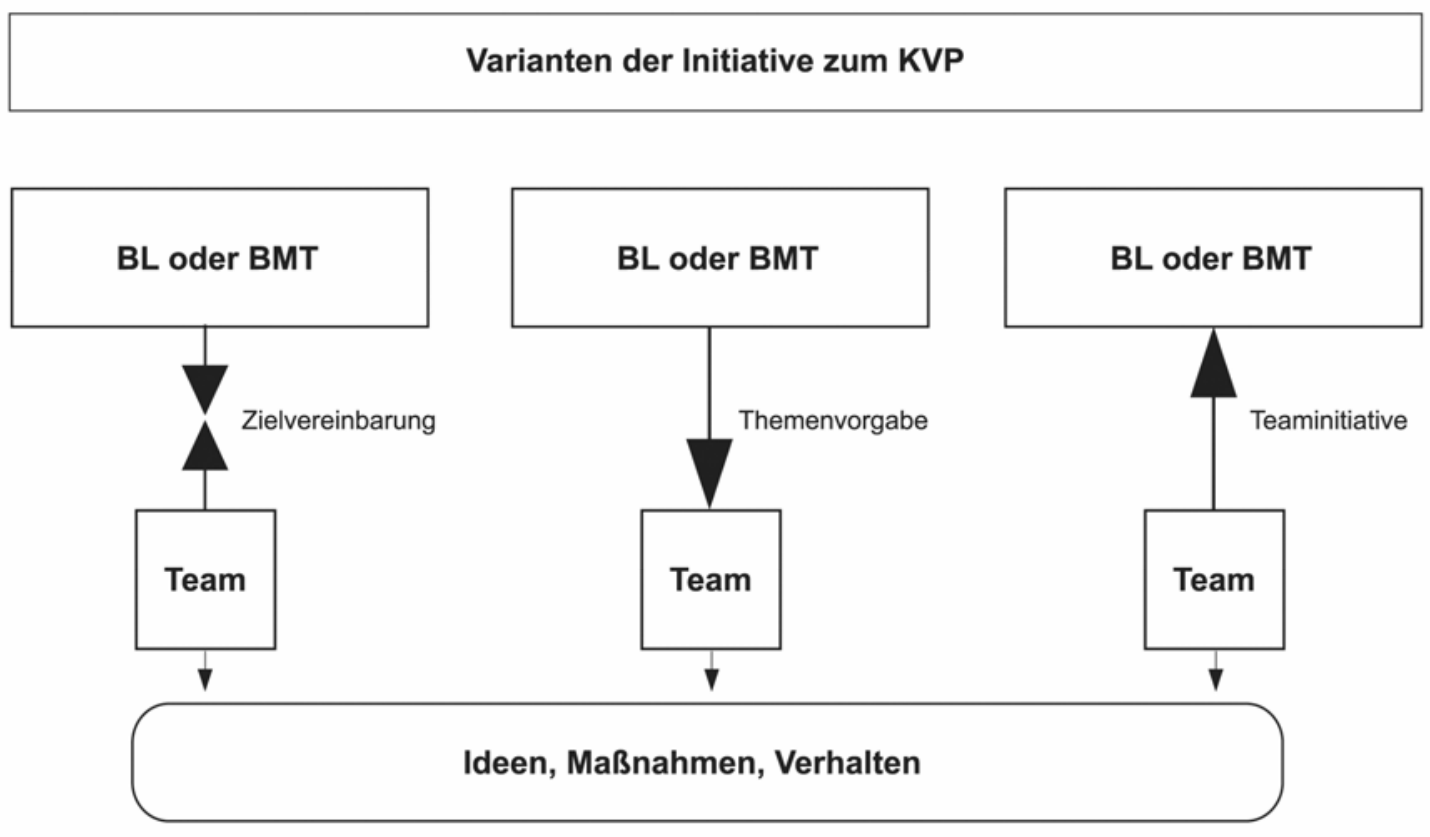

Abbildung 9: Prozessoptimierung im Betrieb

In den Betrieben werden zur Bearbeitung von Ideen ganz unterschiedliche Vorgehensweisen erprobt, Eine wichtige Rolle spielen in diesem Prozess die Gruppengespräche. Sie werden sowohl zur Entwicklung eigener Vorschläge aus den Teams heraus genutzt, als auch zur Bearbeitung von aus Sicht des Managements relevanten Problemfeldern. Das Management delegiert Aufgaben zur Prozess- und Anlagenoptimierung direkt als „Arbeitspakte“ in die Teams. Diese werden dann von den Schichtmannschaften bearbeitet. Oder das betriebliche Management vereinbart mit den Schichtmannschaften Themen, wie etwa den „Umgang mit Fehlern“ oder die „Urlaubsplanung“. Die Fragestellungen werden dann von den Teams bearbeitet. In jedem Fall werden die Ergebnisse den zuständigen 
Managementvertretern präsentiert und mit diesen sowie, falls diese ebenfalls thematisch betroffen sind, mit den zuständigen Fachgewerken diskutiert. Ein Vorgehen, das überaus positive Resonanz findet, und das den Stellenwert des Erfahrungswissens der Beschäftigten deutlich anerkennt.

Darüber hinaus werden Projektteams zur Bearbeitung komplexer, schichtübergreifender Fragen eingesetzt. Wie weit dies gehen kann, zeigt ein Beispiel im Fall PH. Hier hatte sich der Bereichsingenieur zum Ziel gesetzt, die Gruppen nicht nur Vorschläge ausarbeiten zu lassen, sondern auch an deren Realisierung mitzuwirken. Für diese Umsetzung wurde den Mannschaften ein kleines Budget bereitgestellt, in dessen Rahmen sie frei agieren konnten. Zusammen mit dem für die schlosserischen Zusatzqualifikationen zuständigen Ausbilder haben die Teams Planung, Entwicklung und Bau des Werkstücks selbständig übernommen und in enger Interaktion mit anderen Funktionen realisiert. An diesem Prozess wird bereits deutlich, dass sich auch die Kommunikations- und Kooperationsstrukturen verändert haben, und die „kurzen“ Benachrichtigungswege wie auch die direkte Zusammenarbeit zum betrieblichen Alltag werden. Außerdem präsentieren die Schichten die Ergebnisse ihrer Arbeit vor einem Expertenkreis. In diesem Rahmen selbst als „Experten der Praxis“ Gehör zu finden, wird von den Produktionsteams äußerst positiv bewertet. Die Aussage eines Beschäftigten ist beispielhaft für die Veränderungen, die die Umsetzung des Konzepts im Hinblick auf die Status-, Kooperations- und Kompetenzstruktur des Betriebs bewirkt.

„Die hören mehr auf uns jetzt. Ob das von oben vorbestimmt ist, dass weiß ich nicht. Aber
wenn ich an einen Ingenieur rantreten will, dann gehe ich halt zu ihm hin, klopf an und
dann hat der ein offenes Ohr und so. (...) Ich könnte, wenn ich da hingehe, bei jedem.
Auch beim Betriebsleiter, wenn ich da hin gehe und sage: ,Hör mal, kannst du mir da hel-
fen?’, dann kommt der gleich mit oder wir machen einen Termin und dann schauen wir
uns das an.“ (Beschäftigter AI/3)

Diese arbeitsorganisatorischen Veränderungen der neuen, durch das Projekt entstehenden betrieblichen Struktur sind aus Sicht der Betriebsleitung dazu geeignet, die erhöhte Innovationsdynamik mit der effektiveren Erschließung der Wissensbestände, der Enthierarchisierung von Kooperationsprozessen wie auch dem Abbau von Statusunterschieden zu parieren. Voraussetzung für eine solche Umsetzung des Projekts ist somit nicht nur, dass auf der inhaltlichen Ebene Spielräume für innovatorisches Handeln der Beschäftigten entstehen, sondern ein über die Akteure in den Schichtmannschaften hinausweisender Wandel betrieblicher Rollen und Strukturen. Auch auf diesen wirkt sich, wie wir zeigen werden, die Markt-, Produkt- und Innovationssituation des Betriebs je nach Konstellation hemmend oder förderlich aus. 


\subsection{Neustrukturierung von Hierarchie und Kompetenzen}

Dass die strategische Zielsetzung von Reorganisationsprojekten sowie deren Umsetzung erheblichen Einfluss auf die Neustrukturierung von betrieblichen Hierarchien und Kompetenzen haben, ist empirisch vielfach bestätigt. ${ }^{173}$ Gerade im Zusammenhang mit der Einführung selbstorganisierter Gruppenarbeit gilt eine Abkehr des betrieblichen Status-, Kompetenz- und Kooperationsmodells vom bürokratisch-hierarchischen Leitbild als unerlässlich, und insbesondere den Meistern kommt eine zentrale Rolle zu: Die Entwicklungsperspektiven der neuen Arbeitsorganisation hängen in hohem Maß von der Unterstützung durch die Industriemeister ab (Gerst 1998). Gleichzeitig sind diese durch Gruppenarbeit in erheblicher Weise von Veränderungen betroffen, die den Kern ihres Kompetenzprofils empfindlich berühren. So sehen sie sich mit neuen Verhaltensroutinen der Beschäftigten konfrontiert und müssen auf statussichernde Entscheidungs- und Machtbefugnisse verzichten. Die „Sozialfigur Meister“, die durch hohe Qualifikationen, relative Handlungsautonomie bei gleichzeitiger sozialer Orientierung hin zur Belegschaft geprägt ist (Reindl 1998), gerät damit unter einen teils erheblichen Druck. Wir werden im folgenden am Beispiel der Betriebs- und Schichtmeister zeigen, welche Konsequenzen sich in den betrieblichen Konstellationen für das Anforderungsprofil der Meister ergeben. Die Innovationsdynamik beeinflusst diese maßgeblich: Während in den Betrieben mit schwach ausgeprägter Innovationsaffinität die Funktionsmasse für ein neues Meisterprofil unterschüssig bleibt, konturiert sich in den Betrieben AI und PH ein neues Meisterprofil, dass die arbeitsgestalterische Umsetzung auf dem Shop floor maßgeblich positiv beeinflusst.

\subsubsection{Betriebsmeister: Vom Personalvorgesetzten zum Fachkoordinator}

Dass die Meister im Prozess der betrieblichen Reorganisation eine besondere Rolle inne haben, ist ein an sich überraschungsfreier Befund, stellt doch der Meister durch seine „doppelte Vermittlerrolle“ (Schumann, Gerst 1997) zwischen Fertigungsleitung und Arbeitskräften wie zwischen direkten und indirekten Bereichen eine wichtige Position im Sozialgefüge dar. Dies bezeichnet das zentrale Widerspruchsmoment der produktionsnahen Führungsposition und verweist auf ihren spezifischen Arbeitskrafttypus. Bei ihnen treffen die divergenten Interessenlagen von Management und Beschäftigten aufeinander, und so verfügen sie einerseits über eine erhebliche Machtstellung, stehen aber andererseits auch im spannungsreichen Feld zwischen „oben“ und „unten“. Das Management fordert von ihnen, für einen reibungslosen Produktionsablauf zu sorgen und an Effizienz ausgerichtete Entscheidungen zu treffen. Die Beschäftigten erwarten, dass sich die Meister für ihre Belange gegenüber dem Management stark machen. Die Meister erleben also unmittelbar Personalprobleme, Termindruck, Fehlentscheidungen, Mängel mit Material und Werkzeugen, mangelnde Koordination etc. Ebenso müssen sie unmittelbar mit den Reaktionen der Mitarbeiter darauf, wie Ärger, Absentismus und Fluktuation umgehen (Remdisch 1996:109). Insgesamt ist so eine Position bestimmt, die mit den täglichen Unwägbarkeiten ebenso zurecht kommen muss wie mit der Routine des Alltags, die als „man

\footnotetext{
${ }^{173}$ Für das (mittlere) Management vgl. Deutschmann et al. (1995); Faust et al. (2000); Baethge et al. (1995), für die Position des Meisters im Industriebetrieb Fischer (1993); Jauch (1997); Tullius (2003).
} 
in the middle“ weder mental noch positional eindeutig der Unternehmensleitung oder dem Shop floor angehören (Zündorf 1982). Verstärkt wird diese prekäre Situation durch ein „Verantwortungs-Kompetenz-Dilemma“ (Jauch 1997:52). Es resultiert aus der hohen Verantwortung für den Arbeitsprozess bei eingeschränkter Gestaltungsmacht und nur begrenzten Durchsetzungschancen, diesen Verantwortungsbereich zu beeinflussen.

Gerade im Rahmen von Reorganisationsmaßnahmen wird die Rolle des Industriemeisters in Frage gestellt. Dass sich die Verhältnisse im kapitalistischen Industriebetrieb nicht zu Gunsten der Meister entwickelten, stellt Littler (1982) sehr deutlich klar: "The traditional foreman's power started to be modified as soon as it had emerged from the decay of internal contract.” Dies gilt auch für die Chemische Industrie, denn mit der Akademisierung und dem Aufbau dezentraler Forschungs- und Entwicklungsabteilungen in Form von Betriebslaboratorien verloren die Meister sukzessive an Einfluss. Insofern ist die Meisterposition, die sich zwischen Management und Shop floor verorten muss, historisch betrachtet immer schon von Ambivalenzen und Statuskonflikten geprägt.

Im Rahmen der Umsetzung auf innovativer Arbeitspolitik basierender Konzepte wird die innerbetriebliche Verteilung von Status und Kompetenzen erneut in Frage gestellt. Schon Anfang der 1990er Jahre annonciert die Industriesoziologie eine Krise der „Spezialisten der Unspezialisiertheit“ (Bahrdt 1958:30). ${ }^{174}$ Pessimistisch mutmaßt Fischer (1993), dass sich mit der Verlagerung dispositiver Funktionen an die Beschäftigten die bis dahin den Meistern vorbehaltenen Räume „'freier und autonomer' Entscheidungs- und Weisungsbefugnis“ (Fischer 193:143) genommen würden. In diese Richtung weist auch die Beratungs- und Managementwissenschaftliche Literatur. Das Wort vom „Innovationshemmnis“ (Bargmann 1984) machte in Bezug auf die produktionsnahen Führungspositionen eine steile Karriere: Das mittlere und untere Management, insbesondere die Meister, werden als „Problemgruppen“ bezeichnet und als Hindernisse im Prozess der Reorganisation ausgemacht. Dass dieses Szenario nicht das einzig denkbare und schon gar nicht das Schlüssigste sein muss, belegte ebenfalls Fischer (1993). Er verweist darauf, dass die arbeitsgestalterischen Maßnahmen kein auf die Beschäftigten in der Produktion begrenztes Konzept bei ansonsten gleichbleibenden, tayloristischen Strukturen sind. Ganz im Gegenteil: Die Produktion insgesamt werde komplexer und anspruchsvoller, Koordinationserfordernisse stiegen. Die forcierte Prozessintegration erfordere deutlich mehr Flexibilität. Aus diesen veränderten Bedingungen könnten auch für die Meisterposition Vorteile erwachsen, denn es bestehen Chancen, dass Funktionen, die ehedem in Stabsabteilungen und sogenannte sekundären Bereichen angesiedelt waren, wieder zum Produktionspersonal und in die Werkstatt zurückkehren (Fischer 1993:161).

Die Frage nach Erosion oder Extension betrieblicher Steuerungs- und Kontrollfunktionen für die Meister wird Ende der 1990er Jahre in empirischen Studien zu den Auswirkungen arbeitsorganisatorischer Umgestaltungen tatsächlich recht eindeutig beantwortet: Von einer Erosion der Meister kann keine Rede sein, ein erheblicher Funktionswandel indes bleibt den Meistern nicht erspart (Behrens et al. 1996; Jauch 1997; Faust et al. 2000).

\footnotetext{
${ }^{174}$ Behrens et al. (1996) etwa betitelten ihren Beitrag zur Meisterdebatte „Der Industriemeister und der Strukturwandel in der Industrie - Neue Variationen zu einem alten Thema“ und stellen fest: „Wenn die Industriesoziologie den Industriemeister ins Visier nimmt, dann signalisiert das regelmäßig eine Meisterkrise.“ (S.25)
} 
In der Realität betrieblicher Reorganisation zeigt sich, dass die Entwicklung der Meisterposition in engem Zusammenhang mit konkreten betrieblichen Rationalisierungs- und Reorganisationsstrategien steht. Behrens et al. etwa argumentieren, dass vor allem das Primat der Ökonomie den Meister von der potentiell in der Position angelegten Funktion des „Motors der Veränderung“ zur „Verschleißteil-Option“ beeinflusse. Meisterkarrieren und Entwicklungspfade definieren sich darüber,,,wie unmittelbar harte ökonomische Zielvorgaben den Prozeß der arbeitsorganisatorischen Umstrukturierung bestimmen, bzw. (...) welche zeitlichen und materiellen Ressourcen für den Prozeß der Umstrukturierung zur Verfügung stehen“ (Behrens et al. 1996:33). Mehr Belastungen stehen durchaus auch für die Meister auf der Agenda, eine Erosion sehen die Verfasser nicht, für sie bleibt der Meister relevant als „Scharnier“ und „Vermittler“. Jauch et al. setzen die Entwicklung der Meisterrolle in einen engen Zusammenhang mit der Ausprägung des Reorganisationsprozesses und unterscheiden auf Grundlage unternehmerischer Dezentralisierungsstrategien unterschiedliche Meister-Konstellationen. Bei „Dezentralisierung nach dem Selbstorganisationsmodell“" werden Aufgaben und Kompetenzen an die Beschäftigten abgegeben. Für die Meister resultiert, so die Befunde, aus dieser Aufwertung der Produktionsarbeit empirisch eine Situation, die von den Betroffenen als eine Schwächung bzw. Bedrohung der Position wahrgenommen wird. Im Fall der „Dezentralisierung nach dem Intrapreneurmodell“ findet hingegen eine Verlagerung von Kompetenzen und Verantwortung auf die Ebene der produktionsnahen Führungskräfte statt, die eine deutliche Aufwertung dieser Position beinhaltet. Erkennen Jauch et al. darin eine positive Entwicklungstendenz, in der die Meisterfunktion „revitalisiert“ wird, weist eine weitere Konstellation auch ein erhebliches Gefährdungspotential aus: Die Neuschneidung von Kompetenzen und Verantwortungen markiert einen so deutlichen Bruch mit dem traditionellen Anforderungsprofil der Position, dass die Meister als Aufsteiger aus der Arbeiterschaft von akademisch ausgebildeten „Seiteneinsteigern“ (sprich Ingenieuren) verdrängt werden und die „Meister"wirtschaft künftig ohne Meister auskommt. In zwei Leitvorstellungen bringen Jauch et al. die Positivpole der Entwicklung und damit verbundene neue Kernkompetenzen der Meister auf den Punkt: „Die eine konzipiert den Meister als ,Moderator' teilautonomer Teams, als einen auf das Feld der Personalführung konzentrierten Spezialisten für ,human relations'. Die andere Leitvorstellung fasst den Meister dagegen als quasiunternehmerische Führungskraft, als einen relativ umfassend zuständigen und selbständig agierenden Produktionsmanager, bei dem sich technische Leitung und Personalverantwortung vereinigen“ (Jauch 1997:384). Zwischen diesen Polen identifizieren die Verfasser „eine Bandbreite von Formen mit fließenden Übergängen“ (ebd. 206), d.h. betriebliche Restrukturierung beinhaltet sowohl Elemente von Selbstorganisation als auch von Intrapreneurship $^{175}$ - mit in der Summe positivem Effekt für die produktionsnahen Führungskräfte. Wo Selbstorganisation und unternehmerischer Zuschnitt von Führungsrollen im Dezentralisierungsprozeß gleichermaßen eine Rolle spielen, stabilisiert sich die Position des Meisters auf neuem Niveau (ebd. 220).

Unsere Befunde belegen diese Neuakzentuierung der Meisterrolle in den Betrieben AI und PH mit stark ausgeprägter Innovationsdynamik: Die Betriebsmeister werden aus der

\footnotetext{
${ }^{175}$ In einer Fußnote merkt Jauch (1997)dazu an, dass sich „ganz verschiedene Formen der Verbindung von Selbstorganisation und der Aufwertung der Linie gegenüber indirekten Bereichen bzw. Stäben denken“ lassen und sieht daher auch „ganz unterschiedliche Folgen für die Meister“ (ebd.:206). Ob die Konstellationen und Konsequenzen für die Profile der Meisterfunktion tatsächlich so vielfältig und so unterschiedlich sind, bleibt freilich fraglich.
} 
Rolle des Personalvorgesetzten herausgenommen und gelten in den Betrieben als „Fachkoordinatoren“. Die direkte Steuerung des operativen Geschäfts fällt aus ihrem Anforderungsprofil heraus, und die Personalverantwortung wird auf die Schichtmeister verlagert. Zentral für diese Verlagerung ist der Gedanke, die Kernkompetenzen der Betriebsmeister in höherem Maß als zuvor für Innovationshandeln zu nutzen. Im Vordergrund stehen aus Perspektive der Betriebleitung nicht so sehr der theoretische Hintergrund, sondern die Prozesskenntnis und ihr fachliches Können und Wissen um die spezifischen betrieblichen Anlagen.

\begin{tabular}{|l|}
\hline \multicolumn{1}{|c|}{$\begin{array}{l}\text { Zentrale Merkmale des neuen Anforderungsprofils } \\
\text { der Betriebsmeister }\end{array}$} \\
\hline Produktionsplanung und -kontrolle \\
\hline Information der Betriebsleitung und der Schichtmeister \\
\hline $\begin{array}{l}\text { Kontrolle der Einhaltung gesetzlicher Vorschriften, firmeninterner Richtlinien und betriebsinterner Anweisungen sowie } \\
\text { anderer Regularien (cGMP-Vorschriften) }\end{array}$ \\
\hline Kostenkontrolle, Optimieren der Wirtschaftlichkeit \\
\hline Weiterentwicklung von Produkten und Verfahren sowie chemisch-technologische Änderungen \\
\hline Durchführung von Schulungen \\
\hline Koordination von produktionsunterstützenden Maßnahmen \\
\hline Pflege und Weiterentwicklung der betrieblichen Dokumentation. \\
\hline
\end{tabular}

Abbildung 10: Neues Anforderungsprofil des Betriebmeisters

Um dies zu ermöglichen, werden die Meister erstens in neuer Qualität in die betriebsinterne wie betriebsübergreifende Planung und Koordination eingebunden. Eine wichtige Rolle spielt dabei, dass die Betriebsleitung offensiv das Handeln der betrieblichen Akteure unter das vom Konzept vorgegebene Motto „Betriebliches Management im Team“ (BMT) stellt. Ingenieure (PLT und IT), Chemiker, Werkstatt- und Reparaturmeister, Logistik, Versand, Betriebsleiter und Produktionsassistent sowie die Betriebs- und Schichtmeister sind in einem Gremium zusammengefasst. Das Forum wird zur Koordination und Klärung betriebsrelevanter Projekte, zur Intensivierung betrieblicher Informationen und übergreifender Optimierung von Projektarbeiten genutzt. Sowohl die Betriebsleitung wie auch die Bereichsingenieure geben Aufgaben zur eigenständigen Erledigung in dieses fachfunktionsübergreifende Team. Das betriebliche Forum ist in diesen Fällen zum Koordinations- und Knotenpunkt von Innovationsaktivitäten geworden, in dem die Fäden von Arbeits- und Betriebsorganisation regelmäßig und institutionell verankert zusammenlaufen. Zugleich ist es durch seine fach ,hierarchie und funktionsübergreifende Ausrichtung in besonderem Maß Ausdruck einer auf Prozessorientierung basierenden betrieblichen Struktur.

Die Betriebsmeister sind in diesem Team, in dem betriebliche Innovationsvorhaben vorbereitet werden, als aktive Teilnehmer gefragt. Diese Zusammenarbeit geht insofern inhaltlich über die in den Betrieben regelmäßig stattfindenden Reparaturbesprechungen hinaus, als Themen diskutiert und bearbeitet werden, die nicht nur kurz-, sondern auch mittel- und langfristige Konsequenzen haben. Dies heißt dann beispielsweise, dass die 
Betriebsmeister wesentlich früher als bislang über Produktumstellungen informiert werden. Der Planungsvorlauf ist damit länger, und für die Betriebmeister ist dies ein wichtiger Punkt, um die Produktionsprozesse sicherer und optimierter zu gewährleisten. Außerdem werden Verbesserungen im Bereich Produkt, Produktion, und Organisation diskutiert, die über das Tagesgeschäft hinaus reichen. In Absprache mit den Fachgewerken und der Betriebsleitung definieren die Betriebsmeister Projekte technischer Art, die sie eigenständig lösen. Zusätzlich werden ihnen Aufgaben aus dem Kompetenzbereich der Ingenieure übertragen, etwa die Dokumentation und Pflege von Prüfplänen oder die Überarbeitung von Betriebsanweisungen.

Darüber hinaus steigt zweitens der Anteil betriebwirtschaftlicher Aufgaben. Im Fall AI etwa koordinieren nun anstelle der Betriebsleitung die Meister direkt die Zuliefer- und Abnehmerbeziehungen. Die Budgetverantwortung der Betriebsmeister wird zudem erheblich aufgestockt. Die Betriebsmeister müssen sich nicht mehr, wie bisher üblich, höhere Summen zur Materialbeschaffung vom Betriebsleiter quittieren lassen. Dieser Punkt ist, so belegt unsere Empirie, ein äußerst relevanter Faktor für die Umprofilierung der Betriebsmeisterposition. Sie ermöglicht das eigenständige Handeln, da die Projekte in weitaus höherem Maß ohne die direkte Kontrolle der Betriebsleitung zu bearbeiten sind. Aus Sicht der Meister wird damit ihre fachliche Autorität durch die Betriebsleitung anerkannt, und auch gegenüber betrieblichen Akteuren sichert dies ein souveräneres Auftreten.

\begin{tabular}{|l|}
\hline \multicolumn{1}{c|}{ Umprofilierung des Betriebsmeister } \\
\hline betriebliche Position \\
\hline $\begin{array}{l}\text { Bindeglied zwischen Prozessebene (Schichtmeister / Team) und Tagschicht (Chemiker / Ingenieure) sowie } \\
\text { Theorie und Praxis }\end{array}$ \\
\hline der Betriebsmeister behält: \\
\hline \begin{tabular}{l} 
Vertretungsfunktion der Betriebsleitung aufgrund seiner praktischen Betriebserfahrung und hoher \\
Prozesskompetenz \\
Assistenzfunktion bei der Betriebsleitung \\
Schichtübergreifende Koordinationsfunktion \\
\hline Umprofilierung in Richtung: \\
\hline Koordination der Schichtmeister statt Linienvorgesetzter und „Obermeister“ \\
Orientierung auf Fachaufgaben, Abläufe, Verfahren, Prozesssicherheit, Prozessoptimierung statt Tagesgeschäft \\
bzw. Feuerwehrfunktion \\
Projektarbeit, Mitarbeit bei betrieblichen Planungen
\end{tabular} \\
\hline
\end{tabular}

Abbildung 11: Neues Profil des Betriebsmeisters

Die Mehrheit der befragten Betriebsmeister bewerten diese Umprofilierung eindeutig positiv, entspricht diese Neufassung doch einem Spezialisierungsschub, der sie ganz eindeutig in Richtung Management-Funktion aufwertet. Anders formuliert: Verortete der Betriebsmeister in der traditionellen Arbeitsorganisation im Chemiebetrieb sich irgendwo zwischen koordinierenden, konzeptionellen aber eben auch operativen Aufgaben, spielt das operative Geschäft für seinen Arbeitsalltag jetzt nur noch eine untergeordnete Rolle. Diese Profilierung kommt dem Selbstverständnis der Betriebsmeister durchaus entgegen, haben sie doch bereits in der Vergangenheit ihre Expertise in der Vermittlung von theore- 
tischem Akademikerwissen mit praktischer Betriebserfahrung verortet. Die Abgabe von Personalverantwortung wird nicht als eine Einschränkung ihrer fachlichen Autorität wahrgenommen.

„Ich rieche Störungen und klemme mich dann so lange dahinter, bis ich was gefunden habe. Und jetzt habe ich einfach mehr Zeit, den Bau nach Fehlerquellen zu durchsuchen. (...) Ich kriege jetzt mehr mit, dadurch, dass ich Dinge abgeben konnte.“(Betriebsmeister $\mathrm{PH} / 1)$

Die Prozesshaftigkeit der Reorganisation bremst freilich zunächst die uneingeschränkte Konzentration auf Störungsbearbeitung, Fachkoordination sowie Projektarbeit. Die Zuständigkeiten und Entscheidungskompetenzen der Betriebsmeister gehen zwar bereits an die Schichtmeister und Teams. Sie aber müssen die neuen Anforderungen, die aus den veränderten Aufgabenprofilen resultieren, erst zur Handlungsroutine machen. Die Betriebsmeister sehen sich daher einerseits nach wie vor in der Verantwortung für betriebliche Abläufe.

„Wenn Sie mir sagen, dass ich ab morgen keine Verantwortung mehr habe für den Laden, dann lass ich hier alle machen, was sie wollen. Solange ich aber noch gerade stehen muss, muss ich wissen, was hier passiert.“ (Betriebsmeister AI/2)

Andererseits sind sie mit zunehmender Selbständigkeit und Mitspracheerwartungen „von unten“ konfrontiert und wollen dies auch unterstützen:

„Die Leute müssen erst mal merken: Wieweit kann ich denn nun eigentlich entscheiden?

(...) Wenn Sie wüssten, wegen welcher Sachen die mich manchmal noch nachts aus dem Bett holen...“ (Betriebsmeister AI/2)

Diese Effekte werden von den Meistern allerdings durchaus als Übergangsphänomene wahrgenommen und es gibt für sie keinen Grund, ihre positive Einstellung zu ändern. Ihr Fach- und Erfahrungswissen ist gefragt und sie sind gleichzeitig von den für sie eher lästigen Aufgaben der Personalführung befreit.

Eine Unsicherheit im Hinblick auf den Wegfall der Stelle des Betriebsmeisters gibt es bei den Meistern nicht. Im Gegenteil, sie sehen ihre Rolle durch die Reorganisation gestärkt und fühlen sich in ihrer Fachkompetenz bestätigt.

„Normalerweise kann man auf die Leute mit der besten Ausbildung nicht verzichten, im Gegenteil, deren Aufgaben erweitert man meistens noch.“ (Betriebsmeister HS/1)

Die Übertragung von Planungs- und Gestaltungskompetenz entschädigt die Betriebsmeister eindeutig für den Verlust an Einfluss, den sie durch die Verlagerung der Personalverantwortung an den Schichtmeister verlieren. In der Bilanz ist für die Betriebsmeister sogar ein Statusgewinn zu verzeichnen. Da ihre Fachautorität nun offiziell von der Betriebsleitung anerkannt wird, wächst auch ihre Autorität gegenüber den Schichtmeistern und den Beschäftigten. Dieser Sachverhalt wird von den betrieblichen Vorgesetzten auf Betriebs- und Abteilungsleitungsebene in den Interviews hervorgehoben. 
„Der Betriebsmeister bleibt die Seele im Betrieb. der hat die besten Anlagenkenntnisse, vor allem auch übergreifend, für die Verfahrensentwicklung ist das sehr wichtig. Der Betriebsmeister, das muss heute ein Coach sein, der für Schulungen, Training und eine bessere Betreuung der Mitarbeiter da ist. Deshalb muss der Betriebsmeister bleiben. Der hält alles zusammen und behält dabei den Überblick.“ (AL/Produktion)

Und ein Ingenieur berichtet von seinen Erfahrungen in den USA und stellt fest:

„Die Tagschichtmeister, die muss man lassen, deren Aufgaben kann man nicht umordnen, denn dann fehlt eine koordinierende Position im Betrieb. Ich kann ihnen sagen, da in (Ort), da gab es keine Tagschichtmeister mehr, da knirscht es wirklich!“ (BI/CI)

Neben der Tatsache, dass nun mehr Zeit für „Tüfteleien“ im Sinn von Prozessoptimierung bleibt, heben die Betriebsmeister die neue Qualität im Umgang miteinander, verstanden als kommunikative und kooperative Aspekte, als wesentliche Veränderung gegenüber der traditionellen Organisation hervor.

„Früher, da haben Betriebsmeister ja kaum was in Eigenregie regeln können, im Prinzip haben die nicht viel gemacht und waren das Sprachrohr der Betriebsleitung. Heute sind die Betriebsleitung und der Betriebsmeister eher wie Partner, der eine ist abhängig vom anderen. Das wird von denen (der Betriebsleitung A.d.V.) auch langsam kapiert.“ (Betriebsmeister $\mathrm{PH} / 2$ )

Dieser Wandel zeigt sich auch im Verhältnis zu den Schichtmeistern. Wo in der alten Struktur die Betriebsmeister eher direktiv führten, wird nun die neue „partnerschaftliche“ Beziehung betont. Exemplarisch dafür steht der von einem Betriebsmeister geschilderte Wandel innerhalb der Frühbesprechung. In ihr sitzen regelmäßig zu Beginn der Tagschicht Vertreter der Betriebsleitung, Betriebsmeister sowie der Schichtmeister der Frühschicht zusammen, um die tagesaktuellen Routinen abzustimmen, vor allem aber, um Störungsmeldungen und sonstige Unregelmäßigkeiten zu koordinieren. Vor der Umsetzung des Konzepts moderierte der Betriebsmeister die Sitzungen und erläuterte mittels des technischen Schichtbuchs, was aus seiner Sicht an Maßnahmen einzuleiten sei. Vom Betriebsleiter wird die Sitzung retrospektiv als „Morgenandacht“ bezeichnet: Die Schichtmeister saßen zumeist „still und andächtig“ dabei, erst auf Nachfrage nahmen sie Stellung zu Details. Heute gebe es neue „Spielregeln“, die sich vor allem in einer Aufwertung der Schichtmeister manifestierten. Statt passiv als „Zuhörer“ sind die Schichtmeister nun in einer aktiven Rolle gefordert. Sie leiten die Sitzung, und es wird von ihnen erwartet, dass sie die relevanten Informationen aus dem technischen Schichtbuch vorab sammeln und eine erste Bewertung vornehmen bzw. Vorschläge zur Bearbeitung vorlegen. Zusammen mit den Vertretern der Betriebsleitung werden dann die einzelnen Punkte diskutiert und gemeinsam Lösungen erarbeitet.

„Das ist schon erstaunlich, was da passiert ist. Obwohl wir da ja mit genau der gleichen Gruppe wie vorher zusammensitzen, ist der Dialog jetzt ein ganz anderer, das ist schon eine neue Gesprächskultur. Irgendwie haben wir wohl alle gelernt, dass die Besprechung fachlicher Dinge nur dann Sinn macht, wenn derjenige sie vorbringt, der am meisten damit zu tun hat.“ (Betriebsmeister PH/2) 
Insgesamt sehen die Betriebsmeister in dieser Entwicklung eine Verschiebung ihrer Kernkompetenz von kontrollierenden zu steuernden und fachlichen Aspekten, die für sie eine konsequente Schlussfolgerung der durch das Konzept inspirierten Verlagerungen von Kompetenzen und Entscheidungen im Betrieb bedeutet. Zumindest in der Kontrollfunktion sei denn auch der Betriebsmeister „überflüssig“, denn

„Wenn jeder seinen Job macht, dann braucht man die Betriebsmeister als Kontrollinstanz nicht mehr.“ (Betriebsmeister AI/1)

Hintergrund dafür ist ein in Anbetracht der hohen Innovationsdynamik verändertes Anforderungsprofil des betrieblichen Managements. Der Abbau mehrerer Hierarchieebenen auf Geschäftsfeld- und Abteilungsebene hat zur Folge, dass die Betriebleitung zunehmend selbst in permanentem Austausch mit anderen Einheiten stehen muss, um über Ressourceneinteilung und -nutzung zu verhandeln. Zusätzlich sind gerade die Betriebsleiter in $\mathrm{PH}$ und AI infolge der hohen Innovationsdynamik stark in betriebsübergreifende Forschungs- und Entwicklungstätigkeiten eingebunden. Sowohl diese Einbindung in die Projektorganisation als auch die qualitativ wie quantitativ zunehmende Dichte von Informations- und Kommunikationsprozessen erweitern die Anforderungen an die betrieblichen Führungskräfte in fachlicher wie in sozial-kommunikativer Perspektive. Dies wirkt sich auf die betriebliche Praxis insofern aus, als in AI und PH die Anteile, die das betriebliche Management für Gremienarbeit und Projektsitzungen aufwenden muss, erheblich steigen und die Spielräume für direkte Personalführung und Prozesskontrolle im Betrieb enger werden. Im Fall PH und AI etwa ist die Einbindung der Betriebsleitung in externe Projekte und Gremien so stark, dass die Ressourcen zur Bewältigung der anstehenden Probleme nicht mehr ausreichen: In Gesprächen mit den Betriebsleitern wurden Abwesenheitszeiten zwischen 50 und 70 Prozent für diese Tätigkeiten veranschlagt. Zugleich ist der Bedarf an Planung, Steuerung und Optimierung vor Ort hoch, und in unseren Gesprächen mit den Betriebleitern betonten diese, dass man sich unter diesen Voraussetzungen „auf die Leute vor Ort hundertprozentig verlassen muss“ (Betriebsleiter AI). In Anbetracht der Potentiale für Produkt- und Verfahrensverbesserungen kommt es in den Fällen AI und PH zu einem aus der Neuaufteilung der individuellen manageriellen Ressourcen resultierenden „Kaskadeneffekt“, denn unter den hohen Anforderungen, die sich aus der Innovationsdynamik ergeben, „kann die Führungskraft, die selbst mehr Verantwortung und Kompetenz erhält, nur ,überleben', wenn sie ihrerseits nach unten Kompetenzen abgibt und Verantwortung teilt“ (Faust et al. 1997:9). Um unter diesen Prämissen eine Produktion aufrecht zu erhalten, die hohe Ansprüche an Flexibilität und Innovationshandeln stellt, gibt die Betriebsleitung in sehr weitreichendem Maß Aufgaben und Entscheidungskompetenzen ab, und auch für die Betriebsmeister erhöht sich die Verantwortung und werden neue Kompetenzen gefordert. Die Betriebsleitung verfolgt dabei das Ziel, sich auf die betriebsstrategischen Aufgaben zu konzentrieren, und delegiert dispositive und operative Aufgaben sowie die dafür notwendigen Entscheidungsbefugnisse „dorthin, wo Können und Erfahrung vorhanden sind“ (betriebsinterne Folie).

Zwar ist dies eine Vorgabe die auch in den Betrieben KS und PF zur Leitlinie des betrieblichen Managements wird. Allerdings ist sie dort, so unsere Ergebnisse, insgesamt eher als Lippenbekenntnis zu verstehen. Am deutlichsten wird dies in den auch in diesen Fällen praktizierten BMT-Sitzungen. In der Regel diskutieren während der Sitzungen die Gruppen miteinander, die ohnehin miteinander kooperieren. Die Treffen unterscheiden 
sich deshalb nicht von den Reparatur- oder Frühbesprechungen. Die in den Betrieben AI und $\mathrm{PH}$ nach einigen Anlaufschwierigkeiten recht gut funktionierende gemeinsame Projektbearbeitung und die Entwicklung von Problemlösestrategien hingegen findet mangels thematischer Vorgaben nicht statt.

„Oft ist es so, dass nur drei von zwanzig Leuten reden, der Rest sitzt dann da, dreht Däumchen und schläft fast ein. Wirklich interessante Sachen für alle werden da eigentlich nicht besprochen, das ist meist nur Pipifax.“ (Betriebsmeister KS/3)

Für die Betriebsmeister hat dies zur Folge, dass ein Neuzuschnitt im Sinn einer Aufwertung durch die Übernahme von Aufgaben aus dem verantwortungs- und Kompetenzbereich des betrieblichen Management ausbleibt. Zugleich sehen sich die Betriebsmeister in diesen Betrieben damit konfrontiert, dass das Konzept die betriebliche Hierarchie um eine Stufe reduziert und die Schichtmeister gleichrangig mit den Betriebsmeistern in die betrieblichen Strukturen integriert werden sollen. In dem Maß, in dem Verantwortung und Kompetenzen der den Betriebsmeistern unterstellten Beschäftigten übertragen werden, wird die Funktionsmasse von Entscheidungs- und Anweisungsbefugnissen kleiner. Da in diesen Fällen die Innovationsdynamik nicht den betrieblichen Aufwand an Planung, Steuerung und Optimierung insgesamt erhöht, wird zwar von einer „neuen Rolle“ der Betriebsmeister gesprochen, diese bleibt jedoch ohne inhaltliche Konkretisierung. So kann der Funktionsverlust durch die Verlagerung von Aufgaben in die Schichten nicht kompensiert werden.

Von den Betroffenen wird deshalb das Reorganisationsprojekt in erster Linie als ein Rationalisierungsvorhaben verstanden, dass womöglich den Verlust des eigenen Arbeitsplatzes implizieren kann. Da es den Meistern nicht möglich ist, eigenständig Funktionen aus übergeordneten hierarchischen Ebenen an sich zu ziehen, überrascht es wenig, dass sie in dieser Situation alles daran setzen, möglichst wenige ihrer Kernaufgaben abzugeben. In den Gesprächen setzen die Befragten alles daran, Argumente dafür zu finden, dass sie ihre traditionellen Weisungsbefugnisse und Einflussmöglichkeiten in jedem Fall behalten müssen. Begründet wird dies in erster Linie damit, dass die Teams nicht die nötige Kompetenz sowie das Erfahrungswissen haben, und es heißt dann oft „Das sehen die gar nicht“ oder „Davon verstehen die doch nichts“. Die Meister heben speziell das in der Chemischen Produktion liegende Risikopotential hervor und betonen, dass die neuen Hierarchien für „Verwirrung“ sorgen und „ganz objektiv“ eine Unsicherheit für den Produktionsprozess darstellen. Vor allem das Prinzip der „kurzen Wege“ wird in seinen Auswirkungen negativ bewertet, da es den Meistern nun unmöglich sei, die Übersicht über den Status quo der Anlagen zu behalten.

„Die rennen jetzt sofort zum Doktor (Betriebsleiter, A.d.V.), das muss man sich mal vorstellen. Und ich weiß dann nicht bescheid, so geht das doch nicht. Das ist schlecht fürs Produkt.“ (Betriebsmeister KS/2)

Vor allem die Personalhoheit wird als wichtiges Herrschaftsinstrument angesehen, und es ist den Meistern ein Dorn im Auge, dass dies nun von den Schichtmannschaften selbst erledigt werden soll. In den Gesprächen wird deutlich, dass die Meister vor allem die Personalplanung sowohl im Hinblick auf Urlaub und Arbeitseinteilung als auch auf die Qualifizierungspläne als zentrales Steuerungsinstrument betrachten und dies wieder in ihre Hände bekommen wollen - nötigenfalls auch mit erheblichem Druck gegenüber den Be- 
schäftigten. Für die Schichtmannschaften werden so die Spielräume zur Selbstorganisation erheblich beschnitten, und es überwiegen bei der Umsetzung des Gestaltungsansatzes die erhöhten Belastungen durch die Arbeitsverdichtung. Aus Sicht der Beschäftigten ist darüber hinaus das Verhalten der Vorgesetzten ein wichtiger Grund für das schlechte Betriebsklima.

„Da kommt nichts bei rum (bei dem Projekt). Das Klima ist schlecht, die Leute sind angespannt, wenn hier einer was sagt, dann explodieren gleich fünf, sechs Leute.“ (Beschäftigter $\mathrm{KS} / 1)$

Die Meister spielen ihre Machtposition aus und nehmen den Beschäftigten die wenigen Möglichkeiten zur Selbstorganisation durch eine konsequente Präsenz in den Teamgesprächen. Aufgaben und Verantwortungen, die den Betriebsmeistern formal nicht mehr zustehen, werden von ihnen unter der Hand wieder übernommen, und insbesondere die Gruppengespräche sind für die Meister ein effektives Forum zum Erhalt des innerbetrieblichen Status Quo.

„Teamgespräche, ja, klar, aber nie ohne den Chef und den zweiten Mann. Das ist doch klar, was das heißt: Wir hier unten, wir sind die kleinen Arschlöcher.“ (Beschäftigter $\mathrm{PF} / 4)$

Statt kooperativem Führungsstil und Coaching fallen die Betriebsmeister dabei teilweise in tradierte Strukturen zurück und verschärfen den autoritären Führungsstil noch gegenüber der Zeit vor der Reorganisation.

„Die Meister hier, die sind völlig bekloppt, die regieren wieder mit der Peitsche, so wie vor fünfzig Jahren.“ (Beschäftigter KS/4)

In den Schichten provoziert dieses Verhalten einen erheblichen Unmut, da sich die Betriebsmeister nicht an die „neuen“ Regeln halten und dies als klarer Übergriff in die neuen Kompetenzbereiche gewertet wird:

„Die Tagschichtmeister, die haben Teamarbeit nicht begriffen, und zwar alle drei nicht. Die sagen immer noch , der macht das und das' obwohl sie das bei Teamarbeit nicht dürfen. Normalerweise muss er mir die Aufgaben übergeben und ich gebe das dann weiter an die Gruppe. Die entscheidet dann.“ (Schichtmeister PF/1)

Wesentlich befördert wird das widerständige Verhalten der Betriebsmeister noch durch die Indifferenz der Betriebsleitung dem Projekt gegenüber.

„Die (Betriebsmeister) wurden dazu verdonnert (Teamarbeit zu machen), so kann man natürlich nicht anfangen. Der Betriebsleiter hat dann einfach gesagt: Macht ihr mal. Und die haben dann natürlich gesagt: Hier ändert sich nichts.“ (Prozessbegleiter 2/8)

Zusammenfassend ist festzustellen, dass die Meister den Funktionsverlust auf der Ebene der Personalverantwortung mit einem erheblichen Statusverlust gleichsetzen. Ihnen wird ein Großteil an Anordnungsbefugnissen genommen, gleichzeitig erfahren sie aber keine verstärkte Anerkennung ihrer Sachautorität in Form neuer Verantwortlichkeiten. 
Dieses Phänomen bleibt indes nicht auf die Betriebe PF und AI beschränkt. Auch in den Betrieben mit starker Innovationsaffinität sind nicht alle Betriebsmeister gleichermaßen in die Umprofilierung mit einbezogen. Im Betrieb PH etwa hat der Betriebsleiter den Betriebsmeistern jeweils ein spezielles Aufgabenprofil zugeordnet. Hintergrund dieser Aufgabenverteilung sind einmal die chemiespezifischen Sicherheitsauflagen, die eine personelle Verantwortlichkeit für bestimmte Aufgaben vorsehen. Dass sich das betriebliche Management bei der Aufgabenverteilung an den Kompetenzen der jeweiligen Betriebsmeister orientiert, kommt in diesem Statement sehr deutlich zum Ausdruck.

„Der (X), der ist jetzt für die Prozessoptimierung und die Schwachstellenanalyse zuständig, der ist ein sehr guter Mann dafür, der kennt den Betrieb wie seine Westentasche. Der (Y), der ist erst seit kurzem hier im Betrieb, der macht die Validierung, Qualitätssicherung und GMP. Und der (Z), der ist für die Versorgung zuständig, also alles, was so im sozialen Bereich anfällt...(zögert) der kommuniziert auch ein bisschen nach außen. Das ist sicherlich der am wenigsten attraktive Bereich, da wird die Meisterqualifikation schon in Frage gestellt. Die Stelle würde ich im Moment, wenn ich sie neu besetzen müsste, nicht mit einem Meister besetzen.“ (BL PH/1)

An diesem Beispiel wird deutlich, dass es auch in der Chemischen Industrie zukünftig nicht um eine Abschaffung der Meisterposition gehen wird. Der rationalisierungswirksame Aspekt der Reorganisation liegt eher in einer Aufwertung der Funktion bei gleichzeitiger Reduktion der Stellen.

\subsubsection{Vom Schichtführer zum Schichtmeister und Coach}

Während in den Betrieben mit schwach ausgeprägter Innovationsaffinität auf der Ebene der Schichtmeister eine Neustrukturierung von betrieblicher Hierarchie und Kompetenzen ausbleibt, verändern sich in den Betrieben AI und PH parallel zum Profil der Betriebsmeister auch die Anforderungen an die Schichtmeister.

\begin{tabular}{|l|}
\hline \multicolumn{1}{|c|}{$\begin{array}{l}\text { Zentrale Merkmale des neuen Anforderungsprofils } \\
\text { der Schichtmeister }\end{array}$} \\
\hline Abwickeln der Produktion \\
\hline Kontrolle von Ausbeuten und Einhaltung von Sollvorgaben \\
\hline Bewerten von Abweichungen \\
\hline Einleiten geeigneter Maßnahmen bei Störungen \\
\hline Kontrolle und Bewertung der Qualifikation der Gruppen \\
\hline Initiieren von Weiterbildungsmaßnahmen, Durchführen von Schulungen \\
\hline Kontrolle der Produktionsdokumentation \\
\hline Erarbeiten und Aktualisieren von Arbeitskontrollbögen und Betriebsanweisungen \\
\hline Fachliche Berichterstattung an die Vorgesetzten \\
\hline
\end{tabular}

Abbildung 12: Neues Anforderungsprofil des Schichtmeisters 
Ebenso wie die Betriebsmeister werden die Schichtmeister aktiv in Gremien- und Projektarbeit mit einbezogen. Sie nehmen an Frühbesprechungen oder Reparatursitzungen teil, und ihre Expertise wird auch in der schichtübergreifenden betrieblichen Planungen stärker berücksichtigt. Wie wichtig diese stärkere Einbindung in die Tagschicht den Schichtmeistern ist, belegt der Fall PH. Da wegen der rotierenden Schichtpläne die Teilnahme der Schichtmeister durch Diskontinuität geprägt ist, haben die Meister in Eigeninitiative Schichtmeister-Treffen organisiert. Sie tauschen sich auf diesem Weg über fachliche wie soziale Belange aus. Der schichtinterne Informationsfluss wird so koordiniert wie auch die Schnittstelle zwischen Tag- und Wechselschicht optimiert.

Dieser Umprofilierung ist auch für die soziale Struktur der Schichtmannschaften folgenreich. Statt „erster Mann“ der Schicht zu sein, überlassen die Schichtmeister mit verändertem Profil das Produktionsgeschehen sowie Koordinationsaufgaben weitgehend den Teams. In Stresssituationen arbeiten sie zwar in der Schicht mit, kümmern sich aber ansonsten verstärkt um Prozessoptimierung und schichtübergreifende Außenkontakte. Für die Mannschaften sind sie zunehmend Coach, der bei Konflikten in der Schicht wie zwischen Schicht und Management vermittelt. Der Meister trägt dafür Sorge, dass die Gruppen die notwendigen Informationen über relevante Kennzahlen, betriebliche Abläufe und Prozesse erhalten. Ihre Funktion reicht aber weit über diese „Bringleistung“ hinaus, und ihr Einfluss, ihre Expertise und ihre Erfahrung sind vor allem dann gefragt, wenn Autorität, Durchsetzungschancen und Kompetenzen der Beschäftigten an ihre Grenzen stoßen. ${ }^{176}$

\begin{tabular}{|l|l|}
\hline \multicolumn{2}{|c|}{ Veränderung der Meisterrolle im Hinblick auf die Personalführung } \\
\hline Abnehmende Bedeutung & Zunehmende Bedeutung \\
\hline $\begin{array}{l}\text { Führen und direktes Anweisen von „Einzelkämpfern“ mit } \\
\text { unterschiedlichen Kompetenzen }\end{array}$ & $\begin{array}{l}\text { Führen von Gruppen mit zunehmend einheitlichem } \\
\text { Kompetenzprofil und hoher Selbständgkeit }\end{array}$ \\
\hline Einteilen der Arbeit: Wer macht was bis wann und wie. & $\begin{array}{l}\text { Rahmen für die Gruppen festlegen, Spielregeln } \\
\text { vereinbaren, Verantwortung delegieren }\end{array}$ \\
\hline Schichtmannschaften kontrollieren & Schichtmannschaften entwickeln \\
\hline Blick auf Individuum und Einzelleistung & Teamfähigkeit und Teamleistungen fördern \\
\hline Rolle als Anweiser und Überwacher & Rolle als Förderer, Unterstützer und Entwickler \\
\hline
\end{tabular}

Tabelle 7: Meister und Personalführung

Von den Schichten, in denen die Schichtmeister in diesem Sinn auftreten, werden sie als wichtiger „Experte der Praxis“ zu Rate gezogen. Ihre Scharnierfunktion zwischen Tagund Wechselschicht bleibt erhalten, erfährt aber eine deutliche qualitative Veränderung. Die Schichtmeister behalten im Idealfall den Überblick über Prozess und Mannschaft, die

\footnotetext{
${ }^{176}$ Beispiele für solche Koordinationsleistungen sind die Beschaffung von Werkzeugen, der Ausgleich von Personalengpässen, aber auch der Zugriff auf finanzielle Ressourcen, die Beschaffung von Schulungsräumen und die Organisation von Schulungsmaßnahmen. (Antoni 1994; Gerst 1998)
} 
operativen Zuständigkeiten aber werden den Teams überlassen. ${ }^{177}$ Als „Coach“ unterstützen die Meister die gruppengetragene Selbstorganisation, indem sie beispielsweise die für den KVP notwendigen Ressourcen bereitstellen. Es kann aber auch bedeuten, dass die Schichtmeister auf Wunsch der Gruppen an den Gruppensitzungen teilnehmen, um beispielsweise als Experten Verbesserungsvorschläge der Teams zu diskutieren, bevor diese als offizielle Gruppenvorschläge eingereicht werden. Gleichzeitig verstehen sich die Schichtmeister nicht mehr als „bester Mann“ an jeder Anlage - und müssen dies auch nicht mehr, da sie auf die Kompetenz ihrer Schichtmannschaften vertrauen können. Daraus folgt für die Meister, dass sie neue „Ungewissheitszone“ in Kauf nehmen müssen, denn im Rahmen der von den Gruppen praktizierten Selbstorganisation wird nicht mehr jede Unregelmäßigkeit im Prozess an den Schichtmeister weitergeleitet, sondern innerhalb der Gruppe diskutiert. Der soziale Bezugsrahmen, den die Schicht gerade in der Chemischen Industrie auch für die produktionsnahen Führungskräfte bildet, wird damit in Teilen geöffnet. Der Meister ist nicht länger der „väterliche Freund“, der Alle(s) kontrolliert. Zwar behalten die Schichtmeister ihre Weisungsbefugnis gegenüber den Mannschaften, im Gegensatz zu früher können die Teams aber selbst entscheiden, wann und wie sie die ihnen übertragenen Aufgaben bearbeitet.

„Früher, da konnte ich sagen, Du, mach mal, das hieß dann „sofort“. Heute sage ich der Gruppe: Bis acht Uhr müssen die Fässer da und da sein. Den Rest organisieren die..."(Schichtmeister AI/2)

Dass diese Umprofilierung nicht so reibungslos funktioniert, ist vor allem den chemiespezifisch eng gefassten Regelungen zur Delegation und der Übertragung von Verantwortung geschuldet. Eine Verantwortung, die von einer gesamten Gruppe ausgeübt wird, kann es aus GMP-bedingten Gründen nicht geben. Für jeden dokumentierten Schritt muss es im Nachhinein die Möglichkeit geben, ihn einer Einzelperson zuzuweisen. So stehen die Schichtmeister nicht selten vor dem Dilemma, für Vorgänge verantwortlich zu zeichnen, die zwar nicht in Gänze aber doch in weiten Teilen jenseits ihrer direkten Kontrolle, d.h. innerhalb der Schichten ablaufen.

„Hier muss jeder Schritt erst vom Mitarbeiter und dann von mir unterschrieben werden. Wie soll ich das machen? Eigentlich müsste ich bei jeder Unterschrift mit dabei sein. Das wäre aber dann die absolute Kontrolle, so wie früher...“ (Schichtmeister PH /1)

Die Führungs-, Aufsichts- und Kontrollpflicht der Schichtmeister, die bei aller gruppengetragenen Selbstorganisation bestehen bleibt, verlangt den Meistern eine erhebliche soziale Kompetenz ab, die eine Gratwanderung zwischen kooperativer Unterstützung und kleinschrittiger Kontrolle bedeutet. Nicht selten ist daher gerade in Stresssituationen, bei größeren Störungen etwa, einen Rückfall in den alten Anweisungsstil zu beobachten. Ähnlich wie auch für die Position des Betriebsmeisters steht im Bezug auf Kommunikations- und Informationsprozesse eine einschneidende Modifikation des Führungsstils sowie eine Verschärfung des Verantwortungs-/Kompetenz-Dilemmas an. Denn war die konkrete, personenbezogene Anweisung in der traditionellen Arbeitsorganisation Mittel zur Vermittlung zwischen betrieblichen Vorgaben und Ausführung durch die Beschäftigten, gilt nun der Modus der indirekten Steuerung, d.h. die Meister sind angehalten, die Ar-

\footnotetext{
${ }^{177}$ Dies schließt nicht aus, dass die Schichtmeister bei personellen Engpässen oder in produktionsbedingten Stresssituationen nach wie vor als Springer ins operative Geschäft eingreifen.
} 
beitsaufgaben so zu definieren, dass die Gruppen im Rahmen ihrer selbständigen Aufgabenerfüllung die Details innerhalb der Gruppe regeln können. Die Vermittlerrolle der Meister hat Bestand, findet allerdings auf einem deutlich anderen Niveau statt, da sie sich auf einen partizipativen Umgang stützt.

\begin{tabular}{|c|}
\hline neue Rolle des Schichtmeisters \\
\hline betriebliche Position \\
\hline $\begin{array}{l}\text { betrieblicher Vorgesetzter mit Führungsfunktion } \\
\text { direkte Unterstellung unter Betriebsleitung } \\
\text { förmliche Einbindung in das Betriebsmanagement }\end{array}$ \\
\hline Aufgabenstellungen bei Abgabe operativer Tätigkeiten an die Teams \\
\hline $\begin{array}{l}\text { Verantwortung für Prozess-Sicherheit, -Qualität und Optimierung sowie Personal } \\
\text { übergreifende Koordinations- und Informationsfunktionen } \\
\text { Verabredung von Zielvereinbarungen mit dem Team und Zielverfolgung } \\
\text { Unterstützung der Team-Selbstorganisation und -Entwicklung }\end{array}$ \\
\hline betriebsstrategische Zielperspektive \\
\hline $\begin{array}{l}\text { verantwortlicher „Meister“, Repräsentant der Scharnierfunktion zur Mannschaft (statt Vorarbeiter / Schichtführer) } \\
\text { Bindeglied zwischen Schicht- / Prozessebene und Betriebsmanagement }\end{array}$ \\
\hline
\end{tabular}

Abbildung 13: Neues Profil des Schichtmeisters

An der Frage, ob der Schichtmeister den Teams mehr „zutraut“ und sich selbst nicht mehr als den besten Anlagenfahrer versteht, wird dies besonders deutlich. Zum einen sagen diese Meister in den Gesprächen dezidiert, dass sie nicht die Besten sein könnten, die Komplexität der Anlagen ließe dies nicht mehr zu. Zum anderen haben sie diesen Anspruch auch nicht, sondern betonen, dass sie sich da auf die Beschäftigten verlassen würden. Zwar bleibe der Prozess unter ihrer Kontrolle, in jedem Fall zähle aber das Knowhow und die Kompetenz desjenigen, der gerade die Anlage fahre. Dass sich dieses auf das Standing der Beschäftigten auswirkt, wird sowohl von den Schichtmeistern bemerkt als auch von den Beschäftigten als qualitativ anderer Umgang mit ihrer fachlichen Kompetenz bewertet.

„Die Leute sind schon selbstbewusster geworden, sie fühlen sich nicht mehr so gedeckelt.“(Schichtmeister AI/4)

Ein Großteil der Beschäftigten schließt sich der Meinung an, dass man nun gegenüber dem Schichtmeister eine stärkere Stellung bekommen habe, die vor allem fachlich begründet ist.

„Zum Schichtmeister gehe ich jetzt nur noch bei bestimmten Störungen, die ich nicht selbst in den Griff krieg oder wenn ich im Labor eine Freigabe nicht machen kann, weil ich da meine Kompetenz überschreite. Aber unser Meister, also, der sagt dann auch immer: ,Kannst du das nicht selber machen?’ Der versucht, dass jeder mehr selbständig arbeitet.“ (Beschäftigter AI/2) 
Gerade die Chemikanten scheinen dabei in den Betrieben eine Kompetenzbasis zu bilden, die den Meister im Hinblick auf das operative Geschäft langfristig auch obsolet machen könnte, wenn es die gesetzlichen Vorgaben zuließen.

„Wenn sie hier gut ausgebildete Leute, Facharbeiter, haben, dann brauchen sie keinen Schichtmeister mehr. Uns wird hier zwar eingehämmert, dass wir am Wochenende doch die Vorgesetzten sind. Aber das ist ja eigentlich auch nur wegen der Gesetze.“ (Schichtmeister $\mathrm{AI} / 5$ )

Dies unterstreicht noch einmal den Wandel, der sich innerhalb der Belegschaften in der letzten Dekade vollzogen hat. Das Gros der Chemikanten verfügt heute über fachliche Kompetenzen, die von den Unternehmen bedingt durch die Vernachlässigung arbeitspolitischer Konzeptualisierungen verkannt bzw. schlicht nicht abgerufen wurde.

Die Schichtmeister werden durch die gruppengetragene Selbstorganisation von einem Teil ihrer klassischen Aufgaben entlastet, die Kernbereiche ihrer Tätigkeiten und Anforderungsprofile ausmachten. Dies setzt voraus, dass die Schichtmeister diese Aufgaben auch abgeben. Unsere Befunde zeigen: Eine Minderheit der Meister ist nicht bereit, die alte Kontrollfunktion und damit verbundene Einflussmöglichkeiten aufzugeben. Für die Schichtmannschaften ist dies folgenreich, denn selbst wenn sie eine hohe Motivation zeigen, Aufgaben des Meisters zu übernehmen, kann dieser dies verhindern. Eine Einschätzung, die auch von den betroffenen Meistern geteilt wird:

(Frage: Kann man als Meister die Teamentwicklung positiv beeinflussen, voranbringen?) „Also, ich kann es positiv beeinflussen, ich kann es auch blockieren. Hundertprozentig! Wenn ich will, kann ich das alles auch rechtfertigen, schließlich bleibt ja die Verantwortung an mir kleben.“ (Schichtmeister PH/1)

Somit verfügen die Meister nach wie vor über Ressourcen, mit denen sie unter der Hand die bestehende Ordnung aufrecht erhalten können. Dies engt die Freiräume für eine Teamselbstorganisation ein, da Entscheidungen der Teams nach wie vor der Willkür des Schichtmeisters unterliegen. Stellvertretend für diese Teams meint ein Beschäftigter:

„Solange sich der Schichtmeister sträubt, solange gibt es hier keine Teamarbeit.“ (Beschäftigter $\mathrm{PH} / 3$ )

In der betrieblichen Praxis konnten wir auch in den elaborierten Fällen AI und PH Teams finden, in denen formal alle Voraussetzungen der gruppengetragenen Selbstorganisation erfüllt sind, der reale Zuwachs auf der Entscheidungsebene für die Beschäftigten aber gering ist. Ihre Entscheidungen bzw. Optimierungsvorschläge sind vom good will der Schichtmeister abhängig. Je nach Tageslaune nimmt dieser die Schichtgruppen ernst oder bremst ihre Vorschläge gegenüber dem betrieblichen Management aus. Für eine solche Unterwanderung des Prinzips der gruppengetragenen Selbstorganisation steht ein Beispiel aus dem Betrieb PH. Hier hatte sich eine Gruppe im Gruppengespräch ein Fachthema überlegt, zu dem es einen Optimierungsvorschlag erarbeiten wollte. Es handelte sich um ein aufwendiges betriebliches Problem, von dessen Lösung sich die Betriebsleitung längerfristig eine nicht unbeträchtliche Summe an Einsparungen versprach. Die Gruppe investierte viel Zeit, Ehrgeiz und Engagement in das Projekt und präsentierte nach einigen Monaten ihre Ergebnisse vor Betriebsleitung, Betriebsmeistern und ihrem Schichtmeister. 
Dieser hatte sich aus dem Projekt völlig herausgehalten, als „stiller Beobachter“ jedoch sehr wohl die Diskussionen und Ergebnisse seiner Mannschaft verfolgt. Im Rahmen der Präsentation kam es dann zum Eklat. Nachdem die Gruppe ihren Vorschlag vorgestellt hatte, meldete sich der Schichtmeister zu Wort und zeigte in seinem Beitrag nicht nur die Fehler des Projektvorschlags auf, sondern präsentierte gleichzeitig einen Gegenvorschlag. Von der Gruppe wurde dies als demütigend empfunden, sahen sie sich doch vor der Betriebsleitung und den Meistern offiziell als „kleine Idioten“ hingestellt. Für den Schichtmeister hingegen waren die alten Machtverhältnisse wieder hergestellt und seine fachliche Überlegenheit gegenüber der Schicht bewiesen. Interessant ist dieser Fall vor allem deshalb, weil sich der Schichtmeister in allen Punkten an das Konzept gehalten hat, die Gruppe weder in ihrer Selbstorganisation eingeschränkt hat, noch die Gruppengespräche manipulierte. Der Schichtmeister wird in diesem Fall als „Innovationsbremse“ nicht aktiv tätig, sondern leistet in gewisser Weise „Dienst nach Vorschrift“. Die Weiterentwicklung der gruppengetragene Selbstorganisation wurde langfristig negativ beeinflusst. Die Selbständigkeit der Gruppe wurde, dies zeigen unsere Untersuchungen, nachhaltig beschädigt, und die Motivation, sich im Rahmen des KVP für den Betrieb zu engagieren, auf längere Zeit verspielt.

Dieses Verhalten ist auch den spezifischen Rahmenbedingungen der Produktion geschuldet. Denn solange die Verantwortung für die Schichtbelange beim Meister liegen muss, kann dieser sein Vorgehen durch äußere Rahmenbedingungen erklären und macht sich so bis zu einem gewissen Grad unabhängig von den in der Gruppe ausgehandelten Entscheidungen, eine auch aus Beschäftigtensicht problematische Konstellation.

„Die Machtposition des Schichtmeisters hat sich zum Teil noch verstärkt, denn wenn das Team etwas ausarbeitet, muss er es letztendlich absegnen...“ (Beschäftigter PH / 4)

Hier zeichnet sich ein aus den Untersuchungen zur Rolle der Meister im Reorganisationsprozess bekanntes Problem ab. Statusverluste werden vor allem durch „illegale“ Machtausübung (Bargmann 1984) unterlaufen. Das komplexe Regelwerk der Chemischen Industrie bietet ihnen dafür eine Fülle von Möglichkeiten.

Insgesamt wird das neue Profil von den Schichtmeistern positiv bewertet und mit einigem Engagement ausgefüllt. Unsere Ergebnisse zeigen, dass das Gros der befragten Schichtmeister bereits in einer neuen Rolle agiert, und eine Umprofilierung einmal in Bezug auf die Kooperation und Kommunikation mit der Tagschicht, d.h. dem betrieblichen Management, indirekten Bereichen und den Betriebsmeistern realisiert ist. Problematisch erweist sich das Anforderungsprofil allerdings für den Typus des älteren Schichtführers ohne IHK-Abschluss. Diese begegnen dem Projekt sehr zurückhaltend und sehen darin, folgt man unseren Auswertungen, vor allem eine Bedrohung ihres betrieblichen Status und ihres Arbeitsplatzes.

„Man hat den richtigen Eingriff nicht mehr, die verselbständigen sich. Ob jemand Schichtmeister oder Betriebsmeister ist, dass interessiert ja heute niemanden mehr. Durch Gruppenarbeit sind auf einmal alle wichtig, meinen sie zumindest.“ (Schichtmeister PH/3)

Im Selbstverständnis dieser Schichtführer bleibt die Schicht die soziale Bezugsgruppe, die zusammen arbeitet und klar unterhalb des betrieblichen Managements steht. Für sie spielt der Schichtzusammenhang in seiner sozialen Dimension eine wichtige Rolle und die An- 
sätze von Teamarbeit erscheinen ihnen vor allem als Störgröße, die die aus ihrer Perspektive gute Zusammenarbeit behindert.

„Für mich ist dieser ganze Prozess eine Art Abnabelung. Ich kriege da immer weniger mit und das alles bringt nur die Schichten und den Meister auseinander.“ (Schichtmeister $\mathrm{AI} / 3)$

Neue Aufgaben wie Mitarbeit bei betrieblicher Planung interessieren sie nicht oder kaum. Sie sind nicht in der Lage, Impulse für eine Teamselbstorganisation in die Mannschaften zu geben. In ihren Schichten ist die Stimmung in Bezug auf Teamarbeit gedämpft: Es wird nicht klar, wo das Neue über die alte Arbeitsorganisation hinausgeht. Hier wirkt deutlich die Sozialisation im „klassischen“ Chemiebetriebs nach, wie wir sie in Kapitel zwei skizziert haben und unterstreicht die Tatsache, dass die Schichtführer keine Vorgesetztenfunktion im Sinn des klassischen Profils der Industriemeisters erfüllten. Ihre zeitliche, räumliche und fachliche Nähe zur Schicht brachte sie nicht in den Konflikt der „man in the middle“. Ihr Platz war wesentlich eindeutiger auf Seiten der Produktionsmannschaften. Erst durch das steigende Qualifikationsniveau innerhalb der Schichten und die wachsende Zahl der Schichtführer mit IHK-Ausbildung wandelten sich im letzten Jahrzehnt die Qualifikationsstrukturen auf dem Shop floor. Das Unterlegenheitsgefühl der Schichtführer basiert auf einem realen fachlichen Kompetenzdefizit. Zum einen sind die hierarchisch unterstellten Kollegen zunehmend besser qualifiziert. Das uneingeschränkte Primat des Erfahrungswissens, welches für die Phase der Branche als Un- und Angelerntenindustrie galt, ist damit immer weniger wirkungsmächtig. Zum anderen haben aber auch die auf gleicher Position stehenden Kollegen inzwischen mehrheitlich die Meisterschule absolviert. Zwar ist ein direkter Vergleich durch die zeitversetzten Schichten nicht wirklich, dennoch manifestiert sich auch im Chemiebetrieb die fachliche Einschätzung eines Vorgesetzten und die daraus resultierende Sachautorität über den Kontrast mit anderen Positionsinhabern.

„Nichts mindert die Sachautorität eines Vorgesetzten mehr als die gleichzeitige Präsenz anderer, ihm formal gleichgestellter Vorgesetzter, die ihm fachlich überlegen sind. Die höhere Qualifikation der anderen setzt einen Autoritätsstandard, an dem auch er gemessen wird und dessen Unterschreiten von den Untergebenen um so schärfer akzentuiert wird.“ (Sofsky, Paris1994:52)

Berücksichtigt man diese Entwicklung, so lässt sich die Abwehrhaltung der Schichtführer nicht als eine irrationale Angst vor einem Statusverlust interpretieren, sondern als durchaus realistische und vernünftige Abschätzung des im Organisationswandel für sie enthaltenen Risikos, von den neuen Anforderungen überfordert zu sein. Da sich die Unternehmen der Chemischen Industrie jedoch für die Einführung einer „echten“ Meisterposition auf Ebene der Schichten entschieden haben, dürfte dieser Typus als Übergangsphänomen gewertet werden: Ernannte Meister wird es in Chemiebetrieben langfristig nicht mehr geben und wohl auch nicht mehr geben können. Für die Schichtmannschaften, die sich mit diesem Typus konfrontiert sehen, kann dies heißen, dass sie weiterhin von schichtübergreifenden Aufgaben und den Möglichkeiten zum Innovationshandeln ausgeschlossen bleiben.

Wie wichtig diese Modifikation der Rolle des Schichtmeisters für die gruppengetragene Selbstorganisation ist, unterstreicht ein Vergleich der Befragungsergebnisse und zwar 
zwischen einzelnen Schichtgruppen in den Betrieben PH und AI. In den Schichten, in denen die Schichtmeister dem neuen Anforderungsprofil bereits entsprechen, fallen die Befragungsergebnisse in den Dimensionen von Arbeitszufriedenheit, der Veränderung der Zusammenarbeit mit den Vorgesetzten und dem Ärger mit den Vorgesetzten insgesamt positiver aus, als in den Schichten, die noch mit dem Schichtführer alter Prägung konfrontiert sind.

\begin{tabular}{|l|l|l|l|}
\hline \multirow{4}{*}{ Arbeitszufriedenheit } & & neue Rolle & unverändert \\
\cline { 2 - 4 } & zufrieden & 70 & 55 \\
\cline { 2 - 4 } & unentschieden & 27 & 35 \\
\cline { 2 - 4 } & unzufrieden & 3 & 10 \\
\hline \multirow{3}{*}{$\begin{array}{l}\text { Zusammenarbeit mit } \\
\text { Vorgesetzten }\end{array}$} & verbessert & 40 & 21 \\
\cline { 2 - 4 } & unverändert & 53 & 65 \\
\cline { 2 - 4 } & verschlechtert & 7 & 15 \\
\hline \multirow{2}{*}{$\begin{array}{l}\text { Ärger mit } \\
\text { Vorgesetzten }\end{array}$} & wenig & 47 & 34 \\
\cline { 2 - 4 } & mittel & 41 & 32 \\
\cline { 2 - 4 } & stark & 13 & \\
\hline
\end{tabular}

Tabelle 8: Arbeitssituation und Rolle des Schichtmeisters (I)

Auch in der Beurteilung der Stellung des Teams zum Schichtmeister zeigt sich: Wo der Schichtmeister die Gruppen in ihrer Selbstorganisation unterstützt, relevante Fragen mit ihnen erörtert und vor allem auch die Interessen der Teams gegenüber dem Management vertritt, wird dies von den Beschäftigten durchweg positiv bewertet.

\begin{tabular}{|l|l|l|l|}
\hline \multicolumn{2}{|l|}{ Der Schichtmeister... } & \multicolumn{2}{|l|}{} \\
\hline & \multicolumn{2}{|l|}{ neue Rolle } & unverändert \\
\hline \multirow{2}{*}{$\begin{array}{l}\text { lässt das Team Probleme } \\
\text { selbständig lösen }\end{array}$} & Ja & 73 & 45 \\
\cline { 2 - 4 } & teils, teils & 23 & 31 \\
\cline { 2 - 4 } & Nein & 4 & 24 \\
\hline \multirow{2}{*}{$\begin{array}{l}\text { vertritt die } \\
\text { Interessen des }\end{array}$} & Ja & 50 & 27 \\
\cline { 2 - 4 } $\begin{array}{l}\text { Teams nach außen } \\
\text { spricht sich in } \\
\text { wichtigen Fragen mit } \\
\text { dem Team ab }\end{array}$ & Neils, teils & 30 & 31 \\
\cline { 2 - 4 } & Ja & 20 & 42 \\
\cline { 2 - 4 } & teils, teils & 50 & 28 \\
\cline { 2 - 4 } & Nein & 33 & 36 \\
\hline
\end{tabular}

Tabelle 9: Arbeitssituation und Rolle des Schichtmeisters (II)

Für die Mehrheit der Schicht- und Betriebsmeister gilt, dass sie sich ohne Bedenken auf die ihnen angebotene Herausforderung einlassen können, denn wo sich in den Fertigungsindustrien auf der Ebene der Meister oftmals das „betriebspolitische Dilemma der Dezentralisierungsprojekte“ (Faust et al. 1995) abzeichnet, scheint das Spiel für die Akteure in 
der Chemischen Industrie aufzugehen: Die Demontage der eigenen alten Position hat eine aufgewertete neue zur Folge. Wenig spricht hier für eine neue Meisterkrise, vieles spricht für eine Stärkung der Führungsrolle mit neuem Anforderungsprofil. ${ }^{178}$ Dass die Position des Obermeisters als mittel- bis langfristige Aufstiegsoption für die Betriebsmeister obsolet wird, scheint zumindest in unserem Sample durch die dezidierte Aufwertung der Meisterfunktion kein Problem zu sein. Schwerer wiegt hingegen, dass eine quantitative Reduktion der Meisterstellen sowohl die Aufstiegsmöglichkeiten für die Schichtmeister reduziert, als auch die Exit-Optionen aus der Schichtarbeit verringert - eine Tatsache, die für viele Schichtmeister weit mehr Gewicht hat, als der innerbetriebliche Aufstieg.

\subsection{Fazit: Nicht nur die Chemie muss stimmen...}

Unsere Befunde zeigen: Im Hinblick auf die Gestaltungsziele sind die Ausgangsbedingungen für das Projekt zur prozessorientierten Fertigung im Team recht günstig, und vor allem im Bereich der Aufgaben- und Funktionserweiterung sind in der Vergangenheit bereits viele Konzeptideen - wenn auch unsystematisch und informell - umgesetzt. Allerdings beinhaltet die im wesentlichen von nicht-tayloristischen, teamartig-kooperativen Merkmalen geprägte Arbeitssituation aus Perspektive der Beschäftigten wenig Veränderungsbedarf. Dies zeigt sich insbesondere bei der Frage nach den Gesamtveränderungen durch die Einführung selbstorganisierter Gruppenarbeit, die selbst bei hohem Realisierungsniveau noch von fast 60 Prozent der Befragten als unverändert bewertet wird. Die für die technisierten Produktionsbereiche im allgemeinen wie auch für die Chemische Industrie im besonderen immer wieder konstatierte deutlich weniger von traditionelltayloristischen Arbeitsbelastungen geprägte Arbeitssituation spielt bei diesem Urteil eine entscheidende Rolle. Die Reorganisation in der Fertigungsindustrie gewinnt ihre Unterstützung bei den Mannschaften insbesondere in ihrer Zielsetzung, gleichsam Verlorenes wieder herzustellen und negative Arbeitsfolgen tayloristischer Rationalisierung zu korrigieren. Die durch detail- und sekundengenaue Arbeitsplanung eingeengten Verhaltensspielräume und Qualifikationsnachfragen werden durch die innovative Arbeitspolitik merkbar wieder erweitert, und dieser Surplus ist für die Mannschaften evident. Demgegenüber verlief die Entwicklung in der Chemie genau umgekehrt, wir zeigten dies ausführlich in Kapitel 2. Ausgangspunkt ist hier eine von Haus aus verwissenschaftlichte Fertigung, bei der das Produktions-Know-how einseitig bei der Betriebsleitung lag. Die Beschäftigten leisteten zunächst lange Zeit kaum mehr als Zuarbeiten. Dieser Zustand wandelte sich in den letzten 20 bis 30 Jahren recht grundlegend, mit einem auch wissenschaftlich-technisch induzierten Schub Ende der 1980er, Anfang der 1990er Jahre. Die wachsende Prozess- und Anlagenkomplexität, die neuen Steuerungstechniken und die erhöhten Output-Ansprüche erfordern nun auch auf dem Shop-Floor zunehmend mehr professionalisierte Arbeit. In der Regel bewerten deshalb Beschäftigte in technisierten Bereichen die Arbeitssituation bei gleichem Realisierungsniveau die Arbeitssituation signifikant schlechter als Vergleichsgruppen in den Handarbeitsbereichen. ${ }^{179}$

Reorganisationsprojekte gewinnen aber immer dann ein hohes Maß an Unterstützung, wenn es den Promotoren des Konzepts gelingt, die bestehenden Strukturen, die es zu ver-

\footnotetext{
178 Jauch, Faust 1998, Jauch 1999, Kädtler, Hardwig 1998.

179 Gerst et al. 1995; Schumann, Gerst 1997; Gerst 1998; Kocyba, Vormbusch 2000.
} 
ändern gilt, negativ zu konnotieren und für die Zukunft Verbesserungen zu versprechen (Luhmann 2000). Das Problem der Chemischen Industrie ist, dass das „Vergangene“ nicht so belastend ist, wie etwa die Arbeitssituation in Bereichen der Handmontage der Fertigungsindustrie. Dies belegten schon die Ergebnisse des Trendreport Rationalisierung. Die AutorInnen kommen trotz der durchaus gravierenden objektiven Unterschiede in punkto Qualifikationsaufwendungen, -erwerb und -entfaltung in den betrieblichen Organisationstypen (traditionell-hierarchisch, flexibel-teilintegriert, flexibel-hochintegriert) zu dem Ergebnis, dass bei allen befragten Regulierern eine positive Einschätzung der Arbeitssituation überwiegt. Die Arbeit wird von der Mehrheit als interessant wahrgenommen, und auch im Hinblick auf Aspekte der Selbständigkeit, fachlich-inhaltliche und berufliche Dimensionen schneidet die Arbeitssituation in der subjektiven Bewertung der Befragten sehr positiv ab. Insbesondere die Produktionsmodernisierung der 1980er Jahre hat hier für das Gros der Beschäftigten eine Verlagerung der Tätigkeitsschwerpunkte mit sich gebracht, und so sind eine Reihe körperlich belastender Vorgänge (manuelle Umstellungen oder Stoffzugabe) entfallen (Schumann et al. 1994). Insgesamt ist festzustellen, dass durch das hohe Niveau, auf dem die Beschäftigten in der Chemischen Industrie in Bezug auf die Arbeitsorganisation starten, deutlich höhere Anforderungen an die Einführung selbstorganisierter Gruppenarbeit gestellt werden, damit diese als Verbesserung bzw. überhaupt als Veränderung der Arbeitssituation wahrgenommen wird.

Da also Produktionsarbeit in der Chemischen Industrie wegen ihres technischen Niveaus bereits per se einen erhöhten Anteil an technologiebezogenen indirekten Aufgaben beinhaltet, koordiniertes Arbeiten erlaubt, intensive Zusammenarbeit sowie die Suche nach gemeinsamen Problemlösungen fördert und fordert, weist sie in vielen Elementen eine hohe Ähnlichkeit mit der im Konzept festgelegten neuen Arbeitsform auf und es finden sich unterstützende Anknüpfungspunkte für innovative Arbeitspolitik. Allerdings ist diese förderliche Ausgangssituation keinesfalls dazu geeignet, aus arbeitspolitischen Projekten einen Selbstläufer zu machen.

Im Gegenteil: Stärker noch als in anderen Branchen bedarf es eines integrierten Reorganisationsansatzes, der sowohl die Arbeits- wie die Betriebsorganisation einbezieht und Wissensbestände wie Kompetenzen in neuartiger Weise miteinander verschränkt. So definierte Gestaltungsansätze greifen weitreichend in die betrieblichen Strukturen ein. Die Beharrungskräfte der betrieblichen Sozialorganisation können sich in diesem Kontext als besonderes Hemmnis erweisen. Die Bereitschaft zu und die Motivation für die Umstellung von der funktions- auf eine prozessorientierte Arbeits- und Betrieborganisation werden damit zum wichtigen Einflussfaktor. Wir haben am Beispiel der Fälle AI und PH zeigen können, dass dieser Wandel der Praxis in Betrieben der Chemischen Industrie dann in Gang zu setzen ist, wenn es zu einer umfassenden Entwicklung und Verlagerung von Kompetenzen der betrieblichen Akteure kommt. Dieser Wandel wird forciert durch die uneingeschränkte Unterstützung des Managements, das Prozessorientierung immer auch als Teamorientierung definiert. Darüber hinaus spielen aber auch die Markt-, Produktund Innovationsanforderungen, denen sich die Betriebe stellen müssen, eine Rolle. In dem Maß, in dem die Innovationsaffinität stark ausgeprägt ist, steigen die Anforderungen an das betriebliche Innovationshandeln. Die Betriebsleitung verlagert Kompetenzen und Verantwortung, und für alle betrieblichen Ebenen erweitert sich die Funktionsmasse zur Definition qualifikationsadäquater Anforderungsprofile. Das starke Commitement des betrieblichen Managements ist damit auch Ausdruck der Überzeugung, dass mit den „här- 
teren“ Arbeitsanforderungen auf allen Ebenen auch eine „intelligentere“ Arbeits- und Betriebsorganisation korrespondieren muss.

Für die betrieblichen Strukturen heißt dies, dass erstens das fach- und aufgabenzentrierte Kompetenzmodell der funktionsorientierten Organisation in Richtung eines prozessbezogenen redefiniert wird. Die Spezialisierung entlang berufstypischer Qualifikationen im Bereich der Instandhaltung weicht einer prozessorientierten Auflockerung der berufstypischen Einsatzkonzepte und Aufgabenprofile, die Anforderungsprofile der Chemikanten werden facharbeitsspezifischer. Funktionsübergreifende und integrierende Prozesse der Kommunikation und Kooperation lösen tradierte und als dysfunktional definierte Muster der Sozialintegration (etwa den fachspezifisch exklusiven Einsatz oder institutionalisierte Karrierewege) mit erheblichen Konsequenzen für die Kompetenzprofile ab.

In der fachlichen Dimension hat dies eine heterogenere und prozessnähere Bestimmung dieser Profile zur Folge, die von einer Ausweitung sozial-kommunikativer Anforderungen ebenso wie der Fähigkeit zur Selbstorganisation im Arbeitshandeln flankiert wird. Dies bedeutet zweitens, dass das dem Prinzip des Dienstwegs verpflichtete Kooperationsmodell abgelöst wird von direkter Kommunikation und Kooperation und zwar sowohl in der horizontalen wie auch vertikalen, also hierarchieübergreifenden Richtung. Die betrieblichen Strukturen verändern sich damit gegenüber der funktionsorientierten Struktur erheblich, wie folgende Abbildung verdeutlicht. 


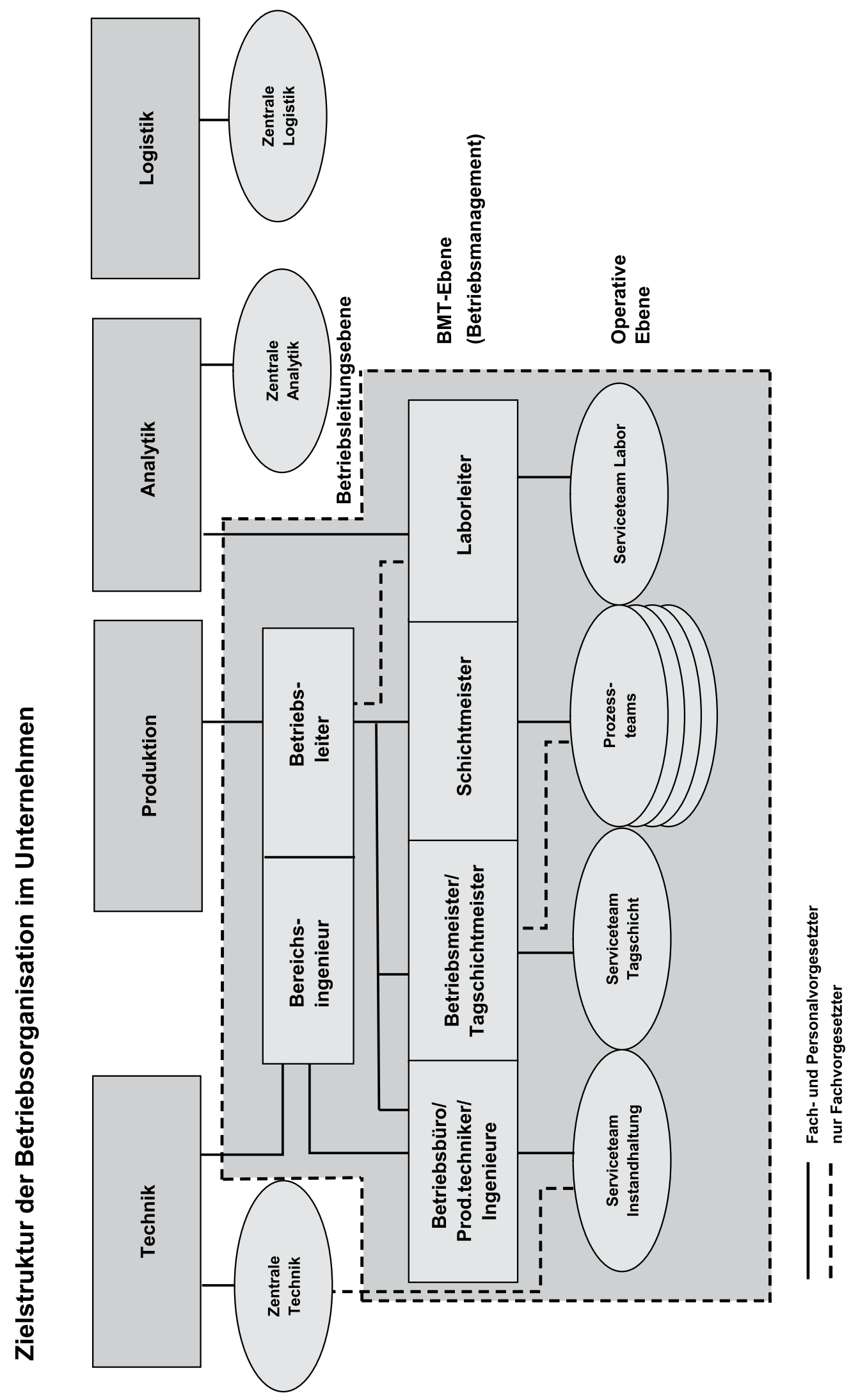

Abbildung 14: Zielstruktur der Betriebsorganisation des Unternehmens; SOFI (2003) 
Und schließlich gilt drittens für das Statusmodell, dass Hierarchien und Aufstiegspositionen abgebaut werden und formale Kompetenzen eine geringere Rolle spielen. Der Zusammenhang exklusiver Zuordnung von Innovationskompetenz, hierarchisch geprägter Koordination und hochgradiger Statusdifferenzierung löst sich auf (Baethge, BaethgeKinsky 1998). Die offizialisierte Anerkennung der spezifischen Wissensbestände der Beschäftigten durch ihre Einbindung in die betriebliche Organisation im Rahmen von KVP und veränderter Kooperations- und Kommunikationsstrukturen beinhaltet für die Entwicklung von Chemiearbeit eine fachlich-soziale Aufwertung auch auf dem Shop floor, auch und gerade gegenüber anderen Berufsgruppen.

Die berufliche Kompetenz bildet in diesem Modell eine gewichtige Grundlage für eine umfassende Aufgabenintegration und weitreichende Kooperationsbezüge - und macht diese dadurch erst möglich. Andererseits kann diese zentrale Stärke, die der Berufsausbildung dabei zukommt, auch negativ wirken, stellt sie doch nach wie vor das Bezugssystem für ein kategoriales Denken und damit verbundene Prioritätensetzungen im Arbeitshandeln. Dass die berufsfachlich geprägten Arbeits- und Ausbildungskonzepte als „Innovationsbarrieren“ und „Bremsklotz“ (Baethge 1996; Kern, Sabel 1994) bei der Umsetzung dieser teamartigen Kooperationsformen wirken können und der Stärkung prozessbezogener, bereichs- und fachübergreifender Kompetenzen entgegenstehen, wurde in den 1990er Jahre nicht nur in den Sozialwissenschaften diskutiert. ${ }^{180}$ In dem Maß, in dem sich bei der Umstellung auf prozessorientierte Abläufe die Dienstleistungsfunktionen, also die Instandhaltung, unter das Primat der Produktion zu unterwerfen haben, muss diese Einschätzung für die Entwicklung in der Chemische Industrie differenziert werden. Für die direkte Produktion ist erstmals eine Situation bezeichnet, in der Chemikanten selbstbewusst und autonom neben ihren Kollegen aus der Instandhaltung auftreten können. Diese berufsfachliche Stärkung der direkten Produktion ist, wir haben dies anhand unseres Materials zeigen können, in der Kombination mit selbstorganisierter Gruppenarbeit weit davon entfernt, zum Bremsklotz von Innovation zu geraten. Im Gegenteil: Die Beschäftigten in diesen Bereichen haben erstmals die Chance, gleichsam ,auf Augenhöhe“ mit ihren Facharbeiter-Kollegen im betrieblichen Status-, Kooperations- und Kompetenzmodell zu agieren und aktiv auf Basis ihrer Qualifikationen und Kompetenzen in das Innovationsgeschehen einzugreifen. Die Facharbeiter der indirekten Bereiche werden im Rahmen des von uns untersuchten Projekts deutlich näher an der Produktion angesiedelt, und ihre organisatorische wie fachliche Anbindung an die Zentralwerkstätten wird geringer. Die berufsbezogenen Bornierungsgefahren sind damit zwar abgemildert, aber gleichermaßen sinken die (berufs-) fachlichen Identifikationsangebote. Wie sich die Facharbeiter in diesen Bereichen in Zukunft orientieren werden bzw. können, wird zu beobachten sein. Allerdings bietet die neue prozessorientierte Arbeitsorganisation einige Andockpunkte für die Herausbildung einer neu definierten Berufsfachlichkeit, zu der eben auch die fachübergreifende, kollektive Problemlösung zählt (Schumann 2002).

\footnotetext{
${ }^{180}$ Vor allem drei Kritikpunkte an den geltenden Regelungen der Erstausbildung wurden in den 1990er Jahren auch seitens der Großindustrie immer häufiger formuliert (Hofmann, Röben 1997). Erstens sollte die durch ihre Ferne zum (einzelbetrieblichen) Arbeitsprozess zu stark auf abstrakte Tätigkeiten und Grundfertigkeiten zielende Ausbildung betriebnäher formuliert werden, denn noch immer schienen den Unternehmen die Einarbeitungszeiten nach Abschluss der Erstausbildung zu lang. Außerdem sollte zweitens die im Rahmen von Maßnahmen zur Prozessoptimierung immer relevantere „Problemlösefähigkeit“, d.h. die Fähigkeit, technische Artefakte in ihrem Nutzen für die Produktion zu kritisieren, und Alternativen zu entwerfen, stärker gefördert werden. Und drittens schließlich erschwere die nach Berufsfeldern getrennten Ausbildungen die fachübergreifende Kooperation und Kommunikation, da sie Berufsegoismen verstärke.
} 
In den elaborierten Fällen kommt es zu einer eindeutigen Abkehr vom AngelerntenModell und damit steigen die Chancen für eine Aufwertung der Produktionsarbeit in Richtung Facharbeit für das Gros der Beschäftigten. Die Handlungssituation der Beschäftigten ist zunehmend gekennzeichnet durch das Zusammenspiel komplexer und einfacher Regulierungshandlungen zur Sicherung wie Wiederherstellung der Prozessstabilität. Diese Eingriffskompetenzen sind auf die gesamte Schicht ausgeweitet, die als Team alle Aufgaben offiziell eigenständig erledigt. Die schichtinterne Hierarchie ist aufgelöst, und auch bereichsorganisatorische Grenzen werden durchlässiger. Die Kooperation mit Spezialisten und Instandhaltern zur Lösung von Anlagenprobleme gehört zum erweiterten Anforderungsprofil, die Beschäftigten in der Produktion werden als „Experten der Praxis“ zu gleichberechtigten Gesprächs- und Kooperationspartnern. Eine besondere Rolle kommt in diesen Fällen der Prozessoptimierung zu. ${ }^{181}$ Im Gegensatz zu den Fällen auf niedrigem Realisierungsniveau erschöpft sich diese nicht allein in Teaminitiativen zum KVP, sondern erhält wichtige Impulse durch sowohl vom betrieblichen Management initiierte Themen wie auch von den Schichtmannschaften und dem Management gemeinsam vereinbarten Problemfeldern. Zugleich steht die in diesen Fällen praktizierte Form der Prozessoptimierung in besonderer Weise für eine Reorganisation der betrieblichen Strukturen.

Dass diese Einbindung der Beschäftigten in die Prozessoptimierung nicht auf einen „arbeitspolitischen Konservatismus“ (Schumann 1982) der Schichtmannschaften stößt, ist, dies belegen unsere Befunde, auch in der Chemischen Industrie in besonderer Weise dem Konzept der selbstorganisierter Gruppenarbeit geschuldet. Denn inwieweit sich die Beschäftigten auf einen „New Deal“ (Dörre et al. 1993) einlassen, ist in hohem Maß davon anhängig, welche Möglichkeiten den Prozessteams zur Verbesserung der Arbeitssituation und damit zur Belastungsregulation geboten werden. Ein deutlicher qualitativer Unterschied zur Arbeitsgestaltung im flexibel-hochintegrierten Organisationstyp besteht deshalb erstens darin, dass sich Optimierung im Konzept selbstorganisierter Gruppenarbeit nicht allein auf Fragestellungen reduziert, die zur Steigerung der Wirtschaftlichkeit, also zur Bewältigung von betrieblichen Anforderungen beitragen. Als gleichrangig werden auch für die Beschäftigtenbedürfnisse relevante Themenkomplexe zum Gegenstand des KVP. Damit ist ein zweites Unterscheidungskriterium bereits angedeutet. Die institutionelle Fundierung von Gruppengesprächen und Gruppensprechern wird für die Schichtmannschaften zur wichtigen Ressource, um individuelle wie gruppenspezifische Möglichkeiten zur Belastungsregulation auszuhandeln. Selbstorganisierte Gruppenarbeit auf hohem Realisierungsniveau hat damit auch in der Chemischen Industrie das Potential, ein kooperatives Gruppenklima zu forcieren.

Ob sie das Spannungsverhältnis zwischen betrieblichen Anforderungen, individuellen Interessen und Teamvorstellungen nachhaltig zugunsten der Beschäftigten auflösen kann, bleibt fraglich. So ergeben die Befunde des SOFI-Projekts „Konzepte innovativer Arbeitspolitik“ für den Betrieb PH rund fünf Jahre nach unseren Untersuchungen ein deutlich negativeres Bild der subjektiven Bewertung der Arbeitssituation durch die Beschäftigten. Hintergrund dafür sind sowohl im Hinblick auf Markt-, Produkt- und Innovations-

\footnotetext{
${ }^{181}$ Zu diesem Ergebnis kommt auch der Schlussbericht „Konzepte innovativer Arbeitspolitik“ des SOFI (Balzert et al. 2003).
} 
anforderungen wie auch auf die Unterstützung durch das betriebliche Management gewichtige Veränderungen, die sich auf die Realisierung selbstorganisierter Gruppenarbeit negativ auswirken. Dies manifestiert sich einmal in der personellen Besetzung: Auf allen betrieblichen Ebenen wurden Know-how-Träger zum Aufbau eines neuen Betriebs an einen anderen Standort versetzt. Neben dem Verlust an Erfahrungswissen hat der Betrieb damit zugleich wichtige Protagonisten des Projekts verloren. Darüber hinaus wurden Produkt- wie Prozessoptimierungen im Hinblick auf Ausbeute und Qualität durch das hohe Engagement Mitte bis Ende der 1990er Jahre nahezu ausgereizt. Der Schwerpunkt liegt nun auf der Mengenproduktion, und die Beschäftigten sind einem hohen Produktionsdruck ausgesetzt. Entsprechend wenig Raum bleibt für Prozessoptimierung, die Einbindung in das betriebliche Innovationsgeschehen findet kaum noch statt. An den Ergebnissen lässt sich der Unmut der Beschäftigten über diese Veränderungen ablesen, und es bestätigen sich Befunde aus den SOFI- Gruppenarbeitsuntersuchungen der 1990er Jahre in der Fertigungsindustrie: ${ }^{182}$

Die positive Haltung der Beschäftitgen zur innovativen Arbeitspolitik, die sich in einem neuen betrieblichen Selbstverständnis auf der Grundlage einer aktiven Beteiligung an Rationalisierung niederschlägt, löst die grundsätzlichen Interessengegensätze um Lohn, Leistung und Beschäftigung der Produktionsarbeiter nicht auf. Die Beschäftigten bleiben kritische Beobachter der betrieblichen Arbeitspolitik, und die in Gang gesetzten Veränderungen des betrieblichen Status-, Kooperations- und Kompetenzmodells lassen sich nicht anhalten „wie ein Fließband“ (Dubiel 1997). Ob und welche Folgen die Rücknahme von Elementen selbstorganisierter Gruppenarbeit und die damit zusammenhängenden Enttäuschungen der Teams für die ökonomische Leistungsfähigkeit der Betriebe allerdings in Zeiten immer unsicherer wirtschaftlicher wie sozialer Rahmenbedingungen von Industriearbeit haben können, bedarf der weiteren empirischen Fundierung.

${ }^{182}$ Vgl. dazu Gerst, Schumann 1997; Schumann, Kuhlmann 2000. 


\section{Schlussbemerkungen}

Die Chemische Industrie gehört zu den klassischen Kernsektoren der deutschen Industrielandschaft, die im Gegensatz zu den metallverarbeitenden Branchen keine handwerklichen Vorläufer hatte, sondern als angewandte Wissenschaft entstand. Diese Tatsache hat entscheidend dazu beigetragen, dass der Produktionsbeschäftigte in der Chemischen Industrie über Jahrzehnte ganz überwiegend dem Typus des wenig qualifizierten Massenarbeiters entsprach und seine Tätigkeit eine restriktive, entfremdete war. Diese Aufgabenund Funktionskontur der Chemiearbeit erlebte Veränderungen vor allem durch die technologische Entwicklung, die in zunehmendem Maße kompensierende und korrigierende menschliche Eingriffe zur Applikation, Flexibilisierung und Kontrolle der technischen Systeme notwendig machte. Technisch vermittelt und nur teilweise arbeitsorganisatorisch gestützt, entstanden fachlich qualifiziertere Varianten der Chemiearbeit, die eine Rücknahme von Arbeitsteilung sowie die Erweiterung von Handlungsspielräumen und Kompetenzen erkennen ließen. Diese Aufwertung und breitere Nutzung lebendiger Arbeit blieb allerdings in Bezug auf die Selbstorganisationsmöglichkeiten und die Zuständigkeiten der Beschäftigten zu eng und für eine innerbetriebliche Emanzipation der Produktionsarbeiter entsprechend prekär. Denn die Arbeitskraftnutzung entwickelte sich auf Basis einer Arbeitsorganisation, die lediglich betrieblich isolierte, informelle Gestaltungsspielräume bot und zudem durch eine Betriebs- und Innovationsorganisation gestützt wurde, deren hierarchisch-arbeitsteilige Strukturen den Status lebendiger Arbeit beständig reproduzierten (Kap. 2).

Diese Konstellation hat sich in den neunziger Jahren grundlegend verändert: Die Chemieunternehmen vollziehen einen bis heute anhaltenden tief greifenden Strukturwandel, der die Unternehmenslandschaft erheblich verändert. Internationalisierung, Konzentration auf Kernprozesse und Investitionen in neue, wissensintensive Zukunftsfelder sind die strategischen Fluchtpunkte dieser Entwicklung, die sich in einer finanzmarkt- und wissensorientierten Restrukturierung mit weitreichenden Folgen für Formen und Inhalt von Arbeit widerspiegelt (Kap. 3).

Die finanzmarktorientierte Restrukturierung manifestiert sich in der Formulierung der strategischen Leitlinien der Unternehmen, deren zentraler Begriff das Shareholder-value wird, und die auf Anforderungen und Normen der globalen Finanzmärkte bezug nehmen (Kädtler 2003). Dies wirkt sich erstens auf die strategischen Produktionsentscheidungen aus. Die in der Triade traditionell seit langem international aufgestellten Chemieunternehmen weiten ihre Produktion nun auf die Regionen China und Südostasien aus und Wachstum findet durch die Expansion neu entstehender Märkte in der bisherigen Peripherie statt. Zugleich erfolgt zweitens die Definition des Geschäftsportfolios zunehmend im Hinblick auf die potentielle Renditeentwicklung. Die äußerst heterogene Zusammensetzung der Produktpaletten in der Chemie- und Pharmaindustrie, die sich historisch aus deren Wissenschaftsbasierung ergeben hat, bildet für diese Redefinition der Kernkompetenzen vielfältige Andockpunkte (Becker 2001; Dolata 1997).

Parallel dazu stellt das Zusammenwirken der informations- und biotechnologischen Entwicklungen die Branche vor erhebliche organisatorische und personelle Herausforderungen, und die für die Chemieunternehmen substantielle Generierung wie Integration wissenschaftlich-technologischen Wissens erfährt eine qualitativ wie quantitativ veränderte 
Bedeutung. Neues wissenschaftliches Wissen wird immer weniger allein innerhalb des herkömmlichen Wissenschaftssystems, sondern in vielen autoorganisierenden Räumen und zunehmend heterogeneren Formen der institutionellen, organisationalen und disziplinären Ordnung erzeugt. ${ }^{183}$ In immer kürzeren Zyklen muss immer mehr und immer heterogeneres Wissen bearbeitet werden bzw. sind veränderte Formen und Inhalte von Wissen zu lokalisieren und verfügbar zu machen. Die industrielle FuE kommt dabei eine doppelte Funktion zu. Zum einen gilt es, intern die Steuerung und Koordination der Wissenschafts- und Technologieentwicklung zu effektivieren, zum anderen ist die externe Wahrnehmung und Realisierung dieser Entwicklungen zu gewährleisten (Hack 1998).

Eine grundlegende Anpassung der Organisations- und Steuerungsformen der Wissensproduktion an diese Rahmenbedingungen ist zunehmend ausschlaggebend für ein dynamisches Innovationsgeschehen. Um an den Standorten präsent zu sein, an denen wichtige wissenschaftlich und technologische Entwicklungen getätigt werden, und darüber hinaus die besten „Köpfe“ direkt von den Hochschulen rekrutieren zu können, kommt es zu einer räumlichen Neuordnung der FuE, und die Internationalisierung der FuE-Aktivitäten nimmt zu (Hack 2002). Bestimmte Forschungslinien werden in diesem Zusammenhang aus den Konzernzentralen ausgelagert, substituiert oder ergänzt durch Kooperationen mit Kontraktforschungsunternehmen, staatlichen Forschungseinrichtungen oder Universitäten sowie kleinen Start-up-Unternehmen (Wittke, Buss 2001; Briken, Kurz 2003). Zugleich entstehen enger verflochtene, direkter aufeinander bezogene Formen der gemeinsamen Wissensproduktion von Wissenschaft und Industrie: 22 Prozent aller Chemieunternehmen sind in Forschungskooperationen mit Hochschulen aktiv, in der Industrie insgesamt sind dies nur knapp sieben Prozent (ZEW 2003).

Aus der science based industry wird so zu Beginn des 21. Jahrhunderts mehr und mehr eine knowledge based, also eine wissensbasierte Industrie. Für den Innovationsprozess werden Wissensformen relevant, denen im geläufigen Verständnis wissenschaftlichen Wissens keine Bedeutung zugewiesen wurde, wie etwa intuitives Wissen und Erfahrungswissen (Hack 1998). Zusätzlich zu Allgemeinbildung und explizitem Wissen wird personen- und organisationsgebundenes implizites Wissen systematischer in den Innovationsprozess eingebunden. Die unternehmerische „Kompetenz zur Innovation“ (Staudt 1997) erschließt sich zunehmend aus der Verknüpfung unterschiedlicher Wissensbestände.

Als eine adäquate Umsetzung der Verbindung technologischer und organisatorischer Umgestaltungen mit neuen Strategien der Kapitalverwertung hat sich in den Großunternehmen die prozessorientierte Ausrichtung der Betriebs- und Arbeitsorganisation durchgesetzt. Diese Prozessorientierung und die Fokussierung auf konsequente Kostensenkungsstrategien stützen neue Organisationsformen von Arbeit und Ausbildung in der Chemie.

\footnotetext{
${ }^{183}$ Dies ist eine der Grundannahmen der Wissensproduktion im von Gibbons et al. (1994) als „Mode 2“ bezeichneten System der Wissensproduktion, dass den Mode 1, d.h. den für die Chemische Industrie in Kapitel 2 charakteristischen Prozess der Wissensproduktion ergänzt (vgl. zur Debatte Bender 2001; Nowotny 1999; Nowotny et al. 2001; Weingart 2001).
} 
Für die Ausschöpfung der kreativen und produktiven Potentiale der Beschäftigten in der direkten Produktion wird eine neue Phase einläutet. Optimierendes, selbstorganisiertes Arbeitshandeln verschränkt sich zunehmend mit dezentralen, prozessorientierten Formen der Betriebs- und Innovationsorganisation. Insgesamt sind von daher in der Branche die Voraussetzungen für eine auf innovativer Arbeitspolitik basierende Variante von Arbeitsgestaltung gut.

Unsere Ergebnisse zeigen, dass unter den Voraussetzungen innovativer Arbeitspolitik eine systematischere, schnellere und effektivere Nutzung, Mobilisierung und Integration von Qualifikationen und Kompetenzen der Beschäftigten stattfindet. Technische, funktionale und betriebliche Grenzen werden verschoben, und Ressourcen und Potentiale der betrieblichen Akteure neu verknüpft und gesteuert. Direkte, detailgenaue und breitflächig in festen Formen geltende Begrenzungen von Arbeitsprozessen lösen sich in den Produktionsteams zunehmend auf und werden auf einer systemisch „höheren“ Ebene von einer neuen strukturellen Steuerung ersetzt (Voß, Pongratz 1998). Insgesamt ist ein Wandel des tradierten Status-, Kooperations- und Kompetenzmodells in Gang gekommen. Dieses positive Bild der Effekte innovativer Arbeitspolitik bedarf sowohl hinsichtlich des Verbreitungsgrads als auch der Tiefe und Reichweite der erzielten Arbeitsveränderungen der Differenzierung.

Die innovative Logik kollektiver Problemlösungen erweist sich als kompatibel mit den Entwicklungstendenzen chemisch-industrieller Produktions- und Innovationsaktivitäten. Freilich müssen wir im Hinblick auf die vorliegenden Befunde auch konstatieren: Obwohl in kaum einer Branche die Möglichkeiten, neue Ideen auszuprobieren und sich im Innovationsprozess zu engagieren, so gut sind wie in der Chemischen Industrie, fassen die Produktionsbeschäftigten als Co-Akteure im Innovationsprozess nur langsam Fuß. Die Wirkungskraft des traditionellen, sich in einem über hundert Jahre andauernden Prozess herausgebildeten Innovationsmodells ist nicht über nacht obsolet geworden, sondern strahlt weiter kräftig in die betriebliche Realität hinein. Für die Produktionsarbeiter sind- arbeitssoziologisch gesprochen - ein großes Maß an Funktionsmasse und innovativem Aufgabenpotential im Spiel. Um diese ausschöpfen zu können, muss die Formel „Innovation als Querschnittsaufgabe“, will sie mehr sein als leere Floskel, Organisationsformen von Arbeit, die das Innovationspotential auf dem Shop floor stärken, systematisch berücksichtigen. Dass es gute Gründe auch für das Management geben kann, auf Funktionserweiterung und Selbststeuerung zu setzen, ist ein zentraler Befund dieser Arbeit. Die Prozessorganisation jedenfalls erweitert auch in der Chemie die Potentiale zur Arbeitsgestaltungwie sie zukünftig genutzt werden, bleibt abzuwarten. Fassen wir einige Trends zusammen.

Erstens: Der Durchdringungsgrad der Unternehmen mit innovativen Arbeitsgestaltungskonzepten ist bislang gering geblieben. Gruppenarbeit als neue Arbeitsform wurde zwar Mitte der neunziger Jahre auch in der Chemischen Industrie zum Thema, allerdings war dies nicht der Auftakt für eine breitflächige Umsetzung neuer Arbeitsformen in der Produktion. ${ }^{184}$ Die im Ergebnis recht schwache Resonanz, die Gruppenarbeit im weiteren

\footnotetext{
${ }^{184}$ Die folgenden Überlegungen nehmen Teile der Argumentation eines Vortrags von C. Kurz auf, der im Rahmen der Roche-Betriebsrätekonferenz „Zukunft der Arbeit, neues Wissen, neue Zeiten, neue Jobs“ am 24. Juni in Weinheim gehalten wurde.
} 
Verlauf der neunziger Jahre erfahren hat, dürfte vor allem damit zusammenhängen, dass die Arbeit schon seit langem mit teamförmigen Strukturen einhergeht. Das Arbeitshandeln der Schichten ist zwar einerseits durch enge Vorschriften, ein hohes Maß an Formalisierung und ein fein abgestuftes System der Betriebshierarchie gekennzeichnet. Anderseits bieten sich den Schichten aber - und deutlich anders als im Metallbereich - hohe Dispositionsspielräume und vielfältige Kooperationsmöglichkeiten. Der Bedarf, diese Aufgaben systematisch in die Produktionsteams zu integrieren und durch innovativen Arbeitsformen zu stärken, ist aber aus Perspektive der Akteure vor Ort so dringlich nicht, und es dominieren die tradierten Arbeitsteilungsmuster. In der Praxis hat dies zur Folge, dass das Anforderungsniveau der Arbeit dem fachlichen Ausbildungsniveau der Beschäftigten vielfach nicht mehr entspricht. Die verschärften Umwelt- und Sicherheitsbestimmungen lassen es zwar ratsam erscheinen, die Un- und Angelernte durch Chemiekanten zu ersetzen. Fachlich herausfordernde Arbeitszuschnitte sind auf diesem Weg aber nicht zu realisieren.

Zweitens haben die Ergebnisse unseres Pilotprojektes gezeigt, dass eine innovative Arbeitsgestaltung und damit einhergehende fachliche Aufwertung der Arbeit möglich und im Beschäftigteninteresse auch erfolgversprechend ist. Wir müssen indes einschränkend hinzufügen: Nicht jede Umsetzung der Aufgabenerweiterung, die im Gewand neuer Arbeitsformen erscheint, führt zu durchgreifenden Qualifizierungs- und Emanzipationseffekten in der Arbeit. Die Durchsetzungsbedingungen und Durchsetzungsmöglichkeiten innovativer Arbeitspolitik hängen entscheidend davon ab, ob und wie weit der kohärente Umbau von Arbeits-, Betriebs- und Innovationsorganisation gelingt (Kuhlmann et al. 2004). Für die Beschäftigten positive Effekte sind vor allem dann zu erzielen, wenn sich Aufgaben- und Funktionsintegration mit Gruppenselbstorganisation, vor allem aber einer erweiterten Einbindung der Produktionsgruppen in betriebliche Planungen und Fragen der Prozessoptimierung verbindet. Ausschlaggebend für die Reichweite der Arbeitseffekte ist, dass der arbeitsorganisatorische Wandel mit weitgehenden Veränderungen der Betriebsund Innovationsorganisation kombiniert wird. Die wichtigsten Ansatzpunkte neben einer Verlagerung der Aufgaben aus Zentral- und Servicebereichen in die Produktionsbetriebe, sind Modifikationen der Kompetenzprofile der Meisterebene sowie weniger hierarchische, stärker diskursive Kooperations- und Kommunikationspraktiken zwischen Tag- und Wechselschicht (Kap. 4).

Drittens schließlich ist auch Qualifikation, also der Facharbeiterabschluss, kein Garant für einen Anspruch auf Teilhabe an den Modernisierungsprozessen auf dem Shop floor. Die Chancen von den positiven Effekten der Aufwertung der Produktionsarbeit auch im Rahmen innovativer Gestaltungsansätze zu profitieren, sind unter den Produktionsbeschäftigten, dies zeigt unsere Empirie, ungleich verteilt. Noch allzu oft ist der Einsatz von Facharbeitern den Sicherheits- und Behördenanforderungen geschuldet, und wirken Markt-, Produkt- und Innovationsanforderungen eher hemmend als förderlich auf die neue Arbeitspolitik ein.

Eine vierte Differenzierung ist im Hinblick auf die Einbettung der neuen Arbeitsformen in die gesamtunternehmerischen Restrukturierungsprozesse vorzunehmen. Konnten Kern und Schumann in ihren Befunden zum „Ende der Arbeitsteilung?“ (1984) die Produktionsfacharbeiter in den industriellen Kernen der Neuen Produktionskonzepte noch als „Rationalisierungsgewinner“ bezeichnen, haben sich die Rahmenbedingungen für Arbeit in 
diesen Bereichen erheblich verschlechtert. Die im Vergleich zu anderen Industrien beachtlichen Entgelt- und Sozialstandards wurden seit Ende der 1990er Jahre zurückgefahren, und das Menetekel des Outsourcing oder der (feindlichen) Übernahme durch ein Konkurrenzunternehmen erhöhen auch in der Chemischen Industrie die Unsicherheiten. Tradierte Besitzstände werden immer brüchiger. Der Abbau von Sozialleistungen in der Chemischen Industrie und speziell die Abschaffung der „Treueprämie“ in einem Großunternehmen hat den Beschäftigten überdeutlich gemacht, dass sich unter den Bedingungen des „Turbo-Kapitalismus“ der Wert der Arbeitskraft beständig relativiert. Hinter dieser Entwicklung verbirgt sich indes nicht etwa eine dramatisch verschlechterte Performanz der Unternehmen. Im Gegenteil, die Umsatzrenditen sind, nach den Krisenjahren 1992/93 in der Regel überdurchschnittlich. Die Managementvertreter der Chemieunternehmen bekräftigen mit diesem Vorgehen den neuen Bezug auf Shareholder Value und Finanzmärkte. Eine „Treueprämie“ wird in diesem Umfeld schlicht und ergreifend als „nicht mehr zeitgemäß`“ definiert. ${ }^{185}$ Zusätzlich wird die kollektive Belegschaftsbindung über den Ausbau individueller Leistungsanteile von einer ergebnisorientierten Individualbeteiligung abgelöst (Kädtler 2003). Die traditionelle Chemiekultur, deren wesentliches Ziel die langfristige Bindung der Arbeitskräfte war, und die sich in einer umfassenden betrieblichen Sozialpolitik äußerte, erfährt damit eine harte Absage.

Längst geht es seitens der Belegschaften um mehr als Besitzstandswahrung. Die wie auch immer real begründete Angst, zur nächsten Outsourcing- oder Entlassungswelle zu zählen, wird durch die sich verschärfenden Bedingungen auf dem externen Arbeitsmarkt noch verstärkt. Denn das Segment der (Dauer-) Arbeitslosen ist bereits groß, die Exklusion Vieler struktureller Bestandteil der Gesellschaft (Vogel 2002). Für die meisten dieser Ausgeschlossenen sind die Hindernisse für einen Wiedereinstieg inzwischen unermesslich hoch - und sie steigen mit jedem Tag ohne Arbeit im erlernten Bereich. Eine Wiedereingliederung ist gerade in Bereichen mit aufgewerteter Produktionsarbeit und ihrem breitem Zugriff auf das Arbeitsvermögen voraussetzungsreich (Schumann 2003).

Bezogen auf die sozialen Strukturierungsprozesse in der Chemischen Industrie insgesamt wirken sich die skizzierten Entwicklungen in einem „Doppeleffekt betrieblichen Strukturwandels“ (Kurz 2002) aus, der bezogen auf die Gesamtbelegschaften in einem deutlich höheren Qualifikationsniveau zum Ausdruck kommt. Entwickelte sich die science based industry noch auf der Grundlage von Qualifikationsstrukturen, die durch einen hohen Anteil von Un- und Angelernten gekennzeichnet sind, nimmt nun die Bedeutung von technischen Angestellten, Naturwissenschaftlern und Ingenieuren zu. Besonders deutlich wird dies am Verhältnis von Arbeitern zu Angestellten, dass 1989 erstmals ausgeglichen ist und sich in den darauffolgenden Jahren weiter zuungunsten der Arbeiter verschiebt. Die interne Tertiarisierung schreitet in der Chemischen Industrie unaufhaltsam voran, und der Anteil an Organisations-, Führungs-, Forschungs- und Entwicklungsaufgaben wächst. ${ }^{186}$

\footnotetext{
${ }^{185}$ So äußerte sich der damalige Vorstandsvorsitzende Chem AG in einem Spiegel-Interview 1997.

${ }^{186}$ Vgl. zu interner Tertiarisierung und Frauenerwerbsbeschäftigung in der Automobil- und Elektroindustrie Kurz (2002)
} 
Parallel dazu sinkt innerhalb der Produktion der Anteil der Un- und Angelernten, wie die statistischen Erhebungen des BAVC aus dem Jahr 2001 belegen. Die „klassischen“ Entgeltstufen im Un- und Angelerntenbereich haben klar gegenüber den Facharbeiterpositionen verloren, und der in den 1980er Jahren einsetzende Trend zur Höherqualifizierung der Beschäftigten in der Produktion setzt sich fort.

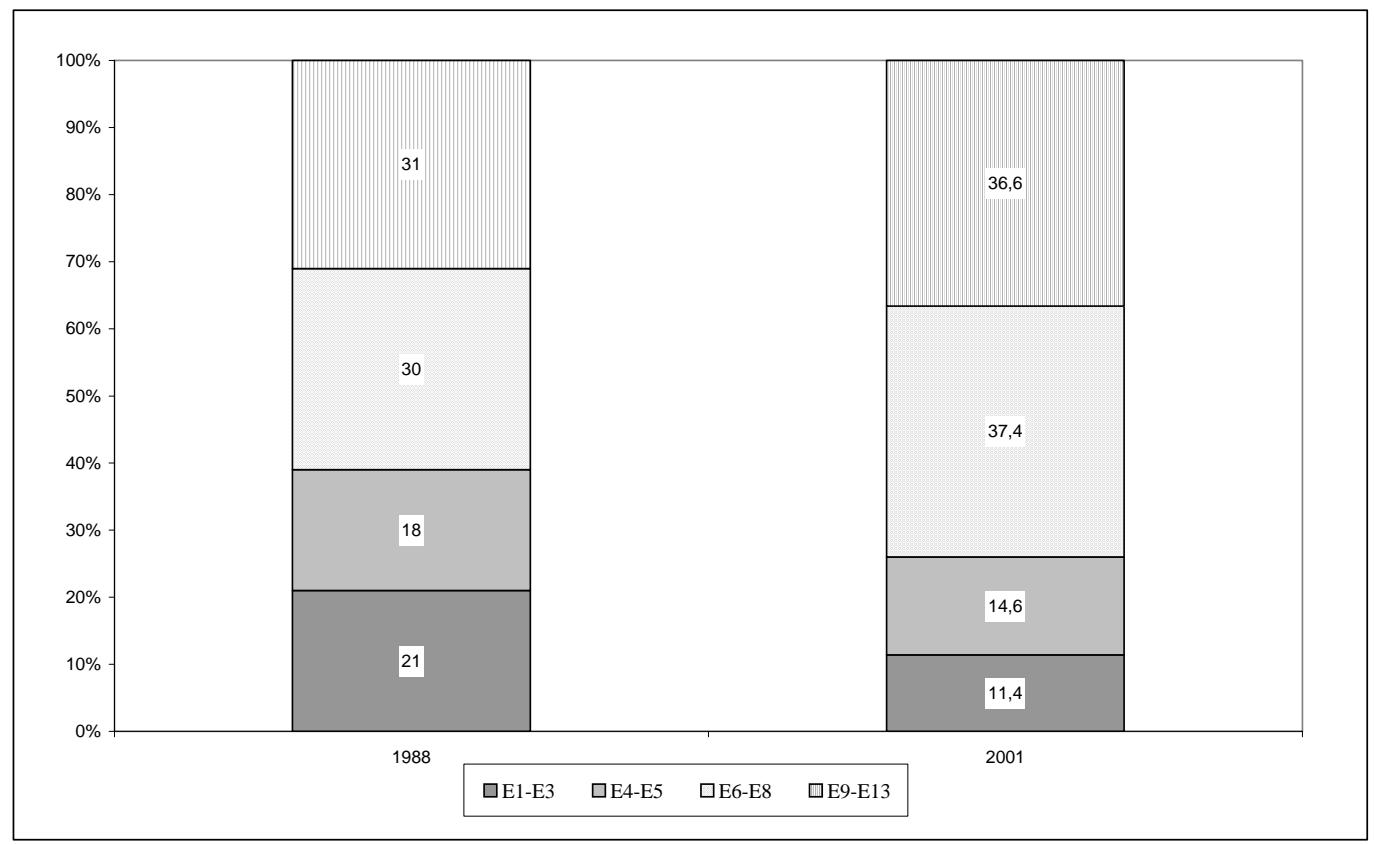

Abbildung 15: Verteilung der Entgeltgruppen in der Chemischen Industrie; BAVC (2002)

Eins zumindest steht wohl fest: Wer dem neuen Qualifikations- und Leistungsprofil nicht entspricht, der wird immer weiter auf die Verliererseite gedrängt. Exklusionseffekte lassen sich für nicht einschlägig qualifizierte, ältere Beschäftigte in von Auslagerung bedrohten Geschäftsfeldern und Arbeitsbereichen oder in den verbleibenden Bereichen der „einfachen“ Tätigkeiten, wie sie etwa an den Außenanlagen oder beim Abfüllen anfallen, erkennen. ${ }^{187}$ Zwar werden die Chemie-Sozialpartner nicht müde zu bekräftigen, dass Erhalt und Ausbau der Qualifikation der Belegschaften durch lebenslanges Lernen einer der

\footnotetext{
${ }^{187}$ Auf die Tatsache, dass bereits der einheitliche Entgeltrahmen, den die Chemie im Jahr 1987 abgeschlossen hat, eine Weichenstellung und gleichzeitige interne Marginalisierung der Un- und Angelernten bedeutet, kann hier nicht ausführlich eingegangen werden, zitiert sei zusammenfassend Kädtler (1987): „Während innerhalb der Bereiche von E1 bis E8 eine erhebliche Besserstellung der gelernten und ggf. weiterqualifizierten Facharbeiter und Handwerker stattfindet, der Bereich insgesamt aber durch die drastische Absenkung der Angestelltengruppen nach unten zusammengeschoben wird, wird die Absetzung der Angestelltengruppen E9 bis E13 von diesem Unterbau mittelfristiges Strukturmerkmal der Einkommenstarifpolitik in diesem Bereich. Schließlich Folge: eine klare, durch einen breiten Graben markierte Trennung eines tariflichen Unter- von einem tariflichen Obergeschoss. (...) Es liegt auf der Hand, dass es sich hier nicht nur um einen besonders gravierenden Tarifsprung handelt, sondern - allem Gerede von größerer Durchlässigkeit zum Trotz - um die eindeutige Scheidung einer unteren von einer oberen Beschäftigtenklasse. (...) An die Stelle der traditionellen Spaltung zwischen Arbeitern und Angestellten tritt damit eine sicherlich »modernere«, aber ebenso tiefe Spaltung zwischen Un-, Angelernten und Inhabern normaler Lehrabschlüsse, seien sie nun Arbeiter oder Angestellte, auf der einen Seite, und den Angestellten mit formal höherer Ausbildung bzw. Spezialqualifikationen auf der anderen Seite. Dass dabei die kleineren und die mittleren Angestellten die Höherstufung der höheren kaufmännischen Angestellten und Meister, die un- und angelernten Arbeiter und vor allem Arbeiterinnen die Aufbesserung der Facharbeiter und Handwerker (weniger: -innen) mitfinanzieren müssen, wirft ein nur zu bezeichnendes Licht auf die hier verwirklichte Vorstellung von Einkommensgerechtigkeit. Der Fortschritt fordert seine Opfer; und die liegen meistens unten.“
} 
wichtigsten Erfolgsfaktoren für den Standort Deutschland und für die Sicherung und Schaffung von Arbeitsplätzen sei. Dennoch hat der generelle Trend der Höherqualifizierung das Angebot an formeller betrieblicher Aus- und Weiterbildung für Un- und Angelernte bereits erheblich verringert, und Beschäftigungsunsicherheit und Gefahren der internen Ausgrenzung steigen erheblich.

Ob sich dieser Zugriff auf Arbeitskraft, dessen Leistungsanforderungen recht eindeutig auf jüngere und flexible Beschäftigte zielt, mittelfristig gegen die Unternehmen richtet, bleibt abzuwarten. Denn einmal ist nicht zu leugnen, dass erfahrungsgebundene Kompetenz in dieser Branche nach wie vor einen hohen Stellenwert hat, die Unterstützung der Beschäftigten also nicht leichtfertig verspielt werden sollte. Außerdem ist die Altersstruktur der Chemiebeschäftigten durch eine „demografischen Welle“ gekennzeichnet: Die starken Mittelbau- Jahrgänge der neunziger Jahre sind inzwischen in die nächsten Altersgruppen übergegangen, gleichzeitig bewirken die geburtenschwachen Jahrgänge eine relative Abnahme der jüngeren Teile der Belegschaften (BAVC 2003). Ein „pfleglicher“ Umgang mit menschlicher Arbeitskraft lohnt sich von daher mittel- bis langfristig auch aus unternehmerischer Perspektive.

Innovative Arbeitspolitik mit ihren Potentialen zur Verbesserung der Arbeitssituation und Belastungsregulation wäre dafür eine solide Basis - und nicht zuletzt mit den Veränderungen der betrieblichen Ausbildungssystematik gut vereinbar. Auf Initiative der Arbeitnehmer- wie Arbeitgeberorganisationen sind inzwischen einige Veränderungen im Bereich der beruflichen Ausbildung vorgenommen worden, die an die methodischen, sozialen und fachlichen Anforderungen der Prozessorganisation anknüpfen. ${ }^{188}$

- Seit August 2001 gilt für Chemikanten eine „modernisierte“ Ausbildungsordnung. Im Einleitungstext wird explizit auf neue Formen der Arbeitsgestaltung bezug genommen. ${ }^{189}$ Zum „Leitbild“ der Erstausbildung werden Beschäftigte, die in Teamarbeit Prozessabläufe steuern, Maßnahmen zur schnellen Fehler- und Störbehebung treffen und für Wartung und Instandhaltung sorgen. Die Auszubildenden sollen deshalb in der Ausbildung befähigt werden, entlang einer Prozesskette zu arbeiten und alle dabei anfallenden Arbeiten - auch fachfremder Art - zu erledigen (BiBB 2001).

\footnotetext{
${ }^{188}$ Schon Mitte der 1990er Jahre wurden in einer Reihe von Chemieunternehmen Modellversuche gestartet, die auf Defizite in der fachübergreifenden Zusammenarbeit der Auszubildenden im Bereich Metall, Elektro und Chemie zielten, so etwa „BETA“, berufsübergreifende, teamorientierte Ausbildung, bei Hoechst, „Industrielandschaften“ bei BASF, die „Teamausbildung“ bei Bayer sowie ein vom BiBB gefördertes Projekt. In allen Fällen handelt es sich um Versuche, die Teamfähigkeit von Chemikanten, Pharmakanten, Anlagenmechaniker, Industriemechaniker, Energieelektroniker und Prozessleitelektroniker zur gemeinsamen Bewältigung von Produktionsprozessen zu erhöhen. Im Abschlussbericht des BiBB-Projekts heißt es in einer Zusammenfassung: „Vorgestellt wird ein Modellversuch zur interdisziplinären und fallbezogenen Ausbildung von zukünftigen Anlagenfahrern und Instandhaltern in der Chemie- und Papierindustrie. Nach drei Ausbildungsjahren sind Wirkungen des Modellversuchs auf das emotionalen Erleben der Auszubildenden (Vertrauen in Ausbildungskollegen und Ausbilder, Zusammenhalt in der Ausbildungsgruppe), die Intentionen der Auszubildenden (Absicht, mit Kollegen anderer Berufsgruppen zusammenzuarbeiten u.a.) und die Ausbildungsleistungen (Erfüllung der Aufgaben und Pflichten, Unterstützung von Kollegen, Fehlzeiten der Auszubildenden) nachweisbar. Aufgrund der positiven Wirkungen des Modellversuchs wurde das hier vorgestellte neue Ausbildungskonzept in dem Chemieunternehmen im Herbst 1997 zur Regelausbildung übernommen.“ (Kubik et al. 1999 :1)

189 „Neue Arbeitsformen wie z.B. Gruppenarbeit und prozessorientierte interdisziplinäre Zusammenarbeit prägen in zunehmendem Maße die Facharbeit von Chemikanten / Chemikantinnen. Dabei spielen - neben fachlichen (chemisch-technischen) Qualifikationen insbesondere berufsübergreifende Qualifikationen eine wesentliche Rolle“. (BiBB 2001: 33)
} 
- Auf Initiative der Sozialpartner ist ebenfalls eine Neuordnung des Ausbildungsgangs „Industriemeister mit Fachrichtung Chemie“ auf den Weg gebracht. Die notwendigen Eckwerte des Verfahrens sind beschrieben und im Jahr 2003 abgeschlossen worden. Arbeitnehmer wie Arbeitgeberorganisationen bekräftigen darin die Rolle des Meisters als „Förderer, Entwickler und Unterstützer seiner Mitarbeiter, also seine Rolle als „Coach“. In der Ausbildung sollen von daher zukünftig die entsprechenden Kompetenzen und die Vorbereitung auf die damit einher gehende Verantwortung gefördert werden (IG BCE 2003).

Diese parallele Stärkung prozessbezogener Kompetenzen und berufsfachlich ausgerichteter Qualifikationen, wie sie in den neuen Ausbildungsordnungen umgesetzt ist, scheint eine einigermaßen adäquate Vorbereitung auf die Arbeit in der prozessorientierten Arbeits- und Betriebsorganisation zu sein. Die Ergebnisse dieser Arbeit deuten aber an, dass die Kohärenz von bildungs- und arbeitspolitischen Zielsetzungen in der Praxis noch eher die Ausnahme denn die Regel ist. 


\section{Literaturverzeichnis}

Abelshauser, W., (Hrsg), 2002: Die BASF: Eine Unternehmensgeschichte. München: Beck.

Aglietta, M., 2000: Ein neues Akkumulationsregime: die Regulationstheorie auf dem Prüfstand. Hamburg: VSA.

Altvater, E./Fehrmann, E., (Hrsg.), 1999: Turbo-Kapitalismus: Gesellschaft im Übergang ins 21. Jahrhundert. Hamburg: VSA.

Badham, R./Jürgens, U., 1998: Images of Good Work and the Politics of Teamwork, in: Economic and Industrial Democracy, 19 (1), S. 33-58.

Baecker, D., 1999: Organisation als System. Frankfurt: Suhrkamp.

Baethge, M./Baethge-Kinsky, V., 1998: Der implizite Innovationsmodus: Zum Zusammenhang von betrieblicher Arbeitsorganisation, human resources development und Innovation. in: F. Lehner (Hrsg.): Beschäftigung durch Innovation. Eine Literaturstudie. München und Mering: Rainer Hampp Verlag.

Baethge, M./Denkinger, J./Kadritzke, U., 1995: Das Führungskräfte-Dilemma. Frankfurt/New York: Campus.

Baethge-Kinsky, V. H., Thomas, 2001: Kompetenzentwicklung als Transformation der betrieblichen Sozialorganisation., S. 19-100 in: A. Qualifikations-EntwicklungsManagement. (Hrsg.): Kompetenzen entwickeln - Veränderungen gestalten. Münster; New York; München; Berlin: Waxmann.

Bathelt, H., 1997: Chemiestandort Deutschland. Technologischer Wandel, Arbeitsteilung und geographische Struktur in der Chemischen Industrie. Berlin: Edition Sigma.

Bathelt, H., 1997b: Im Strukturwandel gut behauptet. Strukturwandel in der deutschen Chemie: Teil 3., in: Chemie-Produktion, 1/97, S. 18-20.

Baumgarten, M., 1997: Professoren und Universitäten im 19. Jahrhundert: zur Sozialgeschichte deutscher Geistes- und Naturwissenschaftler. Göttingen: Vandenhoeck \& Ruprecht.

Bäumler, E., 1963: Ein Jahrhundert Chemie. Düsseldorf: Econ.

Bayer AG: Geschäftsbericht. diverse Jahrgänge.

Beck, U., 1986: Risikogesellschaft. Auf dem Weg in eine andere Moderne. Frankfurt: Suhrkamp.

Becker, S., 2001: Einfluss und Grenzen des Shareholder Value. Strategie- und Strukturwandel deutscher Großunternehmen der chemischen und pharmazeutischen Industrie. Frankfurt/Berlin/Brüssel/New York/Oxford/Wien: Lang.

Behr, M., 1995: Regressive Gemeinschaft oder zivile Vergemeinschaftung? Ein Konzept zum Verständnis posttraditionaler Formen betrieblicher Sozialintegration., in: Zeitschrift für Soziologie, 24 Heft 5, S. 325-344.

Behrens, M./Hardwig, T./Kädtler, J., 1996: Der Industriemeister und der Strukturwandel in der Industrie - Neue Variationen zu einem alten Thema., in: SOFI-Mitteilungen, 23, S. 25-44.

Bender, G., (Hrsg), 2001: Neue Formen der Wissenserzeugung. Frankfurt/New York: Campus.

Benders, J./Huijgen, F./Pekruhl, U., 2000: Gruppenarbeit in Europa - Ein Überblick, in: WSI-Mitteilungen, 6: 365-374. 
Benders, J./Van Hootegem, G. (1996): A team is not a team; towards a typology for comparing shopfloor teams. S.1-20.

Benders, J./Van Hootegem, G., 1999: Teams and Their Context. Moving the team discussion beyond dichotomies., in: Journal of Management Studies, 36 (5).

BiBB 2001: Neue und modernisierte Ausbildungsberufe 2001. Bonn.

Blauner, R., 1964: Alienation and Freedom: the factory worker and his industry. Chicago: University of Chicago Press.

Böhle, F./Bolte, A./Drexel, I./Dunkel, W./Pfeiffer, S./Porschen, S., 2002: Umbrüche im gesellschaftlichen Umgang mit Erfahrungswissen. Theoretische Konzepte, empirische Befunde, Perspektiven der Forschung. München: ISF.

Böhle, F./Rose, H., 1992: Technik und Erfahrung - Arbeit in hochautomatisierten Systemen. Frankfurt, New York: Campus.

Bönig, J., 1993: Die Einführung von Fließbandarbeit in Deutschland bis 1933 : zur Geschichte einer Sozialinnovation. Münster.

Borkin, J., 1978: Die unheilige Allianz der I.G. Farben. Eine Interessengemeinschaft im Dritten Reich. Frankfurt, New York: Campus.

Boyer, R., 1992: Neue Richtungen von Managementpraktiken und Arbeitsorganisation. Allgemeine Entwicklungen und nationale Entwicklungspfade., S. 55-103 in: A. Demirovic/H.-P. Krebs/T. Sablowski (Hrsg.): Hegemonie und Staat. Kapitalistische Regulation als Projekt und Prozess. Münster: Westfälisches Dampfboot.

Braczyk, H.-J./Schienstock, G., 1996: Im 'Lean-Express' zu einem neuen Produktionsmodell? 'Lean Production ' in Wirtschaftsunternehmen Baden-Württembergs - Konzepte, Wirkungen, Folgen. in: H.-J. Braczyk/G. Schienstock (Hrsg.): Kurswechsel in der Industrie. Lean Production in Baden-Württemberg.

Brandt, G., 1987: Fragen der betrieblichen Arbeitsgestaltung im Kontext der technischwissenschaftlichen Entwicklung. in: D. Bieber/W. Schumm (Hrsg.): Arbeit, Technik und gesellschaftliche Entwicklung. Transformationsprozesse des modernen Kapitalismus. Aufsätze 1971-1987. Frankfurt a.M.

Braverman, H., 1977: Die Arbeit im modernen Produktionsprozess. Frankfurt/New York: Campus.

Briken, K./Kurz, C., 2003: "Wir können nicht mehr alles Mögliche unter dem Deckmantel Basic Research machen..." Neue Formen der Nutzung und Steuerung wissenschaftlicher Arbeits(kraft) in der Pharma- und Biotechindustrie., in: SOFIMitteilungen, 31.

Briken, K, 1999: Nicht nur die Chemie muss stimmen. Eine Untersuchung zur betrieblichen Restrukturierung in der Chemischen Industrie., in: SOFI Mitteilungen, 27, S.8396

Bruns, M./Thietke, J., 1997: Forschung und Entwicklung - Beschäftigungs- und Standortsicherung in der Chemischen Industrie. Düsseldorf.

Bungard, W./Jöns, I., 1997: Gruppenarbeit in Deutschland - Eine Zwischenbilanz, in: Zeitschrift für Arbeits- und Organisationspsychologie, 41, S. 104-119.

Burchardt, L., 1979: Die Zusammenarbeit zwischen chemischer Industrie, Hochschulchemie und chemischen Verbänden im Wilhelminischen Deutschland, in: Technikgeschichte, 46, S. 194-200. 
Burchardt, L., 1992: Wissenschaft, Industrie und Kultur zur Zeit August Wilhelm Hofmanns, S. 7-26 in: C. Meinel/H. Scholz (Hrsg.): Die Allianz von Wissenschaft und Industrie August Wilhelm Hofmann (1818-1892). Weinheim, New York, Basel: $\mathrm{VCH}$.

Burke, P., 2001: Papier und Marktgeschrei. Frankfurt: Wagenbach.

Castells, M., 2003: Der Aufstieg der Netzwerkgesellschaft. Opladen: Leske+Budrich.

Cattero, B., 1998: Mythos Facharbeit. Anmerkungen aus ausländischer Sicht über die 'Verberuflichung' des industriesoziologischen Diskurses in Deutschland, in: SOFIMitteilungen, 26, S. 99-116.

Chandler, A. D., 1990: Scale and Scope: The Dynamikcs of Industrial Capitalism. Cambridge/MA.: Harvard University Press.

Christ, M., 1995: Anforderung - Eignung - Ausbildung. Die Rolle unternehmerischer Anstrengungen zur Mitarbeiterqualifikation im Industrialisierungsprozeß am Beispiel der Maschinenbau- , der Elektro- und der Chemischen Industrie in der zweiten Hälfte des 19. Jahrhunderts und am Anfang des 20. Jahrhunderts. Mainz.

Crozier, M./Friedberg, E., 1993: Die Zwänge kollektiven Handelns. Über Macht und Organisation. Frankfurt: Athenäum.

D'Alessio, N./Oberbeck, H./Seitz, D., 2000: Rationalisierung in Eigenregie. Ansatzpunkte für den Bruch mit dem Taylorismus bei VW. Hamburg: VSA-Verlag.

Deutschmann, C., 1989: Reflexive Verwissenschaftlichung und kultureller 'Imperialismus' des Managements., in: Soziale Welt, 3, S. 374-396.

Deutschmann, C., 1995: Dezentralisierung: Unternehmen zwischen Stabilität und Desintegration, in: Zeitschrift für Soziologie, 24 Heft 6, S. 422-435.

Deutschmann, C., 1997: Die Mythenspirale. Eine wissenssoziologische Interpretation industrieller Rationalisierung., in: Soziale Welt, 47, S. 55-70.

Deutschmann, C., 1999: Die Verheißung des absoluten Reichtums. Zur religiösen Natur des Kapitalismus. Frankfurt a.M., New York: Campus-Verlag.

Dolata, U., 1997: Die Realität der Globalisierung: Bayer, Hoechst, BASF, in: Blätter für deutsche und internationale Politik, 42 (1997), S. 1380-1383.

Dolata, U., 2002: Unternehmen Technik. Akteure, Interaktionsmuster und strukturelle Kontexte der Technikentwicklung: Ein Theorierahmen. Berlin: Edition Sigma.

Dörre, K., 1996: Die "demokratische Frage" im Betrieb - Zu den Auswirkungen partizipativer Managementkonzepte auf die Arbeitsbeziehungen in deutschen Industrieunternehmen., in: SOFI-Mitteilungen, 23, S. 7-24.

Dörre, K./Neubert, J./Wolf, H., 1993: "New Deal" im Betrieb? Unternehmerische Beteiligungskonzepte und ihre Wirkung auf die Austauschbeziehungen zwischen Management, Belegschaften und Interessenvertretungen., in: SOFI-Mitteilungen, 20, S. 1536.

Drews, J., 1998: Die verspielte Zukunft. Wohin geht die Arzneimittelforschung? Basel/Boston/Berlin: Birkhäuser Verlag.

Drexel, I., 1982: Belegschaftsstrukturen zwischen Veränderungsdruck und Beharrung. Zur Durchsetzung neuer Ausbildungsberufe gegen bestehende Qualifikations- und Lohnstrukturen. Frankfurt: Campus-Verlag. 
Drexel, I./Nuber, C., 1979: Qualifizierung für Industriearbeit im Umbruch. Die Ablösung von Anlernung durch Ausbildung in großbetrieben von Stahl und Chemie. Frankfurt: Campus-Verlag.

Edwards, P./Wright, M., 1998: Does Teamwork Work and if so, Why? A Case Study in the Aluminium Industry, in: Economic and Industrial Democracy, 19 (1), S. 59-90.

Etzkowitz, H./Webster, A., 1998: Entrepreneurial Science: The Second Academic Revolution, S. 21-46 in: H. Etzkowitz/A. Webster/P. Healey (Hrsg.): Capitalizing Knowledge. New Intersections of Industry and Academia. New York.

Faust, M./Jauch, P./Brünnecke, K./Deutschmann, C., 1994: Dezentralisierung von Unternehmen. Bürokratie- und Hierarchieabbau und die Rolle betrieblicher Arbeitspolitik. München und Mering: Rainer Hampp Verlag.

Festel, G./ Knöll, J./ Götz, H./ Zinke, H., 2004: Der Einfluss der Biotechnologie auf Produktionsverfahren in der Chemieindustrie. In: Chemie, Ingenieur, Techniker, 76, Nr. 3, S. 307-312

Frieling, E./Freiboth, M., 1997: Klassifikation von Gruppenarbeit und Auswirkungen auf subjektive und objektive Merkmale der Arbeitstätigkeit, in: Zeitschrift für Arbeitsund Organisationspsychologie, 41, S. 120-130.

Fröhlich, D., 1983: Machtprobleme in teilautonomen Arbeitsgruppen, in: KZfSS, Sonderheft 25, S. 532-551.

Fürstenberg, F., 1969: Die Soziallage der Chemiearbeiter. Industriesoziologische Untersuchungen in rationalisierten und automatisierten Chemiebetrieben. Neuwied, Berlin: Luchterhand.

Gerst, D., 2000: Arbeitspolitik im Rückwärtsgang? Konzeptionskonkurrenz und Wandel von Kontrolle in der Automobilindustrie., in: WSI-Mitteilungen, 1/2000, S. 37-45.

Gerst, D./Hardwig, T./Kuhlmann, M./Schumann, M., 1995: Gruppenarbeit in den 90ern: Zwischen strukturkonservativer und strukturinnovativer Gestaltungsvariante., in: SOFI-Mitteilungen, 22, S. 39-65.

Gerst, D./Schumann, M., 1997: Produktionsarbeit - Bleiben die Entwicklungstrends stabil? in: (Hrsg.): Jahrbuch sozialwissenschaftliche Berichterstattung. Berlin: Sigma.

Giddens, A., 1997: Die Konstitution der Gesellschaft: Grundzüge einer Theorie der Strukturierung. Frankfurt / Main; New York: Campus-Verlag.

Glißmann, W. (1996): Die neue Organisation der Arbeit. Arbeitsnehmer als "unselbständige Selbständige" im Unternehmen \& neue Voraussetzungen für kollektives Handeln. S. 1-22.

Göranzon, B./Josefson, I., (Hrsg.), 1988: Knowledge, Skill and Artificial Intelligence. Berlin, Heidelberg, New York.

Greb, R., 2000: Zentralisierung in der globalen Unternehmung: die Organisation unternehmensinterner FuE in der chemischen Industrie. Wiesbaden: Deutscher Universitäts Verlag.

Haber, L. F., 1982: Chemical Innovation in Peace and War, S. 271-282 in: C. G. Bernhard/E. Crawford/P. Sörbom (Hrsg.): Science, Technology and Society in the Time of Alfred Nobel. Oxford, New York: Pergamon Press.

Hack, L., 1998: Technologietransfer und Wissenstransformation. Zur Globalisierung der Forschungsorganisation von Siemens. Münster: Westfälisches Dampfboot. 
Hack, L., 2001: "Ich habe da eine Theorie" oder: Neue Fokussierung vom Kontext/en und Kompetenz/en, S. 23-52 in: G. Bender (Hrsg.): Neue Formen der Wissenserzeugung. Frankfurt/New York: Campus.

Hack, L., 2002: Organisationsvermögen. Gesellschaftliche Formbestimmungen von Wissen in globalisierten Kontexten., in: Das Argument (248), S. 668-683.

Hack, L./Hack, I., 1985: Die Wirklichkeit, die Wissen schafft. Zum wechselseitigen Begründungsverhältnis von 'Verwissenschaftlichung der Industrie' und 'Industrialisierung der Wissenschaft'. Frankfurt: Campus.

Hammer, M./Champy, J., 1994: Business Reengineering. Die Radikalkur für das Unternehmen. Frankfurt/New York: Campus.

Heidenreich, M., 1998: Die duale Berufsausbildung zwischen industrieller Prägung und wissensgesellschaftlichen Herausforderungen., in: Zeitschrift für Soziologie., 27 (1998) (5), S. 321-340.

Heidenreich, M., 1998b: Die Organisation von Arbeit in der Wissensgesellschaft, in: Industrielle Beziehungen, 5: 13-44.

Heidenreich, M. (2002): Konturen der Wissensgesellschaft. Vortrag an der Universität Zürich.

Heine, H./Mautz, R., 1989: Industriearbeiter contra Umweltschutz? Frankfurt a.M., New York: Campus.

Helfert, M./Trautwein-Kalms, G., 2000: Arbeitspolitik unter Bedingungen der Flexibilisierung und "Globalisierung". Vom Gestaltungsanspruch zur arbeitspolitischen Abstinenz - Neue Perspektiven?, in: WSI-Mitteilungen, 1/2000, S. 1-4.

Hertle, H. H./Kädtler, J., 1990: Die industriepolitische Wende der industriellen Beziehungen - Gewerkschaftspolitik unter dem Primat der Industriepolitik am Beispiel der IG Chemie/Papier/Keramik, in: Soziale Welt, 2, S. 183-205.

Hesse, H. A., 1968: Berufe im Wandel. Ein Beitrag zum Problem der Professionalisierung. Stuttgart: Ferdinand Enke.

Hirsch, J./Roth, R., 1986: Das neue Gesicht des Kapitalismus: vom Fordismus zum PostFordismus. Hamburg: VSA-Verlag.

Hirschhorn, L., 1984: Beyond mechanization : work and technology in a postindustrial age. Cambridge, Ma.: MIT-Press.

Hirschman, A. O., 1974: Abwanderung und Widerspruch: Reaktionen auf Leistungsabfall bei Unternehmungen, Organisationen und Staaten. Tübingen: Mohr.

Hofmann, H./Röben, P., 1997: Berufsübergreifende Ausbildung im Team - aktuelle Entwicklungen in der Chemischen Industrie, in: Lernen \& Lehren, 12 (47), S. 60-74.

Hofmann, R., 1975: Welt-Chemiewirtschaft. Entwicklungstendenzen. Opladen: Westdeutscher Verlag.

Jauch, P., 1997: Industriemeister und industrielle Reorganisation. München und Mering: Rainer Hampp Verlag.

Jauch, P./Faust, M., 1998: Die Zukunft des Industriemeisters in der dezentralisierten Organisation., S. 199-228 in: R. Jansen/O. Hecker/D. Scholz (Hrsg.): Facharbeiteraufstieg in der Sackgasse? Entwicklungen und Perspektiven auf der mittleren Qualifikationsebene. Bielefeld: W. Bertelsmann.

Jürgens, U., 1997: Rolling Back Cycle Times: The Renaissance of the Classic Assembly Line in Final Assembly, S. 255-273 in: K. Shimokawa/U. Jürgens/T. Fujimoto 
(Hrsg.): Transforming Automobile Assembly. Experience in Automation and Work Organization. Berlin, Heidelberg, New York: Springer Verlag.

Jürgens, U./Lippert, I., 1998: Schnittstellen des deutschen Produktionsregimes. Innovationshemmnisse im Produktentstehungsprozeß. S. 65-94 in: F. Naschold/D. Soskice/B. Hancké/U. Jürgens (Hrsg.): Ökonomische Leistungsfähigkeit und institutionelle Innovation. Das deutsche Produktions- und Politikregime im globalen Wettbewerb. Berlin: Edition Sigma.

Jürgens, U./Naschold, F., 1994: Arbeits- und industriepolitische Entwicklungsengpässe der deutschen Industrie in den neunziger Jahren., S. 239-270 in: W. Zapf/M. Dierkes (Hrsg.): Institutionenvergleich und Institutionendynamik. Berlin: Edition Sigma.

Kädtler, J., 1998: Globalisierung aus Arbeitnehmerinteressen - oder: wie aus einfachen Antworten komplizierte Fragen entstehen., in: SOFI-Mitteilungen, 26, S. 69-80.

Kädtler, J., 2000: Die Großen werfen ihre Netze aus - Zum Verhältnis von Zentralisierung und Netzwerkkonfigurationen in der deutschen Chemieindustrie. in: H. Minssen (Hrsg.): Begrenzte Entgrenzungen. Berlin: Edition Sigma.

Kädtler, J., 2003: Globalisierung und Industrielle Beziehungen in Deutschland - am Beispiel der Chemischen Industrie. unveröffentlichtes Manuskript.

Kädtler, J./Hardwig, T., 1998: Motor oder Verschleißteil im modernisierten Industriebetrieb - Perspektiven für die Industriemeisterposition. in: R. Jansen/O. Hecker/D. Scholz (Hrsg.): Facharbeiteraufstieg in der Sackgasse? Entwicklungen und Perspektiven auf der mittleren Qualifikationsebene. Bielefeld: W. Bertelsmann.

Kädtler, J./Hertle, H. H., 1997: Sozialpartnerschaft und Industriepolitik : Strukturwandel im Organisationsbereich der IG Chemie-Papier-Keramik. Opladen: Westdeutscher Verlag.

Kern, H./Bernoux, P., 1997: Bottom-up oder top-down? Ein deutsch-französischer Dialog über Vertrauen als Innovationsfaktor. Opladen: Leske \& Budrich.

Kern, H./Sabel, C. F., 1994: Verblaßte Tugenden. Zur Krise des deutschen Produktionsmodells., S. 605-624 in: Umbrüche gesellschaftlicher Arbeit. (Soziale Welt Sonderband). Göttingen: Otto Schwartz \& Co.

Kern, H./Schumann, M., 1985: Industriearbeit und Arbeiterbewußtsein. Eine empirische Untersuchung über den Einfluß der aktuellen technischen Entwicklungen auf die industrielle Arbeit und das Arbeiterbewußtsein. Mit einem Nachwort von Klaus Peter Wittemann. Frankfurt: Suhrkamp.

Kern, H./Schumann, M., 1990: Das Ende der Arbeitsteilung? Rationalisierung in der industriellen Produktion. 4. Aufl. München: Verlag C.H. Beck.

Kießling, B., 1988: Kritik der Giddensschen Sozialtheorie. Frankfurt am Main; Bern; New York; Paris: Peter Lang.

Klapper, N., 1995: Produktion und Technik rücken organisatorisch näher zusammen., in: Harvard Business Manager, 2 (1995): 69-79.

Knorr-Cetina, K., 2002: Wissenskulturen. Ein Vergleich naturwissenschaftlicher Wissensformen. Frankfurt: Suhrkamp.

Kocyba, H., 2000: Jenseits von Taylor und Schumpeter: Innovation und Arbeit in der "Wissensgesellschaft", S. 25-58 in: ISF München (Hrsg.): Innovation und Arbeit. Berlin: Sigma. 
König, W., 1995: Technikwissenschaften. Die Entstehung der Elektrotechnik aus Industrie und Wissenschaft zwischen 1880 und 1914. Chur: G+B Verlag Fakultas.

Kotthoff, H./Reindl, J., 1990: Die soziale Welt kleiner Betriebe. Wirtschaften, Arbeiten und Leben im mittelständischen Industriebetrieb. Göttingen.

Koubek, N./Cleff, T./Pierotti, C./Schafmeister, S., 1996: Unternehmensstrategien in der Triade. Baden-Baden: Nomos Verlagsgesellschaft.

Kripzak, P., 1994: Akzeptanz verbessern - Industriepolitik betrieben - die Zukunft der chemischen Industrie sichern., S. 216-224 in: R. Simons/K. Westermann (Hrsg.): Wirtschaftsstandort Deutschland. Wettbewerbsfähigkeit. Zukunftschancen. Verteilungsspielräume. Köln: Bund-Verlag.

Kubik, G./Grote, K./Kappe, A./Schmidkunz, H./Schröder, L./Waasdorp, A./Werthebach, M., 1999: Modellversuch zur Verbesserung der Teamfähigkeit von Chemikanten / Papiermachern, Industriemechanikern und Prozessleitelektronikern / Energieelektronikern für die gemeinsame Bewältigung von Produktionsprozessen.

Kühl, S., 1995: Wenn die Affen den Zoo regieren. Frankfurt a.M., New York: Campus Verlag.

Kuhlmann, M./ Balzert, S./Sperling, H.-J., 2004: Konzepte innovativer Arbeitspolitik. Good practice aus vier Branchen. Edition Sigma. Berlin.

Kuhlmann, M./Gerst, D. (1997): Thesen zum Arbeitsverständnis bei selbstorganisierter Gruppenarbeit: 1-17. unveröffentlichtes Manuskript.

Kuhlmann, M./Kurz, C., 1995: Strukturwandel der Arbeit? Betriebliche Reorganisation und die Bedeutung sozialer Strukturen, in: SOFI-Mitteilungen, 22, S. 31-38.

Kuhlmann, M./Schumann, M., 2000: Was bleibt von der Arbeitersolidarität? Zum Arbeits- und Betriebsverständnis bei innovativer Arbeitspolitik, in: WSI-Mitteilungen, 1/2000: 18-27.

Kühnlein, G., 1990: Ausbildung und Arbeitserfahrung eines 'Zukunftsberufs' im Widerstreit - Am Beispiel der Chemikantenausbildung in der Großchemie. in: H. Friebel (Hrsg.): Berufsstart und Familiengründung - Ende der Jugend? Opladen: Westdeutscher Verlag.

Kurz, C., 1999: Repetitivarbeit - unbewältigt. Betriebliche und gesellschaftliche Entwicklungsperspektiven eines beharrlichen Arbeitstyps. Berlin: Edition Sigma.

Kurz, C., 2000: "Nicht nur Techniker sein" - Zur Beschäftigung und Arbeit von Ingenieuren in der Industrie, S. 59-106 in: ISF München (Hrsg.): Innovation und Arbeit. Berlin: Sigma.

Kurz, C., 2002: Innovation und Kompetenzen im Wandel industrieller Organisationsstrukturen, in: Mitteilungen aus der Arbeitsmarkt- und Berufsforschung, 35, S. 601615.

Kurz, C., 2002b: Frauenbeschäftigung und Strukturierungsprozesse der Erwerbsarbeit in der Automobil- und Elektroindustrie, in: SOFI-Mitteilungen, 30, S. 161-189.

Kurz, C./Mickler, O., 2001: Neue Anforderungsprofile und Perspektiven der Kompetenzentwicklung für Ingenieure in: B. Lutz/P. Meil/B. Wiener (Hrsg.): Industrielle Fachkräfte für das 21. Jahrhundert. Aufgaben und Perspektiven für die produktion von morgen. München: Campus.

Landes, D. S., 1973: Der entfesselte Prometheus. Köln. 
Landes, D. S., 1999: Wohlstand und Armut der Nationen. Warum die einen reich und die anderen arm sind. Berlin: Siedler.

Leimbach, A.,1999: Kein Spaß an der Arbeit im Team. VDI Nachrichten Nr. 15.

Luhmann, N., 1990: Die Wissenschaft der Gesellschaft. Frankfurt: Suhrkamp.

Luhmann, N., 2000: Organisation und Entscheidung. Opladen: Westdeutscher Verlag.

Lundgreen, P./Grelon, A., 1994: Ingenieure in Deutschland, 1770-1990. Frankfurt, New York: Campus Verlag.

Lutz, B., 1984: Der kurze Traum immerwährender Prosperität: eine Neuinterpretation der industriell-kapitalistischen Entwicklung im Europa des 20. Jahrhunderts. Frankfurt: Campus.

Lyotard, J.-F., 1999: Das postmoderne Wissen. Wien: Passagen.

Mach, E., 1975: Entwerfen und Bauen. Von der Zusammenarbeit der Architekten und Ingenieure mit Chemikern und Physikern in einem chemischen Großbetrieb. Ludwigshafen: Kastner \& Callwey.

Malsch, T., 1987: 'Neue Produktionskonzepte' zwischen Rationalität und Rationalisierung - Mit Kern und Schumann auf Paradigmensuche., S. 53-80 in: T. Malsch/R. Seltz (Hrsg.): Die neuen Produktionskonzepte auf dem Prüfstand. Beiträge zur Entwicklung der Industriearbeit. Berlin: Edition Sigma.

March, J. G./Simon, H. A., 1958: Organizations. New York.

Mason, G./Wagner, K., 1994: Innovation and the Skill Mix: Chemicals and Engineering in Britain and Germany, in: National Institue Economic Review, 148 (May), S. 61-72.

McClelland, C. E., 1980: State, society, and university in Germany 1700-1914. Cambridge, New York: Cambridge University Press.

McClelland, C. E., 1991: The German experience of professionalization. Modern learned professions and their organizations from the early nineteenth century to the Hitler era. Cambridge, New York: Cambridge University Press.

Menz, W./Becker, S./Sablowski, T., 1999: Shareholder-Value gegen Belegschaftsinteressen. Der Weg der Hoechst-AG zum "Life-Sciences"-Konzern. Hamburg: VSAVerlag.

Merton, R. K., 1985: Entwicklung und Wandel von Forschungsinteressen. Aufsätze zur Wissenschaftssoziologie. Frankfurt: Suhrkamp.

Mickler, O./Dittrich, E./Neumann, U., 1975: Technik, Arbeitsorganisation und Arbeit. Eine empirische Untersuchung in der automatisierten Produktion. Göttingen: SOFI.

Minssen, H., 1999: Von der Hierarchie zum Diskurs? Die Zumutungen der Selbstregulation. München und Mering: Rainer Hampp Verlag.

Moldaschl, M., 1994: "Die werden zur Hyäne" - Erfahrungen und Belastungen in neuen Arbeitsformen, S. 105-149 in: M. Moldaschl/R. Schultz-Wild (Hrsg.): Arbeitsorientierte Rationalisierung: Fertigungsinseln und Gruppenarbeit im Maschinenbau. Frankfurt, New York: Campus.

Mühlbauer, F., 1961: Naturwissenschaftlich orientierte Berufe, in: Aus der Praxis betrieblicher Sozialpolitik, 8 (Grundlagen der Berufsausbildung), S. 8-18.

Negroponte, N., 1995: Being Digital. London: Knopf.

Noble, D. F., 1977: America By Design. Science, Technology, and the Rise of Corporate Capitalism. Oxford: University Press. 
Ortmann, G., 1995: Formen der Produktion. Organisation und Rekursivität. Opladen: Westdeutscher Verlag.

Ortmann, G./ Sydow, J./ Windeler, A. (1997): Organisation als reflexive Strukturation. in: Ortman, G.; Sydow, J,.; Türk, K.: Theorien der Organisation. Opladen. Westdeutscher Verlag. S. 315-354.

Ötinger, B. v., 1994: Das Boston-Consulting-Group-Strategie-Buch: die wichtigsten Managementkonzepte für den Praktiker. Düsseldorf: Econ.

Paul-Kohlhoff, A., 1989: Wandel des Arbeitskräfteeinsatzes in der Chemieindustrie - Ergänzende Bemerkungen zur Expertise von H. Wöcherl, in: L. Pries/R. Trinczek/R. Schmidt (Hrsg.): Trends betrieblicher Produktionsmodernisierung. Opladen: Westdeutscher Verlag. S. 261-265

Peetz, H., 1981: "Nicht ohne uns!" Arbeiterbriefe, Berichte und Dokumente zur chemischen Industrialisierung von 1760 bis heute. Frankfurt: Ullstein.

Picot, A./Reichwald, R./Wiegand, R. T., 1998: Die grenzenlose Unternehmung. Information, Organisation und Management. Lehrbuch zur Unternehmensführung im Informationszeitalter. Wiesbaden: Gabler.

Polanyi, M., 1985: Implizites Wissen. Frankfurt.

Popitz, H./Bahrdt, H. P./Jüres, E. A./Kesting, H., 1957: Technik und Industriearbeit. Tübingen.

Porter, M. E., 1991: Nationale Wettbewerbsvorteile. Wien: Ueberreuter.

Projektgruppe Automation und Qualifikation, 1980: Automationsarbeit. Empirie 1, Argument Sonderband 43, Berlin

Rathschlag, A., 1972: Der Anlernberuf als Ausbildungsberuf für Jugendliche. Über die Entwicklung und Bedeutung einer berufspädagogisch umstrittenen Ausbildungskonzeption. Köln.

Reindl, J., 1998: Der Meister im Klein- und Mittelbetrieb: Von der Stabilität einer betrieblichen Sozialfigur., in: R. Jansen/O. Hecker/D. Scholz (Hrsg.): Facharbeiteraufstieg in der Sackgasse? Entwicklungen und Perspektiven auf der mittleren Qualifikationsebene. Bielefeld: W. Bertelsmann. S. 183-195

Riebel, P., 1955: Die Kuppelproduktion: Betriebs- und Marktprobleme. Köln: Westdeutscher Verlag.

Romanowski, G., 1994: Strukturelle Veränderungen und Standortschwächen der deutschen chemischen Industrie., S. 206-215 in: R. Simons/K. Westermann (Hrsg.): Wirtschaftsstandort Deutschland. Wettbewerbsfähigkeit. Zukunftschancen. Verteilungsspielräume. Köln: Bund-Verlag.

Roth, S., 1998: Das flexible Unternehmen.: Gabler.

Scharping, M., 2000: Vorwort, S. 11-23 in: ISF München (Hrsg.): Innovation und Arbeit. Berlin: Sigma.

Schmiede, R., o.J.: Branchenberichte: Die deutsche Chemische Industrie; unveröffentlichtes Manuskript.

Schudlich, E., 1982: Tarifpolitik ohne Kampfgeschrei in: U. Billerbeck (Hrsg.): Neuorientierung der Tarifpolitik? Veränderungen im Verhältnis zwischen Lohn- und Manteltarifpolitik in den siebziger Jahren. Frankfurt a.M. 
Schumann, M., 1998: Frißt die Shareholder-Value-Ökonomie die Modernisierung der Arbeit?, S. 19-30 in: H. Hirsch-Kreinsen/H. Wolf (Hrsg.): Arbeit, Gesellschaft, Kritik. Orientierungen wider den Zeitgeist. Berlin: Edition Sigma.

Schumann, M., 2003: Struktureller Wandel und Entwicklung der Qualifikationsanforderungen, S. 124-135 in: ders.: Metamorphosen von Industriearbeit und Arbeiterbewusstsein. Hamburg: VSA.

Schumann, M., 2003: Metamorphosen von Industriearbeit und Arbeiterbewusstsein. Kritische Industriesoziologie zwischen Taylorismusanalyse und Mitgestaltung innovativer Arbeitspolitik. Hamburg: VSA

Schumann, M./Baethge-Kinsky, V./Kuhlmann, M./Kurz, C./Neumann, U., 1994: Trendreport Rationalisierung. Automobilindustrie. Werkzeugmaschinenbau. Chemische Industrie. Berlin: Edition Sigma.

Schumann, M./Kuhlmann, M., 2000: Was bleibt von der Arbeitersolidarität? in: W. Dostal/P. Kupka (Hrsg.): Globalisierung, veränderte Arbeitsorganisation und Berufswahl. Beiträge zur Arbeitsmarkt- und Berufsforschung. Nürnberg: IAB.

Springer, R., 1998: Das Ende neuer Produktionskonzepte? Rationalisierung und Arbeitspolitik in der deutschen Automobilindustrie, S. 31-58 in: H. Hirsch-Kreinsen/H. Wolf (Hrsg.): Arbeit, Gesellschaft, Kritik. Orientierungen wider den Zeitgeist. Berlin: Edition Sigma.

Springer, R., 1999: Von der teilautonomen zur standardisierten Gruppenarbeit. Arbeitspolitische Perspektiven in der Automobilindustrie, in: WSI-Mitteilungen, 5, S. 309-321.

Staudt, E., (Hrsg), 1997: Kompetenz und Innovation. Eine Bestandsaufnahme jenseits von Personalentwicklung und Wissensmanagement. Innovation: Forschung und Management, Band 10. Bochum.

Stehr, N., 2001: Wissen und Wirtschaften. Die gesellschaftlichen Grundlagen der modernen Ökonomie. Frankfurt: Suhrkamp.

Streck, W. R., 1994: Zukunftschancen der chemischen Industrie, S. 193-205 in: R. Simons/K. Westermann (Hrsg.): Wirtschaftsstandort Deutschland. Wettbewerbsfähigkeit. Zukunftschancen. Verteilungsspielräume. Köln: Bund-Verlag.

Strube, J., 1995: Perspektiven des Standortes Deutschland für die chemische Industrie. Politische, gesellschaftliche und unternehmerische Voraussetzungen. in: J. Walter/U. Steger (Hrsg.): Fortschritt kommt nicht von alleine. Innovationsorientierte Strategien für eine intelligente Wirtschaftsentwicklung. Düsseldorf: Econ.

Tacke, V., 1997: Rationalitätsverlust im Organisationswandel. Von den Waschküchen der Farbenindustrie zur informatisierten Chemieindustrie,. Frankfurt a.M., New York: Campus Verlag.

Teltschik, W., 1992: Geschichte der deutschen Großchemie. Entwicklung und Einfluß in Staat und Gesellschaft. Weinheim, New York, Basel, Cambridge: VCH Verlagsgesellschaft.

Torstendahl, R., 1982: Engineers in industry, 1850-1910: Professional men and new bureaucrats. A comparative approach., S. 253-270 in: C. G. Bernhard/E. Crawford/P. Sörbom (Hrsg.): Science and Technology in the Time of Alfred Nobel. Oxford, New York: Pergamon Press. 
Tullius, K. (2003): Interne Kontraktualisierung und Meisterfunktion. Eine empirische Untersuchung neuer Formen betrieblicher Steuerung und der Funktion des Meisters in zwei Betrieben der deutschen Automobilindustrie. Dissertation. Göttingen. unveröffentlichtes Manuskript.

Turner, S., 1995: The Social Theory of Practices - Tradition, Tacit Knowledge and Presuppositions. Cambridge.

VCI: Chemiewirtschaft in Zahlen. diverse Jahrgänge.

Verg, E./Plumpe, G./Schultheis, H., 1988: Meilensteine. 125 Jahre Bayer. 1863-1988. Leverkusen.

Voß, G./Pongratz, H. J., 1998: Der Arbeitskraftunternehmer: eine neue Grundform der Ware Arbeitskraft?, in: KZfSS, 50, S. 131-158.

Weingart, P., 2001: Die Stunde der Wahrheit? Zum Verhältnis der Wissenschaft zu Politik, Wirtschaft und Medien in der Wissensgesellschaft. Weilerswist: Velbrück Wissenschaft.

Wetzel, W., 1991: Naturwissenschaften und Chemische Industrie in Deutschland. Voraussetzungen und Mechanismen ihres Aufstiegs im 19. Jahrhundert. Stuttgart: Franz Steiner Verlag.

Wetzel, W., 1998: Origins of education and career opportunities for the profession of 'chemist' in the second half of the nineteenth century in Germany., S. 77-94 in: D. Knight/H. Kragh (Hrsg.): The Making of the Chemist. The Social History of Chemistry in Europe, 1789-1914. Cambridge: Cambridge University Press.

Wiendahl, H.-P., 1997: Betriebsorganisation für Ingenieure. München/Wien: Carl Hanser. Willke, H., 1999: Systemisches Wissensmanagement. Stuttgart: Lucius\&Lucius.UTB.

Wittel, A., 1998: Gruppenarbeit und Arbeitshabitus, in: Zeitschrift für Soziologie, 27 (3), S. $178-192$.

Wittke, V., 1990: Systemische Rationalisierung - Zur Analyse aktueller Umbruchprozesse in der industriellen Produktion., S. 23-42 in: J. Bergstermann/R. Brandherm-Böhmker (Hrsg.): Systemische Rationalisierung als sozialer Prozeß. Bonn: J.H.W. Dietz Verlag.

Wittke, V., 1995: Vertikale versus horizontale Desintegration - Zu unterschiedlichen Erosionsdynamiken des Großunternehmens im Prozeß industrieller Restrukturierung., in: SOFI-Mitteilungen, 22, S. 7-15.

Wittke, V., 1996: Wie entstand industrielle Massenproduktion? Die diskontinuierliche Entwicklung der deutschen Elektroindustrie von den Anfängen der 'großen Industrie' bis zur Entfaltung des Fordismus (1880-1975). Berlin: Edition Sigma.

Wittke, V./Buss, K.-P., 2001: Wissen als Ware - Überlegungen zum Wandel der Modi gesellschaftlicher Wissensproduktion am Beispiel der Biotechnologie in: G. Bender (Hrsg.): Neue Formen der Wissenserzeugung. Frankfurt, New York: Campus.

Wöcherl, H., 1989: Die Modernisierung der Chemischen Industrie und deren arbeitspolitische Konsequenzen, S. 199-253 in: L. Pries/R. Trinczek/R. Schmidt (Hrsg.): Trends betrieblicher Produktionsmodernisierung. Opladen: Westdeutscher Verlag.

Wolf, H., 1999: Arbeit und Autonomie. Münster. Westfälisches Dampfboot.

Wolf, H., 1994: Rationalisierung und Partizipation, in: Leviathan, 2, S. 243-259. 
Yan, A./Louis, M. R., 1999: The Migration of Organizational Functions to the Work Unit Level: Buffering, Spanning, and Bringing Up Boundaries., in: Human Relations, 52 (1), S. 25-47.

Zeller, C., 2001: Globalisierungsstrategien - Der Weg von Novartis. Berlin, Heidelberg: Springer. 


\section{Curriculum Vitae}

Name: $\quad$ Kendra Briken

Dipl- Sozialwirtin

geb. am: $\quad$ 18.05.1972

in: $\quad$ Alfeld / Leine

\section{Schulischer Werdegang}

$1978-1991 \quad$ Grundschule (Eime), Orientierungsstufe (Gronau) und Gymnasium (Alfeld), Abiturnote 1,6

\section{Wissenschaftlicher Bildungsgang}

WS 1991/992

SS $1992-$ SS 1994

WS 1994/95

SS 1995 - WS 1996/97
Studium der Rechtswissenschaften an der Universität Trier

Studium der Sozialwissenschaften an der Georg-AugustUniversität Göttingen

Studienaufenthalt am Institut d'Etudes Politiques in Paris (DAAD-Stipendium)

Studium der Sozialwissenschaften an der Georg-AugustUniversität Göttingen

Abschluss: Diplom Sozialwirtin, Note 2,0

Diplomarbeit bei Prof. Bassam Tibi, zum Thema „Deutschland und Frankreich: Kulturnation und Staatsnation im Vergleich.“ (Note 1,7)

wissenschaftliche Mitarbeiterin am Soziologischen Forschungsinstitut e.V. (SOFI) in Göttingen 
Ich versichere, dass ich die vorliegende Dissertation „Perspektiven von Arbeit in der Chemischen Industrie“ selbständig und ohne unerlaubte Hilfsmittel verfasst habe. Anderer als der von mir angegebenen Hilfsmittel und Schriften habe ich mich nicht bedient. Alle wörtlich oder sinngemäß den Schriften anderer Autoren und Autorinnen entnommenen Stellen habe ich kenntlich gemacht.

Kendra Briken, im September 2004 